\title{
A Cysteine Selenosulfide Redox Switch for Protein Chemical Synthesis
}

Vincent Diemer, Nathalie Ollivier, Bérénice Leclercq, Hervé Drobecq, Jérôme Vicogne, Vangelis Agouridas, Oleg Melnyk

Submitted date: 26/02/2020 Posted date: 26/02/2020

Licence: CC BY-NC-ND 4.0

Citation information: Diemer, Vincent; Ollivier, Nathalie; Leclercq, Bérénice; Drobecq, Hervé; Vicogne, Jérôme; Agouridas, Vangelis; et al. (2019): A Cysteine Selenosulfide Redox Switch for Protein Chemical Synthesis. ChemRxiv. Preprint. https://doi.org/10.26434/chemrxiv.11110133.v2

The control of cysteine reactivity is of paramount importance for the synthesis of proteins using the native chemical ligation (NCL) reaction. We discovered that this goal can be achieved in a traceless manner during ligation by appending a simple $\mathrm{N}$-selenoethyl group to cysteine. While in synthetic organic chemistry the cleavage of carbon-nitrogen bonds is notoriously difficult, we found that $\mathrm{N}$-selenoethyl cysteine (SetCys) loses its selenoethyl arm in water under mild conditions upon reduction of its selenosulfide bond. Detailed mechanistic investigations uncover a novel mode of reactivity for Cys. Its implementation in a process enabling the modular and straightforward assembly of linear or backbone cyclized polypeptides is illustrated by the synthesis of biologically active cyclic hepatocyte growth factor variants.

File list (2)

Article_R1_3.pdf (2.12 MiB) view on ChemRxiv - download file 


\title{
A cysteine selenosulfide redox switch for protein chemical synthesis
}

\author{
Vincent Diemer, Nathalie Ollivier, Bérénice Leclercq, Hervé Drobecq, Jérôme Vicogne, \\ Vangelis Agouridas*, Oleg Melnyk*
}

University of Lille, CNRS, Institut Pasteur de Lille, UMR CNRS 8204, INSERM U1019, Centre d'Immunité et d'Infection de Lille, F-59000 Lille, France

\begin{abstract}
The control of cysteine reactivity is of paramount importance for the synthesis of proteins using the native chemical ligation (NCL) reaction. We discovered that this goal can be achieved in a traceless manner during ligation by appending a simple $N$-selenoethyl group to cysteine. While in synthetic organic chemistry the cleavage of carbon-nitrogen bonds is notoriously difficult, we found that $\mathrm{N}$ selenoethyl cysteine (SetCys) loses its selenoethyl arm in water under mild conditions upon reduction of its selenosulfide bond. Detailed mechanistic investigations uncover a novel mode of reactivity for Cys. Its implementation in a process enabling the modular and straightforward assembly of linear or backbone cyclized polypeptides is illustrated by the synthesis of biologically active cyclic hepatocyte growth factor variants.
\end{abstract}

\section{Introduction}

In recent years, the study of protein function has made tremendous advances thanks to the development of chemical synthetic tools and strategies for producing peptides and proteins. The vast majority of proteins obtained this way are assembled using native chemical ligation ( $\mathrm{NCL}^{1}$, Figure 1a) or derived methods.$^{2,3,4,5} \mathrm{NCL}$ involves the reaction of a peptide thioester with a Cys peptide to produce a native peptide bond to Cys. The synthesis of complex protein scaffolds requires the control at some point of the reactivity of Cys for orienting the order by which the peptide bonds connecting the various peptide segments are produced (Figure 1a). ${ }^{6}$ Therefore, designing new strategies for modulating Cys 
reactivity is a contemporary concern and stimulates the creativity of protein and organic chemists worldwide. $^{7,8,9,10,11}$

One hallmark of the Cys residue is its involvement in the formation of disulfide or selenosulfide bonds (Figure 1b), ${ }^{12}$ which often play a critical role in protein folding. Nature also exploits the redox properties of Cys thiols to control the activity of some enzymes featuring a Cys residue at their catalytic site. ${ }^{13}$ Indeed, the conversion of a catalytic Cys thiol into a disulfide is a powerful means for shutting down enzymatic activity because disulfides are poor nucleophiles compared to thiolates. Thioredoxinreductase or glutathione reductase are typical examples where the enzymes become active upon reduction of a disulfide bond. ${ }^{13}$ In synthetic organic chemistry, the redox properties of the thiol group also offer a simple means for controlling its reactivity. ${ }^{14}$ Unfortunately, acyclic dichalcogenide derivatives of Cys are labile or in fast exchange under the reducing conditions used for performing NCL. Consequently, such a bioinspired control of NCL by using Cys thiol as a redox switch has not so far proved achievable. In practice, Cys reactivity is instead masked during protein assembly by introducing classical alkyl- or acyl-based protecting groups on the $\alpha$-amino group, on the side-chain thiol or both (for a recent review see reference ${ }^{2}$ ).

To circumvent the high lability of Cys acyclic disulfides during NCL and to use Cys thiol as a redox switch for controlling protein assembly, we sought to embed the Cys thiol in a cyclic dichalcogenide as such species are known to be significantly more oxidizing than their linear counterparts. ${ }^{15}$ In this work, we explored the properties of SetCys, the cyclic selenosulfide obtained by introducing a selenoethyl appendage on the $\alpha$-amino group of Cys (Figure 1c). We discovered that the products of NCL with SetCys peptides vary with the strength of the reducing agent. Importantly, SetCys spontaneously loses its selenoethyl arm in water at neutral $\mathrm{pH}$ in the presence of popular disulfide bond reductants such as dithiothreitol (DTT) or tris(2-carboxyethyl)phosphine (TCEP). This chemical behavior contrasts with the known difficulty in breaking carbon-nitrogen bonds, a process that usually requires harsch conditions, ${ }^{16,17}$ metal catalysis ${ }^{18}$ or radical reactions. ${ }^{19,}{ }^{20}$ In contrast, the detailed mechanistic investigations reported here point toward an anionic mechanism that depends on the ionization state of SetCys in its ring-opened and reduced form. In this respect, SetCys uncovers a novel mode of reactivity for Cys and provides a useful means for accessing complex protein scaffolds as illustrated by the total 
one-pot synthesis of biologically active linear or backbone cyclized variants of the hepatocyte growth factor (HGF) kringle 1 (K1) domain.

\section{Results}

\section{SetCys peptides display an array of reactivities depending on the reducing environment}

The NCL reaction is classically performed in the presence of aryl thiol catalyts, ${ }^{21}$ of which 4mercaptophenylacetic acid (MPAA) is considered as the gold standard. ${ }^{22}$ In addition to its catalytic abilities, the latter also contributes to the maintenance of the reactants in a weakly reducing environment. MPAA can possibly be complemented by DTT and TCEP, two powerful reductants that are popular additives for NCL. Thus, MPAA and MPAA/DTT or MPAA/TCEP additive cocktails define two extremes in reductive power applied to ligation mixtures.

We first examined the behavior of the SetCys residue in the presence of MPAA alone, i.e., weakly reductive conditions, in the search for conditions where it could be silent. Exposure of a model SetCys peptide to a large excess of MPAA at neutral $\mathrm{pH}$ led to no apparent change (Figure 2a, property 1). In a second experiment, incubation of the SetCys peptide with a peptide thioester in the presence of MPAA furnished a ligated peptide featuring an internal SetCys residue (Figure 2a, property 2). Although we could not detect any reduced SetCys in the presence of MPAA alone (Figure 2a, property 1), perhaps due to its oxidation by molecular oxygen during workup and analysis, the formation of the SetCys amide product in this experiment shows that this species is likely present under these conditions. However, the rate of ligation was more than 10 fold lower than the rate observed for NCL with a Cys peptide. This observation prompted us to run a competitive reaction in which a peptide thioester and an equimolar mixture of SetCys and Cys peptides were reacted in the presence of MPAA (Figure 2a, property 3). Interestingly, this experiment resulted in the exclusive formation of the ligation product with the Cys peptide. We further showed that the SetCys peptide does not interfere with NCL even when the thioester component features a sterically demanding amino acid at its C-terminus, typically a valine residue (see Supporting Information). We also verified that internal Cys residues are unable to activate SetCys residue, which is therefore useful for the production of Cys-rich peptides (see Supporting Information). 
Thus, the background NCL observed for a SetCys peptide in the presence of MPAA is unable to perturb a regular NCL involving a Cys peptide.

The most striking property of SetCys was observed when the SetCys peptide was subjected to the strong reducing conditions imposed by DTT or TCEP (Figure 2a, reaction 4). In this case, the reaction cleanly furnished the Cys peptide. We further documented that the reaction of a SetCys peptide with a peptide thioester in the presence of TCEP furnished a ligation product featuring a native Cys residue at the ligation junction (Figure 2a, reaction 5). In contrast, the loss of the $N$-alkyl substituent was not observed when the sulfur analog of SetCys, featuring a 2-mercaptoethyl group on the $\alpha$-nitrogen, was treated similarly, even after extended reaction times (Figure 2b). ${ }^{23,}{ }^{24}$ The reactivity observed for SetCys depends specifically on the presence of selenium in its structure and, in that respect, SetCys is a novel illustration of the high difference in reactivity than can exist between thiol and selenol compounds. ${ }^{25}$

\section{Insights into the conversion of SetCys to a Cys residue}

From a mechanistic point of view, the loss of the selenoethyl group from the SetCys residue seems unlikely to involve radical intermediates since the reaction proceeds well in the presence of a large excess of sodium ascorbate and MPAA, ${ }^{26,27}$ two reagents known to be powerful quenchers of alkylselenyl or alkylthiyl radicals. Omitting ascorbate during the treatment of SetCys peptide $\mathbf{1}$ by TCEP yields the deselenized peptide Et-CALKEPVHGV-NH 2 as the major product, whose formation competes against the loss of the selenoethyl arm (see Supporting Information). Furthermore, the loss of the selenoethyl limb is also observed when dithiothreitol is used as a reducing agent, definitely ruling out the possibility that the reaction might involve a classical TCEP-induced dechalcogenation process. ${ }^{28,}$

${ }^{29}$ Further insights into the species involved in the reaction come from the data shown in Figure 3b, which presents the effect of $\mathrm{pH}$ on the rate of selenoethyl limb removal from a model SetCys peptide 2. The $\mathrm{pH}$-rate profile of the conversion of SetCys peptide $\mathbf{2}$ into cysteinyl peptide $\mathbf{3}$ shows a maximum at $\mathrm{pH} 6.0 \pm 0.04$ and two inflexion points at $\mathrm{pH} 4.8$ and 7.3, which likely correspond to the $\mathrm{pK}_{a} \mathrm{~s}$ of the SetCys selenol and ammonium groups respectively. These values are in agreement with the $\mathrm{pK}_{a}$ values reported for simple 2-selanylethylamines ${ }^{30}$ and Cys derivatives ${ }^{31}$ or estimated by calculation (Figure 3c). The fact that the $\mathrm{pH}$-rate profile of the reaction corresponds to the predominance zone for the 
selenoate/ammonium zwitterionic intermediate $\mathbf{2}^{+-}$led us to propose that the decomposition of SetCys proceeds through the intramolecular substitution of the ammonium group by the selenide ion.

This mechanism results in the formation of an episelenide, which is known to be extremely unstable at room temperature and spontaneously decomposes into ethylene and selenium (Figure 3a). ${ }^{17}$ While selenium can be captured by TCEP in the form of the corresponding selenophosphine, whose formation was indeed observed in these reactions, detection of ethylene gas was made difficult by the small scale of synthesis.

The proposed mechanism is reminiscent of the cleavage of alkylamines by phenylselenol, albeit such reactions usually require elevated temperatures and/or assistance by metals. ${ }^{16,17,32}$ Intrigued by the ease of SetCys to Cys conversion, we sought to determine if the SetCys thiol participates to the departure of the 2-selanylethyl limb. To this end, a $N$-(2-selanylethyl)-alanyl (SetAla) peptide analogue was prepared and treated with MPAA/TCEP/ascorbate at the optimal $\mathrm{pH}$ for the SetCys to Cys conversion, i.e., $\mathrm{pH}$ 6.0 (Figure 3d). LC-MS analysis of the mixture showed the conversion of the SetAla residue into Ala, but at a rate considerably lower ( $~ 8.5$ fold) than those measured for the SetCys to Cys conversion. This experiment shows that the departure of the 2-selanylethyl limb is greatly facilitated by the nearby SetCys thiol, perhaps by allowing an intramolecular proton transfer as depicted in Figure 3a.

\section{Insights into the mechanism of SetCys-mediated ligation}

Having scrutinized the mechanism of SetCys conversion to a Cys residue under strong reductive conditions, we next examined the species involved during ligation with a peptide alkyl thioester under the same redox conditions. The monitoring of the reaction between SetCys peptide $\mathbf{1}$ and peptide thioester $\mathbf{4}$ indicated that a first ligation product $\mathbf{5}$, containing an internal SetCys residue, accumulated within the first minutes and then slowly disappeared over two days in favor of peptide $\mathbf{6}$ featuring a native peptide bond to Cys (Figure 4a,b).

Regarding the mechanism of SetCys-mediated ligation under strong reducing conditions, we hypothesized that the early formation of intermediate $\mathbf{5}$ is due to the interception of the reduced SetCys unit $\mathbf{2}$ by the thioester component. The latter is likely to be present in the form of the aryl thioester $\mathbf{7}$, produced in situ from peptide alkyl thioester $\mathbf{4}$ by thiol-thioester exchange with the MPAA catalyst 
(Figure 4c). Of the two nucleophilic sites present in reduced SetCys unit, the selenol moiety is probably the more reactive due to its lower $\mathrm{pK}_{a}$ and higher nucleophilicity. The formation of tertiary amides of type $\mathbf{5}$ is known to be reversible in the conditions used for the ligation through their capacity to undergo an intramolecular nitrogen to selenium or sulfur acyl group migration. ${ }^{23,24,33}$ Therefore, SetCys peptide 2 is constantly present in solution and escapes the SetCys/SetCys amide equilibrium by irreversibly losing its $N$-selenoethyl limb as discussed above. The Cys peptide $\mathbf{3}$ produced this way is expected to undergo a classical NCL reaction with aryl thioester 7 to yield ligated Cys peptide 6 . Although the proposed mechanism arises from the properties of the SetCys unit described in Figure 2, we sought to confront it to kinetic data for validation. In addition, the model also tests the possibility of a direct conversion of SetCys amide 5 into final product $\mathbf{6}$, being fully aware that the cleavage of the selenoethyl appendage from compound $\mathbf{5}$ through an ionic mechanism is unlikely due to the poor leaving group ability of imido nitrogens.

We first determined the rate constants associated with the thiol-thioester exchange process involving peptide thioester $\mathbf{4}$ and MPAA $\left(\mathrm{k}_{+2}, \mathrm{k}_{-2}\right)$, with the conversion of SetCys peptide $\mathbf{2}$ into Cys peptide $\mathbf{3}\left(\mathrm{k}_{1}\right)$ and with the reaction of peptide aryl thioester 7 with Cys peptide $3\left(\mathrm{k}_{4}\right)$, from model reactions run separately (see Supplementary Information). These rate constants were used to determine the remaining kinetic parameters $\mathrm{k}_{+3}, \mathrm{k}_{-3}$ and $\mathrm{k}_{5}$ by fitting the experimental data of the SetCys-mediated ligation (circles and triangles in Figure 4b) to the mechanistic model. The quality of the fit (dashed lines in Figure 4b, $p$ $=0.003$ ) strongly suggests that the conversion of intermediate 5 to peptide 6 exclusively occurs through SetCys peptide $\mathbf{2}$ since the kinetic constant for the direct process $\mathbf{5} \rightarrow \mathbf{6}$ is at least 100 fold below the value measured for the decomposition of the SetCys into the Cys residue, i.e. $\mathbf{2} \rightarrow \mathbf{3}$. The second piece of information provided by the model is that the SetCys peptide in its reduced form reacts almost as fast with the peptide thioester component as does the Cys peptide. Finally, the model tells us that the loss of the $N$-selenoethyl sidechain from SetCys peptide $\mathbf{2}$ is the rate limiting step of SetCys-mediated ligation.

\section{SetCys redox-switch enables the straightforward synthesis of cyclic proteins in one-pot}

Having characterized the differential reactivity of the SetCys unit under mild and strong reducing conditions, we further sought to develop a simple process enabling the synthesis of cyclic 
proteins using SetCys as a redox switch (Figure 5). The motivation for this particular application comes from the observation that although a few studies pointed out the potential of protein cyclization for improving protein thermal stability, ${ }^{34,}{ }^{35}$ resistance to proteolytic degradation ${ }^{35}$ and potency,${ }^{36}$ this modification has not so far been widely used for the design of protein therapeutics. This situation contrasts with the success of small cyclic protein scaffolds such as cyclotides used as platforms for drug design, ${ }^{37}$ and the frequent use of macrocyclization in the development of small peptidic drugs. ${ }^{38}$ The fact that protein cyclization is seldom used for protein optimization cannot be ascribed to inappropriate $\mathrm{N}-\mathrm{C}$ distances, because half of the protein domains found in the protein data bank (PDB) have their C and $\mathrm{N}$ extremities joinable by linkers made of a few amino acids. ${ }^{39}$ Rather, this situation reflects the paucity of tools for building cyclic proteins in a modular approach that facilitates the optimization of the linker joining the N- and C-termini. ${ }^{40}$

Classical methods leading to cyclic proteins involve the macrocyclization of a bifunctional and linear precursor ${ }^{41}$ primarily by using the native chemical ligation reaction $\left(\mathrm{NCL}^{1}\right)$ between a $\mathrm{C}$-terminal thioester group and an N-terminal cysteine (Cys) residue (Figure 5a). ${ }^{2}{ }^{42}$ Following this strategy, the optimization of the linker requires the production of a library of extended precursors of varying length and composition, an approach that inevitably makes the production of cyclic analogs cumbersome.

In this work, we sought to develop a modular one-pot method enabling the grafting of the linker to a unique linear protein precursor (Figure 5b). This can be achieved by exploiting the silent properties of the SetCys residue under mild reducing conditions for performing the first NCL (see property 3, Figure 2a), and then by using it as a redox switch for triggering the second cyclative NCL (see property 5 , Figure 2a). Regarding the acyl donors, the process utilizes the good reactivity of alkyl thioesters in the presence of MPAA for the first ligation step. The second acyl donor is introduced as the bis(2sulfenylethyl)amido (SEA) ${ }^{43}$ latent thioester surrogate, which nicely complements the SetCys unit since it can also be activated in the presence of TCEP. ${ }^{44,45}$ The Cys / SEA couple of functional groups is located on the linear protein precursor, while the SetCys / alkyl thioester functionalities are on the linker peptide. In the presence of MPAA alone, the Cys-mediated NCL with the peptide alkyl thioester (Step 1, Figure 5b) exclusively yields a bifunctional polypeptide intermediate, which is activated at both ends by the addition of TCEP in one-pot (Step 2) to produce the backbone-cyclized polypeptide (Step 3). The 
process is highly tolerant of polypeptide length as similar isolated yields were obtained for the production of medium to large cyclic peptides (30-93 AAs, $\sim 27-37 \%$ overall yields). The examples include the synthesis of two cyclic and biotinylated variants of hepatocyte growth factor / scatter factor (HGF/SF) kringle 1 domain (K1), i.e., cK1-1 and cK1-2, from a unique 78 AA linear precursor. These cyclic polypeptides differ by the length of the linker joining $\mathrm{N}$ and C-termini of the K1 protein (10 and 14 residues respectively).

SetCys chemistry proved equally useful for the C-to-N one-pot assembly of linear polypeptides from three peptide segments (Figure 5a,c). The peptide segments could be added sequentially (Figure 5c, Method A) or mixed altogether from the beginning of the assembly process (Figure 5c, Method B) with equal selectivity and efficiency.

\section{Folding and biological activity of biotinylated K1 cyclic analogs}

The signaling of the HGF/SF/MET system plays a crucial role in the regeneration of several tissues such as the liver or the skin, while its deregulation is often observed in cancer. The HGF/SF K1 domain contains the main HGF/SF binding site for the MET tyrosine kinase receptor and thus constitutes an interesting platform for designing future drugs based on this couple of proteins. ${ }^{46}$ In this study, we sought to produce cyclic analogues of the $\mathrm{K} 1$ domain to investigate the tolerance of the K1/MET signaling system to this modification. The X-ray crystal structure of the K1 protein shows that its tertiary structure is made up of a series of loops stabilized by three disulfide bonds (Figure 6a). ${ }^{47}$ The N- and C-terminal cysteine residues are on the opposite side of the MET binding loop and are linked by a disulfide bond. The $\mathrm{N}$ - and $\mathrm{C}$ termini are thus close in space and can be joined by a peptide linker made of a few amino acid residues which include a biotinylated lysine residue. The latter is used to multimerize the ligand using streptavidin (S) due to the observation that multivalent presentation of the K1 domain is important for achieving high binding and agonistic activities. ${ }^{46}$

The successful synthesis of the cyclic K1 polypeptides cK1-1 and cK1-2 set the stage for the folding step. cK1-1 and cK1-2 were folded into cK1-1f and cK1-2f respectively using the glutathione/glutathione disulfide redox system (Figure 6b,c). ${ }^{45}$ The folding mixtures converged to a major form after $24 \mathrm{~h}$ and were purified by dialysis (Figure 6c, see also Supplementary Information). 
Extensive proteomic analysis of the folded proteins cK1-1f and cK1-2f showed the exclusive formation of the native pattern of disulfide bonds between Cys128-206, Cys149-189 and Cys177-201 as shown in Figure $6 \mathrm{~b}$ (see Supplementary Information). Thus the cyclization does not perturb the correct pairing of the Cys residues.

cK1-1f and cK1-2f proteins were first analyzed for their capacity to bind to the recombinant MET extracellular domain. The competitive AlphaScreen ${ }^{\circledR}$ assay with recombinant NK1 protein showed that the backbone cyclized proteins cK1-1f and cK1-2f were $\sim 10$ fold less potent in binding the MET receptor than the biotinylated analog K1B (Figure 6d). This result was unexpected because the cyclization site is diametrically opposite the MET binding site. The capacity of the cyclic K1 proteins to activate the MET receptor was further examined using human HeLa cells (Figure 6e). MET phosphorylation induced by the cyclic K1 proteins was found to be less than that observed with the reference K1B analog. However, the tested K1 analogs triggered downstream signaling pathways, i.e., phosphorylation of AKT and ERK, with almost equal potency. Because previous studies showed marked differences between MET phosphorylation levels and the strength of MET specific phenotypes induced by HGF or HGF mimics, ${ }^{48}$ we further analyzed the capacity of the different K1 proteins to trigger the scattering of human cells in vitro (Figure 6f). In this assay using human Capan 1 cells, the cyclic proteins behaved similarly to the reference protein K1B in the concentration range tested (10 pM-100 nM) by their capacity to induce a mesenchymal-like phenotype and cell scattering. To summarize, cyclization in this case results in a significant loss of affinity, although the backbone cyclization site is opposite to the receptor binding site. However, this loss of affinity does not translate into the cell scattering activity. This work highlights the need for simple synthetic methods toward cyclic proteins to rapidly investigate the interest of backbone cyclization for improving protein properties.

In conclusion, the cyclic selenosulfide derivative of cysteine, i.e. SetCys, shows an array of reactivities in water depending on the reducing power of the solution. A striking property of SetCys is its conversion to a native Cys residue by cleavage of a nitrogen-carbon bond under extremely mild conditions if a strong reducing agent is present in the solution. This transformation also occurs in the presence of a peptide thioester component and thus leads to the production of a native peptide bond to Cys by native chemical ligation. In contrast, SetCys remains silent during NCL if it is conducted under 
mild reducing conditions. The redox-switch properties of SetCys are particularly adapted for flexible synthetic designs that involve nothing more than common additive that are routinely used in NCL reaction.

\section{Acknowledgements}

We thank ANR for financial support (CyProt, ANR-19CE07-0020).

\section{Author information}

\section{Affiliations}

University of Lille, CNRS, Institut Pasteur de Lille, UMR CNRS 8204, INSERM U1019

Vincent Diemer, Nathalie Ollivier, Bérénice Leclercq, Hervé Drobecq, Jérôme Vicogne, Vangelis Agouridas, Oleg Melnyk

\section{Author contributions}

VD performed the experiments and wrote the manuscript. NO prepared the linear K1 precursor. HD performed the proteomic experiments. BL and JV performed the AlphaScreen ${ }^{\circledR}$ and the cell-based assay. VA performed the modelization study and wrote the manuscript. OM conceived the study and wrote the manuscript.

\section{Corresponding authors}

Correspondence to Oleg Melnyk or Vangelis Agouridas.

\section{Ethics declarations}

\section{Competing interests}

The authors declare no competing interests.

\section{Supplementary information}


Experimental details, materials and methods, kinetic model for SetCys-mediated ligation, LC-MS data and NMR spectra.

\section{Data Availability}

The source data underlying Figs 6d-f are provided as a Source Data file. 
a

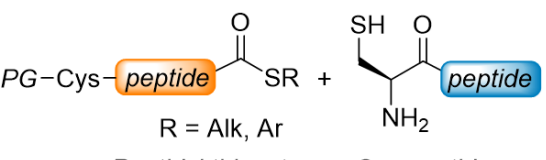

Peptidyl thioester Cys peptide

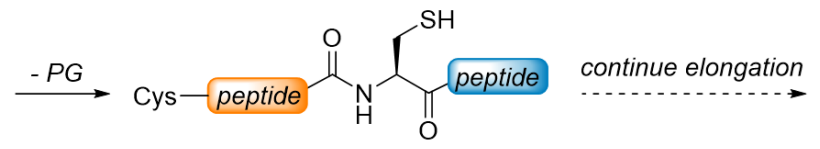

Ligated Cys peptide

b

e.g., distant Cys/Sec residues, Glutathione

Cys disulfide $(X=S)$ or selenosulfide $(X=S e)$

Poor nucleophilicity Silent

(1)

Cys thiolate

High nucleophilicity

Reactive

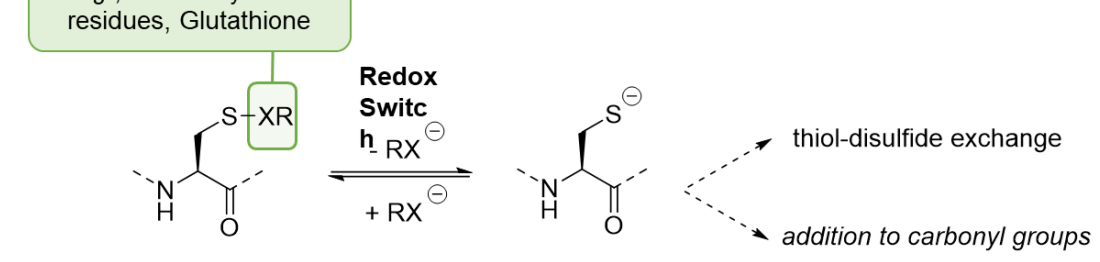

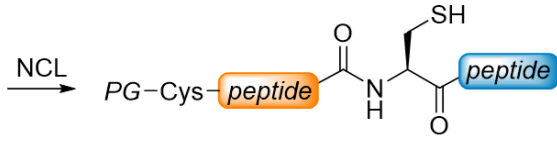

Ligated Cys peptide

C This work: Traceless control of cysteine reactivity by a cyclic selenosulfide

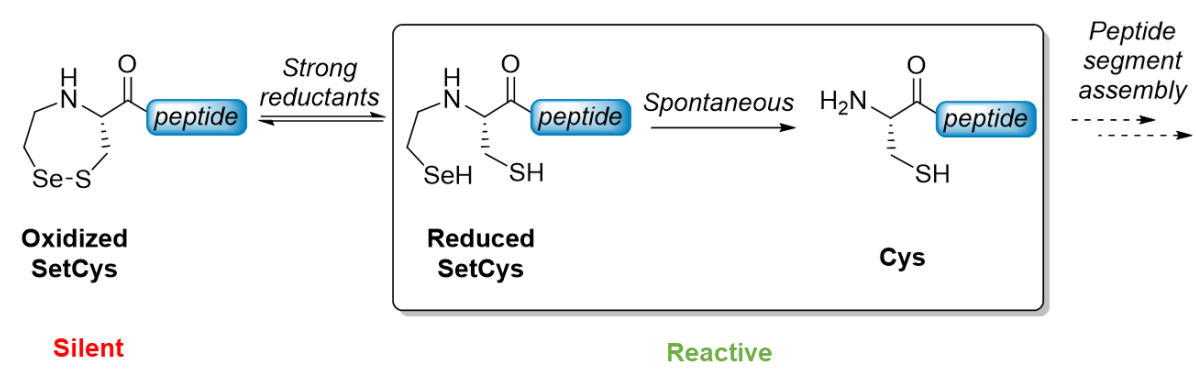

Figure 1. a) Principle of the native chemical ligation (NCL) between a C-terminal peptide thioester and a cysteinyl (Cys) peptide. The control of the site of ligation requires masking any $\mathrm{N}$-terminal Cys residue present in the mixture. b) The reversible formation of disulfide or diselenide bonds is a hallmark of Cys thiol chemistry in proteins and is used by nature as a redox switch to control Cys thiol reactivity. c) The $N$-(2-selanylethyl) group of SetCys shuts down the nucleophilic properties of Cys thiol by formation of a cyclic selenosulfide bond. It is removed spontaneously in water at neutral $\mathrm{pH}$ under reductive conditions. 


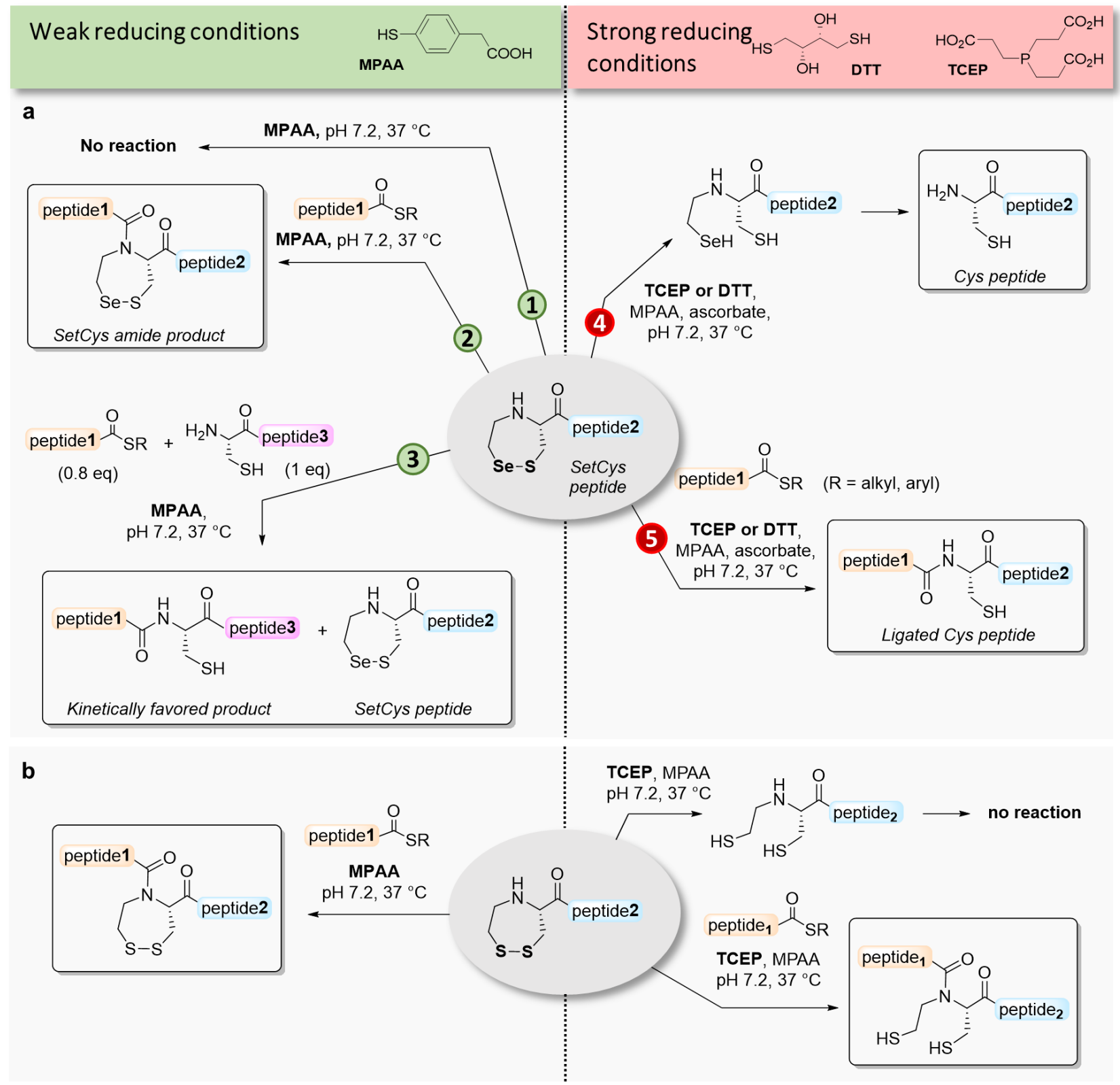

Figure 2. a) The reactivity of $N$-(2-selanylethyl)cysteine (SetCys) peptides is controlled by the reducing strength of the mixture. The numbers on the arrows indicate different experimental conditions. Condition 1-3: weakly reducing conditions, typically in the presence of an excess of 4mercaptophenylacetic acid (MPAA) at neutral $\mathrm{pH}$. Conditions 4 and 5: strong reducing conditions, typically in the presence of dithiothreitol (DTT) or tris(2-carboxyethyl)phosphine (TCEP) at neutral pH. b) Ligation of $N$-(2-sulfanylethyl)cysteine (SutCys) peptides with peptide alkyl thioester under weak or strong reducing conditions. Peptide sequences: peptide $1=$ RLKEPVHGA-, peptide $2=$ ALKEPVHGV$\mathrm{NH}_{2}$, peptide $3=$ ILKEPVHGV-NH${ }_{2}$. 

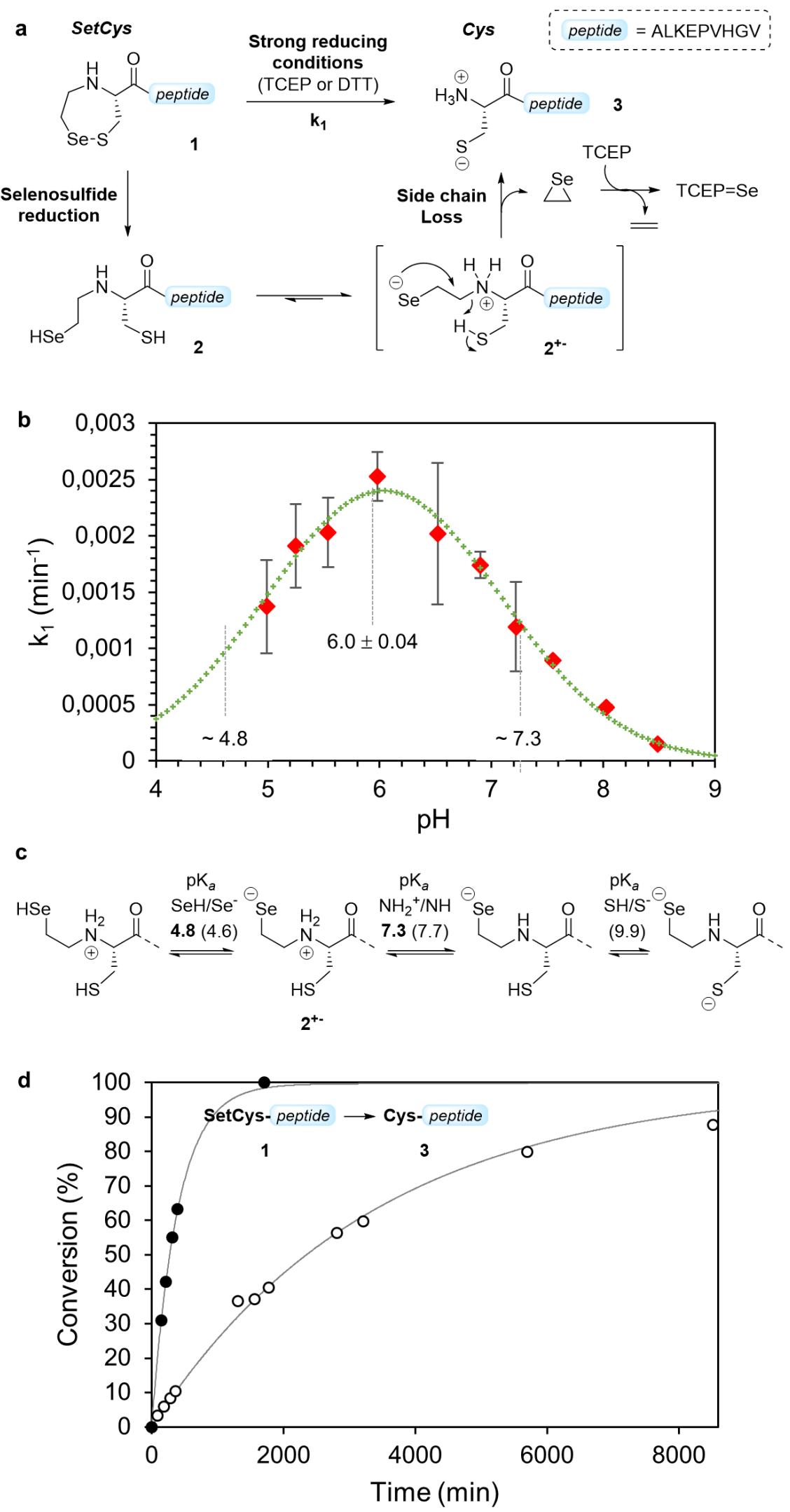

Figure 3. a) Proposed mechanism for the loss of the $N$-selenoethyl appendage of $\mathrm{N}$-terminal SetCys peptides; b) Experimental $\mathrm{pH}$-rate profile of the conversion of N-terminal SetCys peptides to the 
corresponding N-terminal cysteinyl peptide (red diamonds). The data were fitted to a Gaussian (green curve, $p<0.001$ ) to determine the $\mathrm{pH}$ values for the inflexion points ( $\mathrm{pH} 4.8$ and 7.3). c) Different ionization states for the SetCys residue in open form. The $\mathrm{pK}_{a}$ values in bold correspond to the inflexion points determined in $b$. The values in parentheses were calculated using ACDLabs ${ }^{\circledR}$ software. d) Rate of 2-selanylethyl limb cleavage in SetCys $\left(\bullet, \mathrm{k}=2.53 \times 10^{-3} \mathrm{~min}^{-1}\right)$ and SetAla $\left(\mathbf{O}, \mathrm{k}=2.96 \times 10^{-4} \mathrm{~min}^{-}\right.$ $\left.{ }^{1}\right)$ model peptides at $\mathrm{pH} 6$. 
a

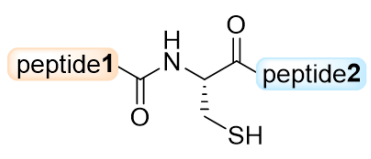

6 (thermodynamic product) $64 \%$ isolated yield
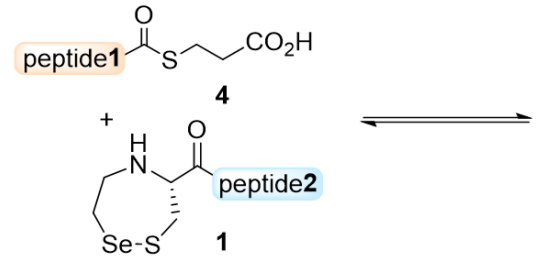

5 (kinetic product)

Experimental conditions for the kinetic study: $0.1 \mathrm{M}$ phosphate buffer, $6 \mathrm{M} \mathrm{Gn} \mathrm{HCl,} 200 \mathrm{mM}$ MPAA, $100 \mathrm{mM}$ TCEP, $100 \mathrm{mM}$ sodium ascorbate, $\mathrm{pH} 7.2,37^{\circ} \mathrm{C}$. 1: $9.6 \mathrm{mM}, 4: 8 \mathrm{mM}$
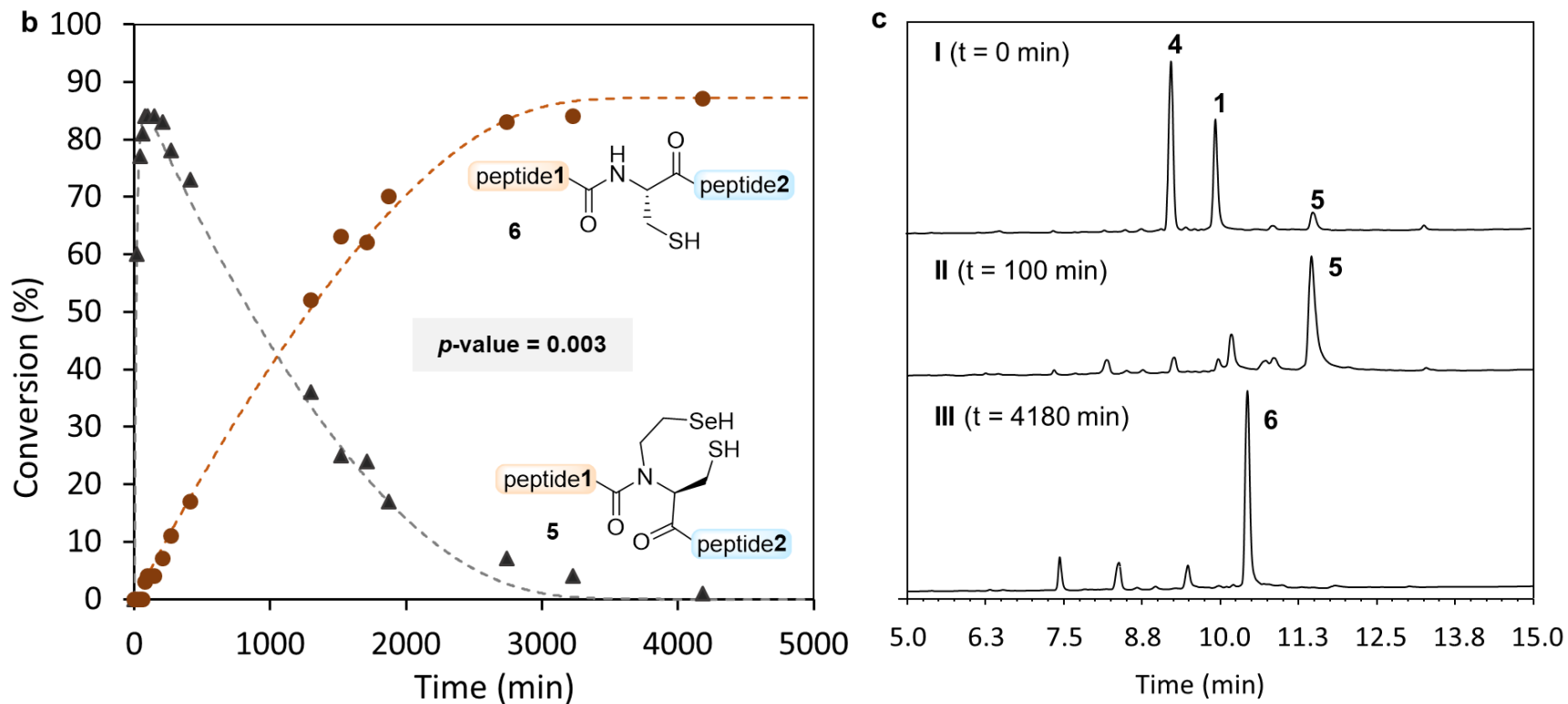

d

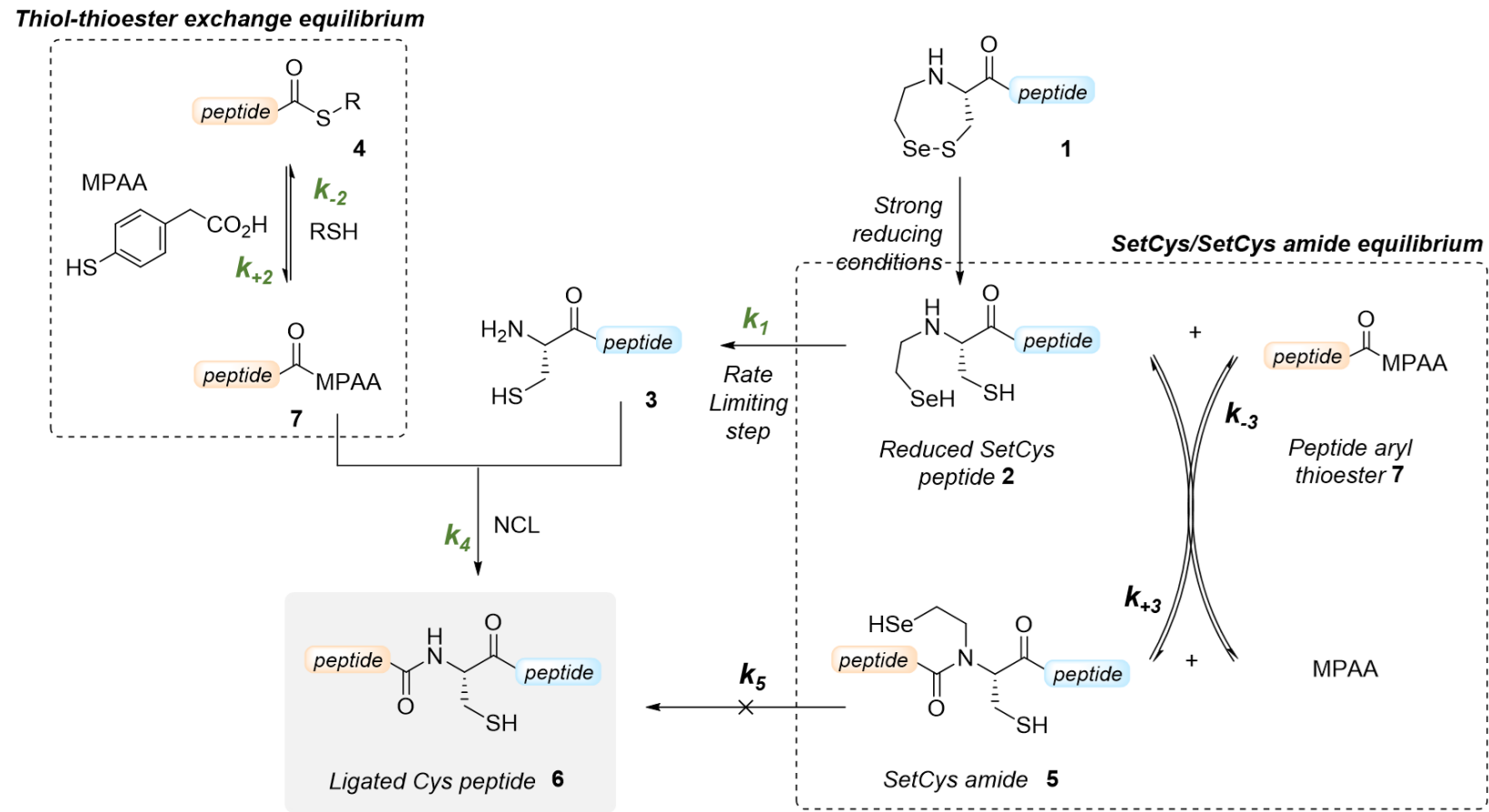


Figure 4. a) Ligation of $N$-(2-selanylethyl)cysteine peptides with peptide alkyl thioester in NCL standard conditions yields a product featuring a native peptide bond to cysteine; b) RP-HPLC monitoring of the conversion of peptides 5 (black triangles) and $\mathbf{6}$ (brown circles) throughout the course of the reaction. Fitting curves for each compound are represented by dashed lines; c) Proposed mechanistic model for the ligation of $\mathrm{N}$-(2-selanylethyl)cysteine peptides under strong reducing conditions. The rate constants were obtained by software-assisted numerical integration of rate equations (Kintek explorer $\left.{ }^{\mathrm{TM}}\right)$. 
a
$=\mathrm{N}$-terminal Cys<smiles>CC(=O)C1CS[Se]CCN1</smiles><smiles>[C-]=C(C)N1CCSCC1</smiles>
$\mathrm{C}=$ Alkyl thioester
Silent SetCys
Silent SEA<smiles>CC(=O)C(CS)NCC=S</smiles><smiles></smiles>
Reactive SetCys Reactive SEA

b

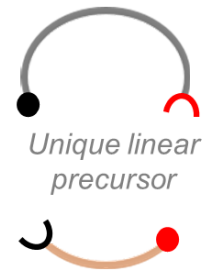

Linker

Reaction conditions: Step 1. MPAA, pH 7.2; Step 2 \& 3. TCEP addition, pH 5.5
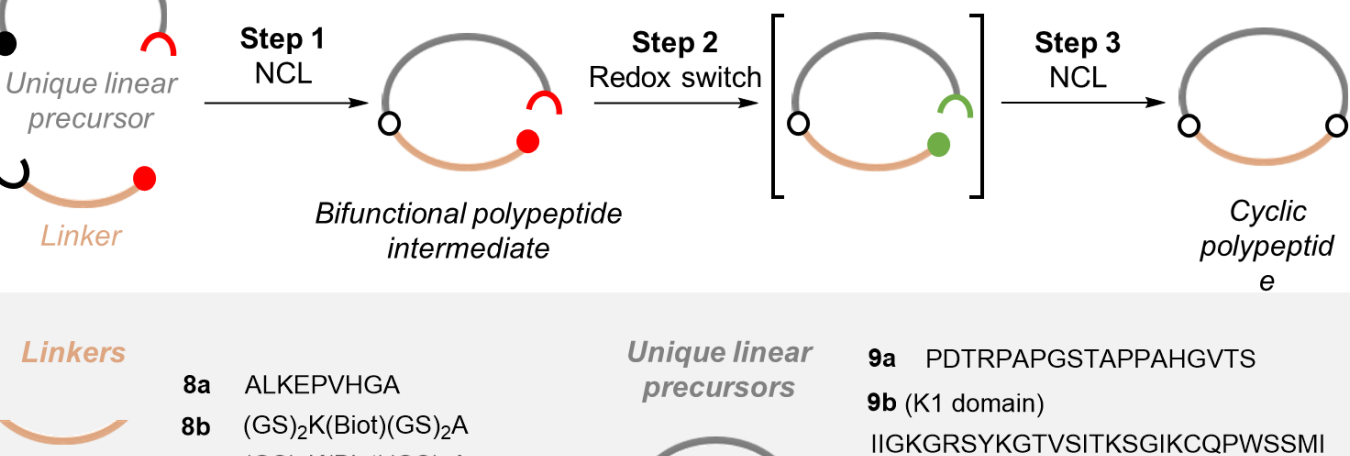
8a ALKEPVHGA
$8 \mathrm{~b} \quad(\mathrm{GS})_{2} \mathrm{~K}(\mathrm{Biot})(\mathrm{GS})_{2} \mathrm{~A}$
8c
$(\mathrm{GS})_{3} \mathrm{~K}(\mathrm{Biot})(\mathrm{GS})_{3} \mathrm{~A}$

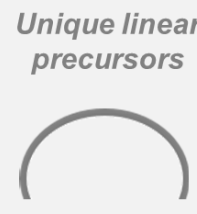

9a PDTRPAPGSTAPPAHGVTS

$9 \mathbf{b}$ (K1 domain)

IIGKGRSYKGTVSITKSGIKCQPWSSMI PHEHSFLPSSYRGKDLQENYCRNPRG EEGGPWCFTSNPEVRYEVCDIPQ
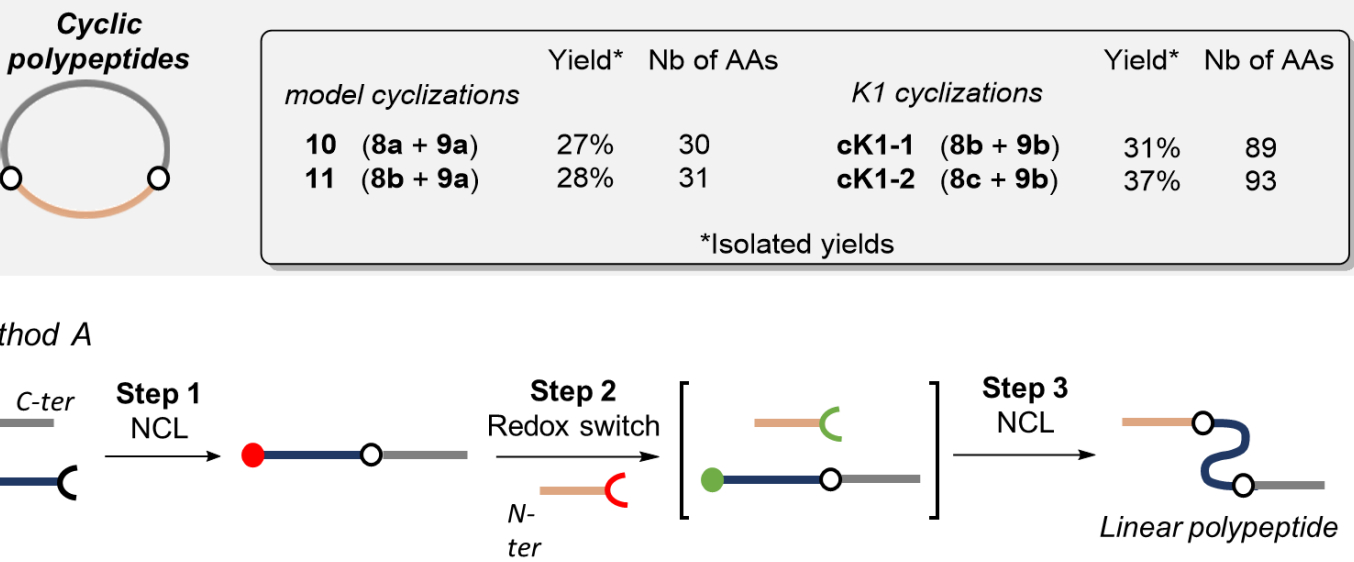

Method B

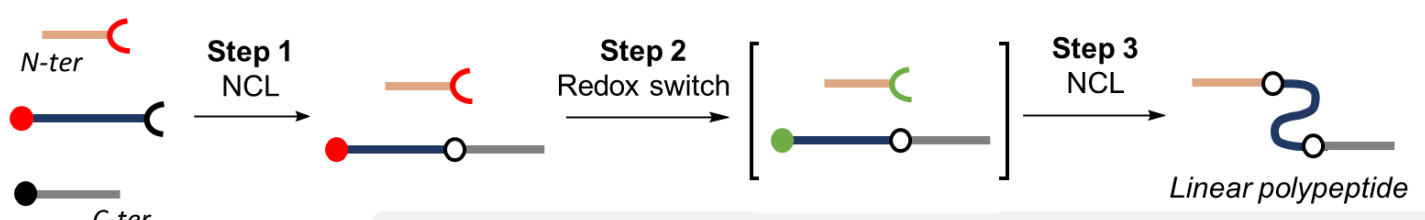

C-ter

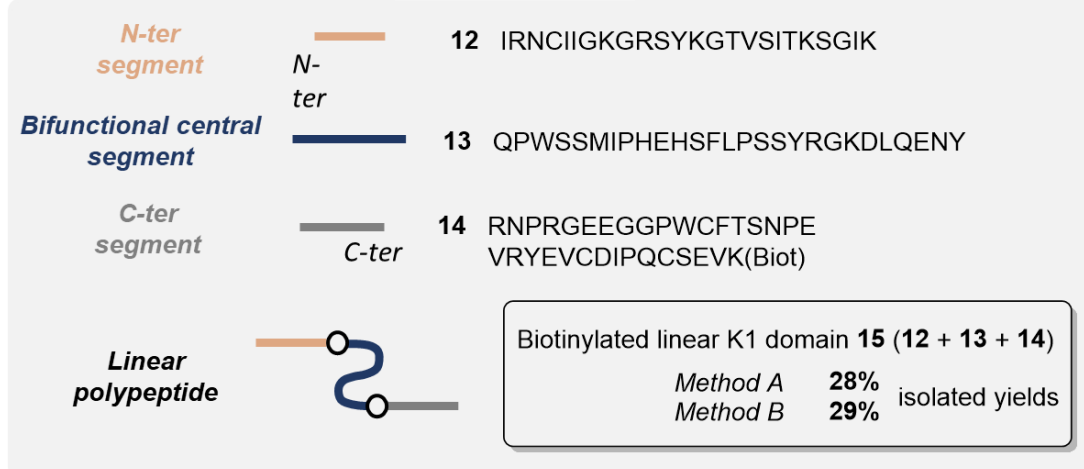


Figure 5. a) Legend; b) One-pot grafting of a peptide linker to a unique linear precursor yields cyclic polypeptides. Application to the total synthesis of cyclic and biotinylated analogs of HGF/SF K1 domain (cK1-1, cK1-2); c) One-pot synthesis of linear K1 domain. 
$\begin{array}{llllll}28 & 149 & 177 & 189 & 201 & 206\end{array}$ CIIGKGRSYKGTVSITKSGIKCQPWSSMIPHEHSFLPSSYRGKDLQENYCRNPRGEEGGPWCFTSNPEVRYEVCDIPQC

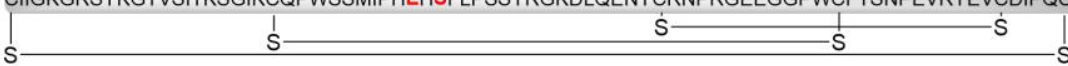

HGF/SF K1 domain

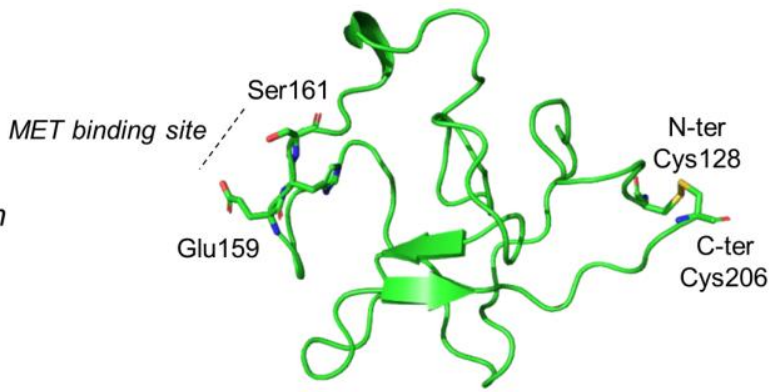

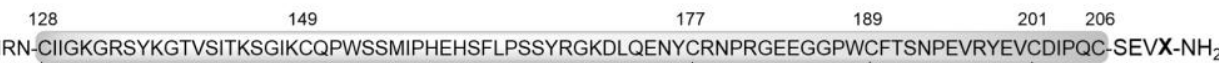

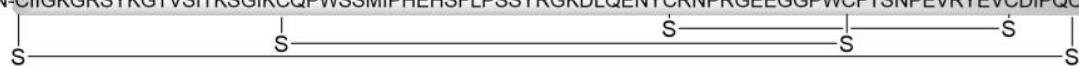

K1B: $\mathrm{X}=\mathrm{K}($ Biot $)$
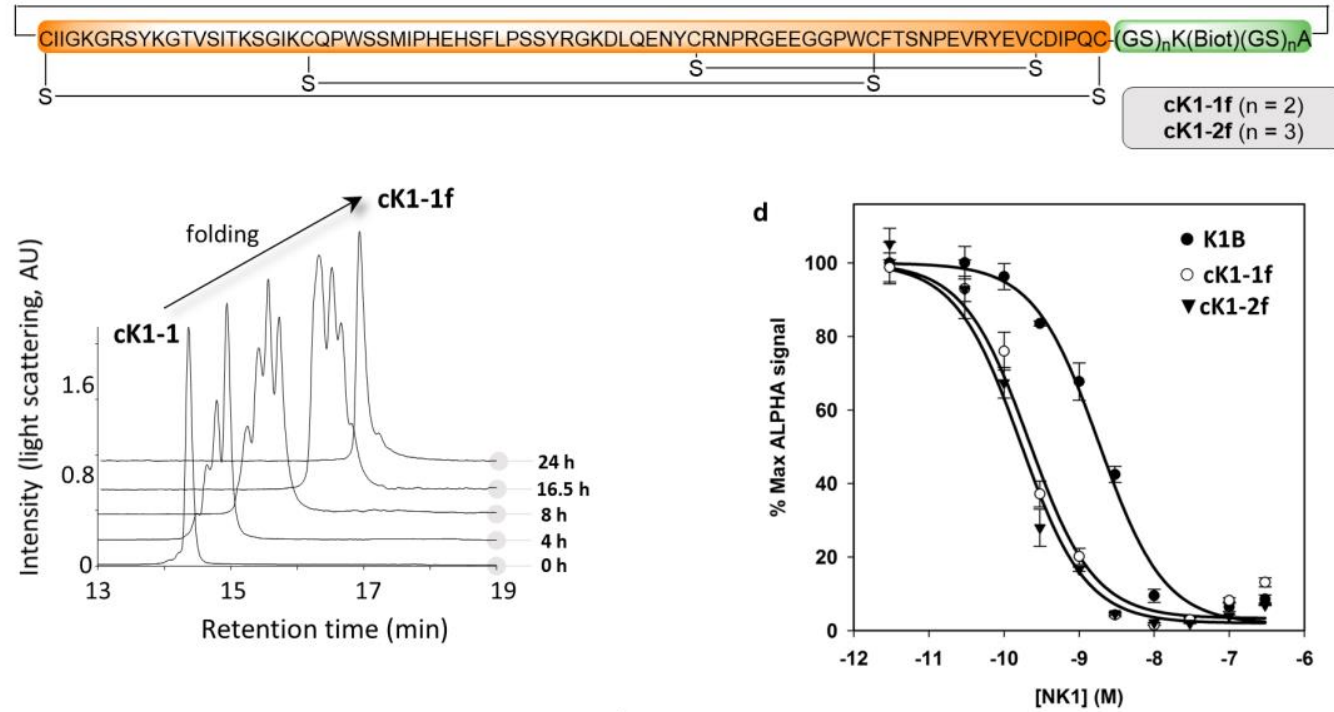

e

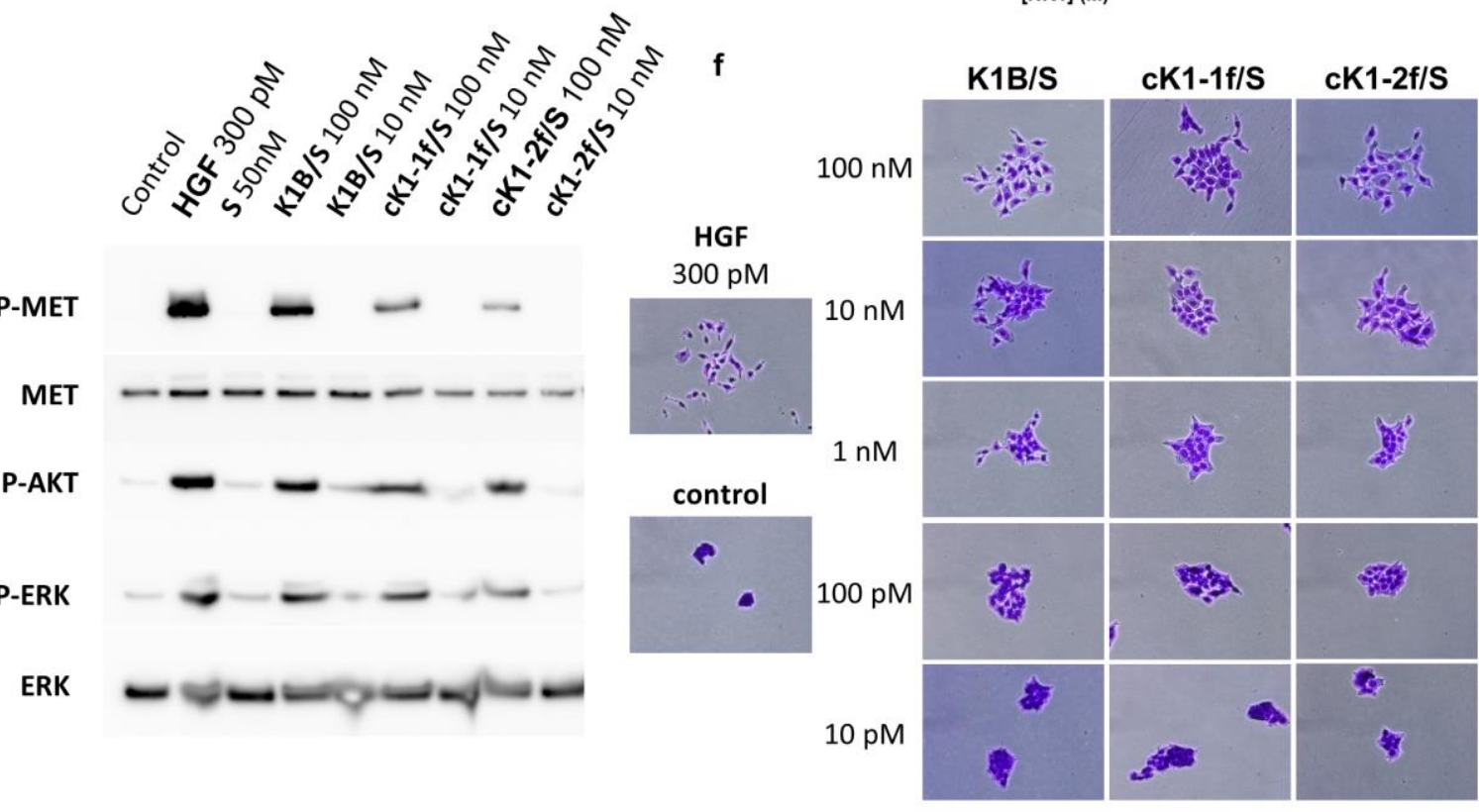


Figure 6. a) Primary and tertiary structure of HGF/SF K1 domain (pdb entry 1BHT). b) Biotinylated K1 analogs tested for their capacity to bind MET receptor and induce MET-specific phenotypes. The pattern of disulfide bonds determined experimentally corresponds to the native pattern found in $\mathrm{K} 1$ domain X-ray crystal structures. c) LC-MS monitoring of the folding of cK1-1 peptide into cK1-1f. d) Competitive AlphaScreen ${ }^{\circledR}$ assay with recombinant NK1 protein. K1B or cK1-1f or cK1-2f were mixed with increasing concentrations of NK1 and with extracellular MET domain fused with human IgG1-Fc (MET-Fc) and incubated with streptavidin AlphaScreen ${ }^{\circledR}$ donor beads and Protein A acceptor beads. e) HeLa cells were treated for 10 min with 300 pM mature HGF/SF (HGF), or with $10 \mathrm{nM} / 100 \mathrm{nM} \mathrm{K1/S}$, cK1-1f/S and cK1-2f/S. Cell lysates were then analyzed by specific total MET and ERK or phosphoMET, phospho-Akt and phospho-ERK Western blot; f) Cell scattering assay. Human Capan 1 isolated cell islets were incubated for $18 \mathrm{~h}$ in culture media with $300 \mathrm{pM}$ mature HGF/SF (HGF), or 100, 10, 1 nM and 100 and 10 pM K1B, cK1-1f and cK1-2f.

\section{References}

1. Dawson P. E., Muir T. W., Clark-Lewis I. \& Kent S. B. H. Synthesis of proteins by native chemical ligation. Science 266, 776-779 (1994).

2. Agouridas V., El Mahdi O., Diemer V., Cargoet M., Monbaliu J.-C. M. \& Melnyk O. Native chemical ligation and extended methods. Mechanisms, catalysis, scope and limitations. Chem. Rev. 12, 7328-7443 (2019).

3. Kulkarni S. S., Sayers J., Premdjee B. \& Payne R. J. Rapid and efficient protein synthesis through expansion of the native chemical ligation concept. Nat. Rev. Chem. 2, 0122 (2018).

4. Conibear A. C., Watson E. E., Payne R. J. \& Becker C. F. W. Native chemical ligation in protein synthesis and semi-synthesis. Chem. Soc. Rev. 47, 9046-9068 (2018).

5. Agouridas V., El Mahdi O., Cargoët M. \& Melnyk O. A statistical view of protein chemical synthesis using NCL and extended methodologies. Bioorg. Med. Chem. 25, 4938-4945 (2017).

6. Raibaut L., Ollivier N. \& Melnyk O. Sequential native peptide ligation strategies for total chemical protein synthesis. Chem. Soc. Rev. 41, 7001-7015 (2012).

7. Jbara M., Laps S., Morgan M., Kamnesky G., Mann G., Wolberger C. et al. Palladium prompted ondemand cysteine chemistry for the synthesis of challenging and uniquely modified proteins. Nat. Commun. 9, 3154 (2018).

8. Maity S. K., Mann G., Jbara M., Laps S., Kamnesky G. \& Brik A. Palladium-assisted removal of a solubilizing tag from a Cys side chain to facilitate peptide and protein synthesis. Org. Lett. 18, 3026-3029 (2016).

9. Maity S. K., Jbara M., Laps S. \& Brik A. Efficient palladium-assisted one-pot deprotection of (acetamidomethyl)cysteine following native chemical ligation and/or desulfurization to expedite chemical protein synthesis. Angew. Chem. Int. Ed. 55, 8108-8112 (2016).

10. Jbara M., Maity S. K., Seenaiah M. \& Brik A. Palladium mediated rapid deprotection of N-terminal cysteine under native chemical ligation conditions for the efficient preparation of synthetically challenging proteins. J. Am. Chem. Soc. 138, 5069-5075 (2016).

11. Reddy P. S., Dery S. \& Metanis N. Chemical synthesis of proteins with non-strategically placed cysteines using selenazolidine and selective deselenization. Angew. Chem. Int. Ed. 55, 992-995 (2015).

12. Poole L. B. The basics of thiols and cysteines in redox biology and chemistry. Free Radic. Biol. Med. 80, 148-157 (2015).

13. Klomsiri C., Karplus P. A. \& Poole L. B. Cysteine-based redox switches in enzymes. Antioxid. Redox Signal. 14, 1065-1077 (2011). 
14. Greene T. W. in Protection for the Thiol Group (eds Wuts P. G. M.) 837-894 (Wiley, 2014).

15. Lees W. J. \& Whitesides G. M. Equilibrium constants for thiol-disulfide interchange reactions: a coherent, corrected set. J. Org. Chem. 58, 642-647 (1993).

16. Reich H. J. \& Cohen M. L. Organoselenium chemistry. Dealkylation of amines with benzeneselenol. J. Org. Chem. 44, 3148-3151 (1979).

17. Krief A. \& Hevesi L. Organoselenium Chemistry I. Functional Group Transformations (Springer-Verlag, 1988).

18. Ouyang K., Hao W., Zhang W.-X. \& Xi Z. Transition-Metal-Catalyzed Cleavage of C-N Single Bonds. Chem. Rev. 115, 12045-12090 (2015).

19. Loibl S. F., Harpaz Z. \& Seitz O. A type of auxiliary for native chemical peptide ligation beyond cysteine and glycine junctions. Angew. Chem. Int. Ed. 54, 15055-15059 (2015).

20. Yin H., Lu D., Wang S. \& Wang P. Development of Powerful Auxiliary-Mediated Ligation To Facilitate Rapid Protein Assembly. Org. Lett. 21, 5138-5142 (2019).

21. Dawson P. E., Churchill M. J., Ghadiri M. R. \& Kent S. B. H. Modulation of reactivity in native chemical ligation through the use of thiol additives. J. Am. Chem. Soc. 119, 4325-4329 (1997).

22. Johnson E. C. \& Kent S. B. H. Insights into the mechanism and catalysis of the native chemical ligation reaction. J. Am. Chem. Soc. 128, 6640-6646 (2006).

23. Melnyk O. \& Agouridas V. From protein total synthesis to peptide transamidation and metathesis: playing with the reversibility of $N, S$-acyl or N,Se-acyl migration reactions. Curr. Opin. Chem. Biol. 22, 137145 (2014).

24. Ruff Y., Garavini V. \& Giuseppone N. Reversible native chemical ligation: a facile access to dynamic covalent peptides. J. Am. Chem. Soc. 136, 6333-6339 (2014).

25. Reich H. J. \& Hondal R. J. Why Nature Chose Selenium. ACS Chem. Biol. 11, 821-841 (2016).

26. Rohde H., Schmalisch J., Harpaz Z., Diezmann F. \& Seitz O. Ascorbate as an alternative to thiol additives in native chemical ligation. ChemBioChem 12, 1396-1400 (2011).

27. Dery S., Reddy P. S., Dery L., Mousa R., Dardashti R. N. \& Metanis N. Insights into the deselenization of selenocysteine into alanine and serine. Chem. Sci. 6, 6207-6212 (2015).

28. Metanis N., Keinan E. \& Dawson P. E. Traceless ligation of cysteine peptides using selective deselenization. Angew. Chem. Int. Ed. 49, 7049-7053 (2010).

29. Wan Q. \& Danishefsky S. J. Free-radical-based, specific desulfurization of cysteine: a powerful advance in the synthesis of polypeptides and glycopolypeptides. Angew. Chem. Int. Ed. 46, 9248-9252 (2007).

30. Yokoyama A., Sakurai H. \& Tanaka H. Syntheses of related compounds of selenocysteamine and their complex formation with metal ions. Chem. Pharm. Bull. 19, 1089-1094 (1971).

31. Jencks W. P. \& Regenstein J. in Ionization constants of acids and bases (eds Lundblad R. L. \& MacDonald F. M.) 595-635 (CRC, 2010).

32. Tanini D. \& Capperucci A. Ring opening reactions of heterocycles with selenium and tellurium nucleophiles. New J. Chem. 43, 11451-11468 (2019).

33. Ollivier N., Blanpain A., Boll E., Raibaut L., Drobecq H. \& Melnyk O. Selenopeptide transamidation and metathesis. Org. Lett. 16, 4032-4035 (2014).

34. Camarero J. A., Fushman D., Sato S., Giriat I., Cowburn D., Raleigh D. P. et al. Rescuing a destabilized protein fold through backbone cyclization. J. Mol. Biol. 308, 1045-1062 (2001).

35. Iwai H. \& Pluckthun A. Circular beta-lactamase: stability enhancement by cyclizing the backbone. FEBS Lett. 459, 166-172 (1999).

36. Camarero J. A. \& Muir T. W. Biosynthesis of a head-to-tail cyclized protein with improved biological activity. J. Am. Chem. Soc. 121, 5597-5598 (1999).

37. Craik D. J. Chemistry. Seamless proteins tie up their loose ends. Science 311, 1563-1564 (2006).

38. Craik D. J., Fairlie D. P., Liras S. \& Price D. The future of peptide-based drugs. Chem. Biol. Drug Des. 81, 136-147 (2013).

39. Krishna M. M. \& Englander S. W. The N-terminal to C-terminal motif in protein folding and function. Proc. Natl. Acad. Sci. U. S. A. 102, 1053-1058 (2005).

40. Mulvenna J. P., Wang C. \& Craik D. J. CyBase: a database of cyclic protein sequence and structure. Nucleic Acids Res. 34, D192-D194 (2006).

41. White C. J. \& Yudin A. K. Contemporary strategies for peptide macrocyclization. Nat. Chem. 3, 509-524 (2011).

42. Camarero J. A. \& Muir T. W. Chemoselective backbone cyclization of unprotected peptides. Chem. Commun., 1369-1370 (1997). 
43. Ollivier N., Dheur J., Mhidia R., Blanpain A. \& Melnyk O. Bis(2-sulfanylethyl)amino native peptide ligation. Org. Lett. 12, 5238-5241 (2010).

44. Boll E., Drobecq H., Ollivier N., Blanpain A., Raibaut L., Desmet R. et al. One-pot chemical synthesis of small ubiquitin-like modifier (SUMO) protein-peptide conjugates using bis(2-sulfanylethyl)amido peptide latent thioester surrogates Nat. Protoc. 10, 269-292 (2015).

45. Ollivier N., Vicogne J., Vallin A., Drobecq H., Desmet R., El-Mahdi O. et al. A one-pot three-segment ligation strategy for protein chemical synthesis. Angew. Chem. Int. Ed. 51, 209-213 (2012).

46. Simonneau C., Berenice L., Mougel A., Adriaenssens E., Paquet C., Raibaut L. et al. Semi-synthesis of a HGF/SF kringle one (K1) domain scaffold generates a potent in vivo MET receptor agonist. Chem. Sci. 6, 2110-2121 (2015).

47. Ultsch M., Lokker N. A., Godowski P. J. \& de Vos A. M. Crystal structure of the NK1 fragment of human hepatocyte growth factor at 2.0 A resolution. Structure 6, 1383-1393 (1998).

48. Mekki M. S., Mougel A., Vinchent A., Paquet C., Copin M. C., Leroy C. et al. Hypoxia leads to decreased autophosphorylation of the MET receptor but promotes its resistance to tyrosine kinase inhibitors. Oncotarget 9, 27039-27058 (2018). 


\section{Supporting Information for}

\section{A cysteine selenosulfide redox switch for protein chemical synthesis}

Vincent Diemer, Nathalie Ollivier, Bérénice Leclercq, Hervé Drobecq, Jérôme Vicogne, Vangelis Agouridas*, Oleg Melnyk*

University of Lille, CNRS, Institut Pasteur de Lille, UMR CNRS 8204, INSERM U1019, Centre

d'Immunité et d'Infection de Lille, F-59000 Lille, France

Corresponding author:

Dr Oleg Melnyk, E-mail : oleg.melnyk@ibl.cnrs.fr

Dr Vangelis Agouridas, E-mail: vangelis.agouridas@ibl.cnrs.fr

Website: http://olegmelnyk.cnrs.fr

CBF group

Institut de Biologie de Lille

1 rue du Pr Calmette, CS 50447, 59021 Lille cedex, France 


\section{Table of contents}

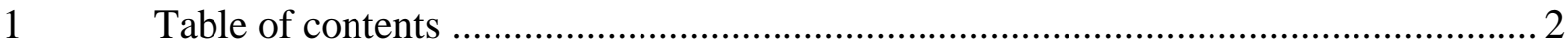

2 Synthesis of Fmoc-protected SetCys amino acid …........................................... 5

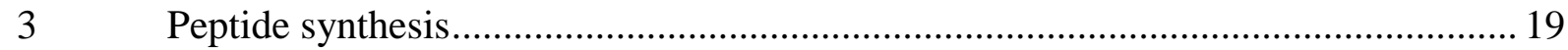

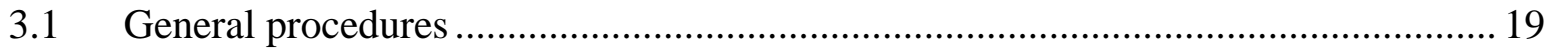

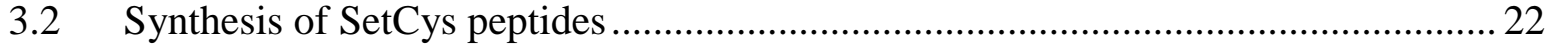

SetCys peptide used for exploring SetCys reactivity (Figure $2 \&$ Figure 3) ................... 22

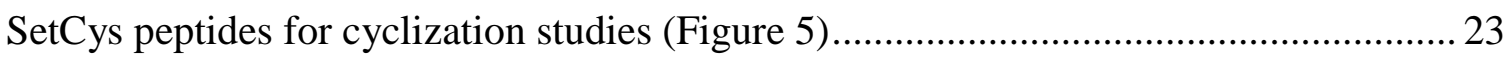

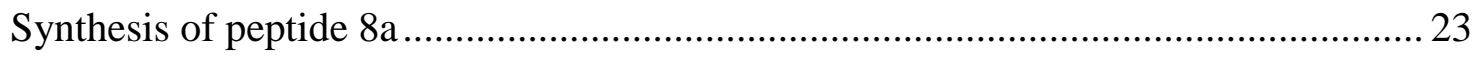

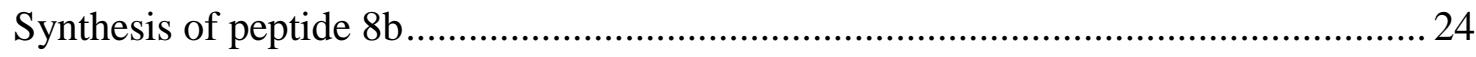

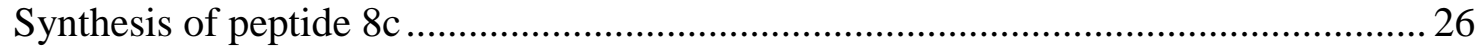

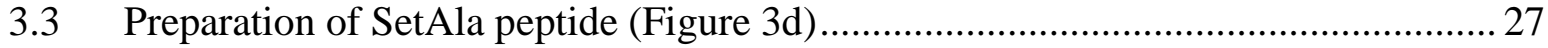

3.4 Synthesis of SEA ${ }^{\text {off }}$ peptide segments for cyclization studies (Figure 5) ................... 32

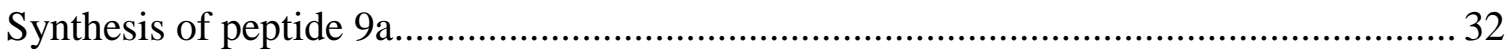

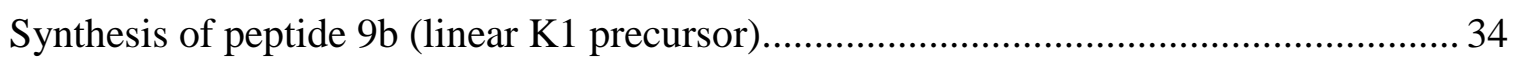

Synthesis of the peptide segments for the assembly of linear K1 peptide 9b .............. 35

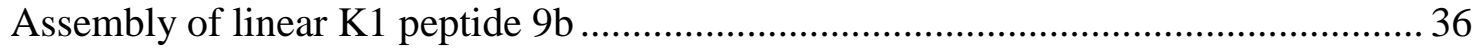

4 Stability and reactivity of SetCys unit as a function of the reducing power of the

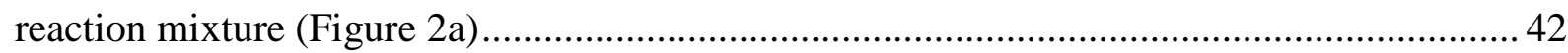

4.1 Stability of SetCys under low (property 1) or strong (property 4) reducing conditions 42

4.2 Stability of SetCys under strong (property 4) reducing conditions. Importance of

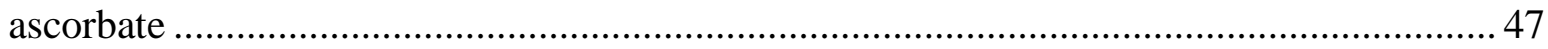

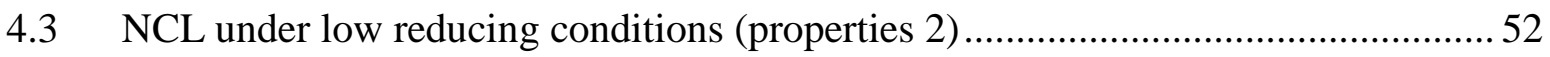

4.4 SetCys is silent during NCL under low reducing conditions (property 3) ................57

4.4.1 Competitive ligation experiment involving a Cys and SetCys peptide..............57

4.4.2 Compatibility of SetCys with Cys-rich peptides ............................................. 61

4.5 NCL under strong reducing conditions (peptide alkyl thioester, TCEP/ascorbate,

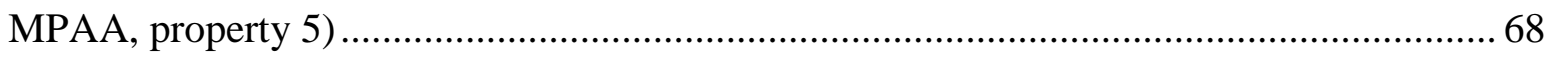

4.6 NCL under strong reducing conditions (peptide aryl thioester, TCEP/ascorbate, no

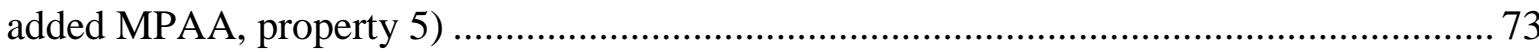


4.7 NCL under strong reducing conditions (peptide alkyl thioester, DTT, MPAA, property 5)

5 Stability and reactivity of N-(2-sulfanylethyl)Cys (SutCys) unit as a function of the

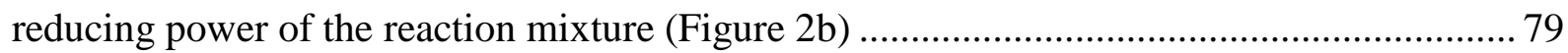

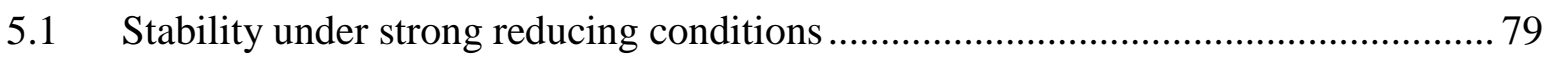

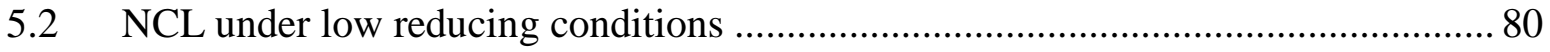

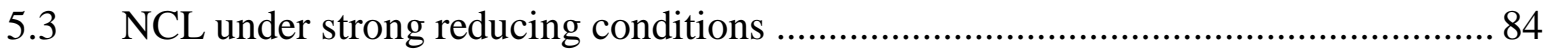

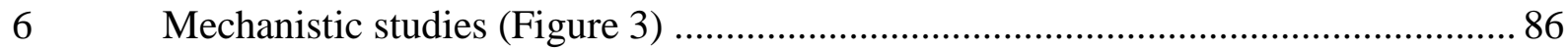

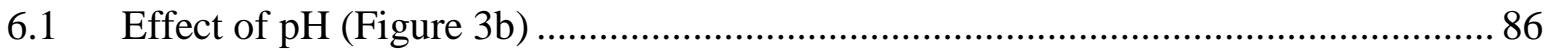

6.2 Effect of Cys thiol: control experiment with a SetAla peptide (Figure 3d) ............. 87

6.2.1 Decomposition of SetAla peptide into Ala peptide under strong reducing

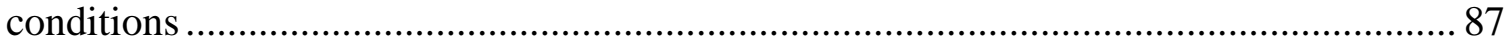

6.2.2 Ligation of SetAla peptide under strong reducing conditions ........................... 90

$7 \quad$ Kinetic model of SetCys-mediated NCL under strong reducing conditions (Figure 4) 92

7.1 Rate constants for a classical NCL $\left(\mathrm{k}_{+2}, \mathrm{k}_{-2}\right.$ and $\mathrm{k}_{4}$, see Figure $\left.4 \mathrm{c}\right)$......................... 93

7.2 Rate constant for the loss of the $N$-selenoethyl appendage $\left(\mathrm{k}_{1}\right.$, Figure $\left.4 \mathrm{c}\right) \ldots \ldots \ldots \ldots . . .95$

7.3 Kinetic model for SetCys-mediated NCL (Figure 4b,c) ........................................ 95

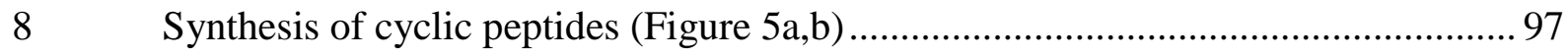

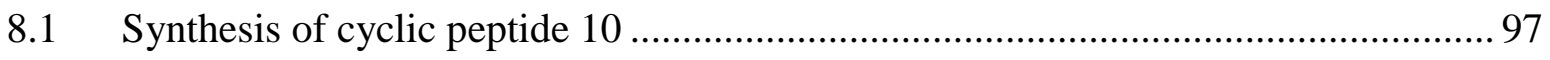

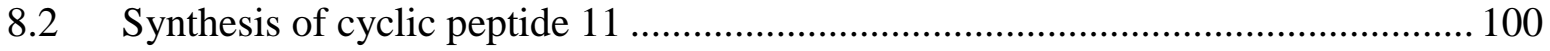

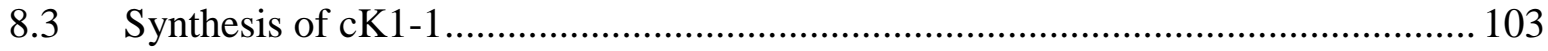

8.3.1 Characterization of the cyclic backbone structure of cK1-1 .......................... 105

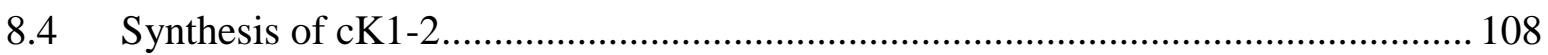

8.4.1 Characterization of the cyclic backbone structure of cK1-2 _......................... 111

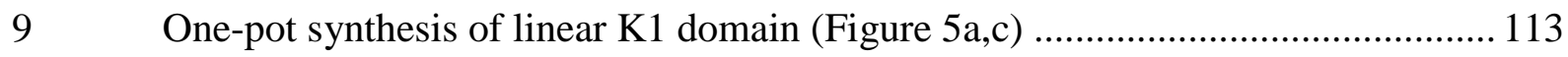

9.1 Preparation of the starting peptide segments ................................................. 113

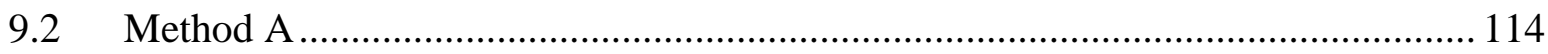

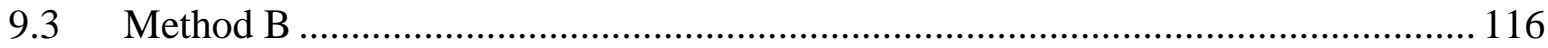

9.4 Characterization of linear K1 polypeptide........................................................... 119

10 Folding, characterization and biological activity of cK1-1 and cK1-2 (Figure 6) .. 123

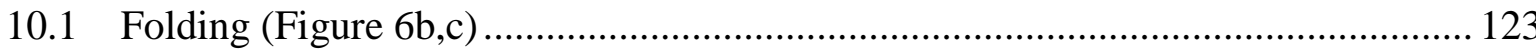




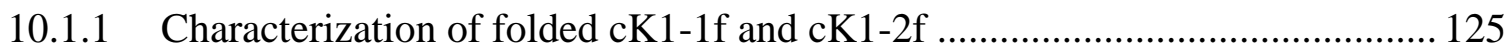

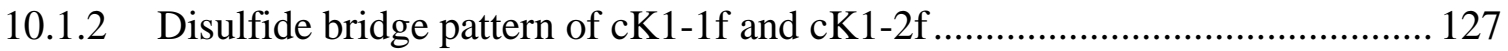

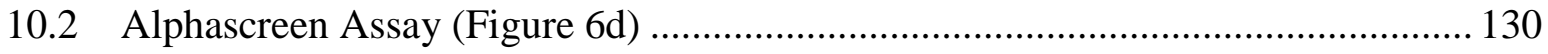

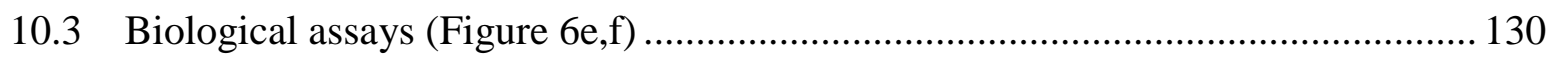

10.3.1 Cell-based assay for MET signaling induced by cK1-1f and cK1-2f ............. 130

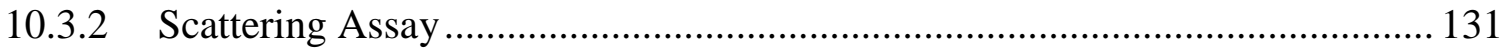

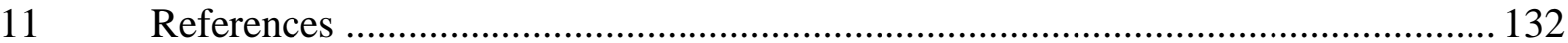




\section{Synthesis of Fmoc-protected SetCys amino acid}

Fmoc-protected SetCys amino acid SI8 was prepared as described below in Figure S 1.

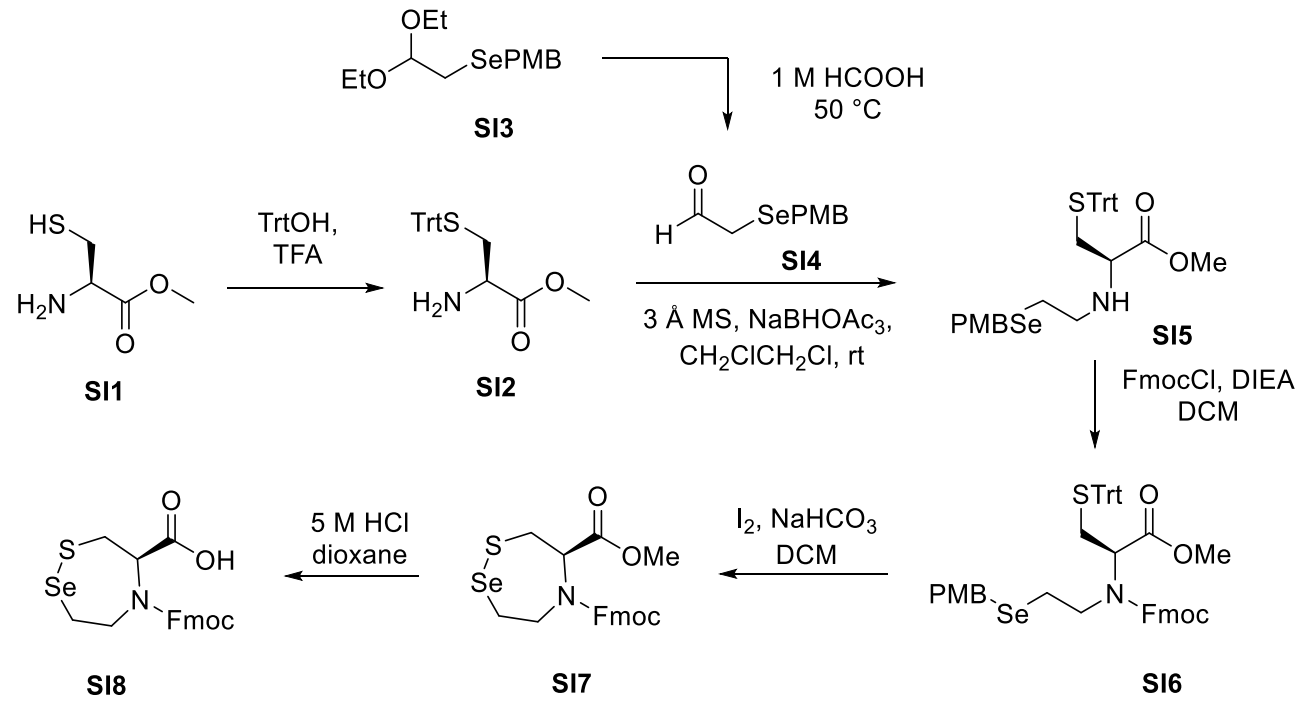

Figure S 1. Synthesis of the Fmoc-protected SetCys amino acid.

(2,2-Diethoxyethyl)(4-methoxybenzyl)selenide was prepared according to Abbas et al. ${ }^{1}$

\subsection{Synthesis of Synthesis of H-Cys(Trt)-OMe}

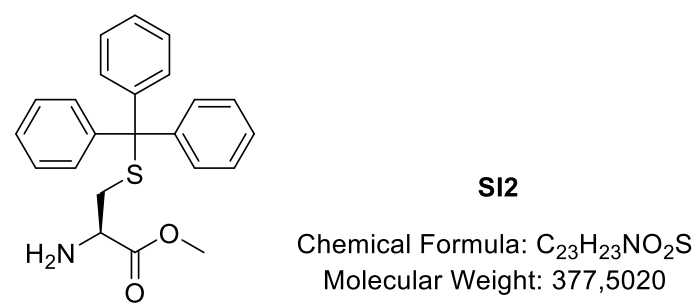

To a solution of cysteine methyl ester SI1 (856 mg, $5 \mathrm{mmol}$ ) in TFA (5 mL) was added triphenylmethanol (1.43 g, $5.5 \mathrm{mmol})$ and the mixture was stirred at RT for $5 \mathrm{~h}$. After evaporation of the TFA, the residue was dissolved in $\mathrm{MeOH}$ and the mixture was stirred at RT until the yellow color disappeared. The methanol was then evaporated under reduced pressure and the residue was suspended in $0.25 \mathrm{M} \mathrm{K}_{2} \mathrm{CO}_{3}(30 \mathrm{~mL})$. The obtained aqueous layer was extracted with $\mathrm{Et}_{2} \mathrm{O}(2 \times 20 \mathrm{~mL})$ and the combined organic layers were dried over $\mathrm{MgSO}_{4}$. After evaporation of the solvent, purification of the crude by column chromatography (DCM/MeOH 98:2) provided the S-trityl protected amino acid SI2 (1.73 g) as a viscous oil with $91 \%$ yield.

NMR data are in agreement with the literature. ${ }^{2}$

${ }^{1} \mathbf{H}$ NMR $\left(300 \mathrm{MHz}, \mathrm{CDCl}_{3}\right) \delta$ 7.41-7.44 (m, 6H), 7.18-7.31 (m, 9H), 3.65 (s, 3H), 3.20 (dd, $J$ $=4.8$ and $7.8 \mathrm{~Hz}, 1 \mathrm{H}), 2.59(\mathrm{dd}, J=4.8$ and $12.4 \mathrm{~Hz}, 1 \mathrm{H}), 2.46(\mathrm{~d}, J=7.8$ and $12.4 \mathrm{~Hz}, 1 \mathrm{H})$ 
ppm. ${ }^{13} \mathrm{C}$ NMR $\left(75 \mathrm{MHz}, \mathrm{CDCl}_{3}\right) \delta 174.3(\mathrm{C}), 144.6(3 \times \mathrm{CH}), 129.7(6 \times \mathrm{CH}), 128.1(6 \times$ $\mathrm{CH}), 126.9(3 \times \mathrm{CH}), 66.9(\mathrm{C}), 53.9(\mathrm{CH}), 52.3\left(\mathrm{CH}_{3}\right), 37.2\left(\mathrm{CH}_{2}\right) \mathrm{ppm}$.

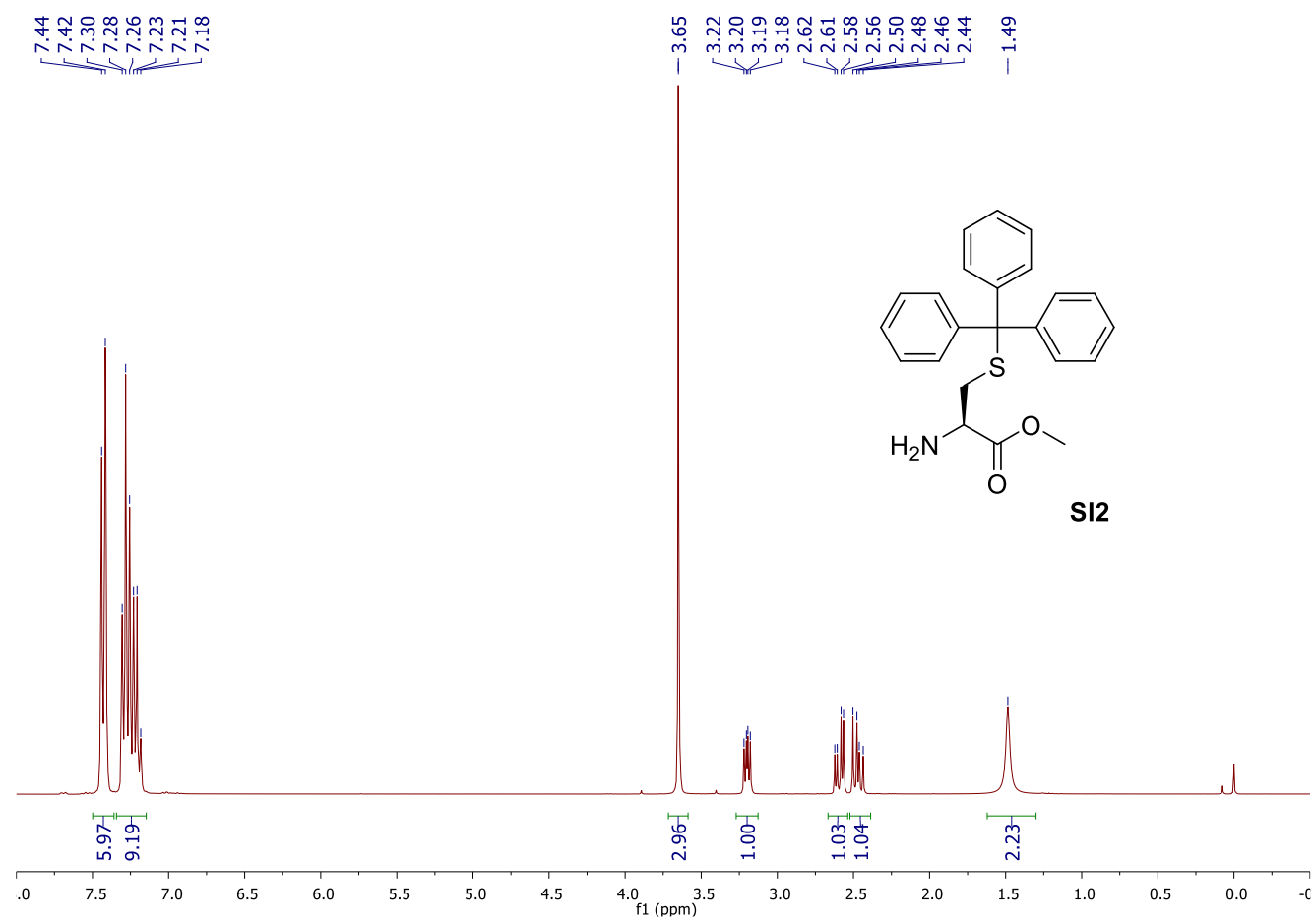

Figure S 2. ${ }^{1} \mathrm{H}$ NMR (300 MHz) spectrum of H-Cys(Trt)-OMe SI2 (CDCl 3 , $\left.293 \mathrm{~K}\right)$.

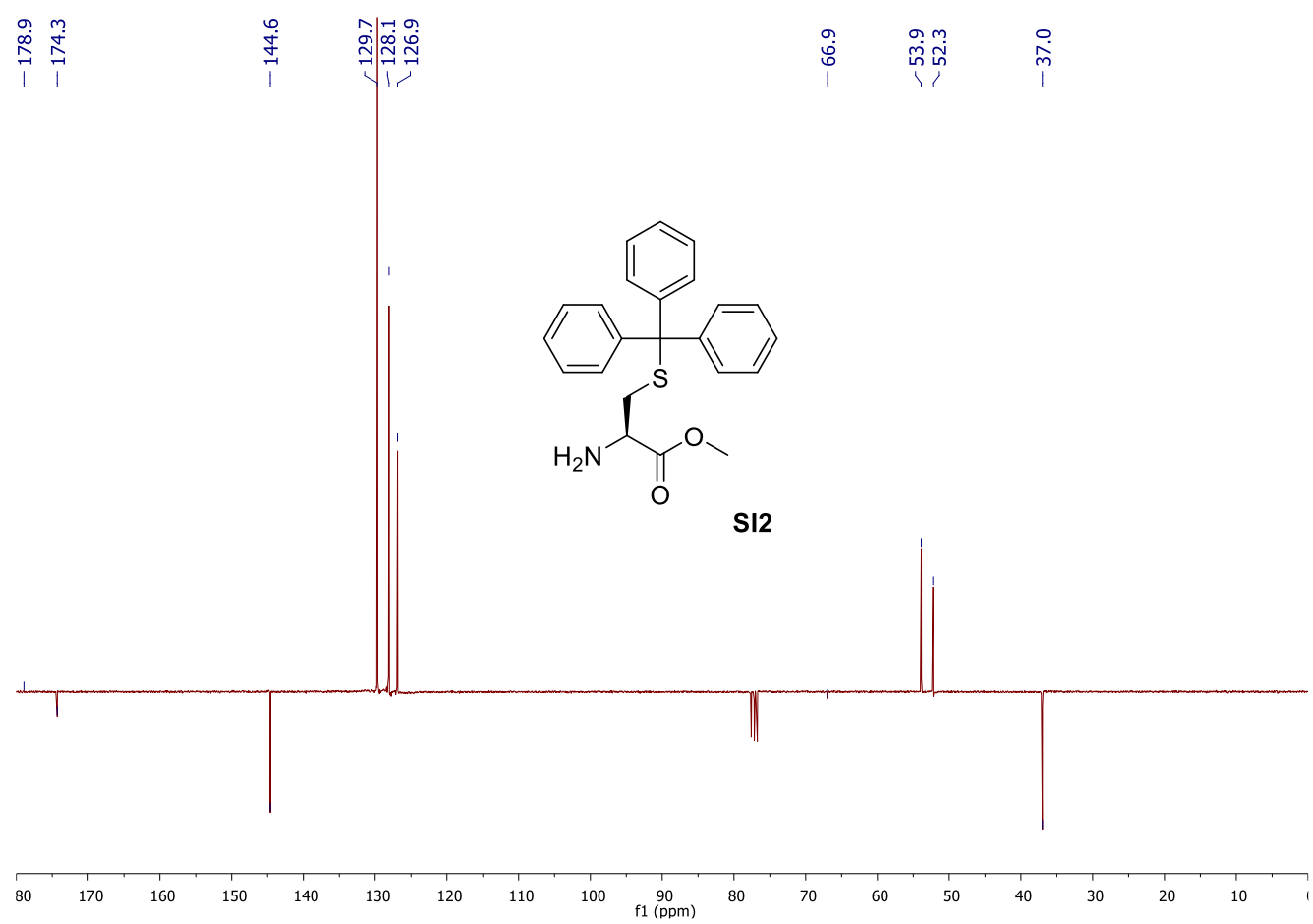

Figure S 3. ${ }^{13} \mathrm{C}$ JMOD NMR (75 MHz) spectrum of H-Cys(Trt)-OMe SI2 (CDCl $\left.3,293 \mathrm{~K}\right)$. 


\subsection{Synthesis of $N$-[PMBSe-(CH2)2]-Cys(Trt)-OMe}

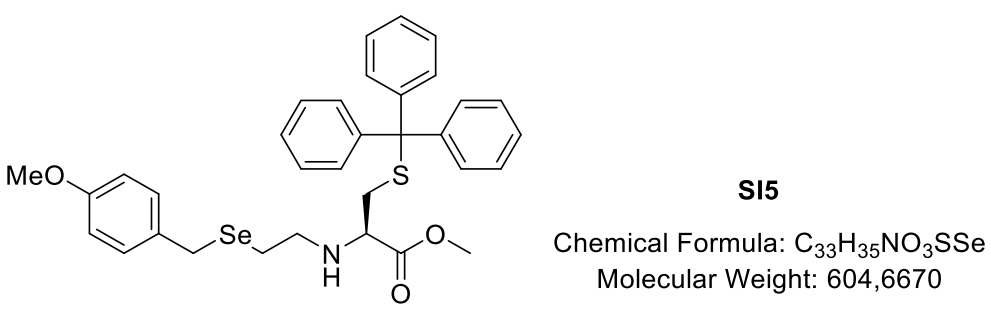

(2,2-Diethoxyethyl)(4-methoxybenzyl)selenide SI3 (1.18 g, $3.72 \mathrm{mmol}$ ) was suspended in $1 \mathrm{M}$ formic acid $(15 \mathrm{~mL})$ and the mixture was heated overnight at $50{ }^{\circ} \mathrm{C}$. Water $(50 \mathrm{~mL})$ was added and the aqueous layer was extracted with $\mathrm{Et}_{2} \mathrm{O}(2 \times 50 \mathrm{~mL})$. The combined organic extracts were washed with water $(50 \mathrm{~mL})$ and dried over $\mathrm{MgSO}_{4}$. After evaporation of the solvent under reduced pressure, aldehyde SI4 was dissolved in anhydrous 1,2-dichloroethane $(50 \mathrm{~mL})$ under argon. Activated powdered $3 \AA$ molecular sieves ( $4 \mathrm{~g})$, sodium triacetoxyborohydride $(1.10 \mathrm{~g}$, $5.21 \mathrm{mmol}$ ) and a solution of H-Cys(Trt)-OMe SI2 (1.41 g, $3.72 \mathrm{mmol})$ in DCE (35 mL) were successively added to the solution of aldehyde. After $18 \mathrm{~h}$ stirring at RT, the reaction mixture was filtered on a Büchner funnel and the solid was washed with additional portions of DCM. The filtrate was evaporated under reduced pressure. Then $0.5 \mathrm{M} \mathrm{K}_{2} \mathrm{CO}_{3}(50 \mathrm{~mL})$ was added to the residue and the aqueous layer was extracted with DCM $(3 \times 50 \mathrm{~mL})$. The combined organic layers were dried over $\mathrm{MgSO}_{4}$ and the solvent was evaporated under reduced pressure. Purification of the crude mixture by column chromatography (cyclohexane/ethyl acetate 80:20) afforded the N-alkylated amino acid SI5 as a clear yellow oil $(1.94 \mathrm{~g}, 86 \%)$.

${ }^{1} \mathbf{H}$ NMR $\left(300 \mathrm{MHz}, \mathrm{CDCl}_{3}\right) \delta 7.41(\mathrm{~d}, J=7.4 \mathrm{~Hz}, 6 \mathrm{H}), 7.19-7.32(\mathrm{~m}, 9 \mathrm{H}), 7.17(\mathrm{~d}, J=8.6 \mathrm{~Hz}$, $2 \mathrm{H}), 6.79(\mathrm{~d}, J=8.6 \mathrm{~Hz}, 2 \mathrm{H}), 3.77(\mathrm{~s}, 3 \mathrm{H}), 3.71(\mathrm{~s}, 2 \mathrm{H}), 3.66(\mathrm{~s}, 3 \mathrm{H}), 2.99(\mathrm{t}, J=6.5 \mathrm{~Hz}, 1 \mathrm{H})$, 2.37-2.74 (m, 6H), 1.44-1.91 (m, 1H) ppm. $\left.{ }^{13} \mathbf{C ~ N M R ~ ( 7 5 ~ M H z , ~} \mathrm{CDCl}_{3}\right) \delta 173.5(\mathrm{C}), 158.4(\mathrm{C})$, $144.6(3 \times \mathrm{C}), 131.1(\mathrm{C}), 129.9(2 \times \mathrm{CH}), 129.6(6 \times \mathrm{CH}), 127.9(6 \times \mathrm{CH}), 126.7(3 \times \mathrm{CH})$, $113.9(2 \times \mathrm{CH}), 66.8(\mathrm{C}), 60.2(\mathrm{CH}), 55.2\left(\mathrm{CH}_{3}\right), 51.9\left(\mathrm{CH}_{3}\right), 47.3\left(\mathrm{CH}_{2}\right), 34.6\left(\mathrm{CH}_{2}\right), 26.3$ $\left(\mathrm{CH}_{2}\right), 23.9\left(\mathrm{CH}_{2}\right)$ ppm. IR (ATR, $\left.\mathrm{cm}^{-1}\right)$ 3029, 2949, 1736, 1698, 1243, 1172, 738. HRMS

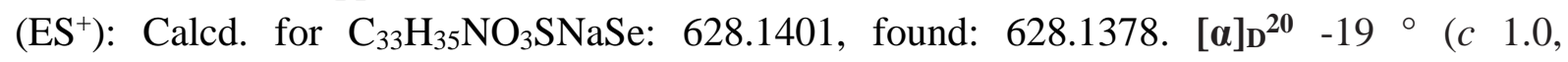
chloroform). 


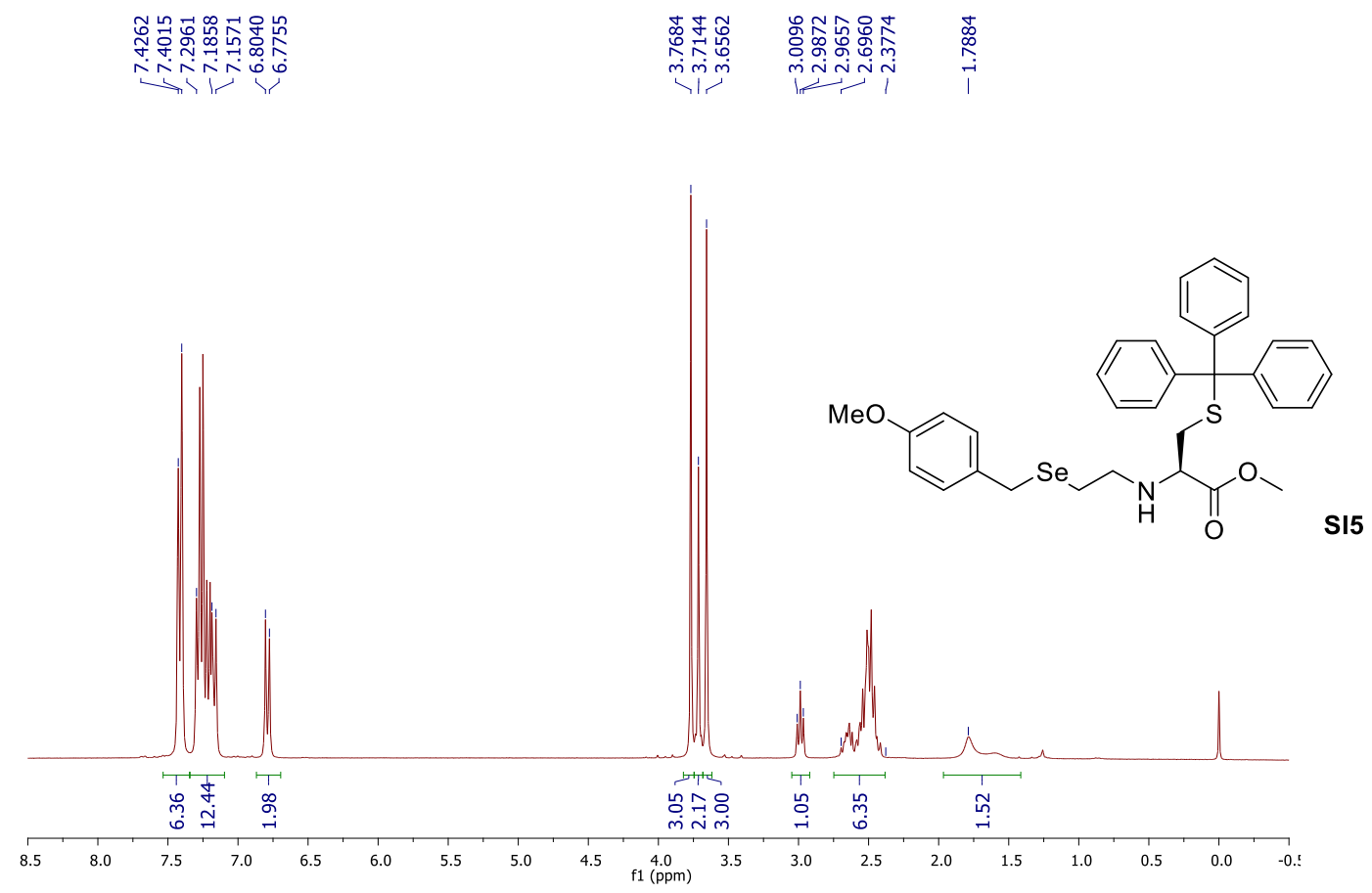

Figure S 4. ${ }^{1} \mathrm{H}$ NMR (300 MHz) spectrum of compound SI5 $\left(\mathrm{CDCl}_{3}, 293 \mathrm{~K}\right)$.

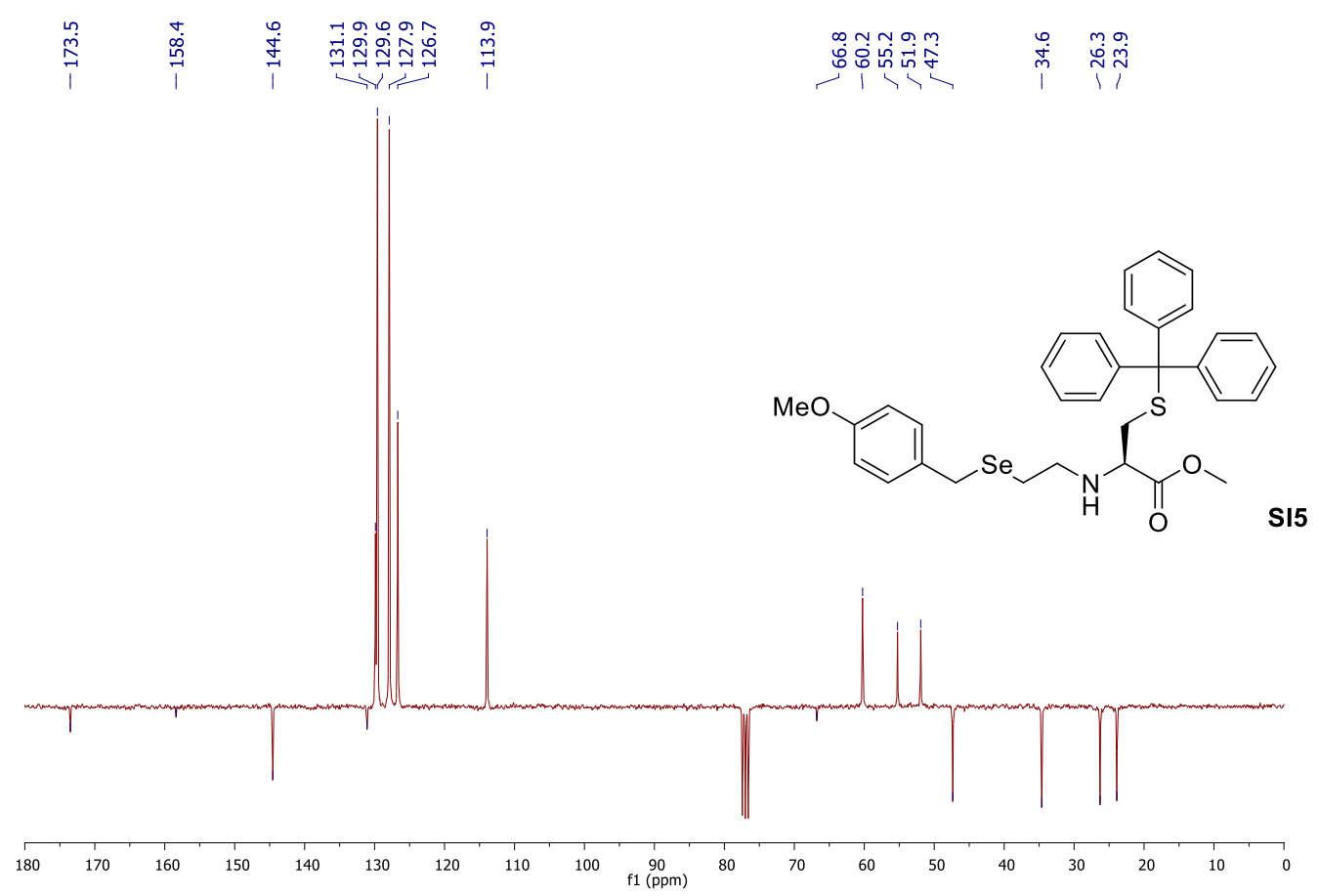

Figure S 5. ${ }^{13} \mathrm{C}$ JMOD NMR (75 MHz) spectrum of compound $\mathbf{S I 5}\left(\mathrm{CDCl}_{3}, 293 \mathrm{~K}\right)$. 


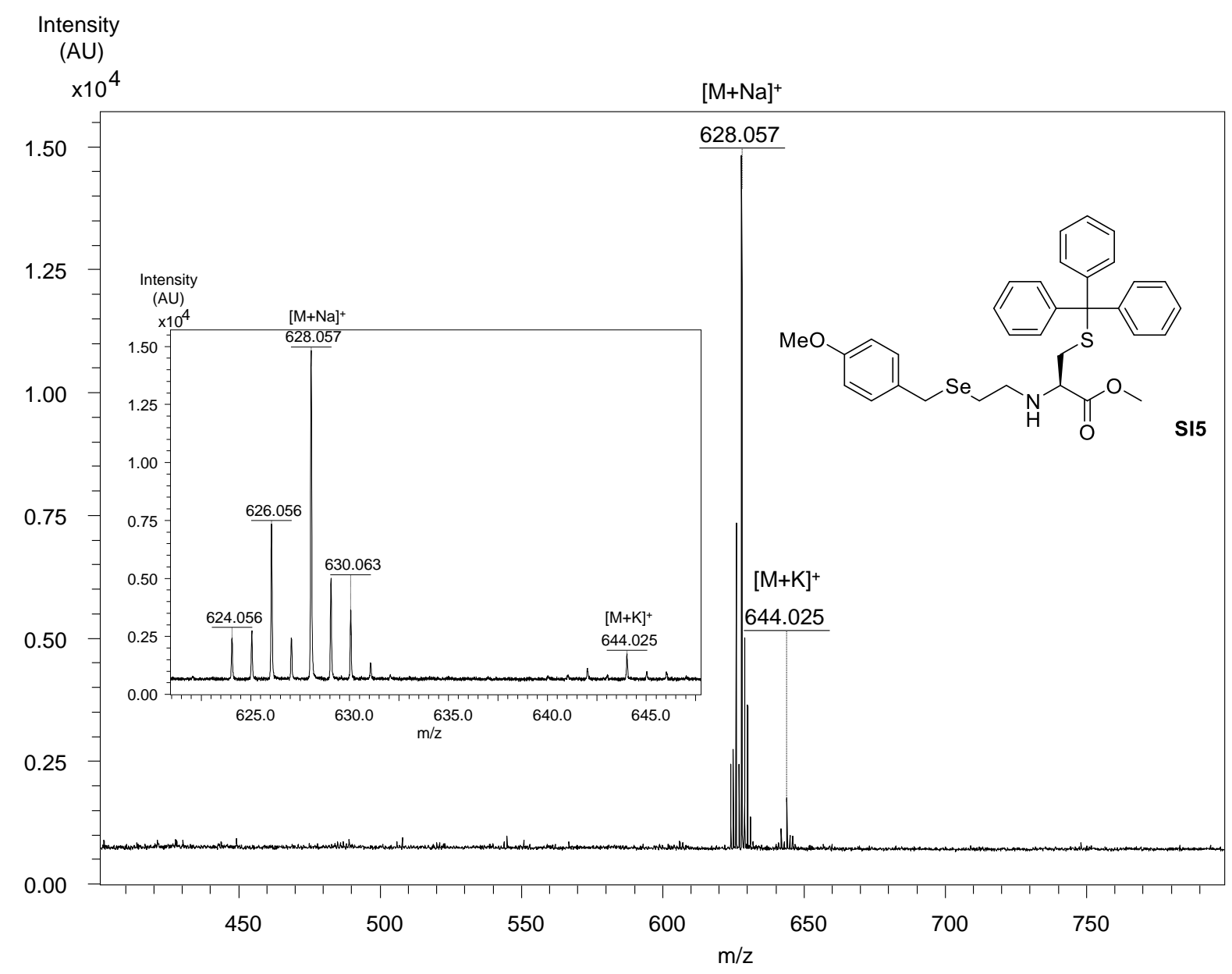

Figure S 6. MALDI-TOF analysis of $N$-[PMBSe-( $\left.\left.\mathrm{CH}_{2}\right)_{2}\right]-\mathrm{Cys}(\mathrm{Trt})-\mathrm{OMe}$ SI5. Matrix 2,5dihydroxybenzoic acid (DHB), positive detection mode, $[\mathrm{M}+\mathrm{Na}]^{+}$calcd. (monoisotopic) 628.14 , found 628.06 .

\subsection{Synthesis of $N$-Fmoc- $N$-[PMBSe-( $\left.\left.\mathrm{CH}_{2}\right)_{2}\right]-\mathrm{Cys}(\mathrm{Trt})-\mathrm{OMe}$}

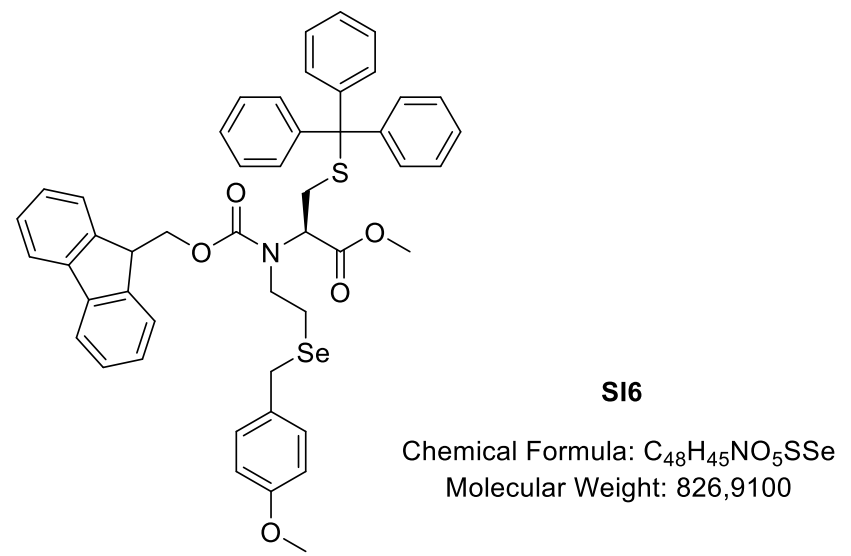

To a solution of $N$-[PMBSe $\left.\left(\mathrm{CH}_{2}\right)_{2}\right]$-Cys(Trt)-OMe SI5 (2.12 g, $\left.3.50 \mathrm{mmol}\right)$ in DCM (20 mL) were successively added DIEA $(0.64 \mathrm{~mL}, 3.67 \mathrm{mmol})$ and $\mathrm{FmocCl}(950 \mathrm{mg}, 3.67 \mathrm{mmol})$. The reaction mixture was stirred at $\mathrm{RT}$ for $24 \mathrm{~h}$ and was then diluted with DCM. The obtained 
organic layer was washed with water and was dried over $\mathrm{MgSO}_{4}$. After evaporation of the solvent under reduced pressure, purification of the crude by column chromatography (cyclohexane/EtOAc 7:3) provided the expected compound SI6 (1.87 g) as a white solid with $99 \%$ yield.

The titled compound was characterized by ${ }^{1} \mathrm{H}$ and ${ }^{13} \mathrm{C}$ NMR as a mixture of two conformers. $\mathrm{A} / \mathrm{B}=37: 63$. The ratio $\mathrm{A} / \mathrm{B}$ was calculated from the $\mathrm{CH}$ signals of the Fmoc protecting group.

${ }^{1} \mathbf{H}$ NMR $\left(300 \mathrm{MHz}, \mathrm{CDCl}_{3}\right) \delta 7.80\left(\mathrm{~d}, J=9.0 \mathrm{~Hz}, 0.64 \mathrm{H}, 2 \mathrm{H}_{\mathrm{A}}\right), 7.74\left(\mathrm{~d}, J=7.6 \mathrm{~Hz}, 2 \mathrm{H}_{\mathrm{B}}\right)$, 7.49-7.59 (m, $\left.2 \mathrm{H}_{\mathrm{A}}+2 \mathrm{H}_{\mathrm{B}}\right), 7.12-7.49\left(\mathrm{~m}, 21 \mathrm{H}_{\mathrm{A}}+19 \mathrm{H}_{\mathrm{B}}+\right.$ residual $\left.\mathrm{CDCl}_{3}\right), 7.08(\mathrm{~d}, J=8.3 \mathrm{~Hz}$, $\left.2 \mathrm{H}_{\mathrm{B}}\right), 6.89\left(\mathrm{~d}, J=8.3 \mathrm{~Hz}, 2 \mathrm{H}_{\mathrm{A}}\right), 6.71\left(\mathrm{~d}, J=8.4 \mathrm{~Hz}, 2 \mathrm{H}_{\mathrm{B}}\right), 4.34-4.60\left(\mathrm{~m}, 2 \mathrm{H}_{\mathrm{A}}+2 \mathrm{H}_{\mathrm{B}}\right), 4.20(\mathrm{t}$, $\left.J=5.9 \mathrm{~Hz}, \mathrm{H}_{\mathrm{B}}\right), 4.13\left(\mathrm{t}, J=5.2 \mathrm{~Hz}, \mathrm{H}_{\mathrm{A}}\right), 3.75\left(\mathrm{~s}, 3 \mathrm{H}_{\mathrm{A}}\right), 3.73\left(\mathrm{~s}, 2 \mathrm{H}_{\mathrm{A}}\right), 3.68\left(\mathrm{~s}, 3 \mathrm{H}_{\mathrm{B}}\right) \quad 3.40-3.63$ $\left(\mathrm{m}, \mathrm{H}_{\mathrm{A}}+5 \mathrm{H}_{\mathrm{B}}\right), 3.02-3.40\left(\mathrm{~m}, 4 \mathrm{H}_{\mathrm{A}}+2 \mathrm{H}_{\mathrm{B}}\right), 2.45-3.02\left(\mathrm{~m}, 3 \mathrm{H}_{\mathrm{A}}+3 \mathrm{H}_{\mathrm{B}}\right), 2.17-2.45\left(\mathrm{~m}, 2 \mathrm{H}_{\mathrm{A}}+\right.$ $\left.2 \mathrm{H}_{\mathrm{B}}\right) \mathrm{ppm} .{ }^{13} \mathrm{C} \mathbf{N M R}\left(75 \mathrm{MHz}, \mathrm{CDCl}_{3}\right) \delta 170.0(\mathrm{C}, \mathrm{B}), 169.7(\mathrm{C}, \mathrm{A}), 158.4(\mathrm{C}, \mathrm{A}+\mathrm{B}), 155.2$ $(\mathrm{C}, \mathrm{A}+\mathrm{B}), 144.5(\mathrm{C}, \mathrm{A}+\mathrm{B}), 143.7(\mathrm{C}, \mathrm{A}+\mathrm{B}), 141.4(\mathrm{C}, \mathrm{A}), 141.3(\mathrm{C}, \mathrm{B}), 131.3(\mathrm{C}, \mathrm{A}), 130.9$ $(\mathrm{C}, \mathrm{B}), 129.8(\mathrm{CH}, \mathrm{A}+\mathrm{B}), 129.6(\mathrm{CH}, \mathrm{A}+\mathrm{B}), 128.0(\mathrm{CH}, \mathrm{A}+\mathrm{B}), 127.7(\mathrm{CH}, \mathrm{A}+\mathrm{B}), 127.1$ $(\mathrm{CH}, \mathrm{A}+\mathrm{B}), 126.8(\mathrm{CH}, \mathrm{A}+\mathrm{B}), 124.3-125.3(\mathrm{~m}, \mathrm{CH}, \mathrm{A}+\mathrm{B}), 120.0(\mathrm{CH}, \mathrm{A}+\mathrm{B}), 113.9(\mathrm{~A}+$ B), $67.2\left(\mathrm{CH}_{2}, \mathrm{~A}\right), 67.0\left(\mathrm{CH}_{2}, \mathrm{~B}\right), 60.6(\mathrm{CH}, \mathrm{B}), 59.8(\mathrm{CH}, \mathrm{A}), 55.1\left(\mathrm{CH}_{3}, \mathrm{~A}+\mathrm{B}\right), 52.3\left(\mathrm{CH}_{3}\right.$, B), $52.2\left(\mathrm{CH}_{3}, \mathrm{~A}\right), 49.6\left(\mathrm{CH}_{2}, \mathrm{~A}\right), 49.2\left(\mathrm{CH}_{2}, \mathrm{~B}\right), 47.2(\mathrm{CH}, \mathrm{B}), 47.1(\mathrm{CH}, \mathrm{A}), 31.5\left(\mathrm{CH}_{2}, \mathrm{~A}\right)$, $31.2\left(\mathrm{CH}_{2}, \mathrm{~B}\right), 26.5\left(\mathrm{CH}_{2}, \mathrm{~A}+\mathrm{B}\right), 21.3\left(\mathrm{CH}_{2}, \mathrm{~B}\right), 20.8\left(\mathrm{CH}_{2}, \mathrm{~A}\right) \mathrm{ppm}$. IR (ATR, cm $\left.{ }^{-1}\right) 3054$, 2949, 1742, 1699, 1509, 1445, 1283, 1245, 741, 701. HRMS $\left(\mathrm{ES}^{+}\right)$: Calcd. for $\mathrm{C}_{48} \mathrm{H}_{45} \mathrm{NO}_{5} \mathrm{SNaSe}: 850.2081$, found: 850.2089 . [ $\left.\boldsymbol{\alpha}\right]_{\mathbf{D}^{20}}{ }^{\mathbf{2}}$ (c 1.0, $\left.\mathrm{CHCl}_{3}\right)$ : -38 。.

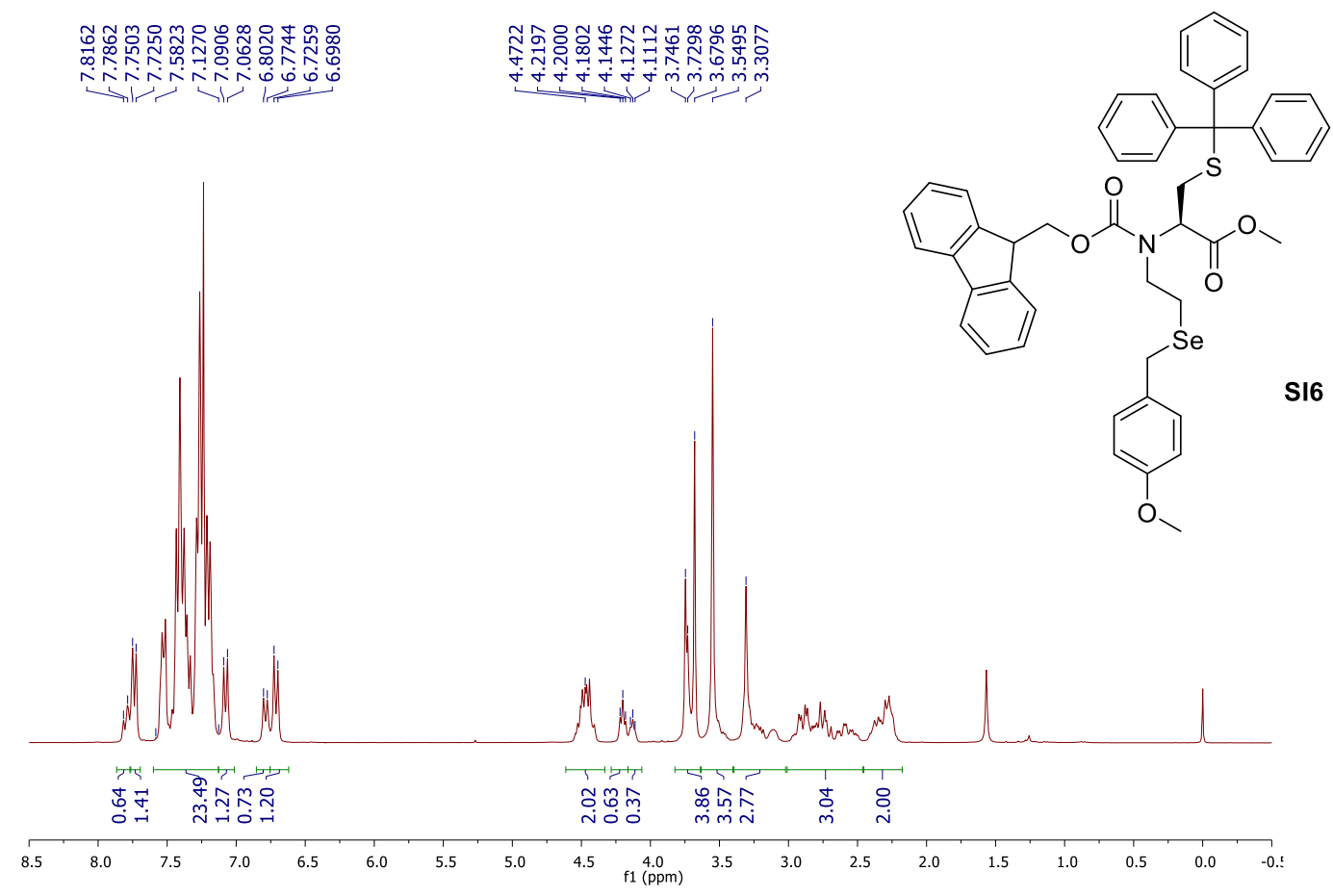

Figure S 7. ${ }^{1} \mathrm{H}$ NMR (300 MHz) spectrum of $\boldsymbol{N}$-Fmoc- $\boldsymbol{N}$-[PMBSe-(CH2) $]$-Cys(Trt)-OMe SI6 $\left(\mathrm{CDCl}_{3}, 293 \mathrm{~K}\right)$. 


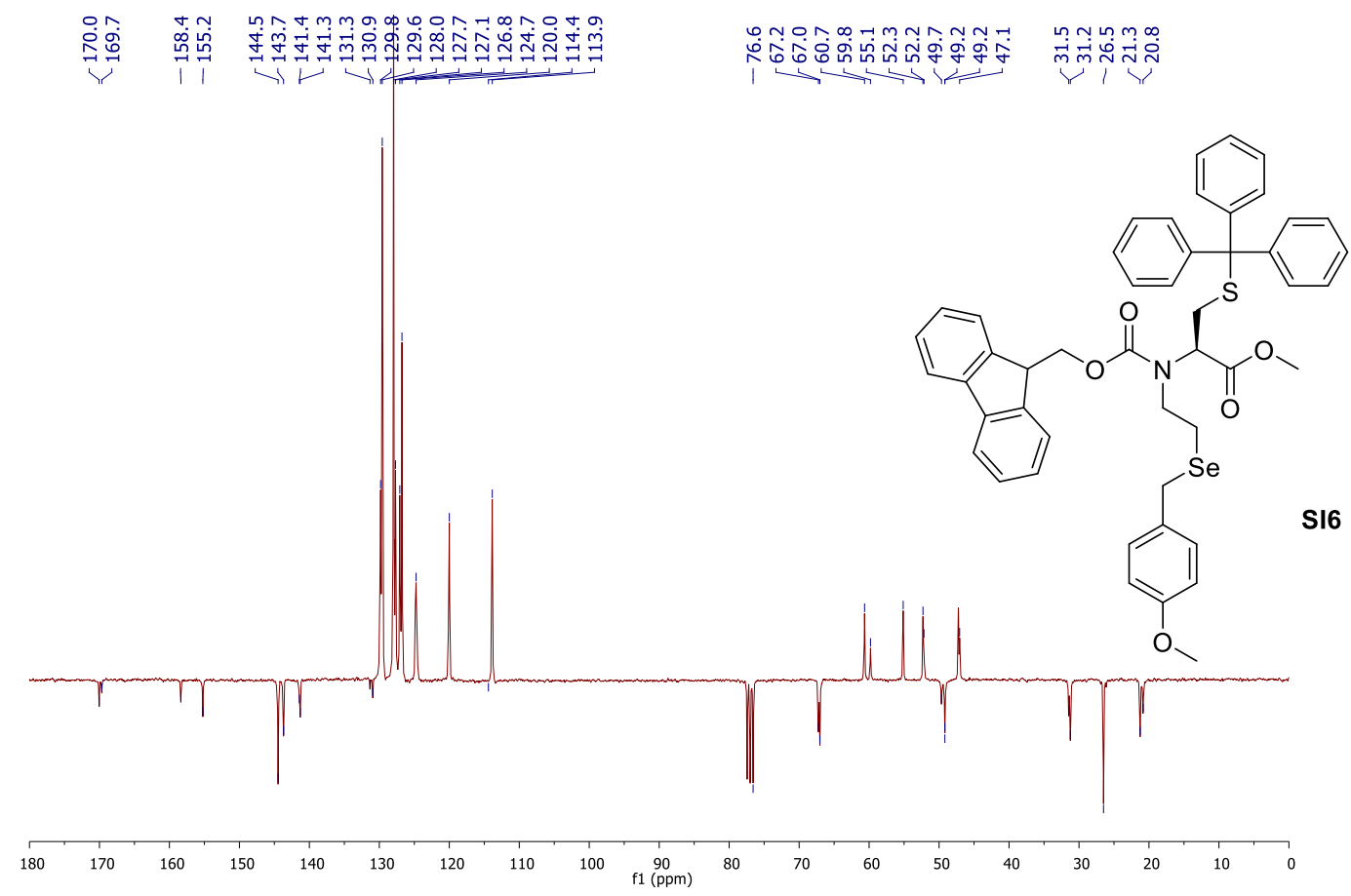

Figure S 8. ${ }^{13} \mathrm{C}$ JMOD NMR (75 MHz) spectrum of $\boldsymbol{N}$-Fmoc- $N$-[PMBSe-(CH2)2]-Cys(Trt)OMe SI6 $\left(\mathrm{CDCl}_{3}, 293 \mathrm{~K}\right)$.

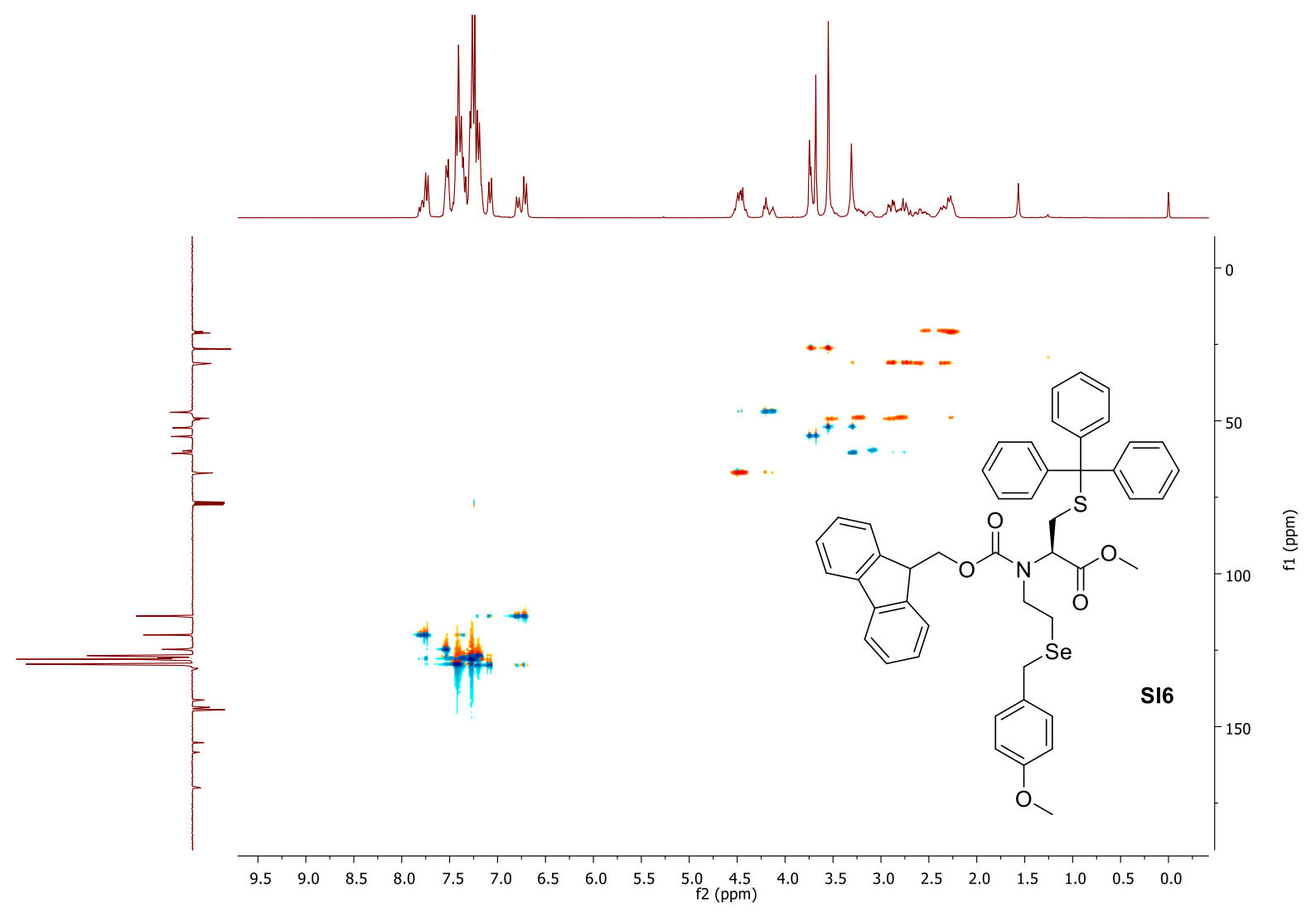

Figure S 9. ${ }^{1} \mathrm{H}^{13} \mathrm{C}$ HSQC spectrum of $N$-Fmoc- $N$-[PMBSe-(CH2)2]-Cys(Trt)-OMe SI6 $\left(\mathrm{CDCl}_{3}, 293 \mathrm{~K}\right)$. 


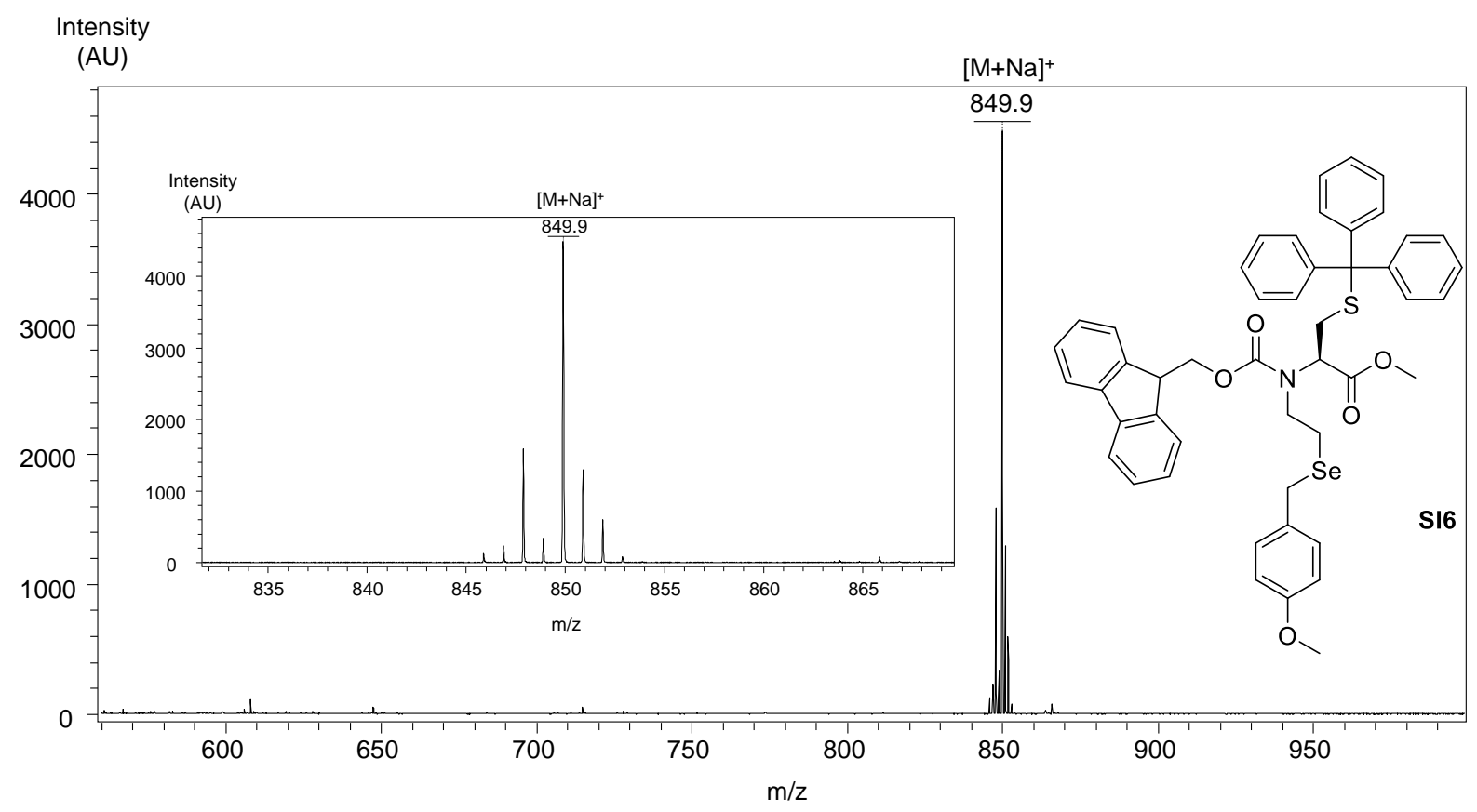

Figure S 10. MALDI-TOF analysis of $N$-Fmoc- $N$-[PMBSe- $\left.\left(\mathrm{CH}_{2}\right)_{2}\right]-\mathrm{Cys}(\mathrm{Trt})-\mathrm{OMe}$ SI6. Matrix 2,5-dihydroxybenzoic acid (DHB), positive detection mode, $[\mathrm{M}+\mathrm{Na}]^{+}$calcd. (monoisotopic) 850.22, found 849.9.

\subsection{Synthesis of Fmoc-SetCys-OMe}

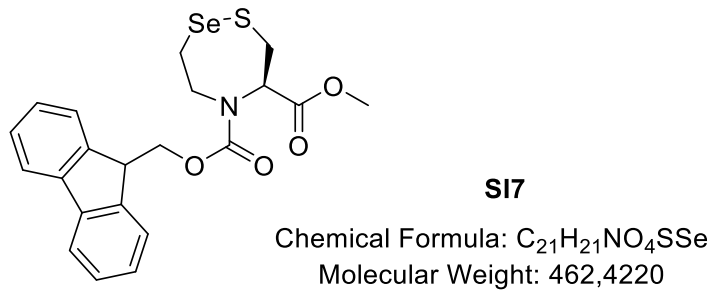

To a solution of $\boldsymbol{N}$-Fmoc- $\boldsymbol{N}$-[PMBSe-(CH2)2]-Cys(Trt)-OMe SI6 (1.67 g, 2.02) in DCM (50 $\mathrm{mL}$ ) was added $\mathrm{NaHCO}_{3}(508 \mathrm{mg}, 6.06 \mathrm{mmol})$. The mixture was cooled to $0{ }^{\circ} \mathrm{C}$ and $\mathrm{I}_{2}(1.54 \mathrm{~g}$, $6.06 \mathrm{mmol})$ dissolved in DCM $(50 \mathrm{~mL})$ was added dropwise. The reaction mixture was stirred at $\mathrm{RT}$ for $30 \mathrm{~min}$ and was washed $1 \mathrm{M} \mathrm{Na}_{2} \mathrm{~S}_{2} \mathrm{O}_{3}(50 \mathrm{~mL})$ to remove the excess of $\mathrm{I}_{2}$. The organic layer was separated and the aqueous layer was extracted with DCM $(2 \times 50 \mathrm{~mL})$. Note that the separation between the aqueous and organic layers was improved by addition of brine $(50 \mathrm{~mL})$. All the organic layers were then combined, washed with brine $(50 \mathrm{~mL})$ and dried over $\mathrm{MgSO}_{4}$. After evaporation of the solvent, purification of the crude by column chromatography (cyclohexane/EtOAc 8:2) provided the expected compound SI7 (780 mg) as a glass with 83\% yield. 
The titled compound was characterized by ${ }^{1} \mathrm{H}$ and ${ }^{13} \mathrm{C}$ NMR as a mixture of two main conformers. $\mathrm{A} / \mathrm{B}=45: 55$. The ratio $\mathrm{A} / \mathrm{B}$ was calculated from the aromatic $\mathrm{CH}$ signals of the Fmoc protecting group. Due to overlapping signals, no clear description of the ${ }^{1} \mathrm{H}$ NMR spectrum can be provided.

${ }^{13} \mathrm{C}$ NMR $\left(75 \mathrm{MHz}, \mathrm{CDCl}_{3}\right) \delta 170.0(\mathrm{C}), 169.7(\mathrm{C}), 155.6(\mathrm{C}), 155.2(\mathrm{C}), 143.90(\mathrm{C}), 143.88$ (C), $143.84(\mathrm{C}), 143.6(\mathrm{C}), 141.6(\mathrm{C}), 141.53(\mathrm{C}), 141.51(\mathrm{C}), 127.91(\mathrm{CH}), 127.88(\mathrm{CH})$, $127.86(\mathrm{CH}), 127.7(\mathrm{CH}), 127.34(\mathrm{CH}), 127.30(\mathrm{CH}), 127.25(\mathrm{CH}), 127.1(\mathrm{CH}), 124.8(\mathrm{CH})$, $124.4(\mathrm{CH}), 124.3(\mathrm{CH}), 67.1\left(\mathrm{CH}_{2}\right), 66.8\left(\mathrm{CH}_{2}\right), 62.7(\mathrm{CH}), 52.7\left(\mathrm{CH}_{3}\right), 52.4\left(\mathrm{CH}_{3}\right), 49.8$ $\left(\mathrm{CH}_{2}\right), 47.4(\mathrm{CH}), 47.2(\mathrm{CH}), 38.6\left(\mathrm{CH}_{2}\right), 29.3\left(\mathrm{CH}_{2}\right), 29.1\left(\mathrm{CH}_{2}\right) \mathrm{ppm}$. IR $\left(\mathrm{ATR}, \mathrm{cm}^{-1}\right) 2948$, 1740, 1697, 1474, 1449, 1417, 1278, 738. HRMS $\left(\mathrm{ES}^{+}\right)$Calcd. for $\mathrm{C}_{21} \mathrm{H}_{21} \mathrm{NO}_{4} \mathrm{NaSSe}$ : 486.0254, found: 486.0246. [a] $\mathbf{D}^{\mathbf{2 0}}$ (c 1.0, $\left.\mathrm{CHCl}_{3}\right):-29^{\circ}$.

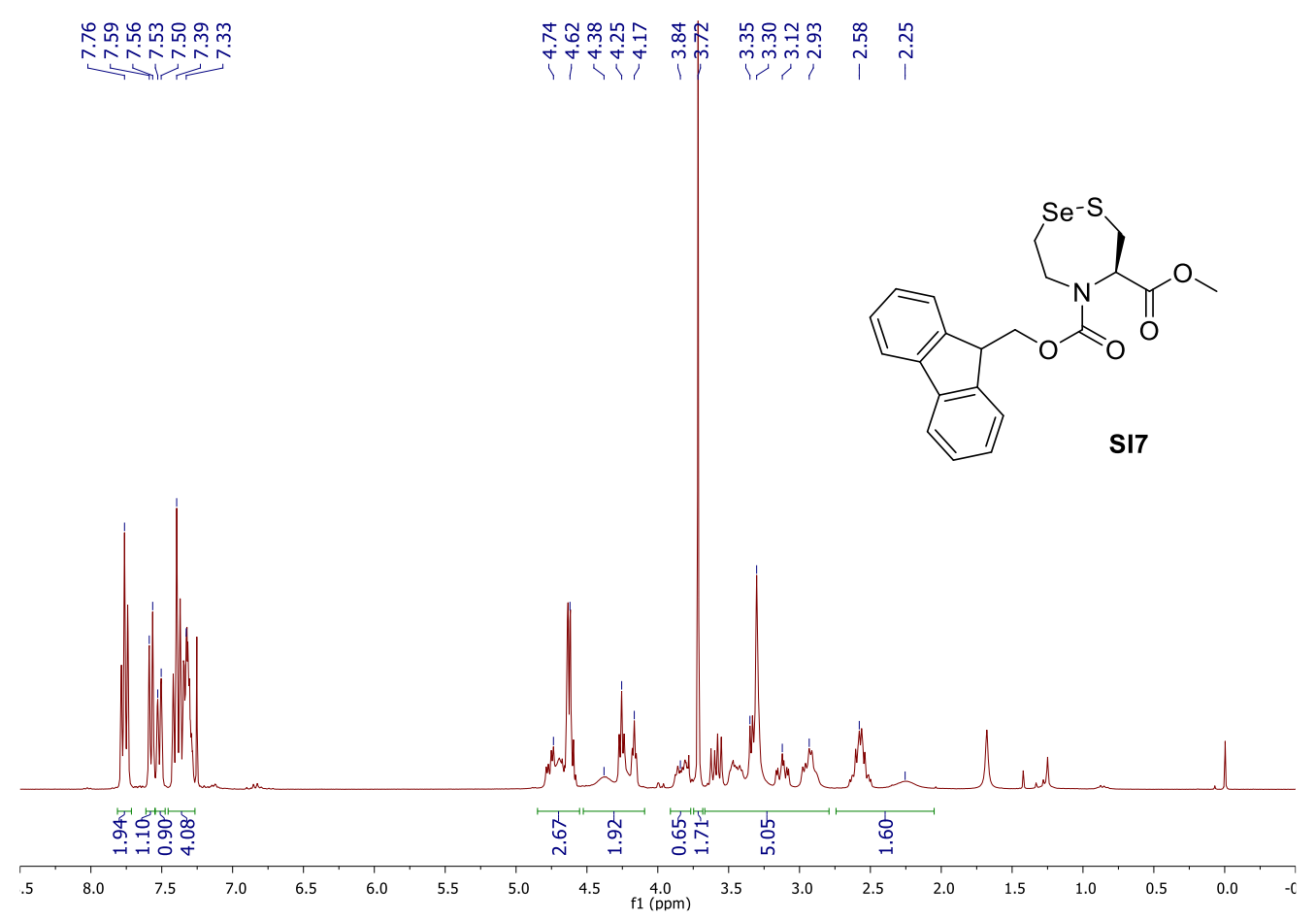

Figure S 11. ${ }^{1} \mathrm{H}$ NMR (300 MHz) spectrum of Fmoc-SetCys-OMe SI7 (CDCl 3 , $\left.293 \mathrm{~K}\right)$. 

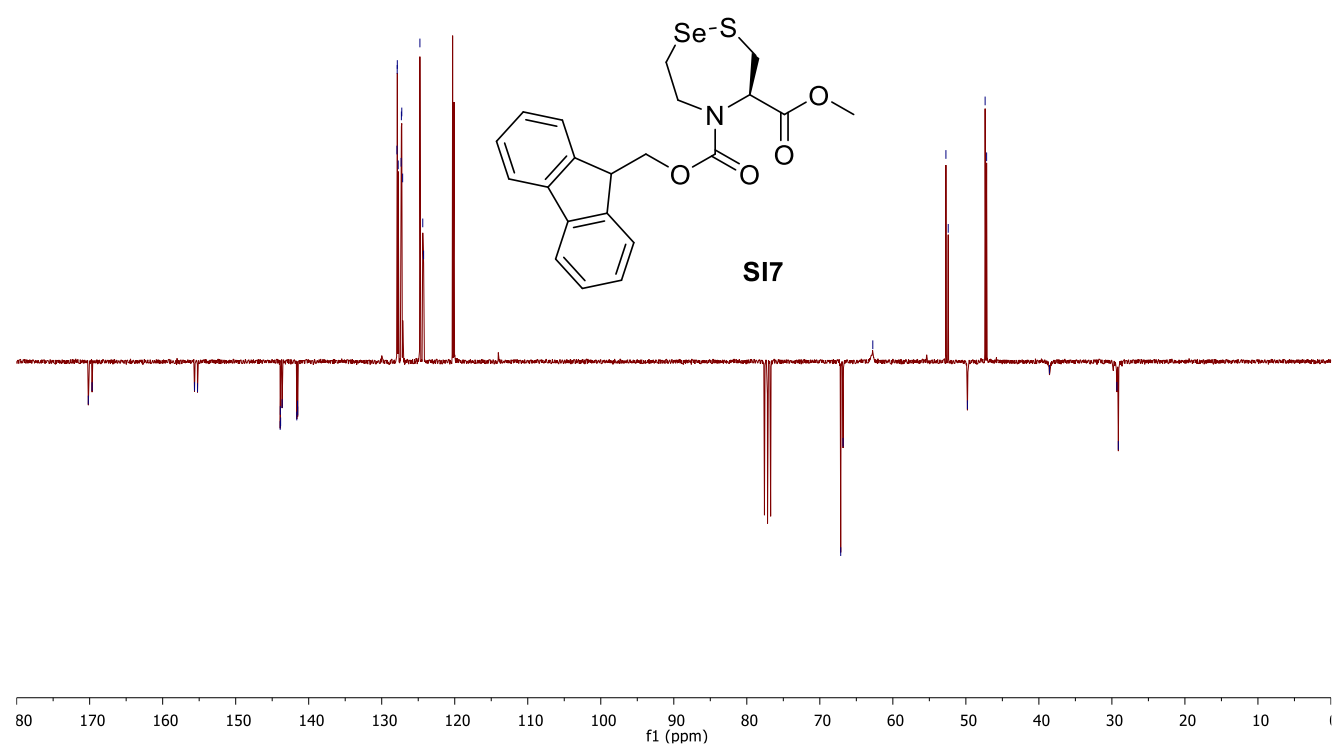

Figure S 12. ${ }^{13} \mathrm{C} \mathrm{JMOD} \mathrm{NMR} \mathrm{(75} \mathrm{MHz)} \mathrm{spectrum} \mathrm{of} \mathrm{Fmoc-SetCys-OMe} \mathrm{SI7}\left(\mathrm{CDCl}_{3}, 293 \mathrm{~K}\right)$.

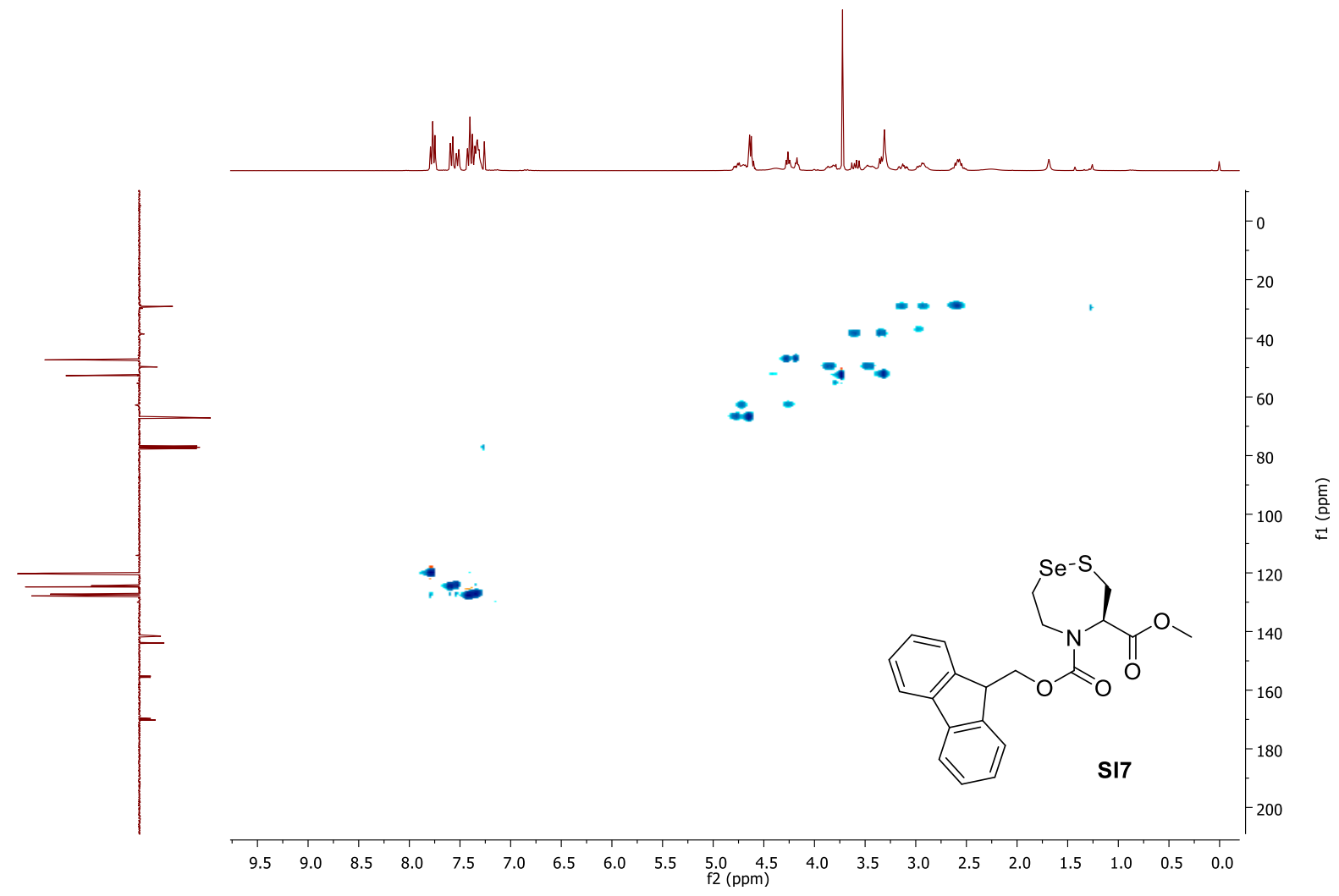

Figure S 13. ${ }^{1} \mathrm{H}-{ }^{13} \mathrm{C}$ HSQC spectrum of Fmoc-SetCys-OMe SI7 $\left(\mathrm{CDCl}_{3}, 293 \mathrm{~K}\right)$. 


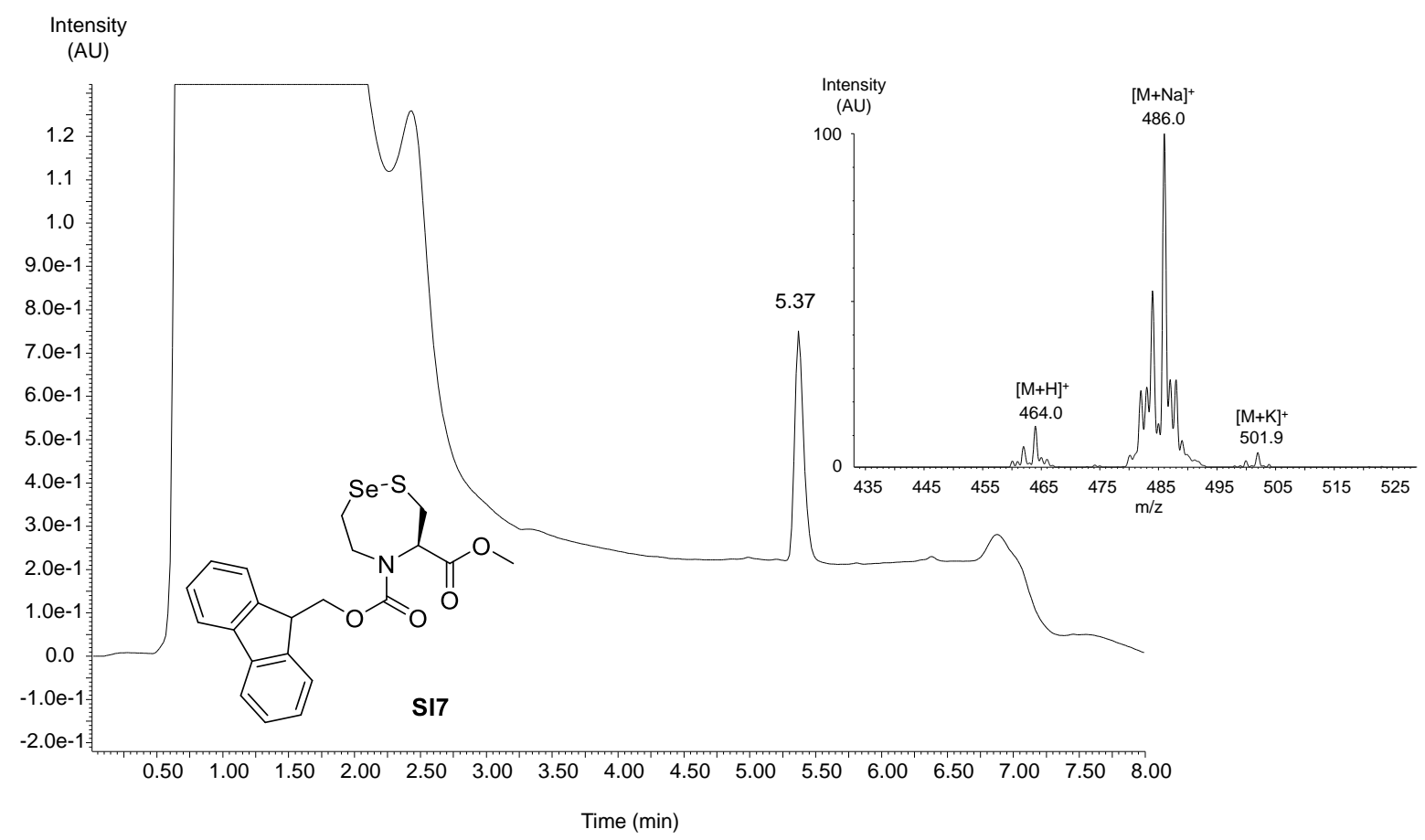

Figure S 14. LC-MS analysis of Fmoc-SetCys-OMe SI7. LC trace: eluent C $0.10 \%$ formic acid in water, eluent $\mathrm{D} 0.10 \%$ formic acid in $\mathrm{CH}_{3} \mathrm{CN} /$ water: $4 / 1$ by vol. $\mathrm{C} 18$ column, gradient $0-100 \% \mathrm{D}$ in $4 \mathrm{~min}, 50{ }^{\circ} \mathrm{C}, 0.5 \mathrm{~mL} / \mathrm{min}$, UV detection. MS trace: $[\mathrm{M}+\mathrm{H}]^{+} \mathrm{m} / \mathrm{z}$ calcd. (monoisotopic mass) 464.04, found 464.0; $[\mathrm{M}+\mathrm{Na}]^{+} \mathrm{m} / \mathrm{z}$ calcd. (monoisotopic mass) 486.04, found 486.0 .

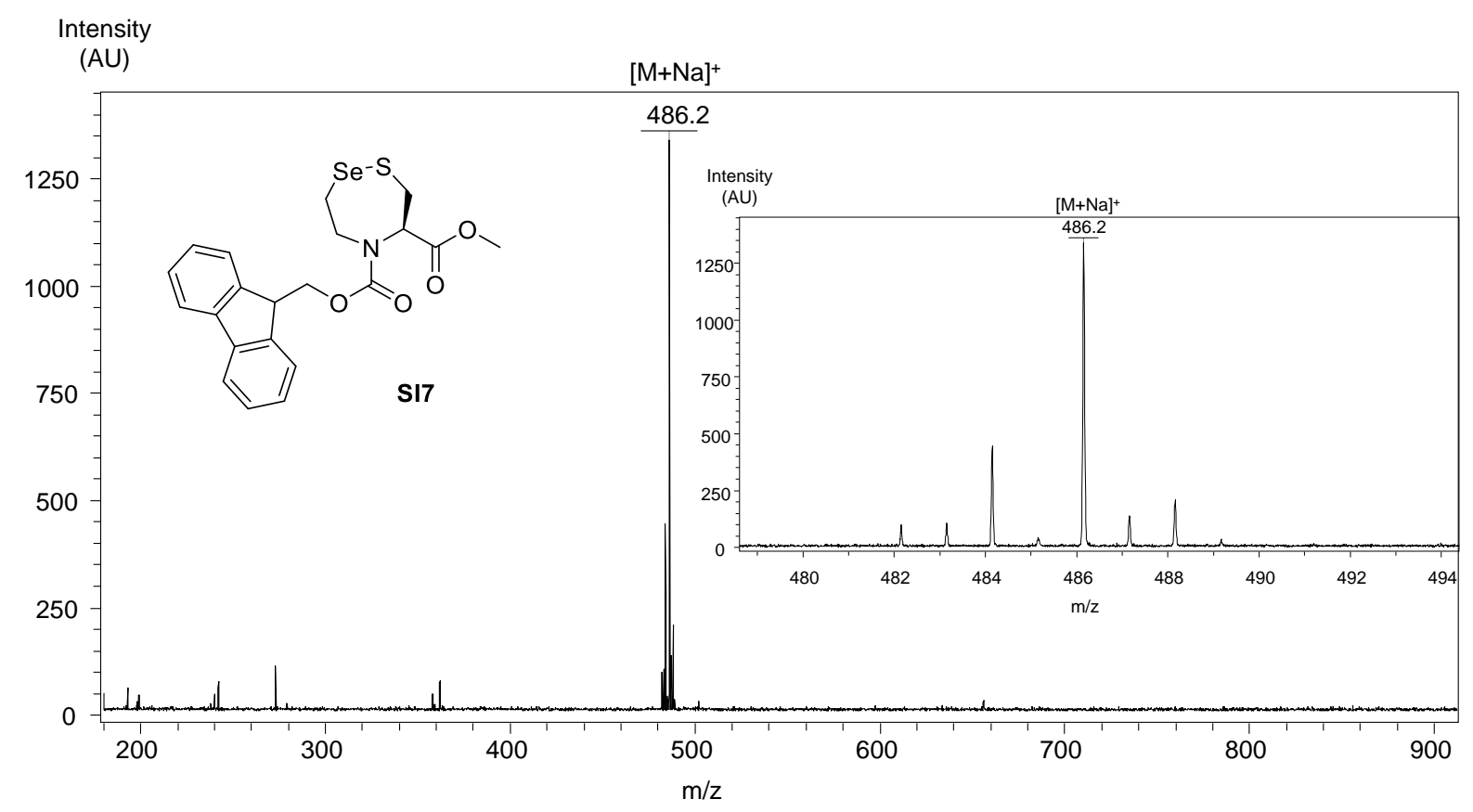

Figure S 15. MALDI-TOF analysis of Fmoc-SetCys-OMe SI7. Matrix 2,5-dihydroxybenzoic acid (DHB), positive detection mode, $[\mathrm{M}+\mathrm{Na}]^{+}$calcd. (monoisotopic) 486.04, found 486.2. 


\subsection{Synthesis of Fmoc-SetCys-OH}

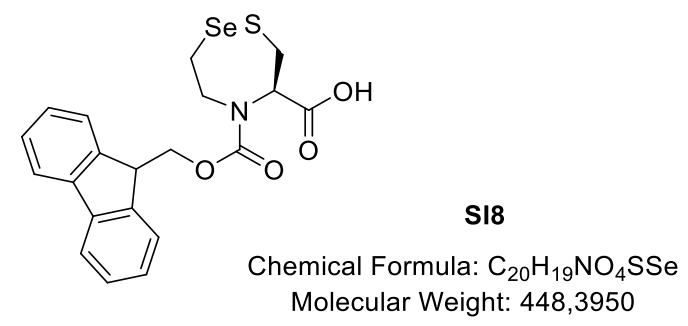

To a solution of Fmoc-SetCys-OMe SI7 $(411 \mathrm{mg})$ in dioxane $(19 \mathrm{~mL})$ was added $5 \mathrm{M} \mathrm{HCl}(9$ $\mathrm{mL}$ ) and the mixture was refluxed for $6 \mathrm{~h}$. The reaction mixture was cooled to RT and 5\% $\mathrm{K}_{2} \mathrm{CO}_{3}(200 \mathrm{~mL})$ was added to raise the $\mathrm{pH}$ above 9. The aqueous layer was then washed with $\mathrm{E}_{2} \mathrm{O}(2 \times 100 \mathrm{~mL})$, acidified with concentrated $\mathrm{HCl}$ until $\mathrm{pH} \approx 2-3$ and extracted with DCM (3 $\times 100 \mathrm{~mL}$ ). The organic layers $(\mathrm{DCM})$ were combined and dried over $\mathrm{MgSO}_{4}$. Evaporation of the solvent under reduced provided the expected amino acid SI8 (345 mg, 86\%) as a white solid which was used in the next step without further purification.

The titled compound was characterized by ${ }^{1} \mathrm{H}$ and ${ }^{13} \mathrm{C}$ NMR as a mixture of two main conformers. Due to overlapping signals, no clear description of the ${ }^{1} \mathrm{H}$ NMR spectrum can be provided. IR (ATR, cm ${ }^{-1}$ ) 3000 (broad signal), 1699 (broad), 1476, 1450, 1419, 1286, 1190,

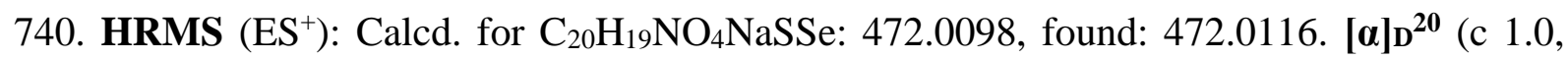
$\left.\mathrm{CHCl}_{3}\right):-32^{\circ}$.

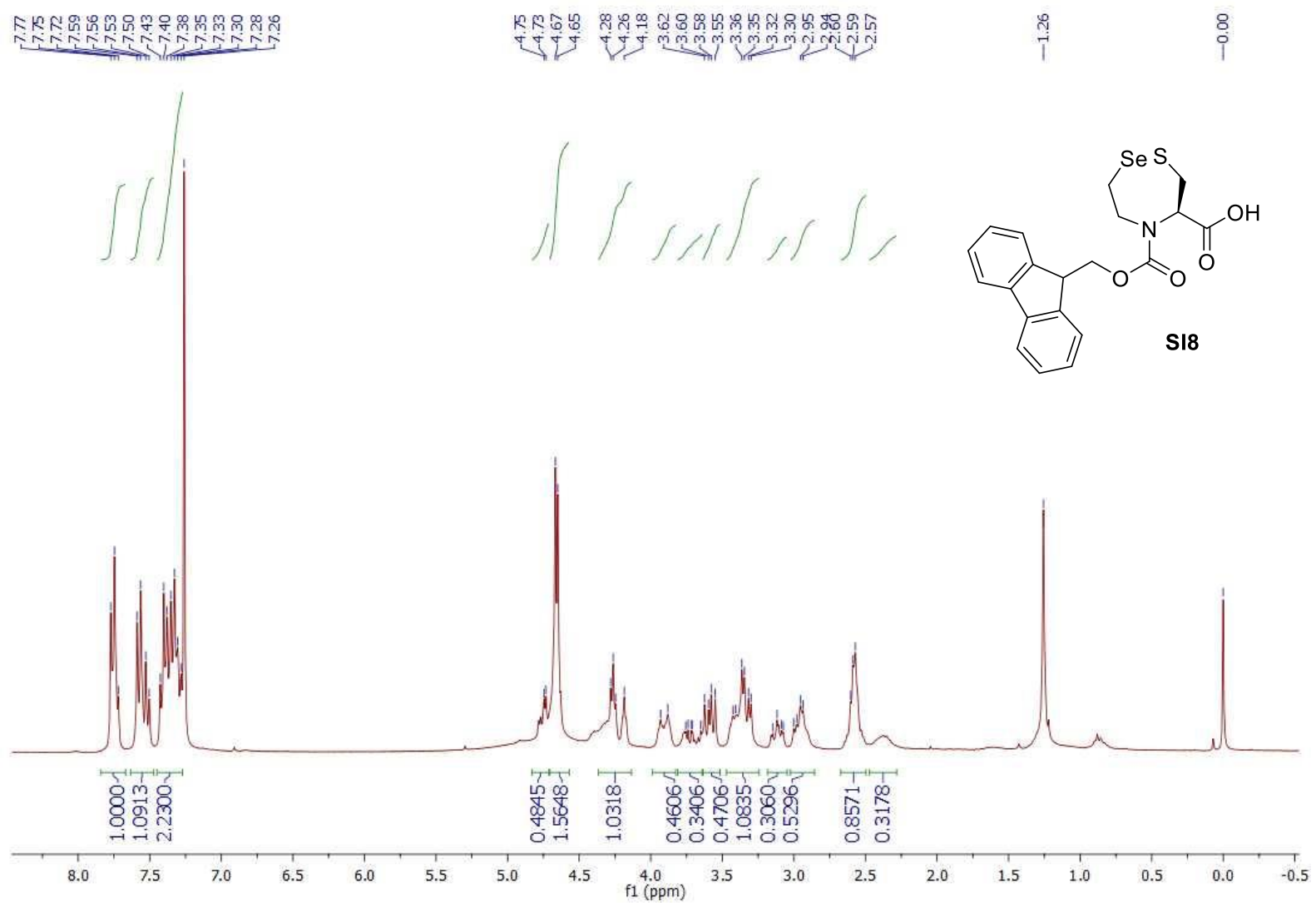

Figure S 16. ${ }^{1} \mathrm{H}$ NMR (300 MHz) spectrum of Fmoc-SetCys-OH SI8 ( $\left.\mathrm{CDCl}_{3}, 293 \mathrm{~K}\right)$. 


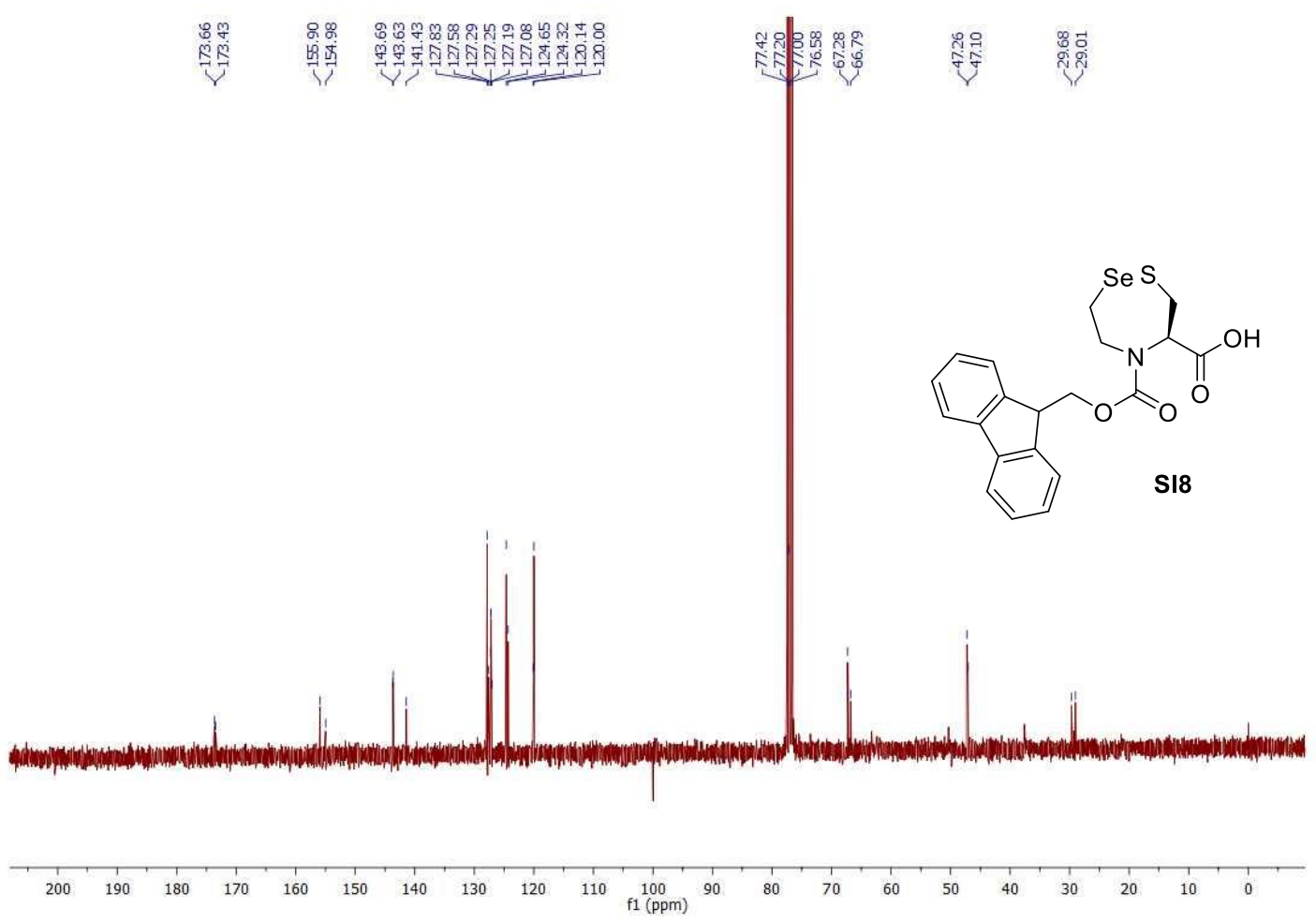

Figure S 17. ${ }^{13} \mathrm{C}$ NMR (75 MHz) spectrum of Fmoc-SetCys-OH SI8 $\left(\mathrm{CDCl}_{3}, 293 \mathrm{~K}\right)$.

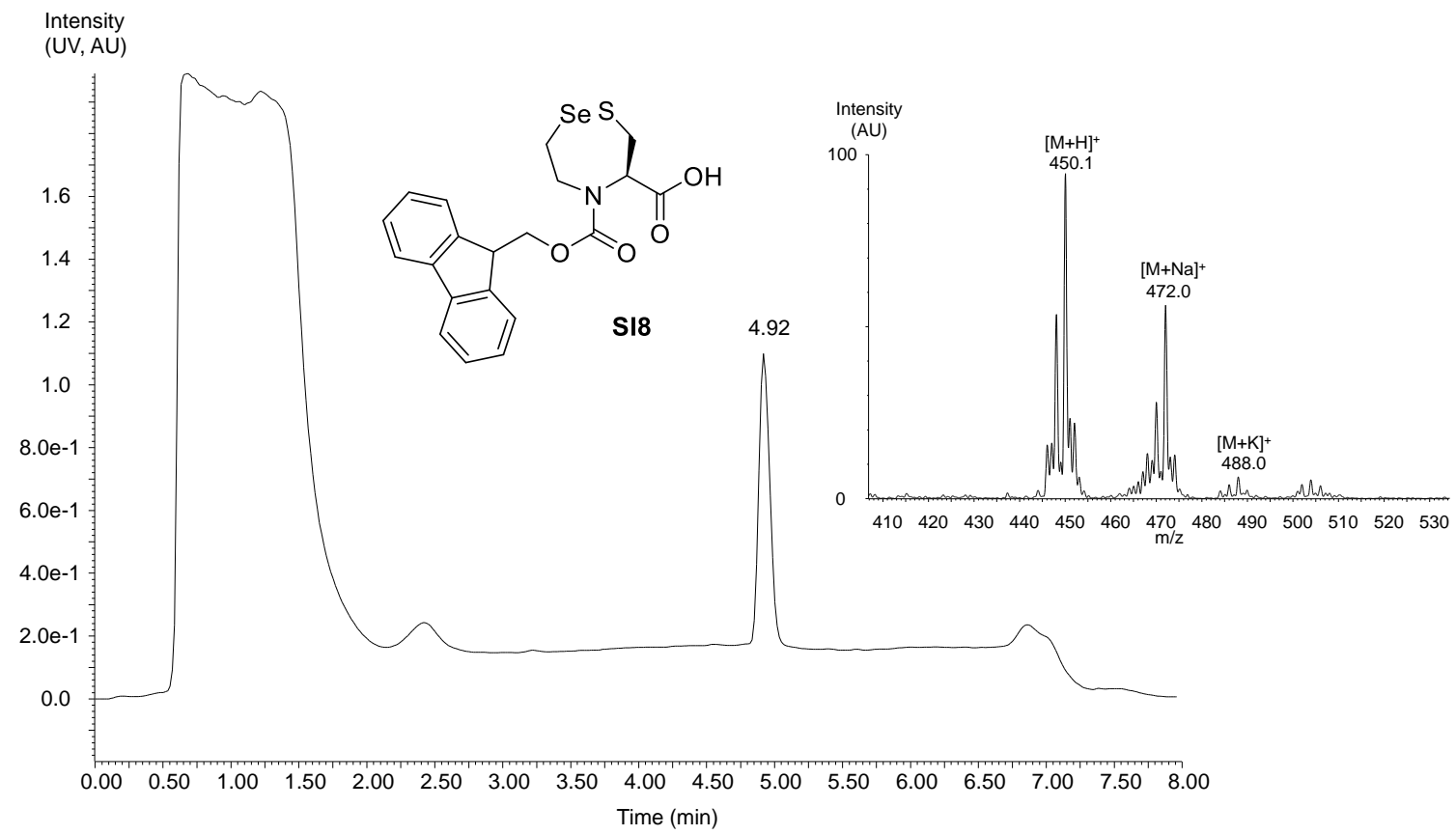

Figure S 18. LC-MS analysis of Fmoc-SetCys-OH SI8. LC trace: eluent C 0.10\% formic acid in water, eluent $\mathrm{D} 0.10 \%$ formic acid in $\mathrm{CH}_{3} \mathrm{CN} /$ water: $4 / 1$ by vol. $\mathrm{C} 18$ column, gradient 0 $100 \% \mathrm{D}$ in $4 \mathrm{~min}, 50{ }^{\circ} \mathrm{C}, 0.5 \mathrm{~mL} / \mathrm{min}$, UV detection. MS trace: $[\mathrm{M}+\mathrm{H}]^{+} \mathrm{m} / \mathrm{z}$ calcd. (monoisotopic mass) 450.02, found 450.1; $[\mathrm{M}+\mathrm{Na}]^{+} \mathrm{m} / \mathrm{z}$ calcd. (monoisotopic mass) 472.02, found 472.0 . 


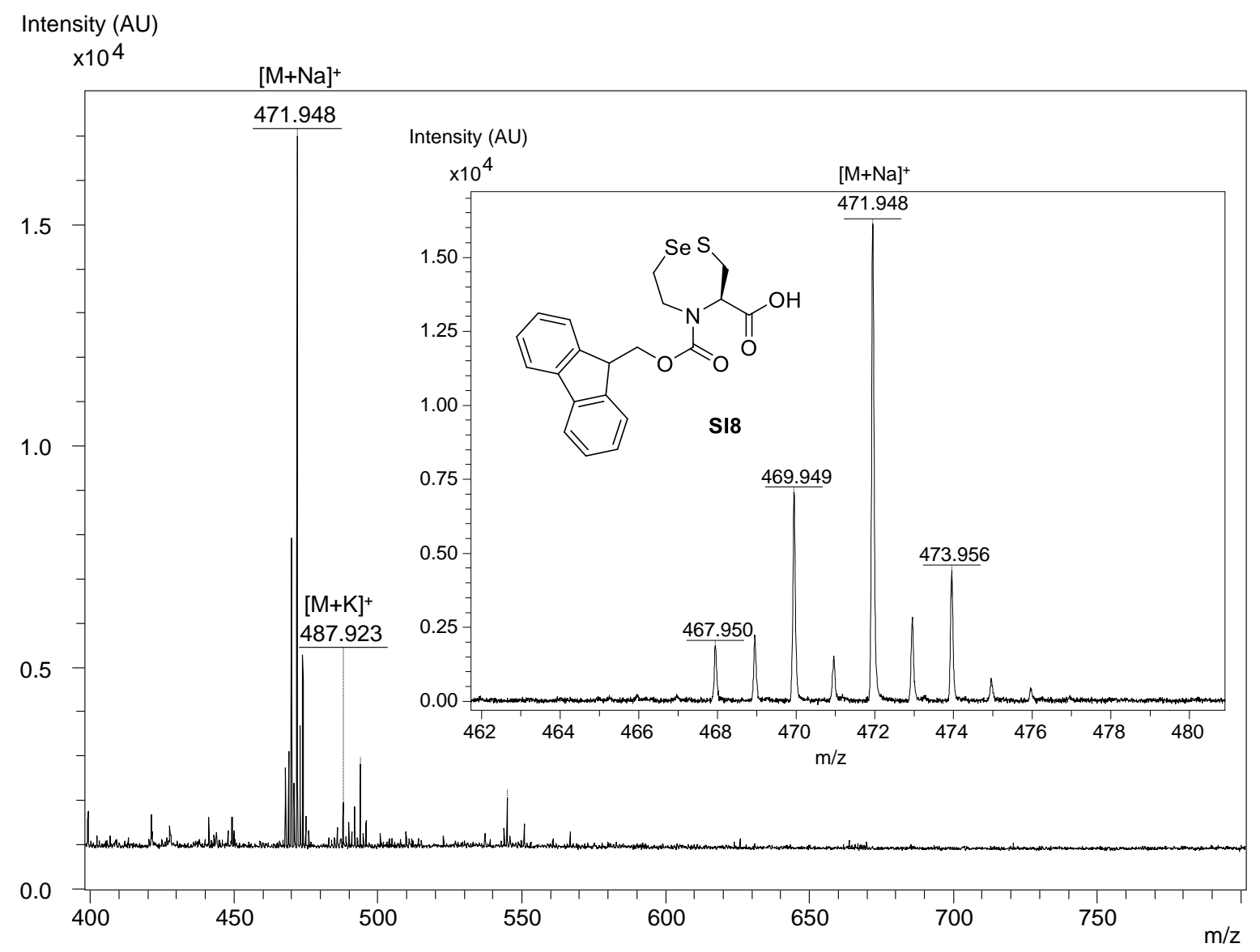

Figure S 19. MALDI-TOF analysis of Fmoc-SetCys-OH SI8. Matrix 2,5-dihydroxybenzoic acid (DHB), positive detection mode, $[\mathrm{M}+\mathrm{Na}]^{+}$calcd. (monoisotopic) 472.01, found 471.95. 


\section{Peptide synthesis}

CILKEPVHGV-NH2 has already been synthesized and characterized elsewhere. ${ }^{\text {Erreur }}$ ! Signet non d éfini.

\subsection{General procedures}

Peptide amides were synthesized on a NovaSyn TGR resin $(0.25 \mathrm{mmol} / \mathrm{g})$ using standard Fmoc chemistry and an automated peptide synthesizer (Figure S 20). Note that the Fmoc-SetCys-OH amino acid was manually introduced in the peptide sequence.

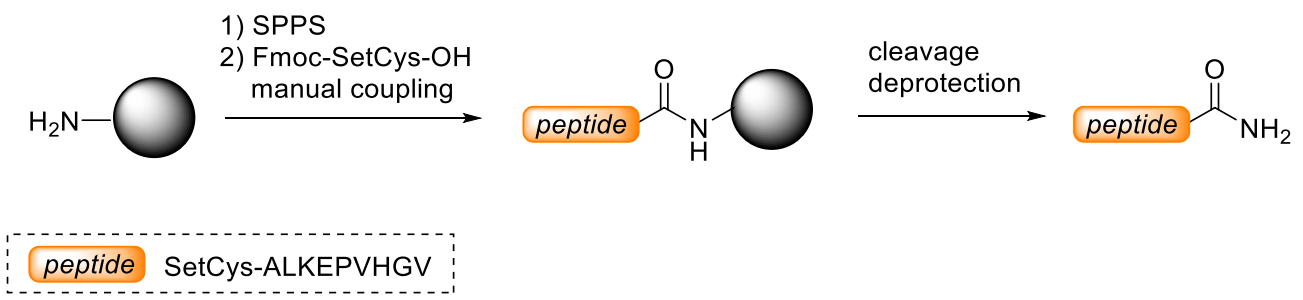

Figure S 20. General approach for the preparation of peptide amides.

SEA $^{\text {off }}$ peptides were synthesized on SEA PS resin $(0.16 \mathrm{mmol} / \mathrm{g})$ using standard Fmoc chemistry and an automated peptide synthesizer (Figure S 21). The SEA ${ }^{\text {on }}$ peptide recovered after acidic cleavage was converted into the corresponding SEA ${ }^{\text {off }}$ peptide by oxidation with iodine. The same intermediate provided the MPA thioester via a SEA-MPA exchange reaction. Detailed procedures to prepare these peptides are available in previous papers. ${ }^{3,4,5}$ Note that first amino acids coupled to the resin, biotinylated lysine residues and the Fmoc-SetCys-OH amino acids were introduced manually in the peptide sequence. 


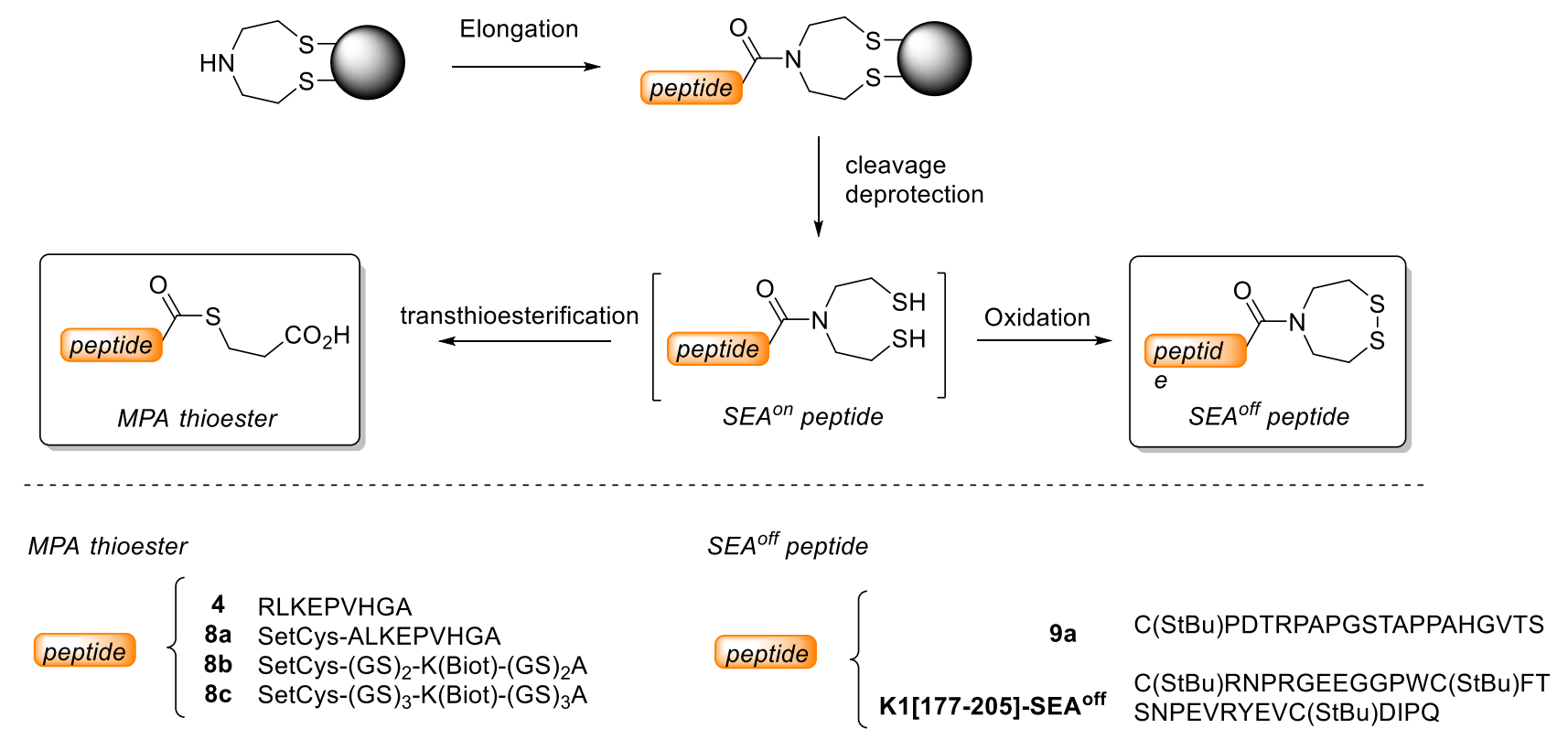

Figure S 21. Synthesis of SEA ${ }^{\text {off }}$ peptides and MPA thioesters.

\section{Coupling of the first amino acid on SEA PS resin}

The first amino acid (10 eq) was coupled to SEA PS resin (1 eq) using HATU (10 eq)/DIEA (20 eq) activation in the minimal volume of DMF. The amino acid was preactivated for $2 \mathrm{~min}$ and was then added to the resin swelled in the minimal volume of DMF. The beads were agitated at RT for $1.5 \mathrm{~h}$ and the resin was then washed with DMF $(3 \times 2 \mathrm{~min})$ and drained. The absence of unreacted secondary amino groups was checked using chloranyl colorimetric assay. A capping step was then performed using $\mathrm{Ac}_{2} \mathrm{O} / \mathrm{DIEA} / \mathrm{DMF}$ 10:5:85 $(2 \times 5 \mathrm{~min})$ and the resin was washed with DMF $(3 \times 2 \mathrm{~min})$.

\section{Automated peptide elongation}

Peptide elongation was performed using standard Fmoc chemistry on an automated peptide synthesizer without microwaves. Couplings were performed using 4-fold molar excess of each Fmoc L-amino acid, 3.6-fold molar excess of HBTU, and 8-fold molar excess of DIEA. A capping step was performed with $\mathrm{Ac}_{2} \mathrm{O} / \mathrm{DIEA} / \mathrm{DMF}$ 10:5:85 before Fmoc deprotection (piperidine/DMF 80:20).

\section{Coupling of the biotinylated lysine}

To a solution of HATU (190 mg, $0.5 \mathrm{mmol}, 5 \mathrm{eq})$ in DMF ( $3 \mathrm{~mL})$ were successively added FmocLys(Biot)OH (300 mg, $0.64 \mathrm{mmol}, 6.4 \mathrm{eq})$ and DIEA (174 $\mu \mathrm{L}, 1.0 \mathrm{mmol}, 10 \mathrm{eq})$ and the mixture was stirred at RT for $2 \mathrm{~min}$. The preactivated amino acid was then added to the resin ( $0.1 \mathrm{mmol}, 1 \mathrm{eq})$ swelled in a minimal volume of DMF. The beads were agitated at RT for 1.5 $\mathrm{h}$ and were then washed with DMF $(3 \times 2 \mathrm{~min})$. A capping step was then performed using $\mathrm{Ac}_{2} \mathrm{O} / \mathrm{DIEA} / \mathrm{DMF}$ 10:5:85 (2 $\times 5$ min $)$. Finally, the resin was washed again with DMF $(3 \times 2$ $\min )$. 


\section{Coupling of the Fmoc-SetCys-OH amino acid}

To a solution of Fmoc-SetCys-OH (44.8 mg, $0.10 \mathrm{mmol}, 2 \mathrm{eq})$ in DMF (2.5 mL) were successively added HOBt $(15.3 \mathrm{mg}, 0.10 \mathrm{mmol}, 2 \mathrm{eq})$ and DIC (15.6 $\mu \mathrm{L}, 0.10 \mathrm{mmol}, 2 \mathrm{eq})$ and the mixture was stirred at RT for $2 \mathrm{~min}$. The preactivated amino acid was then added to the resin $(0.1 \mathrm{mmol}, 1 \mathrm{eq})$ swelled in a minimal volume of DMF. The beads were agitated at RT for $3 \mathrm{~h}$ and washed with DMF ( $3 \times 2 \mathrm{~min})$. The resin was then treated with a 80:20 mixture of DMF and piperidine $(2 \times 10 \mathrm{~min})$ to remove the Fmoc protecting group of the Aze $(\mathrm{Se}, \mathrm{S})$ amino acid. Finally the solid support was washed with DMF $(3 \times 2 \mathrm{~min})$.

\section{Final peptide deprotection and cleavage}

At the end of the synthesis, the resin was washed with $\mathrm{CH}_{2} \mathrm{Cl}_{2}(3 \times 2 \mathrm{~min})$ and diethyl ether ( 3 $\times 2$ min) and dried in vacuo. The crude peptide was cleaved from the solid support by reaction TFA cocktails, precipitated by addition of cold diethyl ether/heptane 1:1 (20 mL per mL of TFA cocktail) and recovered by centrifugation.

\section{Oxidation of the SEA group $\left(S E A^{\text {on }} \rightarrow S E A^{\text {off }}\right)$}

The SEA ${ }^{\text {on }}$ peptide recovered after precipitation was solubilized in deionised water, lyophilized and dissolved in AcOH/water 1:4. A solution of $\mathrm{I}_{2}$ in DMSO $(\approx 100 \mathrm{mg} / \mathrm{mL})$ was added dropwise until complete oxidation of the SEA group (persistence of the yellow color of iodine in the reaction mixture). After $30 \mathrm{~s}$ stirring, a solution of DTT in AcOH/water: $1 / 4 \approx 100$ $\mathrm{mg} / \mathrm{mL}$ ) was added to consume the excess of $\mathrm{I}_{2}$. Then the peptide was immediately purified by HPLC.

\section{Transthioesterification $\left(S E A^{o n} \rightarrow M P A\right)$}

The SEA ${ }^{\text {on }}$ peptide recovered after precipitation was converted into the corresponding MPA thioester by reaction with MPA (5\% in volume) at $\mathrm{pH} 4.0$. This reaction was performed in absence of TCEP when the SetCys amino acid is included in the peptide sequence (TCEP induces the conversion of the SetCys amino acid into cysteine). A detailed procedure is given below for the synthesis of MPA thioesters. 


\subsection{Synthesis of SetCys peptides}

\section{SetCys peptide used for exploring SetCys reactivity (Figure 2 \& Figure 3)}

SetCys-ALKEPVHGV-NH2 peptide 1 was synthesized on $0.1 \mathrm{mmol}$ scale as described in the general procedure and was cleaved from the solid support using TFA/TIS/ $\mathrm{H}_{2} \mathrm{O} / \mathrm{EDT}$ 92.5:2.5:2.5:2.5 (12 $\mathrm{mL})$. The titled peptide which was recovered by precipitation from $\mathrm{Et}_{2} \mathrm{O} /$ heptane was solubilized in water and lyophilized and was used in the next step without further purification (48 mg, 32\%).

Characterization of SetCys-ALKEPVHGV-NH $\mathrm{N}_{2}$ peptide $\mathbf{1}$ :

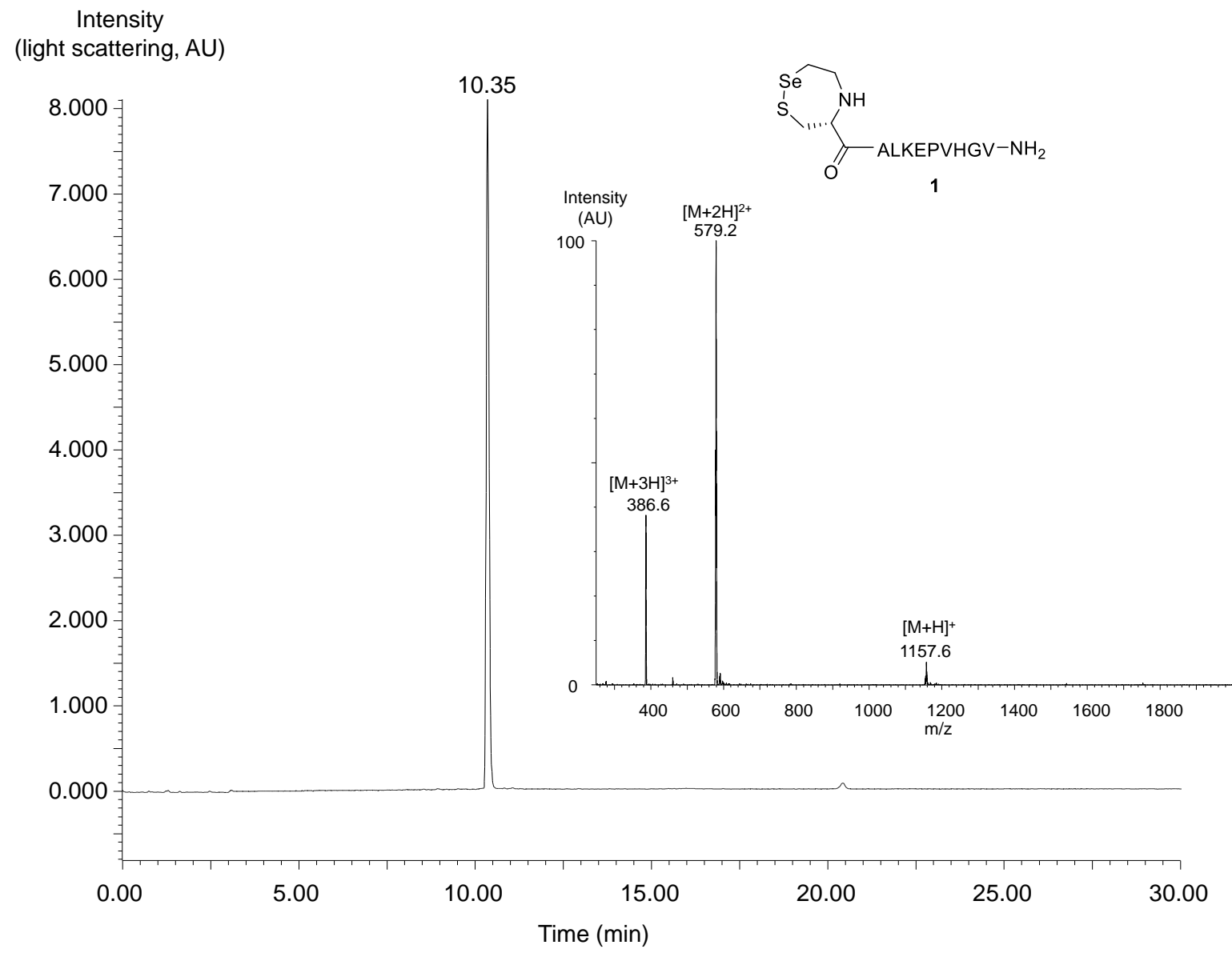

Figure S 22. LC-MS analysis of SetCys-ALKEPVHGV-NH2 peptide 1. LC trace: eluent A $0.10 \%$ TFA in water, eluent $\mathrm{B} 0.10 \%$ TFA in $\mathrm{CH}_{3} \mathrm{CN} /$ water: $4 / 1$ by vol. $\mathrm{C} 18$ column, gradient $0-100 \% \mathrm{~B}$ in $30 \mathrm{~min}, 30{ }^{\circ} \mathrm{C}, 1 \mathrm{~mL} / \mathrm{min}$, light scattering detection. MS trace: $\mathrm{m} / \mathrm{z}$ calcd. for $[\mathrm{M}+\mathrm{H}]^{+}$(monoisotopic mass): 1157.50, found: 1157.6. 


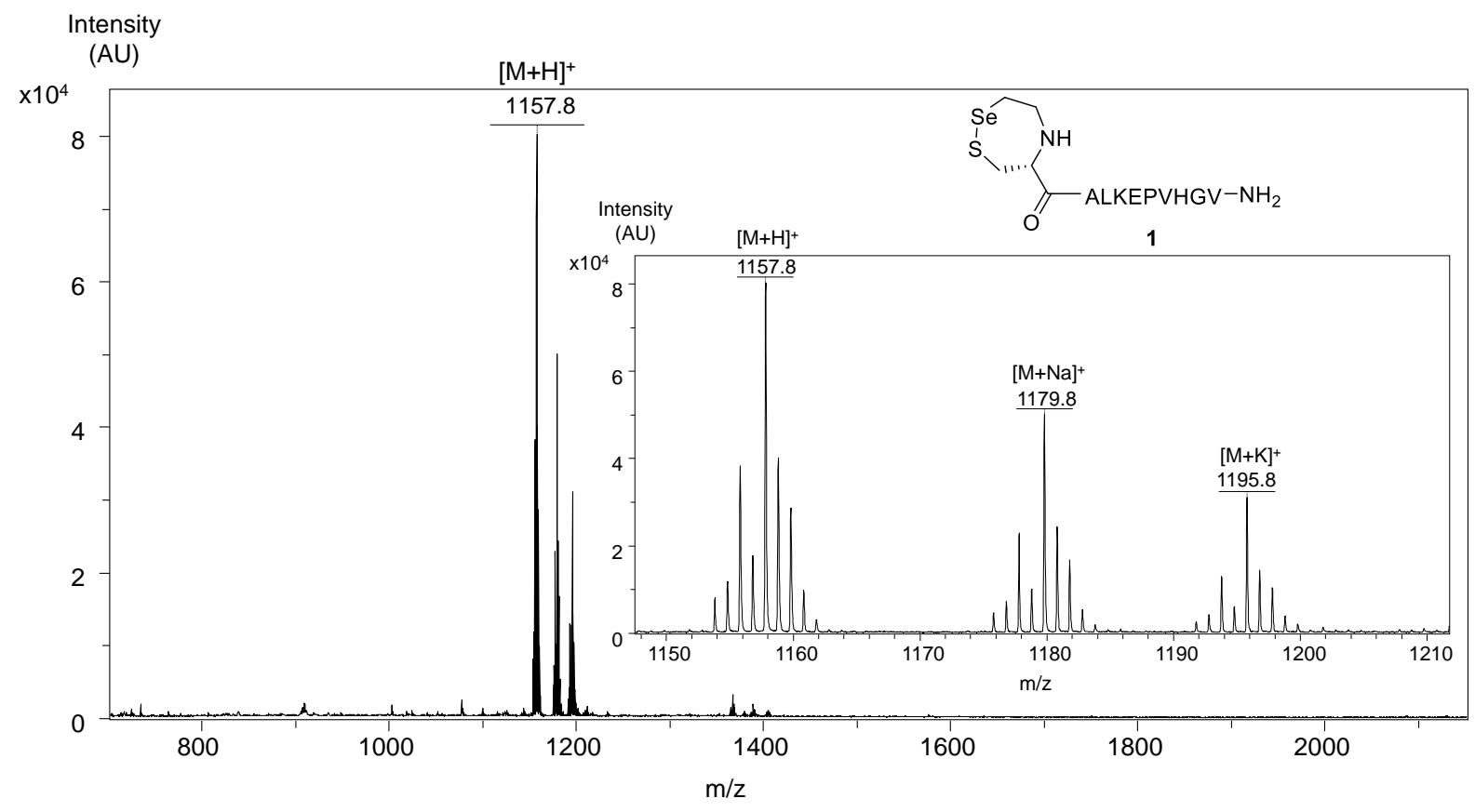

Figure S 23. MALDI-TOF analysis of SetCys-ALKEPVHGV-NH2 peptide 1. Matrix $\alpha$ cyano-4-hydroxycinnamic acid, positive detection mode, calcd. for $[\mathrm{M}+\mathrm{H}]^{+}$(monoisotopic): 1157.50, found: 1157.8 .

\section{SetCys peptides for cyclization studies (Figure 5)}

\section{Synthesis of peptide 8a}

SetCys-ALKEPVHGA-MPA peptide 8a was synthesized on $0.08 \mathrm{mmol}$ scale as described in the general procedure. TFA/ $\mathrm{H}_{2} \mathrm{O} / \mathrm{TIS} /$ thiophenol 92.5:2.5:2.5:2.5 $(8 \mathrm{~mL})$ was used as cleavage cocktail. The $\mathrm{SEA}^{\text {on }}$ peptide which was recovered by precipitation from $\mathrm{Et}_{2} \mathrm{O} /$ heptane was immediately converted into the corresponding MPA thioester using the following procedure.

MPA ( $1 \mathrm{~mL})$ was dissolved in $19 \mathrm{~mL}$ of water and the $\mathrm{pH}$ of the solution was adjusted to 4.0 by addition of $6 \mathrm{M} \mathrm{NaOH}$. The $\mathrm{SEA}^{\text {on }}$ peptide recovered after acidic cleavage was directly dissolved in this solution of MPA and the reaction mixture was stirred under inert atmosphere (glovebox) at $37^{\circ} \mathrm{C}$ for $16 \mathrm{~h}$. The reaction mixture was then acidified with $10 \% \mathrm{AcOH}$ in water $(10 \mathrm{~mL})$ and extracted with $\mathrm{Et}_{2} \mathrm{O}(5 \times)$ to remove the MPA. Purification of the crude by semipreparative HPLC (eluent A $0.10 \%$ TFA in water, eluent B $0.10 \%$ TFA in $\mathrm{CH}_{3} \mathrm{CN} /$ water: $4 / 1$ by vol, $6 \mathrm{~mL} / \mathrm{min}, 0-35 \%$ eluent $\mathrm{B}$ in $25 \mathrm{~min}$, C18 XBridge $5 \mu \mathrm{m}(10 \times 250 \mathrm{~mm})$ column, detection at $215 \mathrm{~nm}, 30^{\circ} \mathrm{C}$ ) provided SetCys-ALKEPVHGA-MPA peptide 8a as a white solid after lyophilisation $(25.3 \mathrm{mg}, 20 \%)$. 


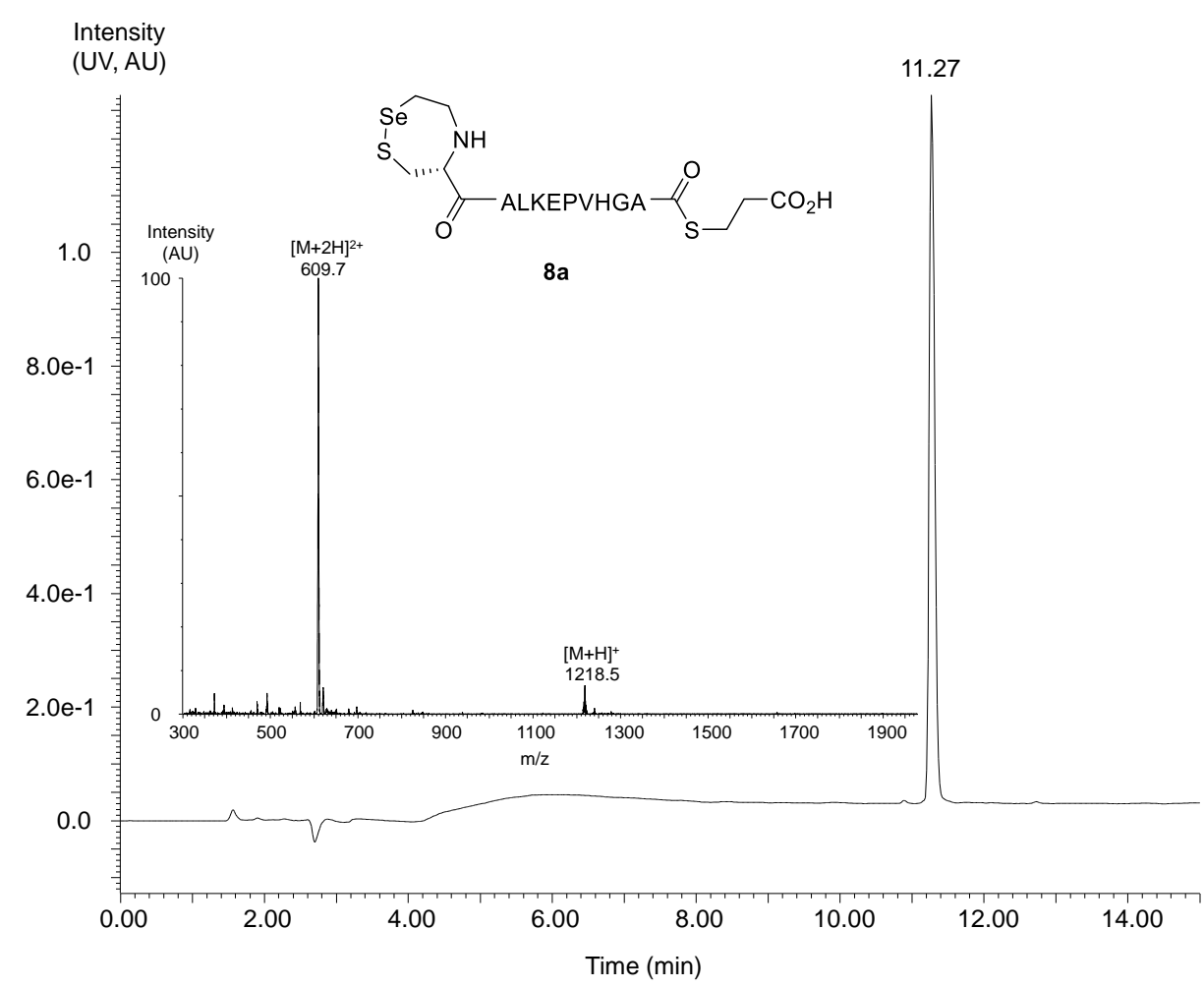

Figure S 24. LC-MS analysis of SetCys-ALKEPVHGA-MPA peptide 8a. LC trace: eluent A $0.10 \%$ TFA in water, eluent $\mathrm{B} 0.10 \%$ TFA in $\mathrm{CH}_{3} \mathrm{CN} /$ water: $4 / 1$ by vol. $\mathrm{C} 18$ column, gradient $0-50 \% \mathrm{~B}$ in $15 \mathrm{~min}, 30{ }^{\circ} \mathrm{C}, 1 \mathrm{~mL} / \mathrm{min}$, UV detection. MS trace: $\mathrm{m} / \mathrm{z}$ calcd. for $[\mathrm{M}+\mathrm{H}]^{+}$ (monoisotopic mass): 1218.45, found: 1218.5 .

\section{Synthesis of peptide $8 \mathrm{~b}$}

SetCys-(GS)2-K(Biot)-(GS)2A-MPA peptide 8b was synthesized on $0.05 \mathrm{mmol}$ scale as described in the general procedure. TFA/ $\mathrm{H}_{2} \mathrm{O} / \mathrm{TIS} /$ thiophenol 92.5:2.5:2.5:2.5 (5 mL) was used as cleavage cocktail. The $\mathrm{SEA}^{\text {on }}$ peptide which was recovered by precipitation from $\mathrm{Et}_{2} \mathrm{O} /$ heptane was immediately converted into the corresponding MPA thioester using the following procedure.

MPA $(0.62 \mathrm{~mL})$ was dissolved in water $(11.88 \mathrm{~mL})$ and the $\mathrm{pH}$ of the solution was adjusted to 4.0 by addition of $6 \mathrm{M} \mathrm{NaOH}$. The $\mathrm{SEA}^{\text {on }}$ peptide recovered after acidic cleavage was dissolved in this solution of MPA and the reaction mixture was stirred under inert atmosphere (glovebox) at $37^{\circ} \mathrm{C}$ for $16 \mathrm{~h}$. The reaction mixture was then acidified with $1.5 \%$ TFA in water $(9 \mathrm{~mL})$ and was extracted with $\mathrm{Et}_{2} \mathrm{O}(5 \times)$ to remove the MPA. Purification of the crude by semi-preparative HPLC (eluent A $0.10 \%$ TFA in water, eluent B $0.10 \%$ TFA in $\mathrm{CH}_{3} \mathrm{CN} /$ water: 4/1 by vol, 6 $\mathrm{mL} / \mathrm{min}, 0-5 \%$ eluent $\mathrm{B}$ in 3 min then 5-30\% eluent B in $32 \mathrm{~min}, \mathrm{C} 18$ XBridge $5 \mu \mathrm{m}(10 \times 250$ $\mathrm{mm}$ ) column, detection at $215 \mathrm{~nm}, 30{ }^{\circ} \mathrm{C}$ ) provided SetCys-(GS)2-K(Biot)-(GS)2A-MPA peptide $\mathbf{8 b}$ as a white solid after lyophilisation (5.48 $\mathrm{mg}, 8 \%$ ). 
Characterization of SetCys- $(G S)_{2}-K($ Biot $)-(G S)_{2} A-M P A$ peptide $8 \boldsymbol{b}$ :

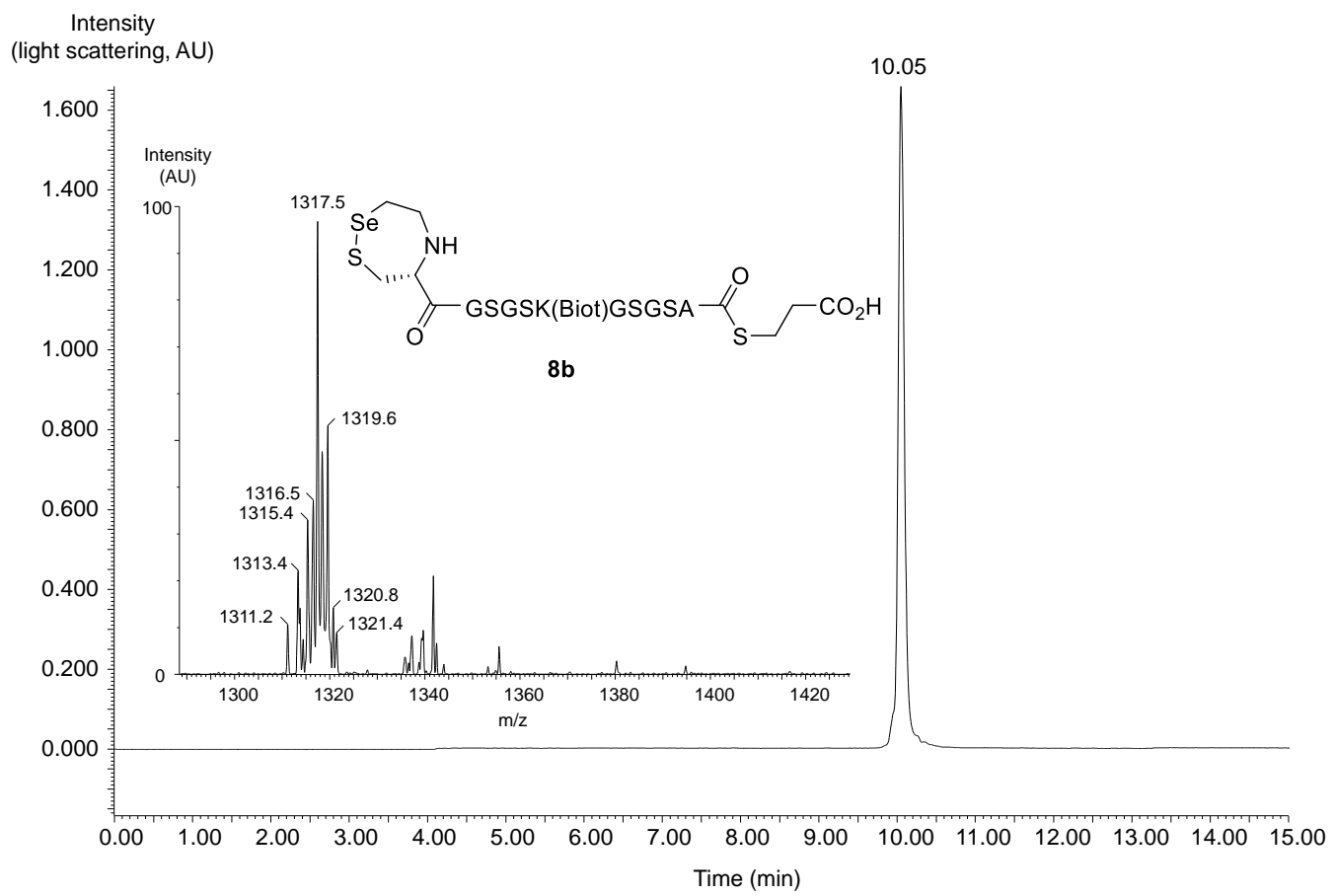

Figure S 25. LC-MS analysis of SetCys-(GS)2-K(Biot)-(GS) 2 A-MPA peptide $8 \mathrm{~b}$. LC trace: eluent A $0.10 \%$ TFA in water, eluent $\mathrm{B} 0.10 \%$ TFA in $\mathrm{CH}_{3} \mathrm{CN} /$ water: $4 / 1$ by vol. $\mathrm{C} 18$ column, gradient $0-50 \% \mathrm{~B}$ in $15 \mathrm{~min}, 30{ }^{\circ} \mathrm{C}, 1 \mathrm{~mL} / \mathrm{min}$, light scattering detection. MS trace: $\mathrm{m} / \mathrm{z}$ calcd. for $[\mathrm{M}+\mathrm{H}]^{+}$(monoisotopic mass): 1317.37, found: 1317.5.

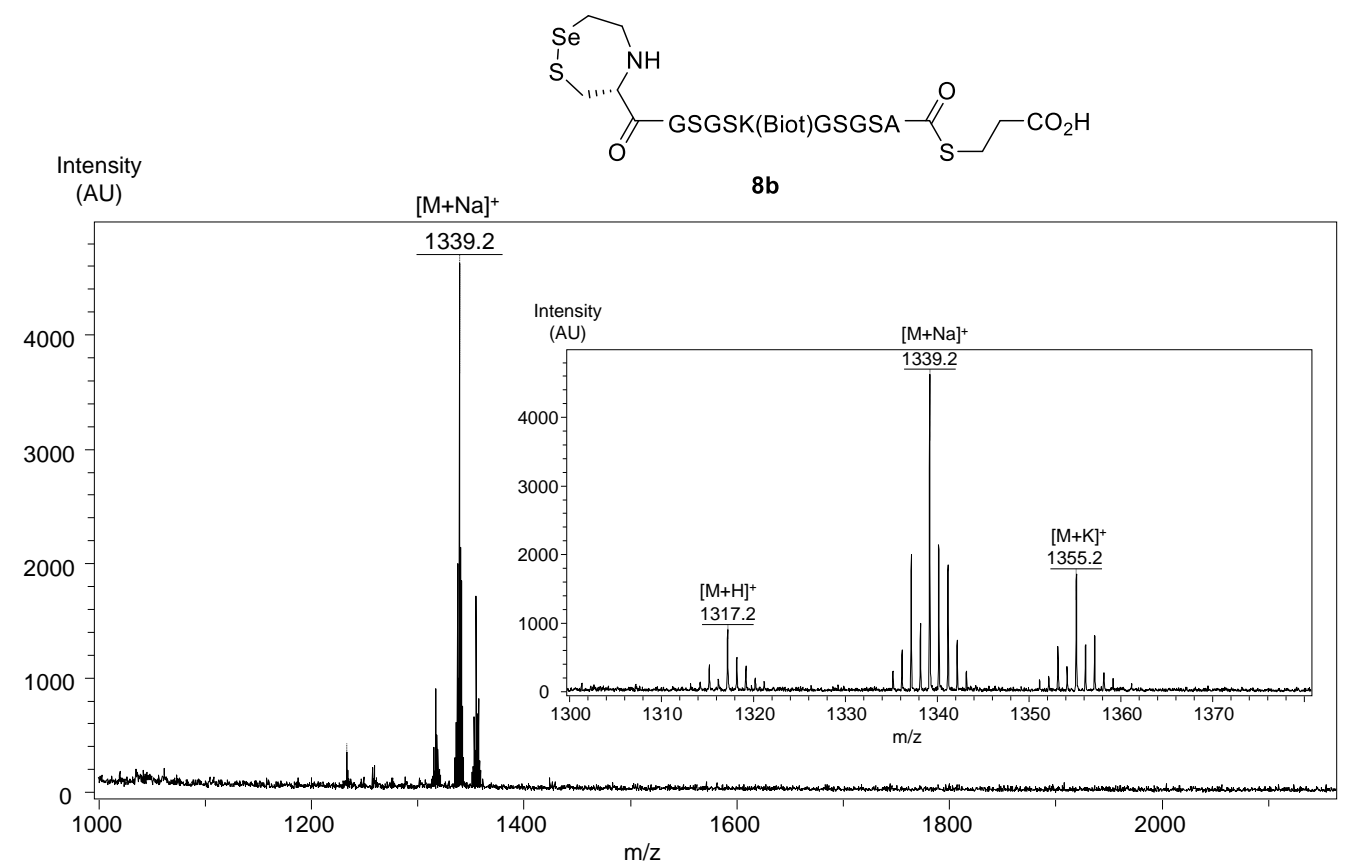

Figure S 26. MALDI-TOF analysis of SetCys-(GS)2-K(Biot)-(GS)2A-MPA peptide $8 \mathrm{~b}$. Matrix $\alpha$-cyano-4-hydroxycinnamic acid, positive detection mode, calcd. for $[\mathrm{M}+\mathrm{Na}]^{+}$ (monoisotopic): 1339.37, found: 1339.2. 


\section{Synthesis of peptide $8 \mathrm{c}$}

SetCys-(GS)3-K(Biot)-(GS)3A-MPA peptide 8c was synthesized on $0.05 \mathrm{mmol}$ scale as described in the general procedure. TFA/ $\mathrm{H}_{2} \mathrm{O} / \mathrm{TIS} /$ thiophenol 92.5:2.5:2.5:2.5 (5 mL) was used as cleavage cocktail. The $\mathrm{SEA}^{\text {on }}$ peptide which was recovered by precipitation from $\mathrm{Et}_{2} \mathrm{O} /$ heptane was immediately converted into the corresponding MPA thioester using the following procedure.

MPA (1.25 mL) was dissolved in water $(23.75 \mathrm{~mL})$ and the $\mathrm{pH}$ of the solution was adjusted to 4.0 by addition of $6 \mathrm{M} \mathrm{NaOH}$. The $\mathrm{SEA}^{\text {on }}$ peptide recovered after acidic cleavage was dissolved in this solution of MPA and the reaction mixture was stirred at $37{ }^{\circ} \mathrm{C}$ for $5.5 \mathrm{~h}$. The reaction was monitored by HPLC and was stopped before completion to limit the formation of the side product which results from the slow cyclisation of SetCys-(GS)3-K(Biot)-(GS)3A-MPA peptide 8c. The reaction mixture was then acidified with 10\% TFA in water (until pH 3.2) and was extracted with $\mathrm{Et}_{2} \mathrm{O}(5 \times)$ to remove the MPA. Purification of the crude by semi-preparative HPLC (eluent A $0.10 \%$ TFA in water, eluent B $0.10 \%$ TFA in $\mathrm{CH}_{3} \mathrm{CN} /$ water: 4/1 by vol, 6 $\mathrm{mL} / \mathrm{min}, 0-5 \%$ eluent $\mathrm{B}$ in 3 min then 5-35\% eluent B in $32 \mathrm{~min}, \mathrm{C} 18$ XBridge $5 \mu \mathrm{m}(10 \times 250$ $\mathrm{mm}$ ) column, detection at $215 \mathrm{~nm}, 30{ }^{\circ} \mathrm{C}$ ) provided SetCys-(GS)3-K(Biot)-(GS)3A-MPA peptide $8 \mathbf{c}$ as a white solid after lyophilisation (16.6 $\mathrm{mg}, 19 \%)$.

Characterization of SetCys- $(G S)_{3}-K($ Biot $)-(G S)_{3} A-M P A$ peptide 8c:

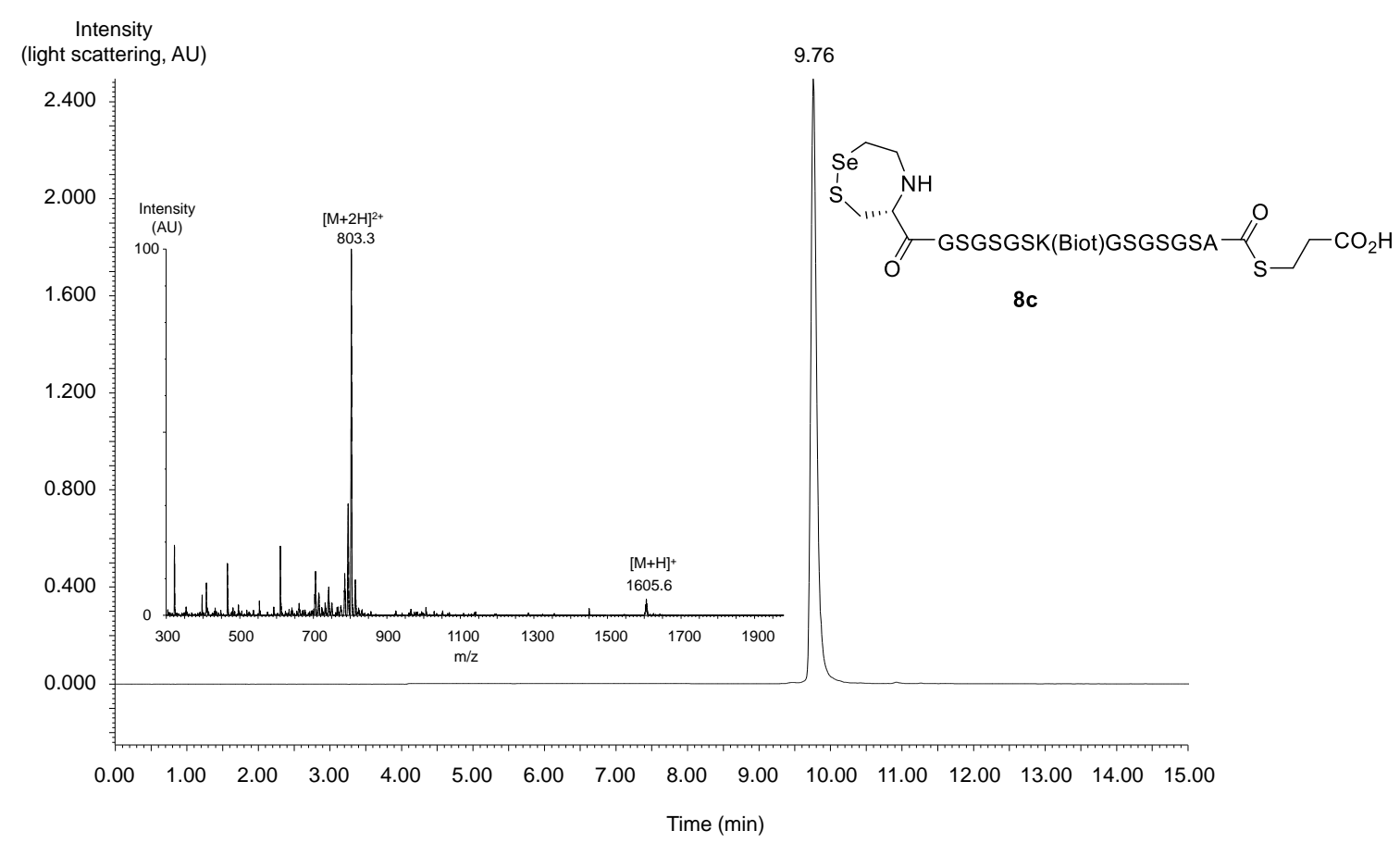

Figure S 27. LC-MS analysis of SetCys-(GS)3-K(Biot)-(GS)3A-MPA peptide 8c. LC trace: eluent $\mathrm{A} 0.10 \%$ TFA in water, eluent $\mathrm{B} 0.10 \%$ TFA in $\mathrm{CH}_{3} \mathrm{CN} /$ water: $4 / 1$ by vol. $\mathrm{C} 18$ column, gradient $0-50 \% \mathrm{~B}$ in $15 \mathrm{~min}, 30{ }^{\circ} \mathrm{C}, 1 \mathrm{~mL} / \mathrm{min}$, light scattering detection. MS trace: $\mathrm{m} / \mathrm{z}$ calcd. for $[\mathrm{M}+\mathrm{H}]^{+}$(monoisotopic mass): 1605.48, found: 1605.6. 


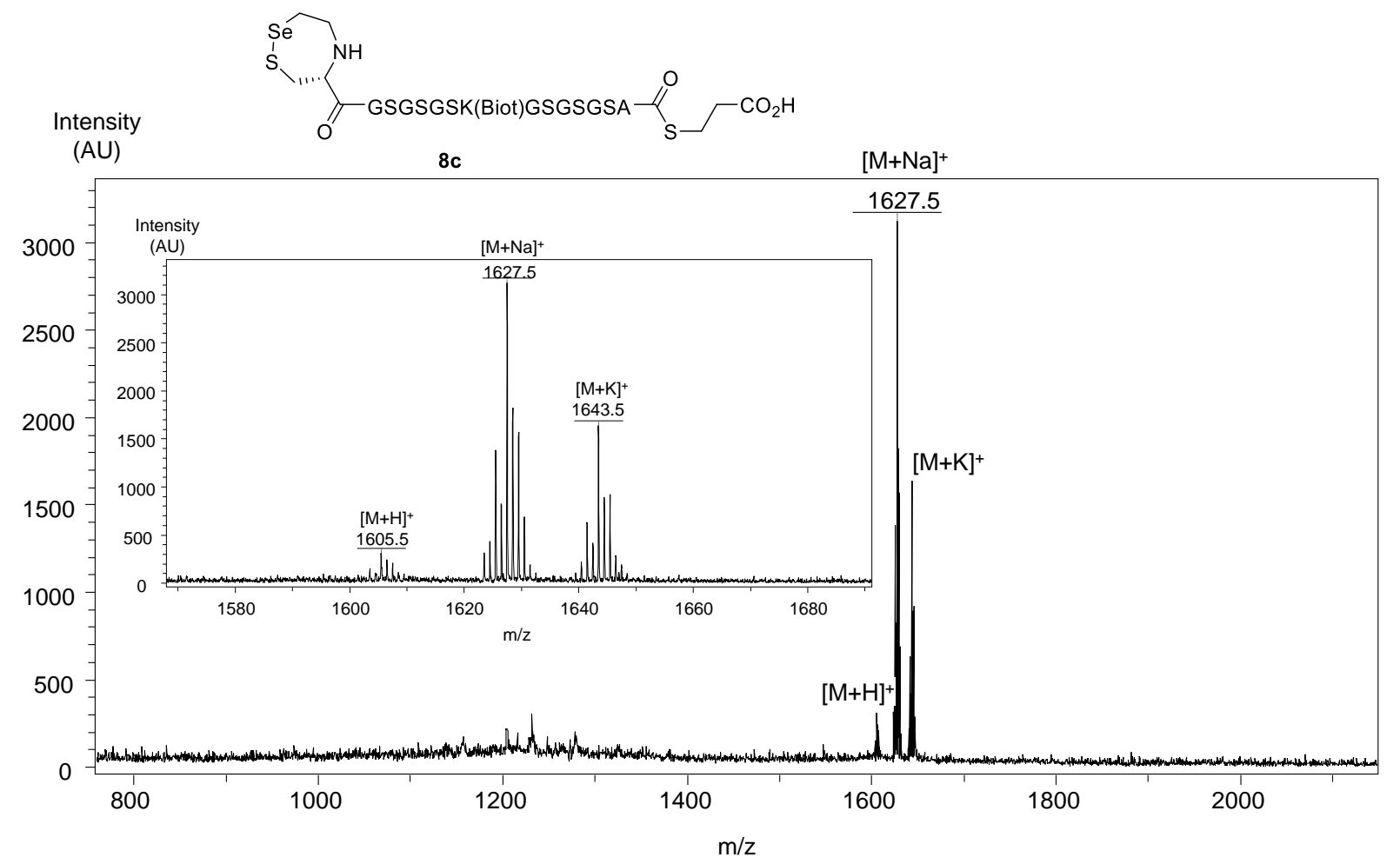

Figure S 28. MALDI-TOF analysis of SetCys-(GS)3-K(Biot)-(GS)3A-MPA peptide 8c. Matrix $\alpha$-cyano-4-hydroxycinnamic acid, positive detection mode, calcd. for $[\mathrm{M}+\mathrm{Na}]^{+}$ (monoisotopic): 1627.46, found: 1627.5.

\subsection{Preparation of SetAla peptide (Figure 3d)}

Synthesis of Fmoc-SetAla-OH SII2

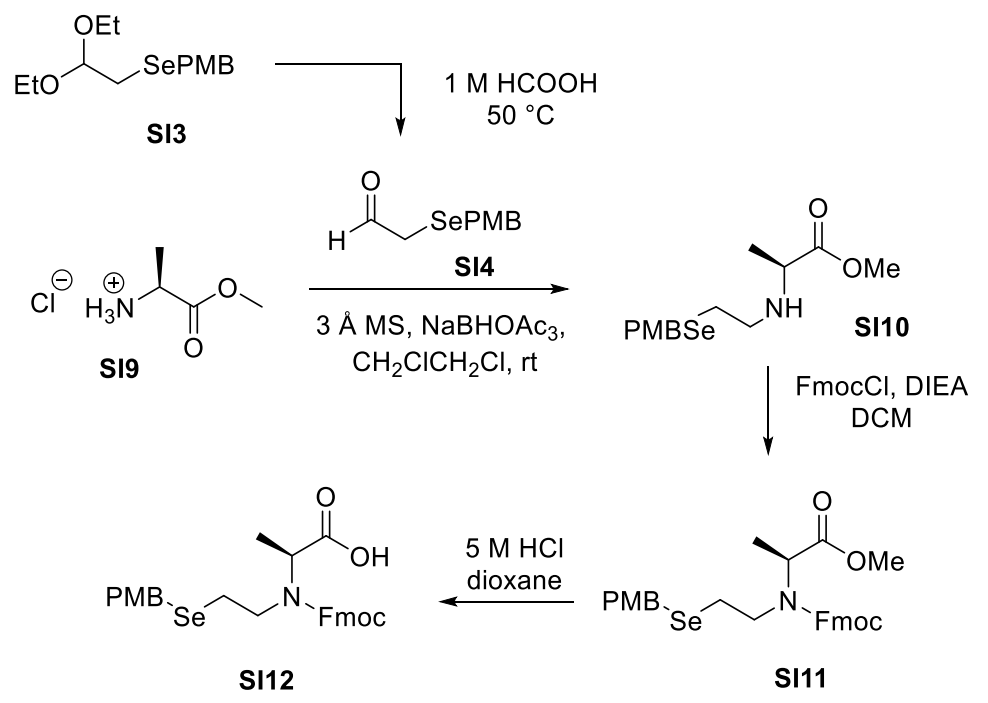

Figure S 29. Synthesis of the Fmoc-protected SetAla amino acid SI12. 
(2,2-Diethoxyethyl)(4-methoxybenzyl)selenide SI3 (230 $\mathrm{mg}, 0.73 \mathrm{mmol}$ ) was suspended in 1 $\mathrm{M}$ formic acid $(6 \mathrm{~mL})$ and the mixture was heated overnight at $50{ }^{\circ} \mathrm{C}$. Water $(50 \mathrm{~mL})$ was added and the aqueous layer was extracted with $\mathrm{Et}_{2} \mathrm{O}(2 \times 50 \mathrm{~mL})$. The combined organic extracts were washed with water $(50 \mathrm{~mL})$ and dried over $\mathrm{MgSO}_{4}$. After evaporation of the solvent under reduced pressure, aldehyde SI4 was dissolved in anhydrous 1,2-dichloroethane (15 mL) under argon. H-Ala-OMe.HCl SI9 (106 mg, $0.76 \mathrm{mmol}$ ), DIEA (146 $\mu \mathrm{L}, 0.84 \mathrm{mmol}$, activated powdered $3 \AA$ molecular sieves $(760 \mathrm{mg}$ ) and sodium triacetoxyborohydride (208 $\mathrm{mg}$, $0.98 \mathrm{mmol}$ ) were successively added to the solution of aldehyde. After $20 \mathrm{~h}$ stirring at RT, the reaction mixture was filtered on a Büchner funnel and the solid was washed with additional portions of DCM. The filtrate was evaporated under reduced pressure. Then $0.5 \mathrm{M} \mathrm{K}_{2} \mathrm{CO}_{3}(30$ $\mathrm{mL})$ and saturated $\mathrm{NaCl}(30 \mathrm{~mL})$ were added to the residue and the aqueous layer was extracted with DCM $(3 \times 50 \mathrm{~mL})$. The combined organic layers were dried over $\mathrm{MgSO}_{4}$ and the solvent was evaporated under reduced pressure.

The $N$-alkylated derivative SI10 contained in the residue $(272 \mathrm{mg}$ ) was dissolved in DCM (4 $\mathrm{mL})$. DIEA (0.14 mL, $0.82 \mathrm{mmol})$ and $\mathrm{FmocCl}(212 \mathrm{mg}, 0.82 \mathrm{mmol})$ were added to the solution of SI10. After $24 \mathrm{~h}$ stirring at RT, the reaction mixture was diluted with an additional portion of DCM. The organic layer was washed with water and was dried over $\mathrm{MgSO}_{4}$. After evaporation of the solvent under reduced pressure, purification of the crude by column chromatography (cyclohexane/EtOAc 7:3) provided the Fmoc-protected derivative SI11, in mixture with an impurity that resulted from $\mathrm{FmocCl}$ decomposition $(310 \mathrm{mg})$.

To the compound SI11 isolated by column chromatography (140 $\mathrm{mg}$ ) were added dioxane (6 $\mathrm{mL})$ and $5 \mathrm{M} \mathrm{HCl}(3 \mathrm{~mL})$ and the mixture was refluxed for $6 \mathrm{~h}$. The reaction mixture was then diluted with $1 \mathrm{M} \mathrm{HCl}(40 \mathrm{~mL})$ and the aqueous layer was extracted with DCM $(3 \times 40 \mathrm{~mL})$. The combined organic layers were dried over $\mathrm{MgSO}_{4}$ and the solvent was evaporated under reduced pressure. Purification of the crude by column chromatography (DCM/MeOH 99:1 $\rightarrow$ 95:5) provided the expected amino acid SI12 as a glass (55 $\mathrm{mg}$, 31\% from SI3).

The titled compound was characterized by ${ }^{1} \mathrm{H}$ and ${ }^{13} \mathrm{C}$ NMR as a mixture of conformers. Due to overlapping signals, no clear description of the ${ }^{1} \mathrm{H}$ NMR spectrum can be provided.

IR $\left(A T R, \mathrm{~cm}^{-1}\right) 3065,2995,2932,1739,1696,1509,1284,1244,1172,1101,726 .[\boldsymbol{\alpha}]_{\mathbf{D}^{\mathbf{2 0}}}(\mathrm{c}$ $\left.1.0, \mathrm{CHCl}_{3}\right):-9^{\circ}$. 

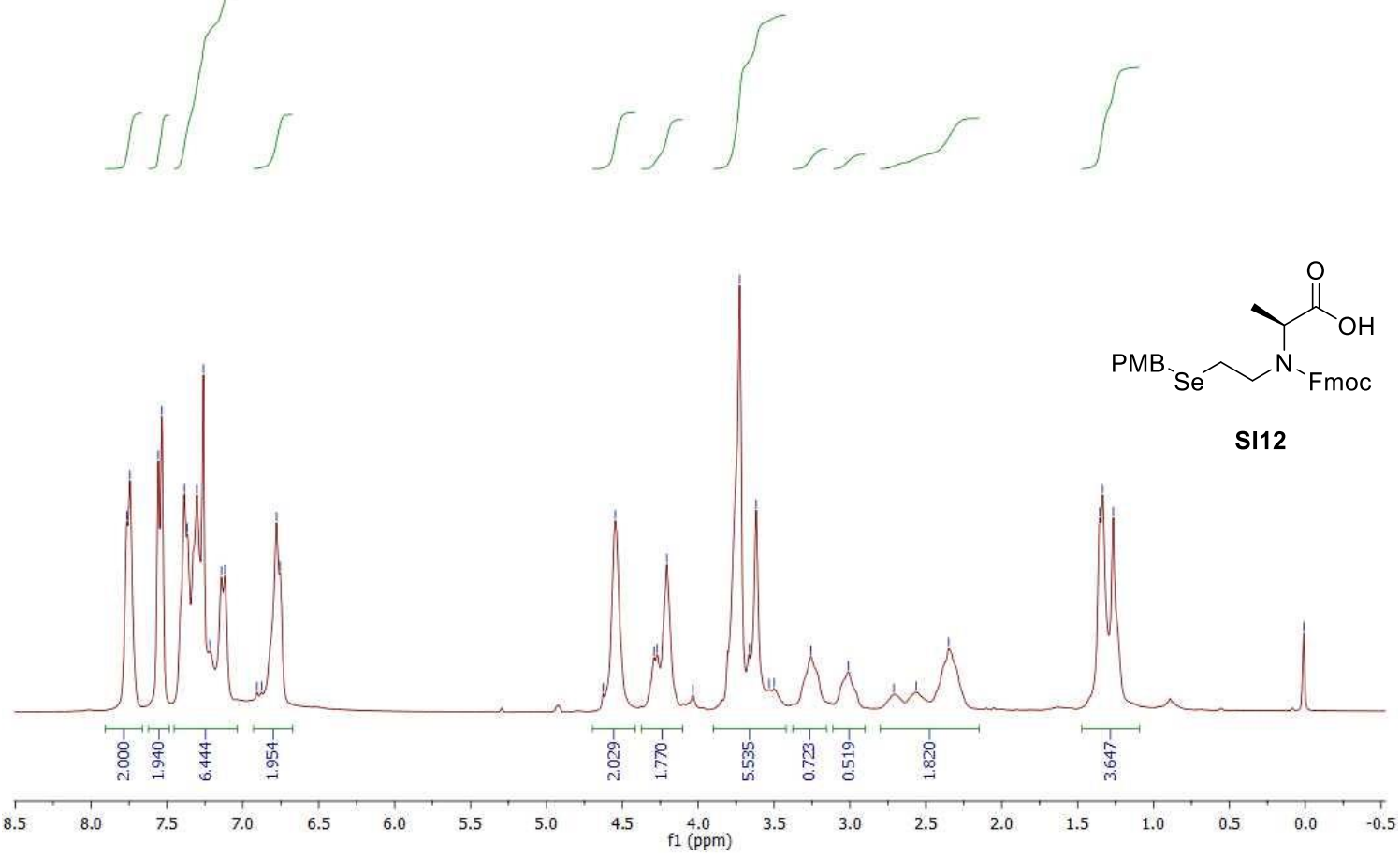

Figure S 30. ${ }^{1} \mathrm{H}$ NMR (300 MHz) spectrum of Fmoc-SetAla-OH SI12 (CDCl 3 , $\left.293 \mathrm{~K}\right)$.

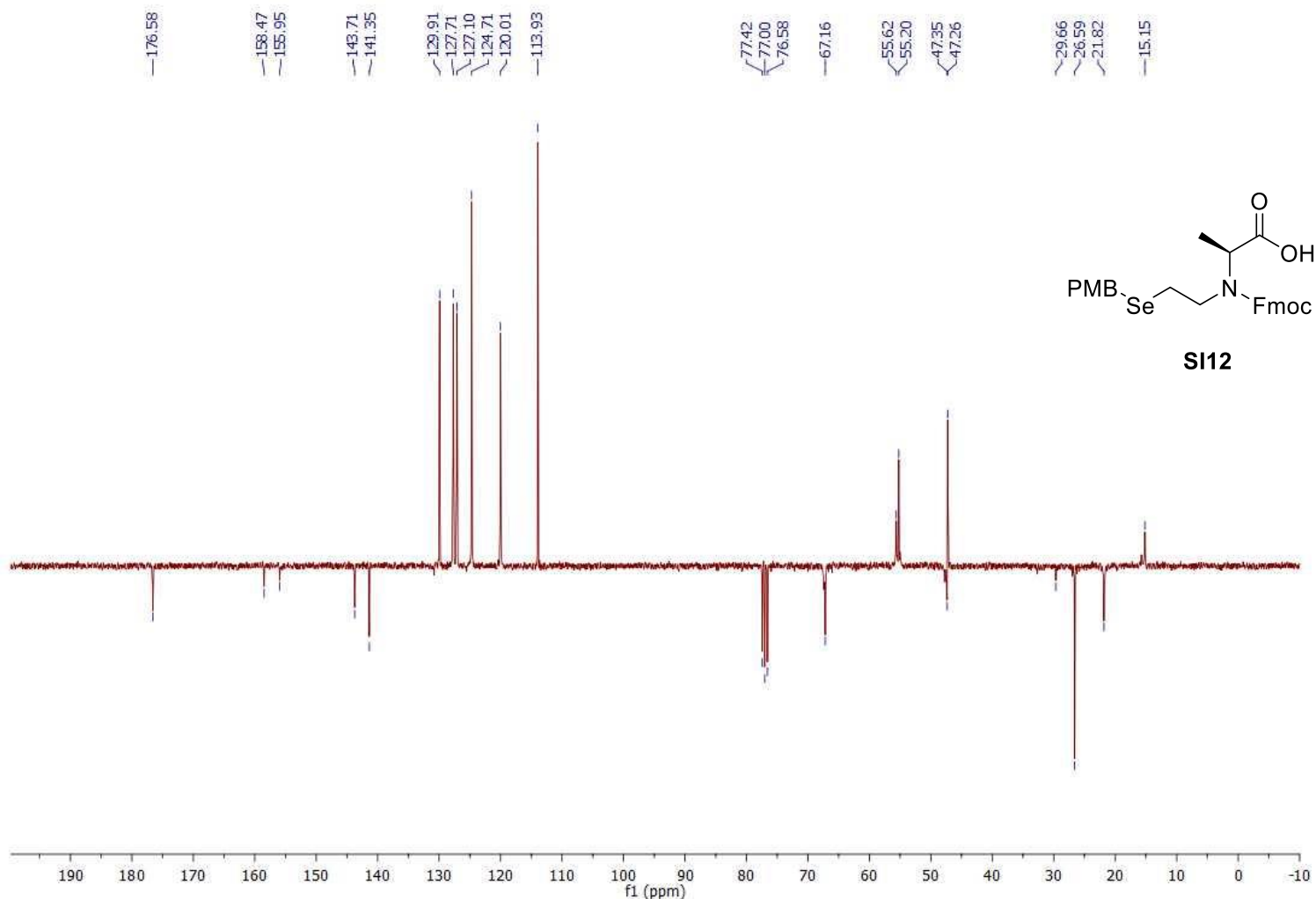

Figure S 31. ${ }^{13} \mathrm{C}$ NMR (75 MHz) spectrum of Fmoc-SetAla-OH SI12 (CDCl, $\left.293 \mathrm{~K}\right)$. 


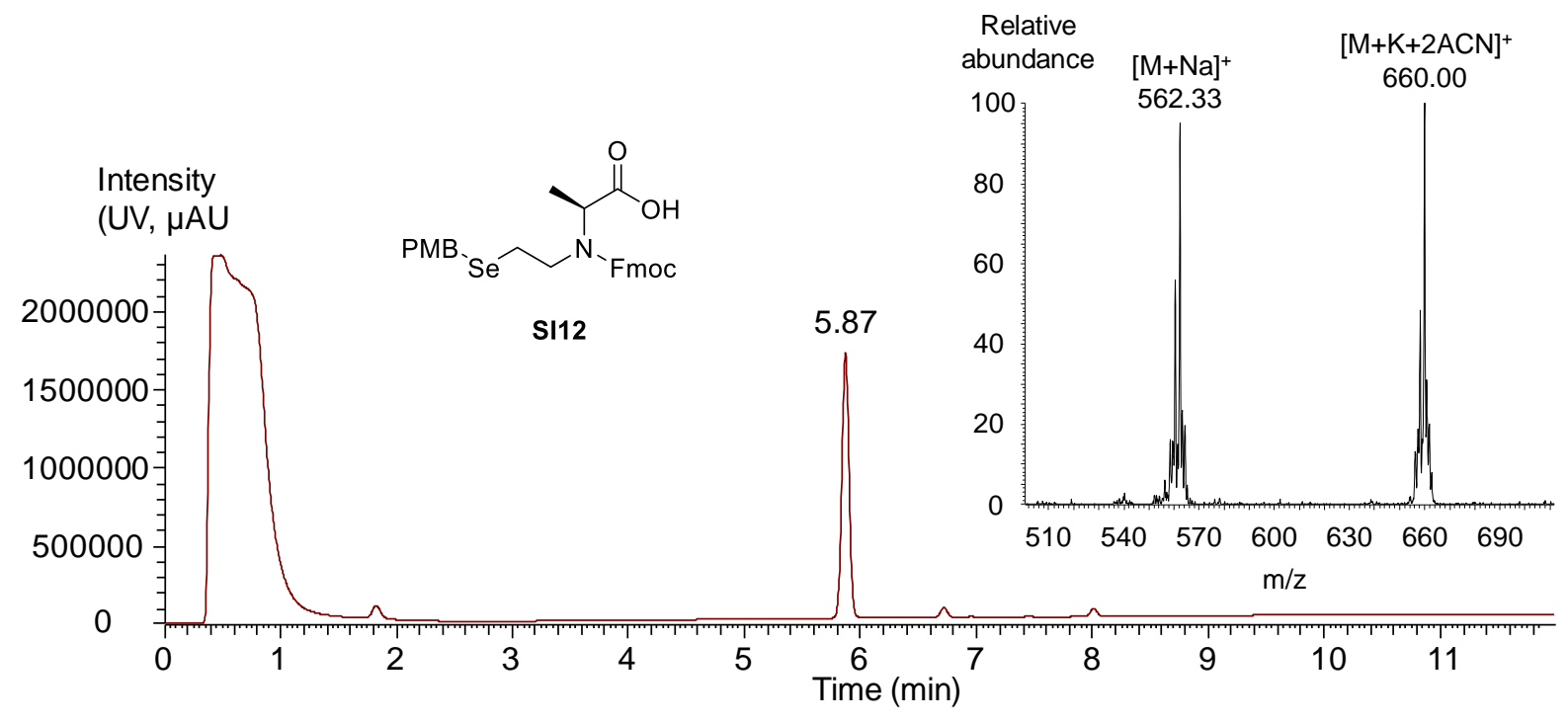

Figure S 32. LC-MS analysis of Fmoc-SetAla-OH SI12. LC trace: eluent C $0.10 \%$ formic acid in water, eluent $\mathrm{D} 0.10 \%$ formic acid in $\mathrm{CH}_{3} \mathrm{CN}$, gradient $40-100 \% \mathrm{D}$ in $10 \mathrm{~min}, 0.4 \mathrm{~mL} / \mathrm{min}$, Atlantis T3 $3 \mu \mathrm{m} 2.1 \times 50 \mathrm{~mm}$ column; $50{ }^{\circ} \mathrm{C}$; UV detection. MS trace: $[\mathrm{M}+\mathrm{Na}]^{+} \mathrm{m} / \mathrm{z}$ calcd. (monoisotopic mass) 562.11, found 562.33.

Elongation of the SetAla peptide

SetAla peptide was elongated according to general procedure presented in section 3.1. Note that a protocol similar to the one described for SetCys was used to couple the SetAla amino acid at the N-terminus of the elongated peptide.

Cleavage of the SetAla peptide

PMB-SeCH2 $\mathbf{C H}_{2}$-Ala-ALKEPVHGV-NH2 peptide was synthesized on $0.05 \mathrm{mmol}$ scale. TFA/ $\mathrm{H}_{2} \mathrm{O} / \mathrm{TIS}$ 95:2.5:2.5 (5 mL) was used as cleavage cocktail.

Removal of PMB protecting group

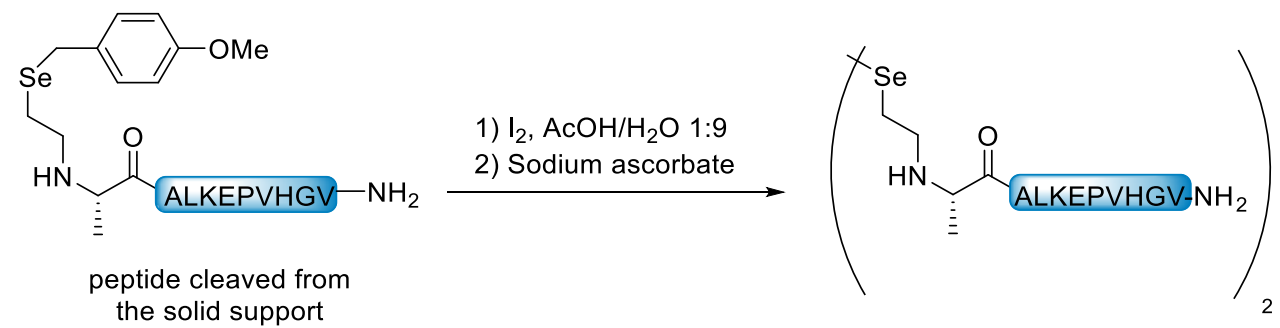

Figure S 33. Removal of PMB protecting group.

The PMB-protected peptide which was recovered by precipitation from $\mathrm{Et}_{2} \mathrm{O} / \mathrm{heptane}$ (43.0 mg) was converted into the final diselenide by oxidation with iodine using the following procedure. The crude recovered after precipitation $(21.0 \mathrm{mg}$ ) was solubilized in $\mathrm{AcOH} /$ water 1:4 $(13.2 \mathrm{~mL})$ and a solution of $\mathrm{I}_{2}$ in DMSO $(109 \mu \mathrm{L}, 61.4 \mathrm{mg} / \mathrm{mL})$ was added dropwise. After $30 \mathrm{~min}$ stirring 
at RT, a solution of sodium ascorbate in water $(9.9 \mathrm{mg} / \mathrm{mL})$ was added dropwise until the complete disappearance of $\mathrm{I}_{2}$ color. Purification of the crude by preparative HPLC (eluent A $0.10 \%$ TFA in water, eluent $\mathrm{B} 0.10 \%$ TFA in $\mathrm{CH}_{3} \mathrm{CN}, 20 \mathrm{~mL} / \mathrm{min}, 0-10 \%$ eluent $\mathrm{B}$ in 4 min then $10-30 \%$ eluent B in 29 min, C18 XBridge $5 \mu \mathrm{m}(19 \times 150 \mathrm{~mm})$ column, detection at 215 $\mathrm{nm}, \mathrm{RT})$ provided the expected diselenide as a white solid after lyophilisation $(8.7 \mathrm{mg}, 24 \%$ overall starting from solid support).

Characterization of the diselenide (SetAla-ALKEPVHGV-NH$)_{2}$ :

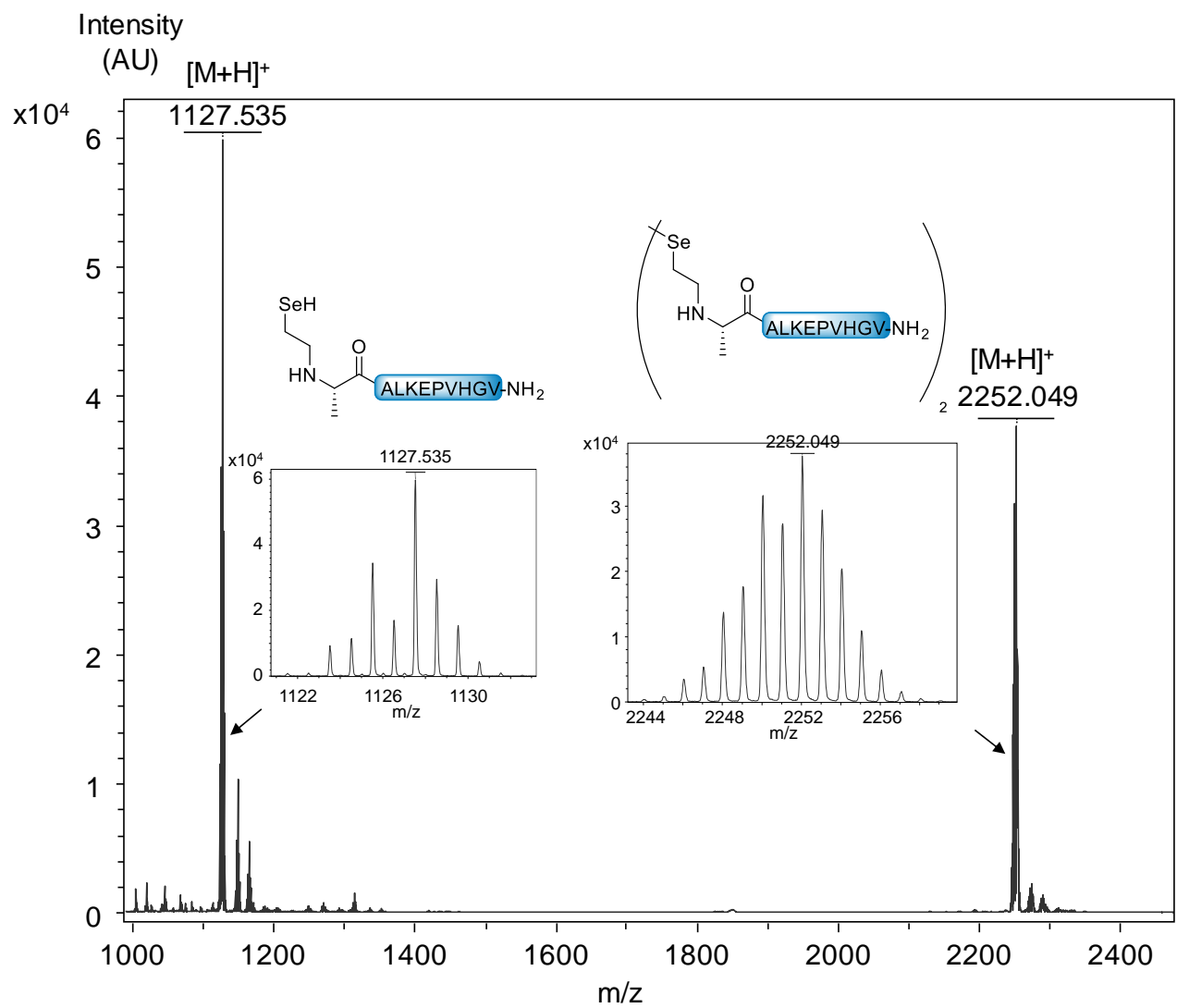

Figure S 34. MALDI-TOF analysis of the diselenide (SetAla-ALKEPVHGV-NH2)2. Matrix $\alpha$-cyano-4-hydroxycinnamic acid, positive detection mode. The diselenide as well as the selenol are detected by MALDI-TOF analysis. Calcd. for $[\mathrm{M}+\mathrm{H}]^{+}$(selenol): 1127.55, found: 1127.53; calcd for $[\mathrm{M}+\mathrm{H}]^{+}$(diselenide): 2252.07, found: 2252.05. 


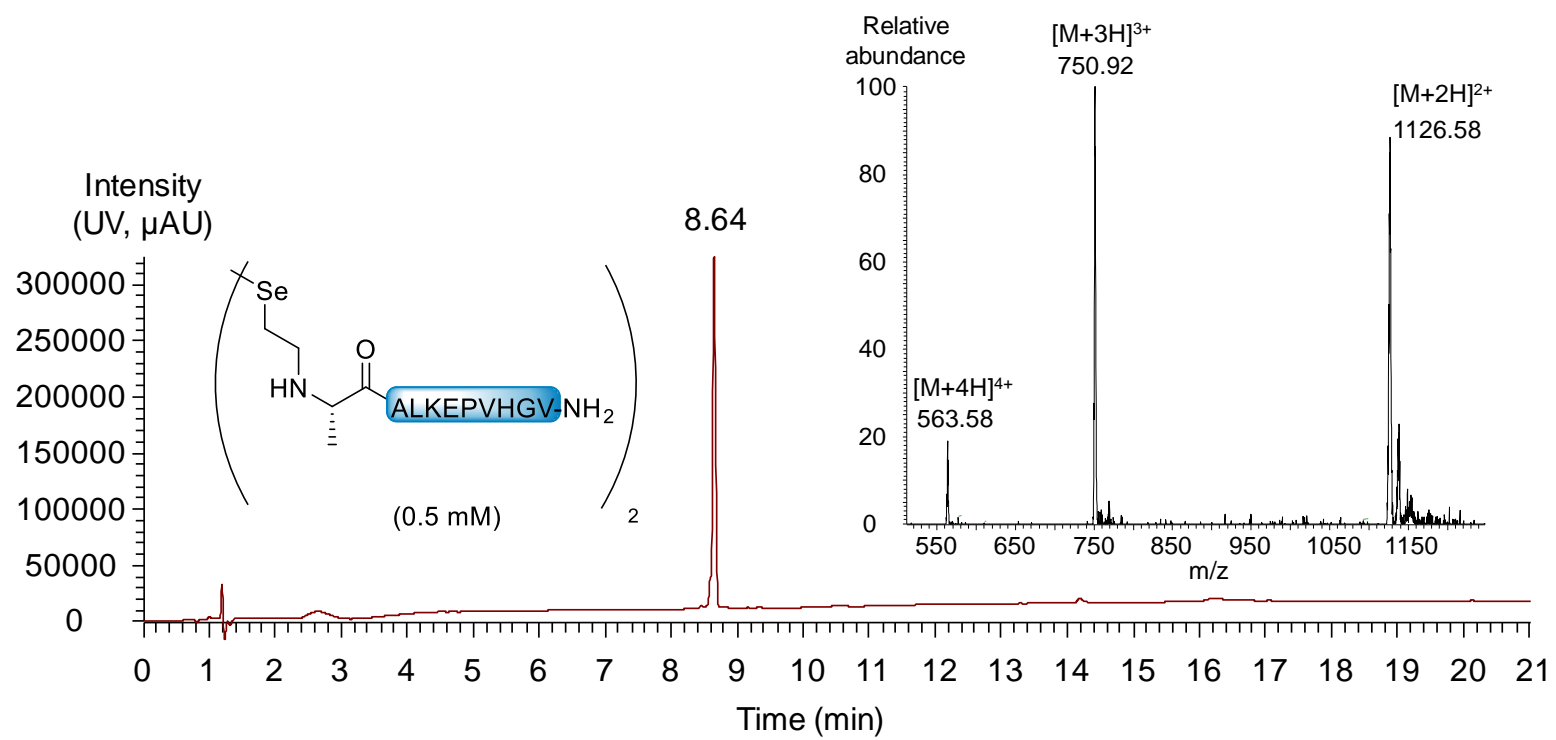

Figure S 35. LC-MS analysis of the diselenide (SetAla-ALKEPVHGV-NH2)2. LC trace: eluent A: $0.10 \%$ TFA in water, eluent B: $0.10 \%$ TFA in $\mathrm{CH}_{3} \mathrm{CN}$, gradient: $0-70 \%$ B in 20 min, $0.4 \mathrm{~mL} / \mathrm{min}$; column: AQUITY UPLC $\AA$ peptide BHE C18, $300 \AA$, $1.7 \mu \mathrm{m}, 2.1 \times 150 \mathrm{~mm} ; 50$ ${ }^{\circ} \mathrm{C}$, UV detection. MS trace: MS trace: $\mathrm{m} / \mathrm{z}=1126.6\left([\mathrm{M}+2 \mathrm{H}]^{2+}\right), 750.9\left([\mathrm{M}+3 \mathrm{H}]^{3+}\right), 563.58$ $\left([\mathrm{M}+4 \mathrm{H}]^{4+}\right)$; calcd. for $\mathrm{M}$ (average): 2250.4 , found 2250.4 .

\subsection{Synthesis of SEA ${ }^{\text {off }}$ peptide segments for cyclization studies (Figure 5)}

\section{Synthesis of peptide 9a}

Peptide 9a was synthesized on a $0.2 \mathrm{mmol}$ scale as described in the general procedure. TFA/ $\mathrm{H}_{2} \mathrm{O} / \mathrm{TIS} /$ thiophenol/thioanisole $87.5: 2.5: 5: 2.5: 2.5(20 \mathrm{~mL})$ was used as the cleavage cocktail. Purification of the crude by preparative HPLC (eluent A $0.10 \%$ TFA in water, eluent B $0.10 \%$ TFA in $\mathrm{CH}_{3} \mathrm{CN} /$ water: $4 / 1$ by vol, $0-10 \%$ eluent $\mathrm{B}$ in 2 min then $10-30 \%$ in 40 min, $25{ }^{\circ} \mathrm{C}$, detection at $215 \mathrm{~nm}, 51 \mathrm{~mL} / \mathrm{min}, \mathrm{C} 18$ Xbridge $5 \mu \mathrm{m}(19 \times 100 \mathrm{~mm})$ column $)$ provided the titled peptide as a white solid after lyophilisation (146 mg, 30\%). 
Characterization of peptide 9 a:

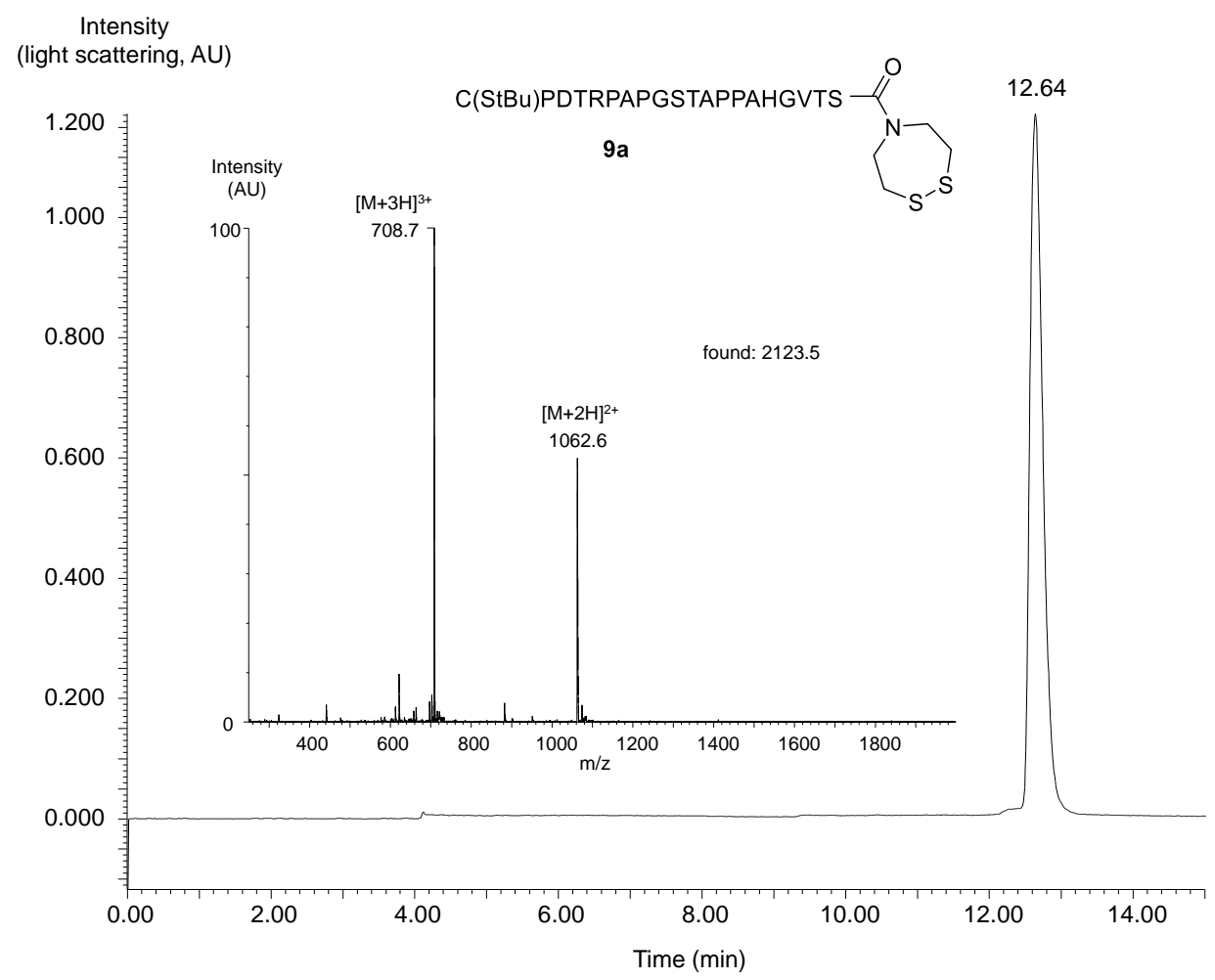

Figure S 36. LC-MS analysis of peptide 9a. LC trace: eluent A $0.10 \%$ TFA in water, eluent B $0.10 \%$ TFA in $\mathrm{CH}_{3} \mathrm{CN} /$ water: $4 / 1$ by vol. $\mathrm{C} 18$ column, gradient $0-50 \% \mathrm{~B}$ in $15 \mathrm{~min}, 30^{\circ} \mathrm{C}, 1$ $\mathrm{mL} / \mathrm{min}$, light scattering detection. MS trace: $\mathrm{m} / \mathrm{z}=1062.5\left([\mathrm{M}+2 \mathrm{H}]^{2+}\right), 708.7\left([\mathrm{M}+3 \mathrm{H}]^{3+}\right)$. Calcd. for M (average): 2124.5, found: 2123.5.

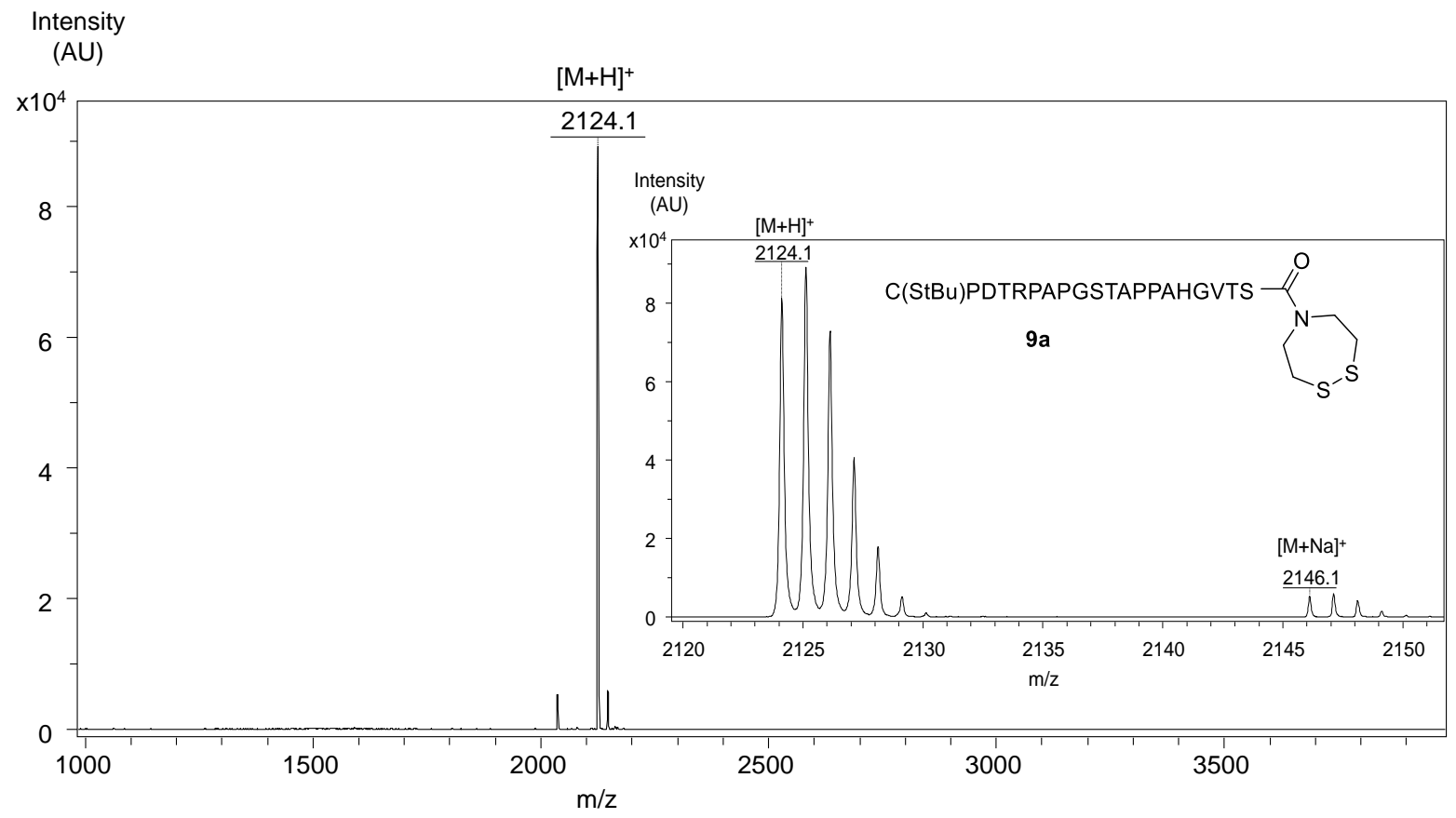

Figure S 37. MALDI-TOF analysis of peptide 9a. Matrix $\alpha$-cyano-4-hydroxycinnamic acid, positive detection mode, calcd. for $[\mathrm{M}+\mathrm{H}]^{+}$(monoisotopic): 2123.94, found: 2124.1. 


\section{Synthesis of peptide $9 \mathrm{~b}$ (linear $\mathrm{K} 1$ precursor)}

The linear K1 precursor $\mathbf{9 b}$ used for the synthesis of $\mathrm{K} 1$ cyclic derivatives was prepared as described in Figure S 38. Its sequence corresponds to HGF 128-205 using Uniprot numbering of amino acid residues (P14210).

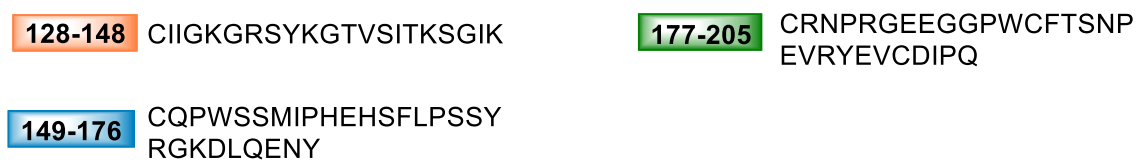

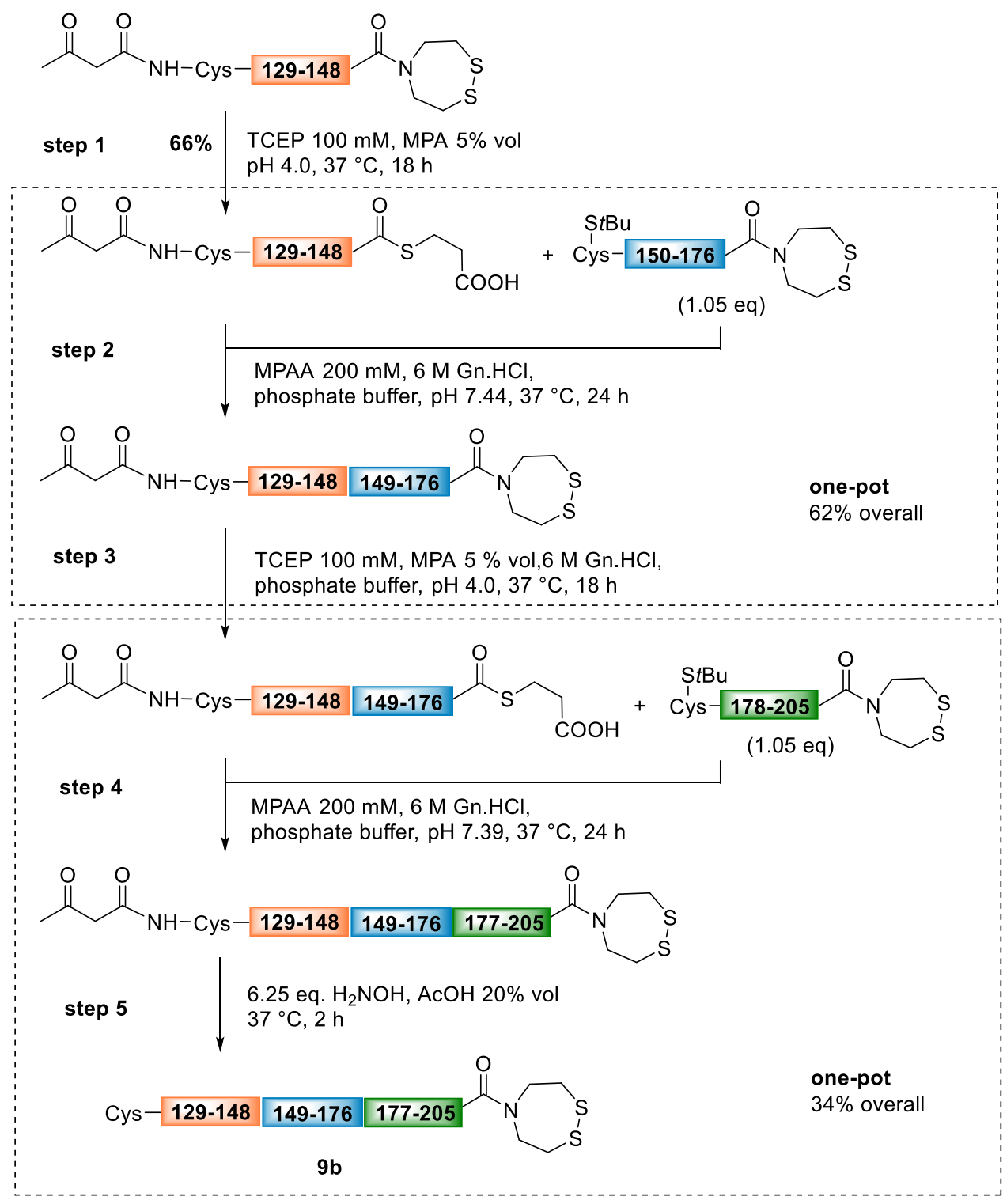

Figure S 38. Assembly of linear K1 precursor $\mathbf{9 b}$. 
AcA-K1[128-148]-SEA ${ }^{\text {off }}{ }^{6}$ and K1[149-176]-SEA ${ }^{\text {off }}$ Erreur ! Signet non défini. have already b een synthesized and characterized elsewhere.

\section{K1[177-205]-SEA ${ }^{\text {off }}$ (C(StBu)RNPRGEEGGPWC(S $t$ Bu)FTSNPEVRYEVC $(\mathrm{S} t \mathrm{Bu}) \mathrm{DIPQ}-$} SEA $^{\text {off }}$ ) was synthesized on a $1.80 \mathrm{mmol}$ scale as described in the general procedure. TFA/ $\mathrm{H}_{2} \mathrm{O} / \mathrm{TIS} /$ thioanisole/EDT 90:1.67:5:1.67:1.67 was used as cleavage cocktail. Purification of the crude by preparative HPLC (eluent A $0.10 \%$ TFA in water, eluent B $0.10 \%$ TFA in $\mathrm{CH}_{3} \mathrm{CN} /$ water: $4 / 1$ by vol, $25{ }^{\circ} \mathrm{C}$, detection at $215 \mathrm{~nm}, 51 \mathrm{~mL} / \mathrm{min}, 0-28 \%$ eluent $\mathrm{B}$ in $5 \mathrm{~min}$ then $28-42 \%$ in $25 \mathrm{~min}, \mathrm{C} 18$ Xbridge $5 \mu \mathrm{m}(19 \times 100 \mathrm{~mm})$ column $)$ provided the titled peptide as a white solid after lyophilisation (404 $\mathrm{mg}, 5 \%)$.

\section{Characterization of K1[177-205]-SEA ${ }^{\text {off }}$ :}

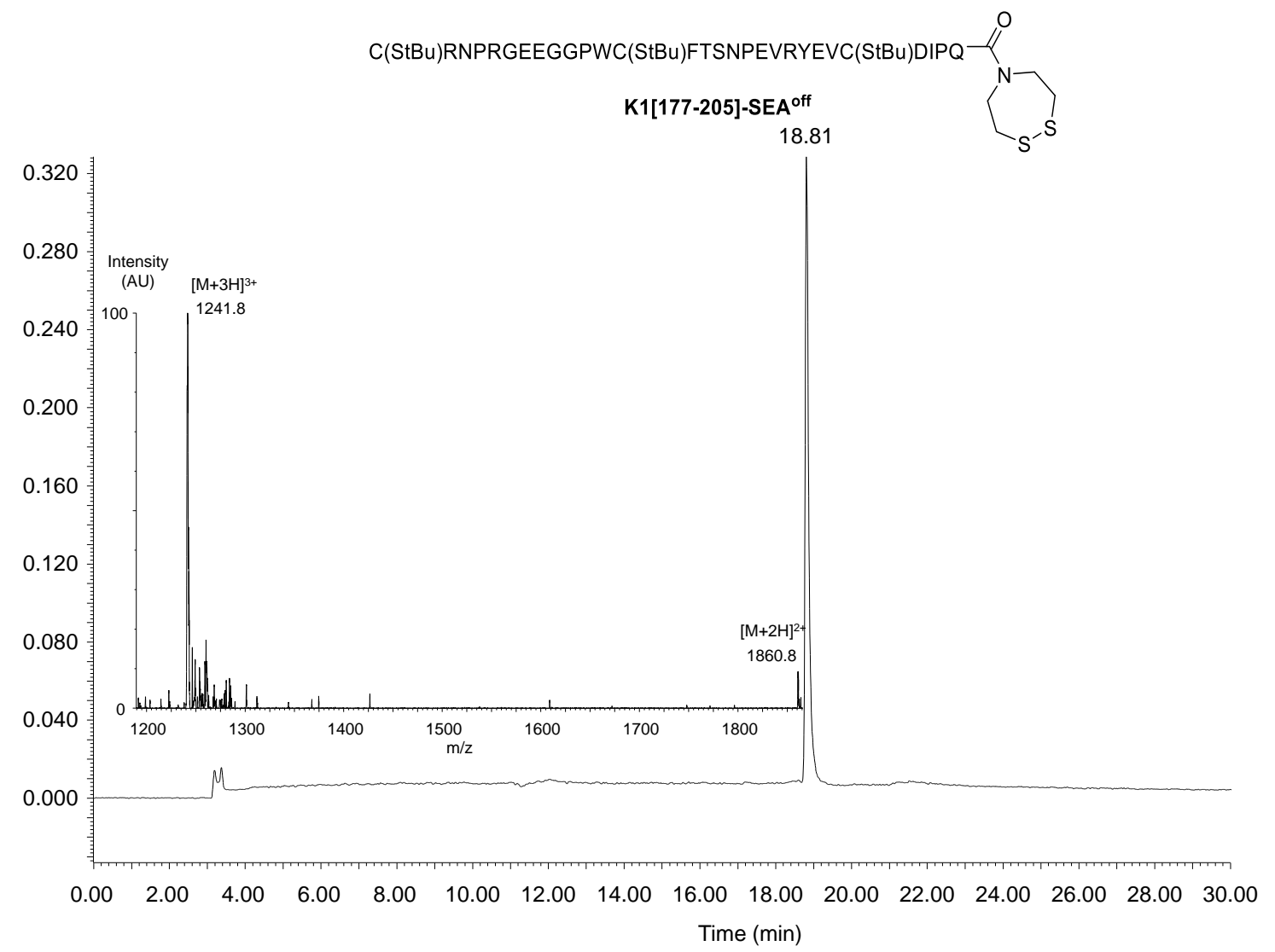

Figure S 39. LC-MS analysis of K1[177-205]-SEA ${ }^{\text {off }}$. LC trace: eluent A $0.10 \%$ TFA in water, eluent $\mathrm{B} 0.10 \%$ TFA in $\mathrm{CH}_{3} \mathrm{CN} /$ water: $4 / 1$ by vol. $\mathrm{C} 18$ column, gradient $0-100 \%$ B in $30 \mathrm{~min}$, $30{ }^{\circ} \mathrm{C}, 1 \mathrm{~mL} / \mathrm{min}$, light scattering detection. MS trace: $\mathrm{m} / \mathrm{z}=1860.8\left([\mathrm{M}+2 \mathrm{H}]^{2+}\right), 1241.8$ $\left([\mathrm{M}+3 \mathrm{H}]^{3+}\right)$; calcd. for M: 3721.4, found: 3721.0. 


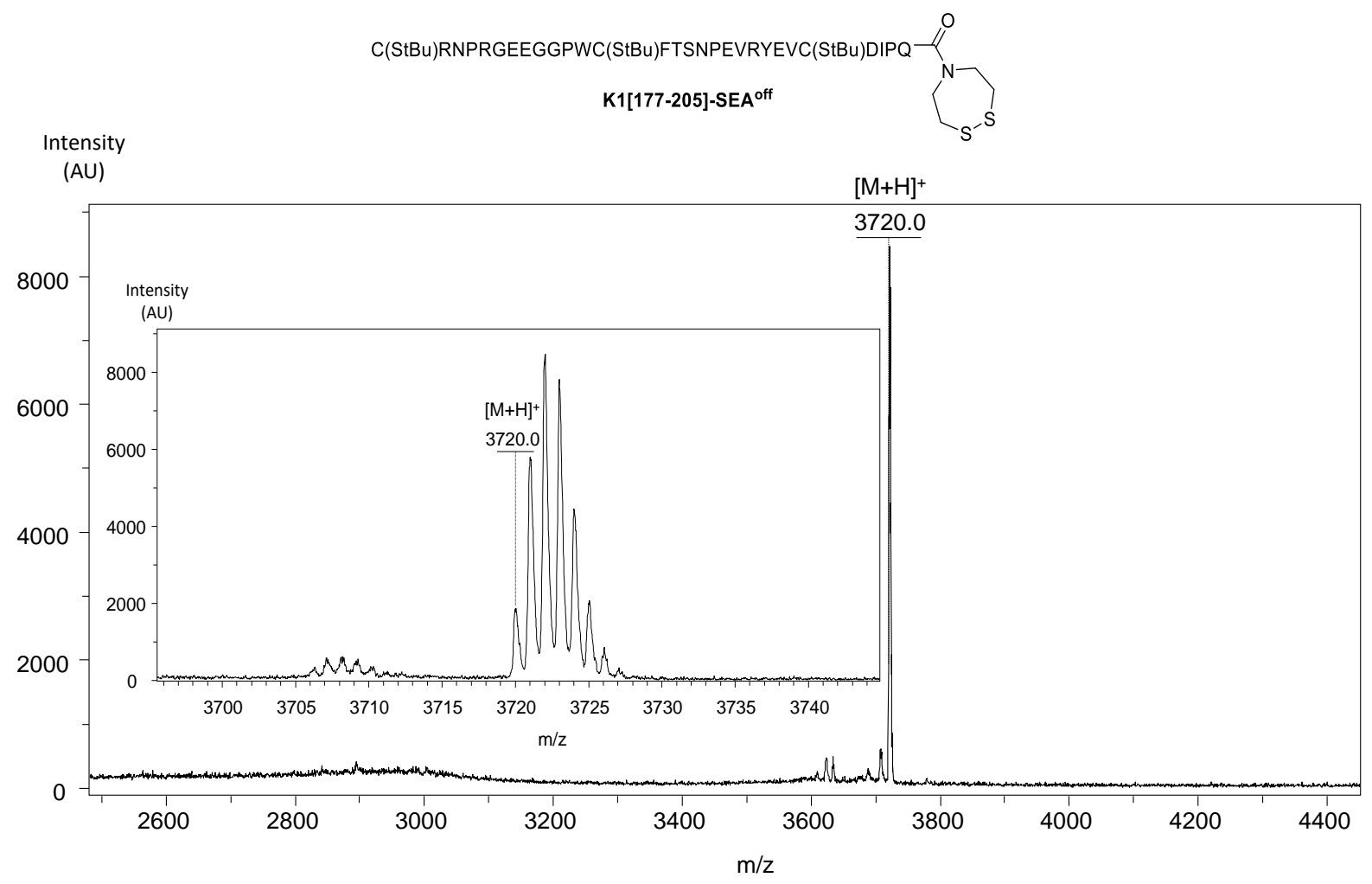

Figure $S$ 40. MALDI-TOF analysis of K1[177-205]-SEA ${ }^{\text {off }}$. Matrix $\alpha$-cyano-4hydroxycinnamic acid, positive detection mode, calcd. for $[\mathrm{M}+\mathrm{H}]^{+}$(monoisotopic) 3719.58, found 3720.0 .

\section{Assembly of linear K1 peptide 9b}

Step 1. Synthesis of peptide thioester AcA-K1[128-148]-MPA

TCEP. $\mathrm{HCl}(286 \mathrm{mg})$ was dissolved in a mixture of MPA $(0.5 \mathrm{~mL})$ and $\mathrm{H}_{2} \mathrm{O}(9.5 \mathrm{~mL})$ and the $\mathrm{pH}$ was adjusted to 4.0 by addition of $6 \mathrm{M} \mathrm{NaOH}$. AcA-K1[128-148]-SEA ${ }^{\text {off }}(23.1 \mathrm{mg}, 7.79$ $\mu \mathrm{mol})$ was added to the solution $(7.78 \mathrm{~mL})$ and the mixture was stirred at $37{ }^{\circ} \mathrm{C}$ for $18 \mathrm{~h}$. Note that a white solid precipitated during the reaction. The addition of $\mathrm{Gn} \cdot \mathrm{HCl}(7.35 \mathrm{~g}$, final concentration: $6 \mathrm{M})$ and MPA $(0.255 \mathrm{~mL}$, final concentration: $5 \% \mathrm{v} / \mathrm{v})$ did not allow the dissolution of the precipitate. The reaction mixture was acidified with $\mathrm{AcOH}(1.2 \mathrm{~mL})$ and extracted with $\mathrm{Et}_{2} \mathrm{O}(3 \times)$ to remove the MPA. The crude was purified by HPLC (eluent $\mathrm{A}=$ water containing $0.1 \% \mathrm{TFA}$, eluent $\mathrm{B}=$ acetonitrile in water $4 / 1$ containing $0.1 \% \mathrm{TFA}, 30^{\circ} \mathrm{C}$, detection at $215 \mathrm{~nm}, 6 \mathrm{~mL} / \mathrm{min}, 0-15 \%$ eluent $\mathrm{B}$ in $5 \mathrm{~min}$, then $15-35 \%$ eluent $\mathrm{B}$ in $60 \mathrm{~min}$, C18XBridge column) to give AcA-K1[128-148]-MPA as a with solid (15.1 mg, 66\%). 
Characterization of AcA-K1[128-148]-MPA:

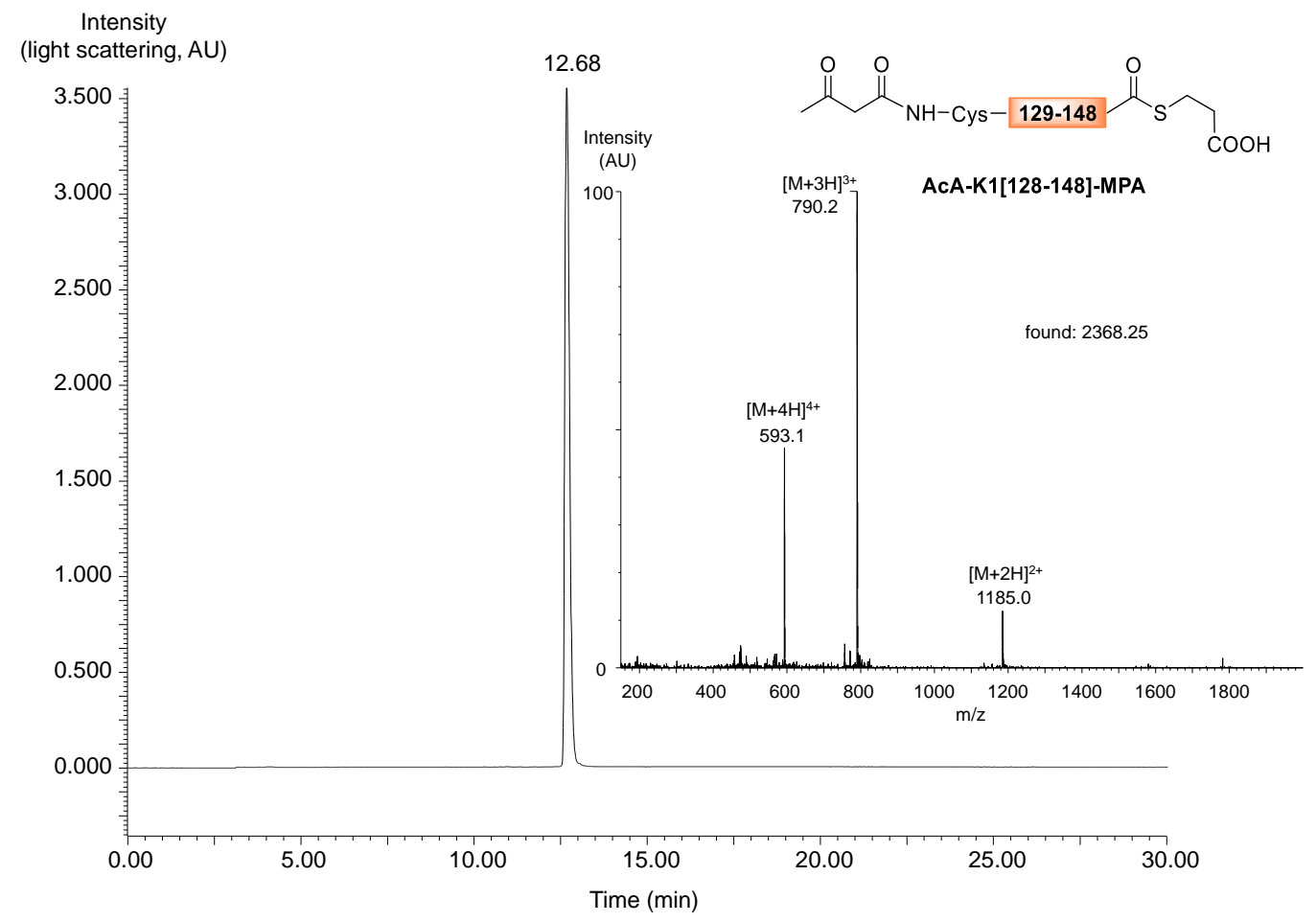

Figure S 41. LC-MS analysis of AcA-K1[128-148]-MPA. LC trace: eluent A 0.10\% TFA in water, eluent B $0.10 \%$ TFA in $\mathrm{CH}_{3} \mathrm{CN} /$ water: 4/1 by vol. $\mathrm{C} 18$ Xbridge $\mathrm{BEH} 300 \AA 5 \mu \mathrm{m}(4.6$ $\times 250 \mathrm{~mm}$ ) column, $30{ }^{\circ} \mathrm{C}$, gradient $0-100 \% \mathrm{~B}$ in $30 \mathrm{~min}, 1 \mathrm{~mL} / \mathrm{min}$, light scattering detection. MS trace: $\mathrm{m} / \mathrm{z}=1185.0\left([\mathrm{M}+2 \mathrm{H}]^{2+}\right), 790.2\left([\mathrm{M}+3 \mathrm{H}]^{3+}\right), 593.1\left([\mathrm{M}+4 \mathrm{H}]^{4+}\right)$; calcd. for $\mathrm{M}$ (average): 2368.8, found: 2368.2.

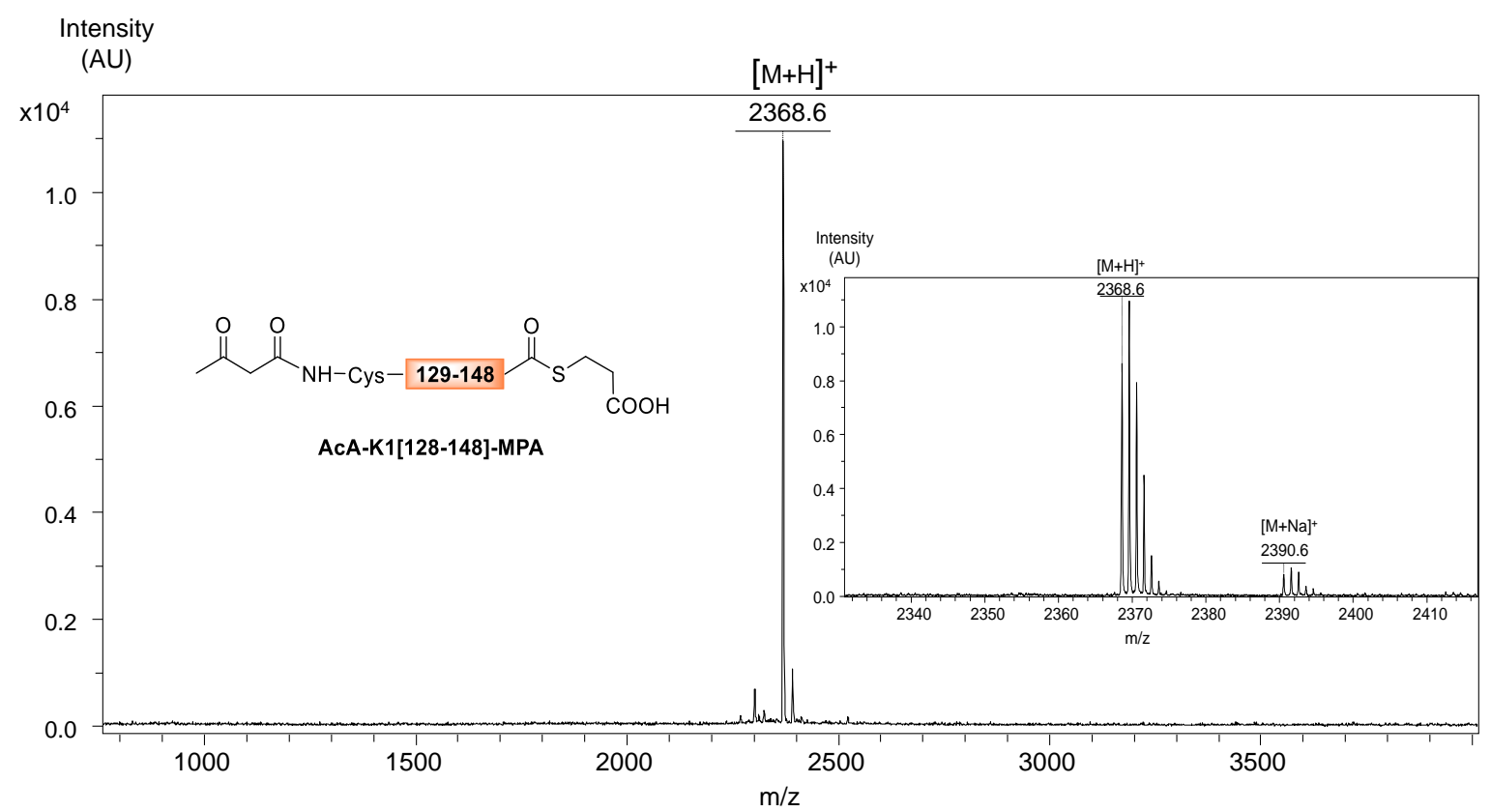

Figure S 42. MALDI-TOF analysis of AcA-K1[128-148]-MPA. Matrix 2,5-dihydroxybenzoic acid (DHB), positive detection mode, calcd. for $[\mathrm{M}+\mathrm{H}]^{+}$(monoisotopic) 2368.27, found 2368.6. 
Step 2-3. Synthesis of peptide thioester AcA-K1[128-176]-MPA

To a solution of $\mathrm{Gn} \cdot \mathrm{HCl}(574 \mathrm{mg})$ in $0.1 \mathrm{M}$, pH 7.4 phosphate buffer $(600 \mu \mathrm{L})$ was added MPAA (33.4 mg, $200 \mathrm{mM}$ ) and the $\mathrm{pH}$ of the mixture was adjusted to 7.44 by addition of $6 \mathrm{M}$ $\mathrm{NaOH}(80 \mu \mathrm{L})$. K1[149-176]- SEA ${ }^{\text {off }}(21.5 \mathrm{mg}, 5.22 \mu \mathrm{mol}, 1.05 \mathrm{eq})$ and AcA-K1[128-148]MPA (14.6 mg, $4.97 \mu \mathrm{mol}, 1 \mathrm{eq})$ were then successively dissolved in the MPAA solution (710 $\mu \mathrm{L})$ and the mixture was stirred at $37^{\circ} \mathrm{C}$. The progress of the reaction leading to peptide AcAK1[128-176]-SEA ${ }^{\text {off }}$ was followed by HPLC.

After completion of the NCL ligation (24h), SEA peptide AcA-K1[128-176]-SEA ${ }^{\text {off }}$ was directly converted into the corresponding MPA thioester by addition MPA (5\% v/v) and TCEP (100 mM). To perform this reaction, TCEP $(222 \mathrm{mg}, 111 \mathrm{mM})$ and MPA $(388 \mu \mathrm{L}, 5.55 \% \mathrm{v} / \mathrm{v})$ were added to the solution of $\mathrm{Gn} \cdot \mathrm{HCl}(4.01 \mathrm{~g})$ in $0.1 \mathrm{M}, \mathrm{pH} 7.4$ phosphate buffer $(3.81 \mathrm{~mL})$ and the $\mathrm{pH}$ of the mixture was adjusted to 4.00 by addition of $6 \mathrm{M} \mathrm{NaOH}(600 \mu \mathrm{L})$. The previous ligation mixture containing AcA-K1[128-176]-SEA ${ }^{\text {off }}$ was then diluted with the MPA/TCEP solution $(6.38 \mathrm{~mL})$ and the $\mathrm{pH}$ was readjusted to 4.00 by addition of $6 \mathrm{~N} \mathrm{HCl}(25 \mu \mathrm{L})$. In these conditions $([\mathrm{TCEP}]=100 \mathrm{mM}, 5 \% \mathrm{MPA} \mathrm{v} / \mathrm{v})$, the reaction mixture was stirred at $37^{\circ} \mathrm{C}$ for 18 h. After completion of the reaction, the mixture was acidified with $\mathrm{AcOH}(0.7 \mathrm{~mL})$ and extracted with $\mathrm{Et}_{2} \mathrm{O}(5 \times)$ to remove the MPA and the MPAA. The crude was purified by HPLC (eluent $\mathrm{A}=$ water containing $0.1 \%$ TFA, eluent $\mathrm{B}=$ acetonitrile in water $4 / 1$ containing $0.1 \%$ TFA, $50^{\circ} \mathrm{C}$, detection at $215 \mathrm{~nm}, 6 \mathrm{~mL} / \mathrm{min}, 0-20 \%$ eluent B in $5 \mathrm{~min}$, then 20-40\% eluent B in 60 min, C18XBridge column) to give AcA-K1[128-176]-MPA as a with solid after lyophilisation $(20.8 \mathrm{mg}, 62 \%)$.

Characterization of AcA-K1[128-176]-MPA:

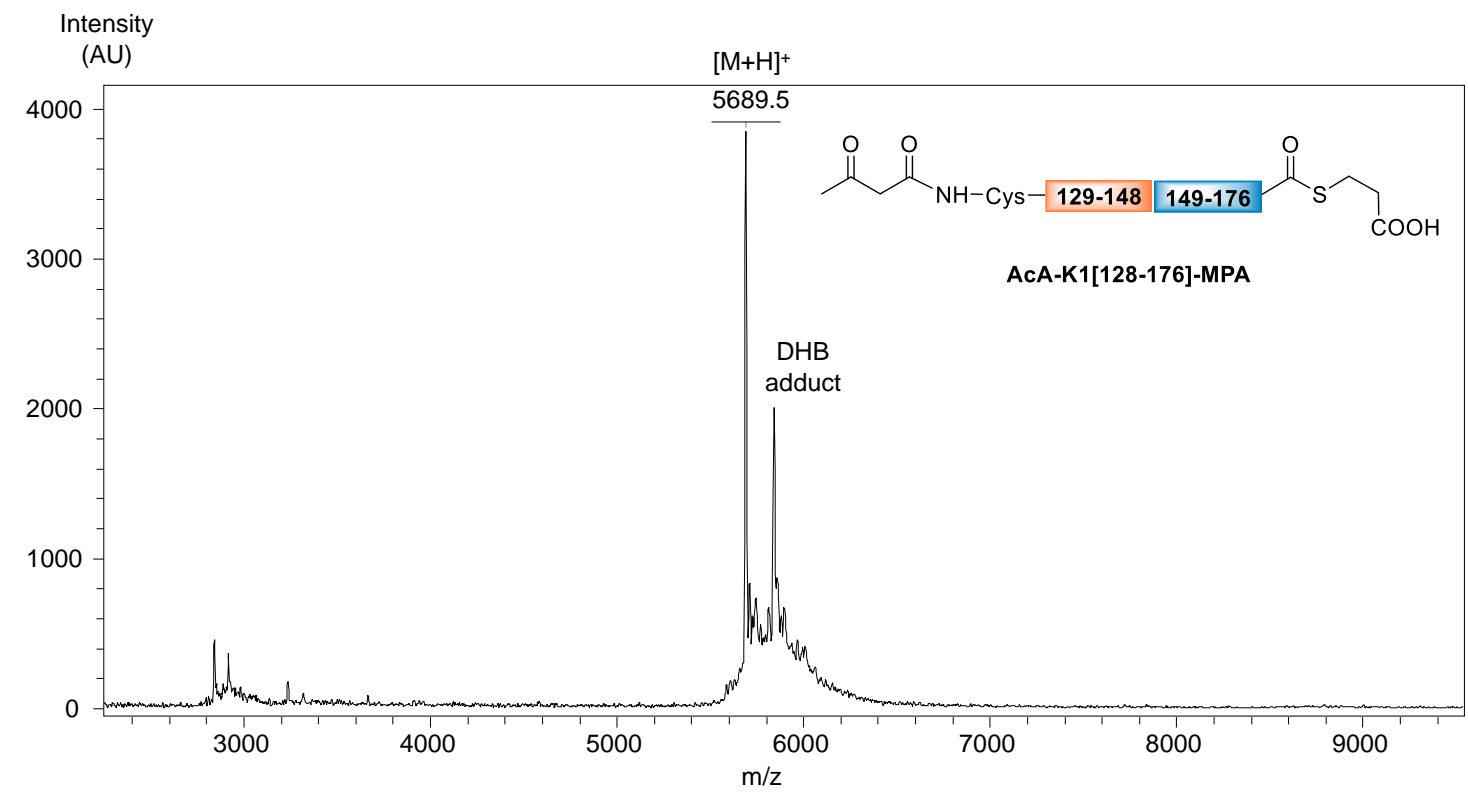

Figure S 43. MALDI-TOF analysis of AcA-K1[128-176]-MPA. Matrix 2,5-dihydroxybenzoic acid (DHB), positive detection mode, calcd. for $[\mathrm{M}+\mathrm{H}]^{+}$(average) 5689.52, found 5689.5. 


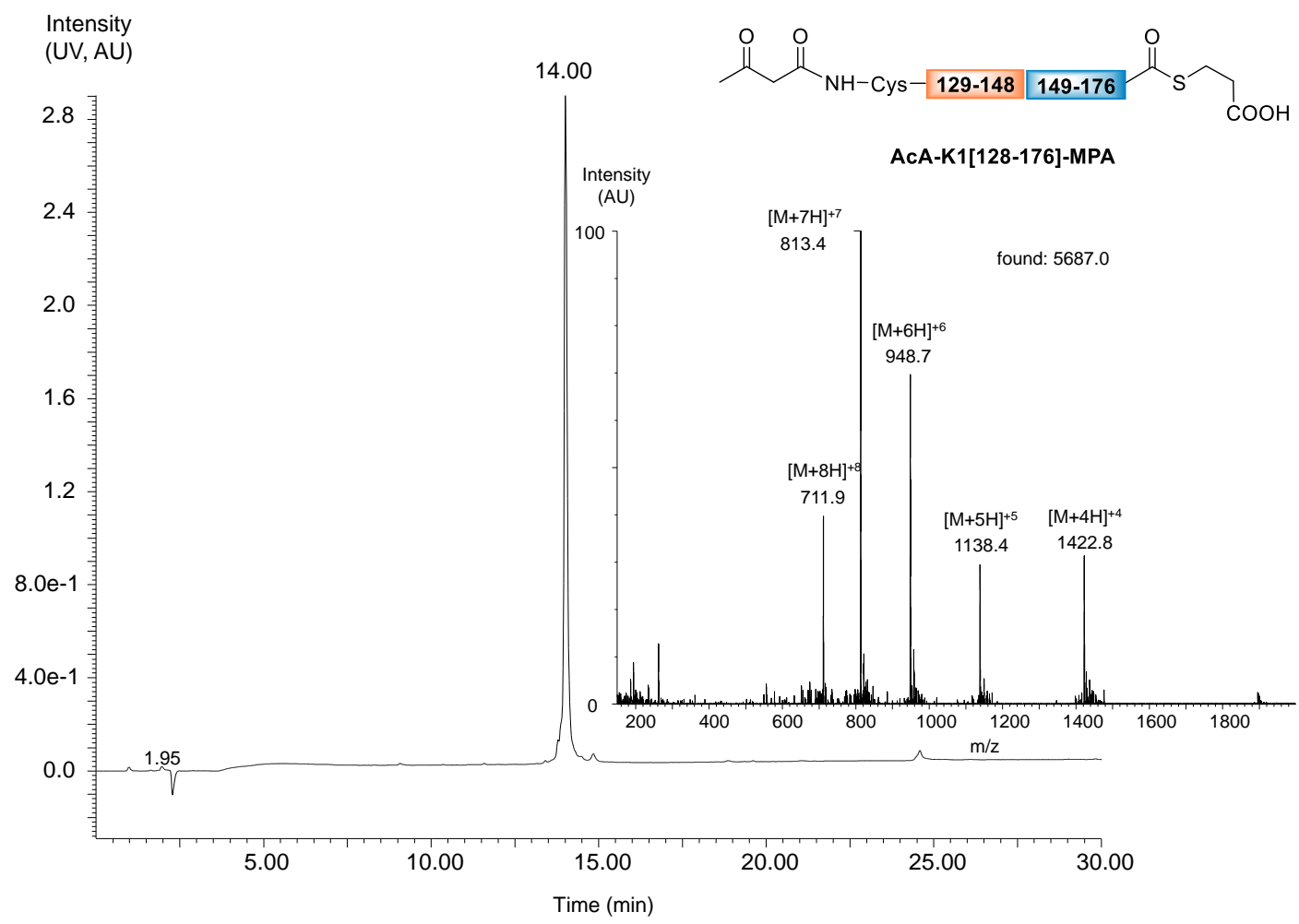

Figure S 44. LC-MS analysis of AcA-K1[128-176]-MPA. LC trace: eluent A 0.10\% TFA in water, eluent $\mathrm{B} 0.10 \%$ TFA in $\mathrm{CH}_{3} \mathrm{CN} /$ water: $4 / 1$ by vol. $\mathrm{C} 18$ Xbridge $\mathrm{BEH} 300 \AA 5 \mu \mathrm{m}(4.6$ $\times 250 \mathrm{~mm}$ ) column, $50{ }^{\circ} \mathrm{C}$, gradient $0-100 \% \mathrm{~B}$ in $30 \mathrm{~min}, 1 \mathrm{~mL} / \mathrm{min}$, UV detection. MS trace: $\mathrm{m} / \mathrm{z}=1422.8\left([\mathrm{M}+4 \mathrm{H}]^{4+}\right), 1138.4\left([\mathrm{M}+5 \mathrm{H}]^{5+}\right), 948.7\left([\mathrm{M}+6 \mathrm{H}]^{6+}\right), 813.4\left([\mathrm{M}+7 \mathrm{H}]^{7+}\right), 711.9$ $\left([\mathrm{M}+8 \mathrm{H}]^{8+}\right)$; calcd. for M: 5688.5, found: 5687.0.

\section{Step 4-5. Synthesis of peptide K1[128-205]-SEA ${ }^{\text {off }}(\mathbf{9 b})$}

To a solution of $\mathrm{Gn} \cdot \mathrm{HCl}(574 \mathrm{mg})$ in $0.1 \mathrm{M}, \mathrm{pH} 7.4$ phosphate buffer $(600 \mu \mathrm{L})$ was added MPAA $(33.7 \mathrm{mg}$ ) and the $\mathrm{pH}$ of the mixture was adjusted to 7.39 by addition of $6 \mathrm{M} \mathrm{NaOH}(75$ $\mu \mathrm{L})$. K1[177-205]-SEA ${ }^{\text {off }}(13.5 \mathrm{mg}, 3.25 \mu \mathrm{mol}, 1.05 \mathrm{eq})$ and AcA-K1[128-176]-MPA (20.8 $\mathrm{mg}, 3.10 \mu \mathrm{mol}, 1 \mathrm{eq})$ were then successively dissolved in the MPAA solution $(442 \mu \mathrm{L})$ and the mixture was stirred at $37^{\circ} \mathrm{C}$. The progress of the reaction leading to peptide AcA-K1[128-205]SEA $^{\text {off }}$ was followed by HPLC.

After completion of the NCL ligation (24h), the AcA protecting group of AcA-K1[128-205]SEA ${ }^{\text {off }}$ was removed by reaction with $\mathrm{NH}_{2} \mathrm{OH}$. To perform this deprotection, the reaction mixture was acidified with $\mathrm{AcOH}(110 \mu \mathrm{L})$ and treated with a $0.71 \mathrm{M}$ solution of $\mathrm{NH}_{2} \mathrm{OH} \cdot \mathrm{HCl}$ in $\mathrm{H}_{2} \mathrm{O}(27.3 \mu \mathrm{L}, 19.4, \mu \mathrm{mol}, 6.25 \mathrm{eq})$. After $2 \mathrm{~h}$ stirring at $37^{\circ} \mathrm{C}$, the reaction mixture was diluted with $\mathrm{H}_{2} \mathrm{O}(570 \mu \mathrm{L})$ and extracted with $\mathrm{Et}_{2} \mathrm{O}(5 \times)$ to remove the MPAA. The crude was further diluted with $\mathrm{H}_{2} \mathrm{O}(1.14 \mathrm{~mL},[\mathrm{AcOH}] \approx 5 \% \mathrm{v} / \mathrm{v})$ and purified by HPLC (eluent $\mathrm{A}=$ water containing $0.1 \%$ TFA, eluent $\mathrm{B}=$ acetonitrile in water $4 / 1$ containing $0.1 \% \mathrm{TFA}, 50^{\circ} \mathrm{C}$, detection at $215 \mathrm{~nm}, 6 \mathrm{~mL} / \mathrm{min}, 0-25 \%$ eluent $\mathrm{B}$ in $5 \mathrm{~min}$, then 25-75\% eluent B in $75 \mathrm{~min}, \mathrm{C} 18 \mathrm{XBridge}$ column) to give K1[128-205]-SEA ${ }^{\text {off }}$ (9b) as a with solid after lyophilisation(10.9 $\mathrm{mg}$, 34\%). 
Characterization of peptide $\mathbf{9 b}$ :

a)

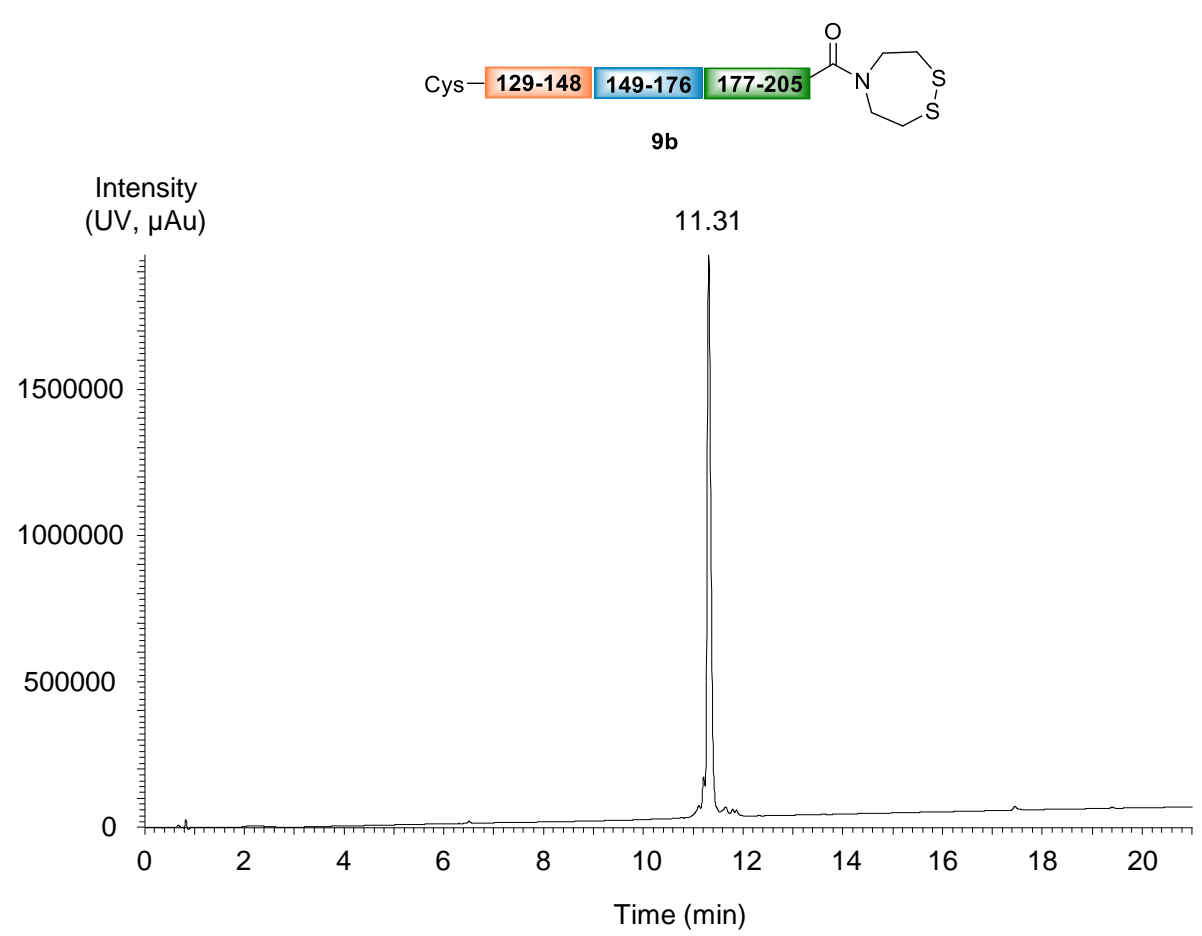

b)

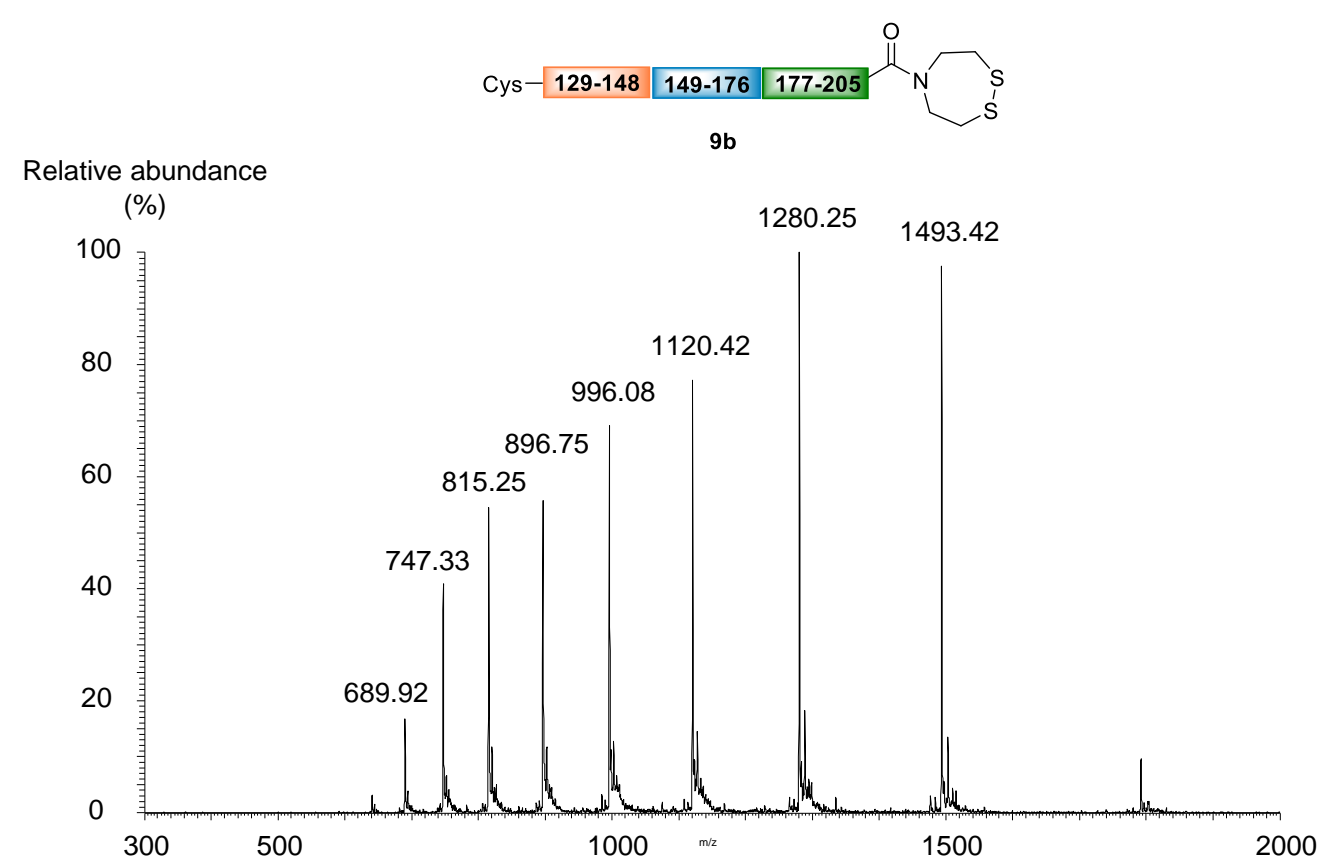

Figure S 45. LC-MS analysis of peptide 9b. a) LC trace. Eluent A 0.10\% TFA in water, eluent B $0.10 \%$ TFA in $\mathrm{CH}_{3} \mathrm{CN}$, ACQUITY UPLC Peptide BEH $300 \AA 1.7 \mu \mathrm{m}(2.1 \times 150 \mathrm{~mm})$ column, $50{ }^{\circ} \mathrm{C}$, gradient $0-70 \% \mathrm{~B}$ in $20 \mathrm{~min}, 0.4 \mathrm{~mL} / \mathrm{min}$, UV detection. b) MS trace: $\mathrm{m} / \mathrm{z}=$ $1493.42\left([\mathrm{M}+6 \mathrm{H}]^{6+}\right), 1280.25\left([\mathrm{M}+7 \mathrm{H}]^{7+}\right), 1120.42\left([\mathrm{M}+8 \mathrm{H}]^{8+}\right), 996.08\left([\mathrm{M}+9 \mathrm{H}]^{9+}\right), 896.75$ $\left([\mathrm{M}+10 \mathrm{H}]^{10+}\right), 815.25\left([\mathrm{M}+11 \mathrm{H}]^{11+}\right), 747.33\left([\mathrm{M}+12 \mathrm{H}]^{12+}\right), 689.92\left([\mathrm{M}+13 \mathrm{H}]^{13+}\right)$; calcd. for $\mathrm{M}$ (average): 8955.26, found: 8955.81. 


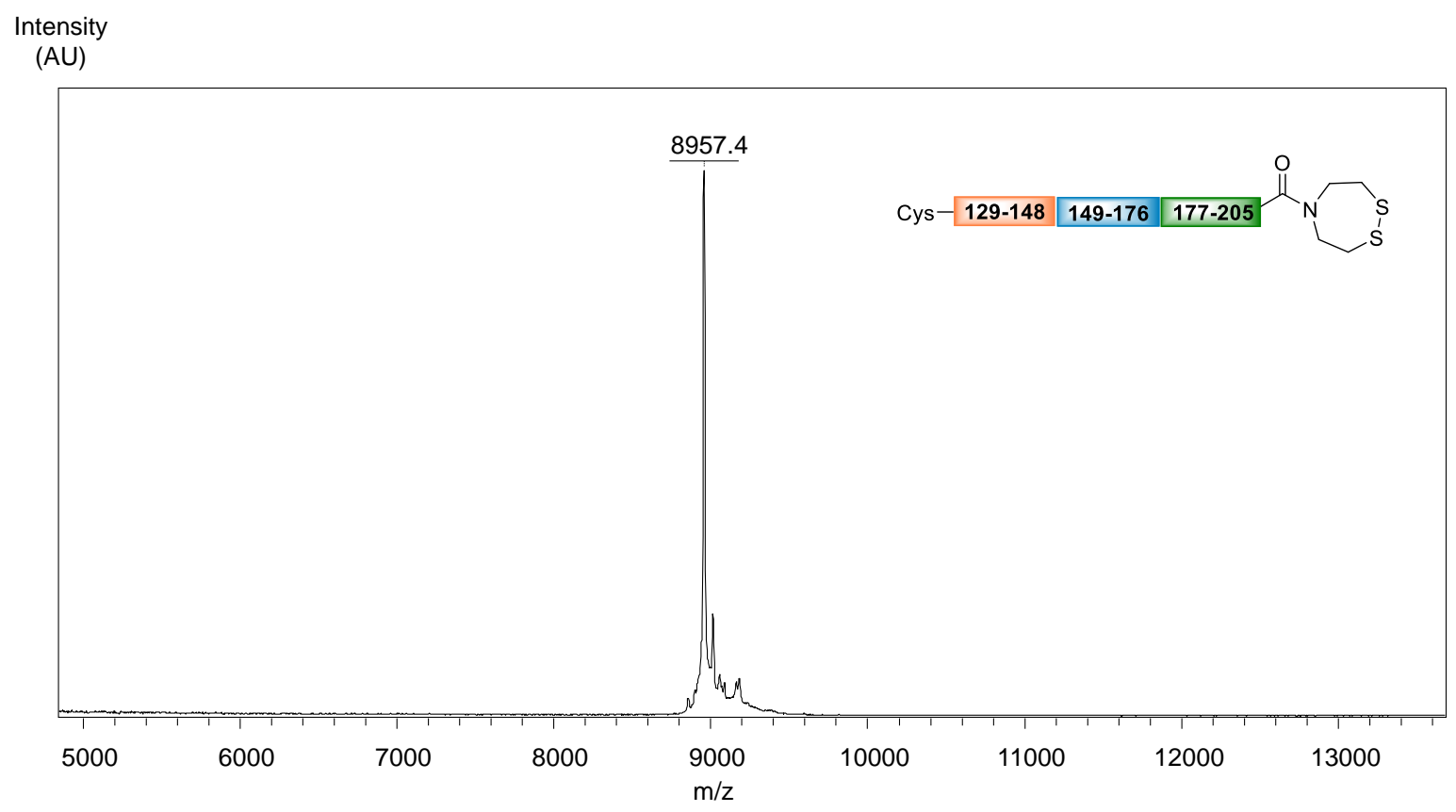

Figure S 46. MALDI-TOF analysis of peptide 9b. Matrix 2,5-dihydroxybenzoic acid (DHB), positive detection mode, calcd. for $[\mathrm{M}+\mathrm{H}]^{+}$(average) 8956.22, found: 8957.4. 


\section{Stability and reactivity of SetCys unit as a function of the reducing power of the reaction mixture (Figure 2a)}

\subsection{Stability of SetCys under low (property 1) or strong (property 4) reducing conditions}

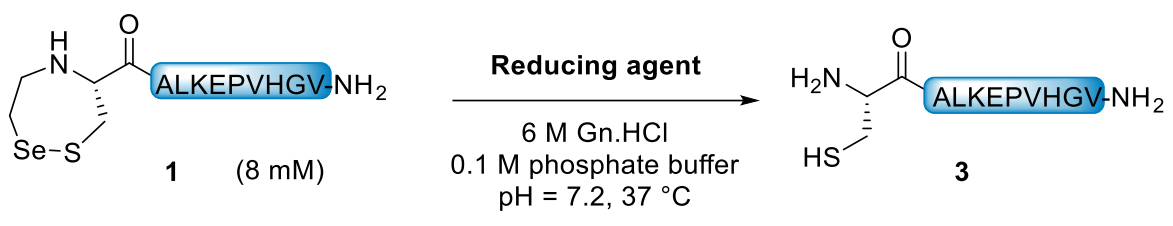

Reducing agent: MPAA, MPAA + DTT, MPAA + TCEP

Figure S 47: Stability of the SetCys unit as a function of the reducing power of the reaction mixture.

General procedure illustrated for property 4 using MPAA + TCEP:

$8 \mathrm{mM}$ peptide, $200 \mathrm{mM}$ MPAA, $100 \mathrm{mM}$ TCEP, $100 \mathrm{mM}$ ascorbate, $\mathrm{pH} 7.2$ in $0.1 \mathrm{M}$ phosphate buffer

To a solution of $\mathrm{Gn} \cdot \mathrm{HCl}(287 \mathrm{mg})$ in $0.1 \mathrm{M}, \mathrm{pH} 7.4$ phosphate buffer $(300 \mu \mathrm{L})$ were added TCEP. $\mathrm{HCl}(14.3 \mathrm{mg})$, sodium ascorbate $(9.9 \mathrm{mg})$ and MPAA $(16.8 \mathrm{mg})$ and the $\mathrm{pH}$ of the mixture was adjusted to 7.2 by addition of $6 \mathrm{M} \mathrm{NaOH}$. SetCys peptide 1 ( $0.96 \mathrm{mg}, 0.64 \mu \mathrm{mol})$ was then dissolved in this solution $(80 \mu \mathrm{L})$ and the reaction mixture was stirred at $37{ }^{\circ} \mathrm{C}$. The conversion of SetCys into Cys was monitored by HPLC. For each point, a $2 \mu \mathrm{L}$ aliquot was taken from the reaction mixture and quenched by adding $10 \% \mathrm{AcOH}$ in water $(100 \mu \mathrm{L})$. The sample was then extracted with $\mathrm{Et}_{2} \mathrm{O}$ to remove MPAA prior to HPLC analysis. The kinetic data were then fitted to a pseudo-first order kinetic law using SigmaPlot software.

This reaction was repeated in various reductive conditions (MPAA, MPAA + DTT) and the results of this kinetic study are given in Table $S 1$ and Figure $S$ 48. The monitoring of the reaction by HPLC is shown in Figure S 49, Figure $S 50$ and Figure S 51. 


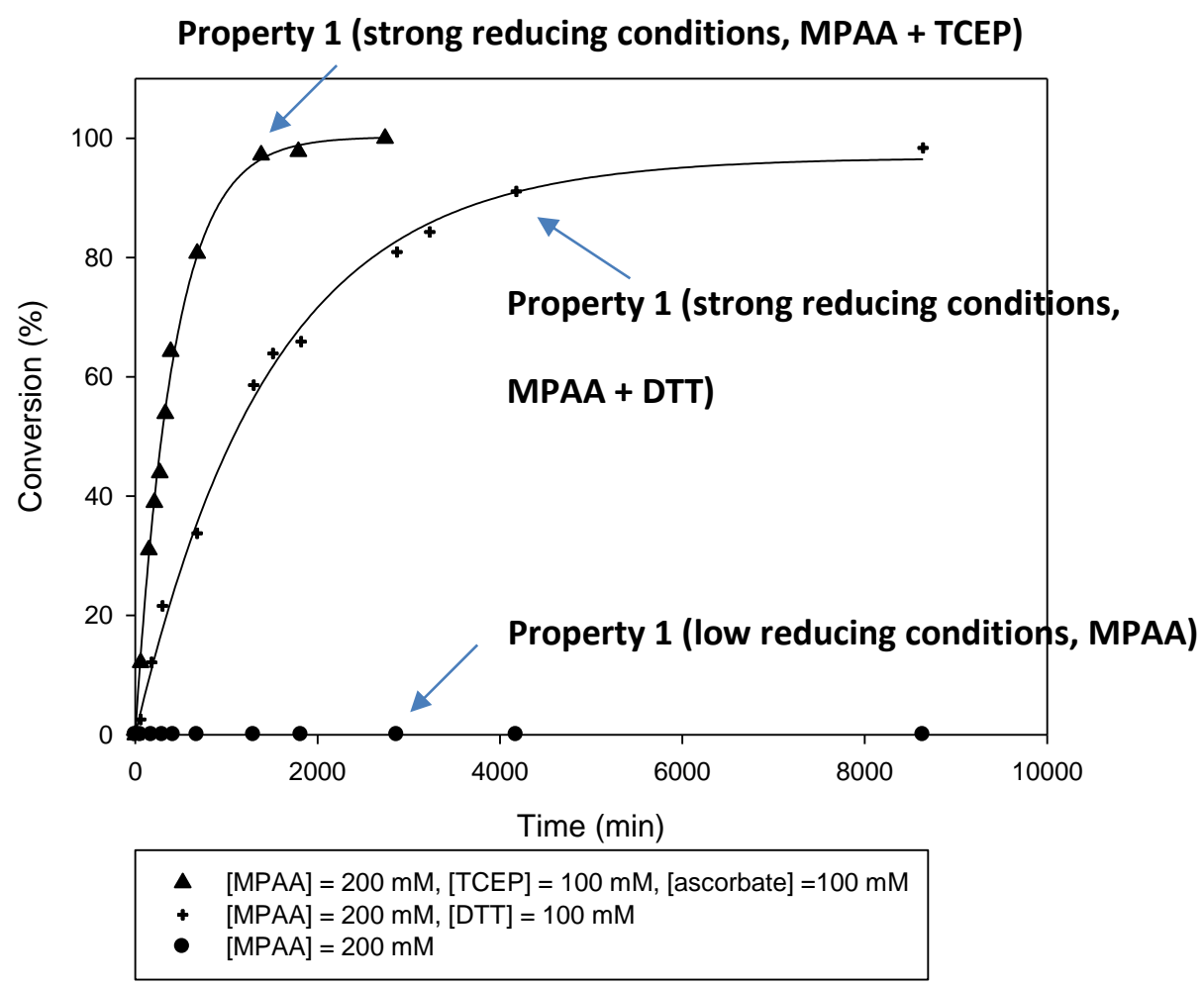

Figure S 48. Conversion of SetCys peptide 1 into Cys peptide 3 in presence of various reducing agents. The continuous curves correspond to the fitting to a pseudo first order kinetic law from which $t^{1} 12$ and $\mathrm{k}_{1}$ were extracted.

Table S 1. Kinetic data extracted from curve fitting in Figure S 48.

\begin{tabular}{cccccc} 
[MPAA] $(\mathbf{m M})$ & {$[\mathbf{D T T}](\mathbf{m M})$} & [TCEP] $(\mathbf{m M})$ & [ascorbate] $(\mathbf{m M})$ & $\mathbf{k}_{\mathbf{1}}\left(\mathbf{m i n}^{-1}\right)$ & $\mathbf{t}_{\mathbf{1} / \mathbf{2}}(\mathbf{h})$ \\
\hline $\mathbf{2 0 0}$ & 0 & 0 & 0 & 0 & 0 \\
200 & $\mathbf{1 0 0}$ & 0 & 0 & 0.0007 & 16.5 \\
200 & 0 & $\mathbf{1 0 0}$ & 100 & 0.0018 & 6.4
\end{tabular}




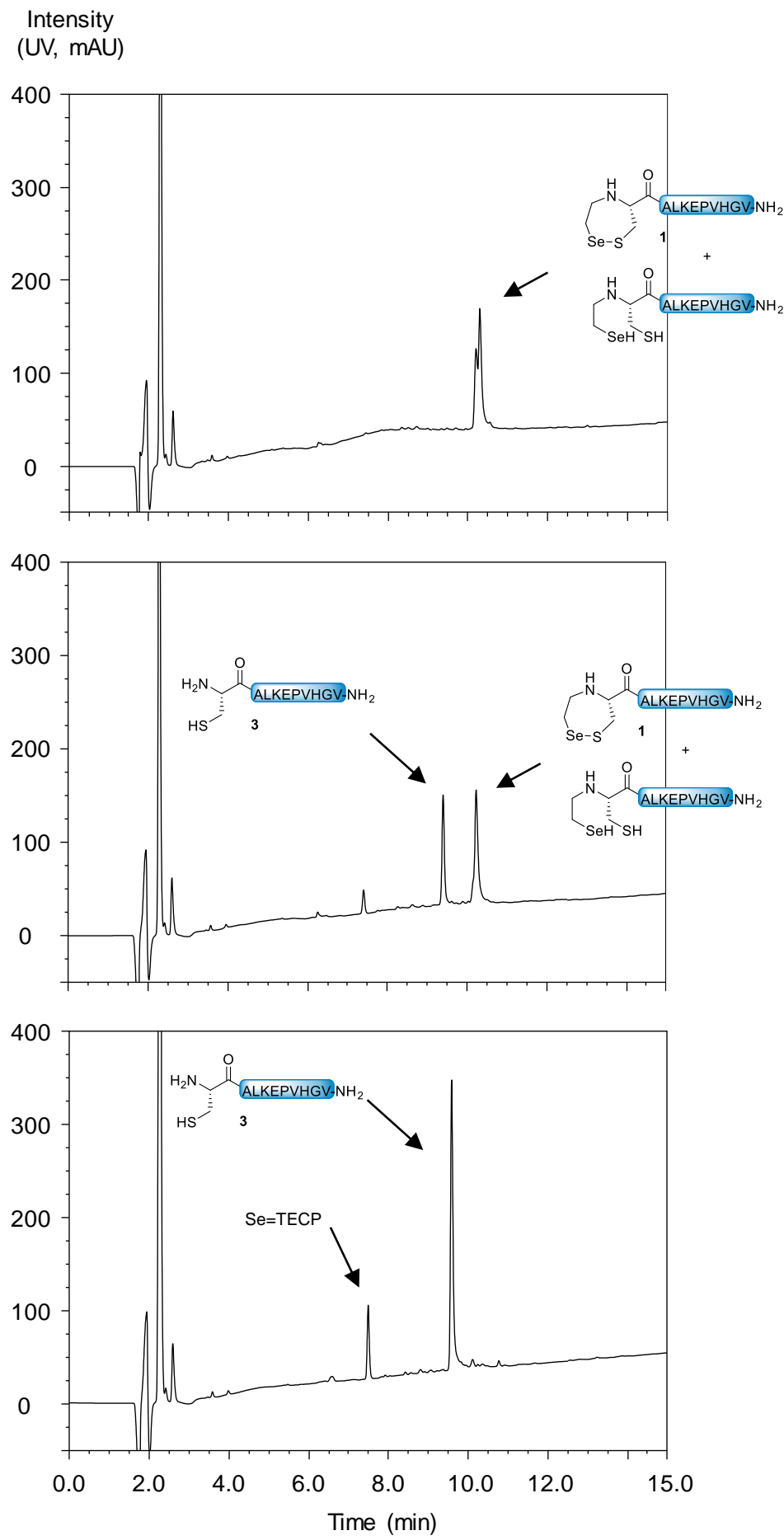

Figure S 49. Monitoring of the conversion of SetCys peptide 1 into cysteinyl peptide 3 using MPAA + TCEP. LC trace: eluent A $0.10 \%$ TFA in water, eluent B $0.10 \%$ TFA in $\mathrm{CH}_{3} \mathrm{CN} /$ water: $4 / 1$ by vol., C18 column, gradient $0-50 \% \mathrm{~B}$ in $15 \mathrm{~min}, 30{ }^{\circ} \mathrm{C}, 1 \mathrm{~mL} / \mathrm{min}$, $\mathrm{UV}$ detection at 215 nm. 


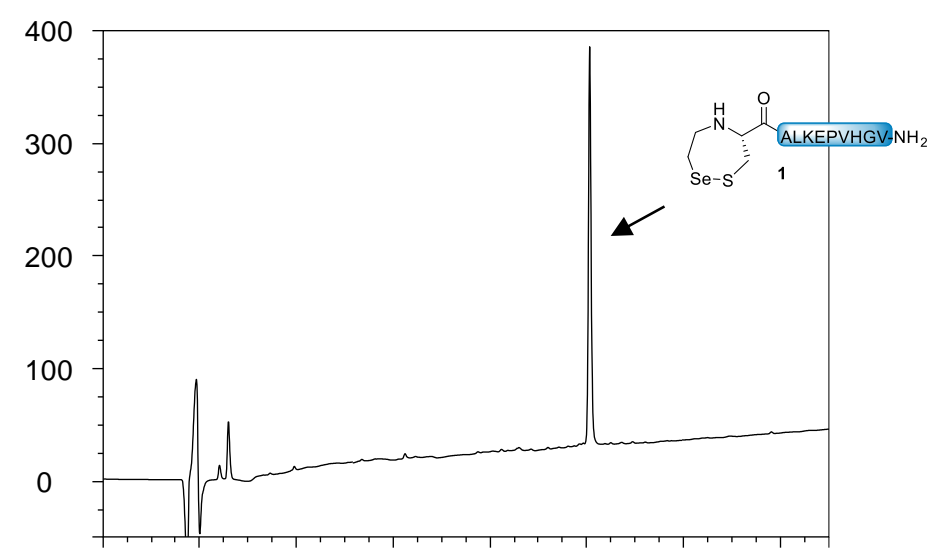

b) $t=25 h$
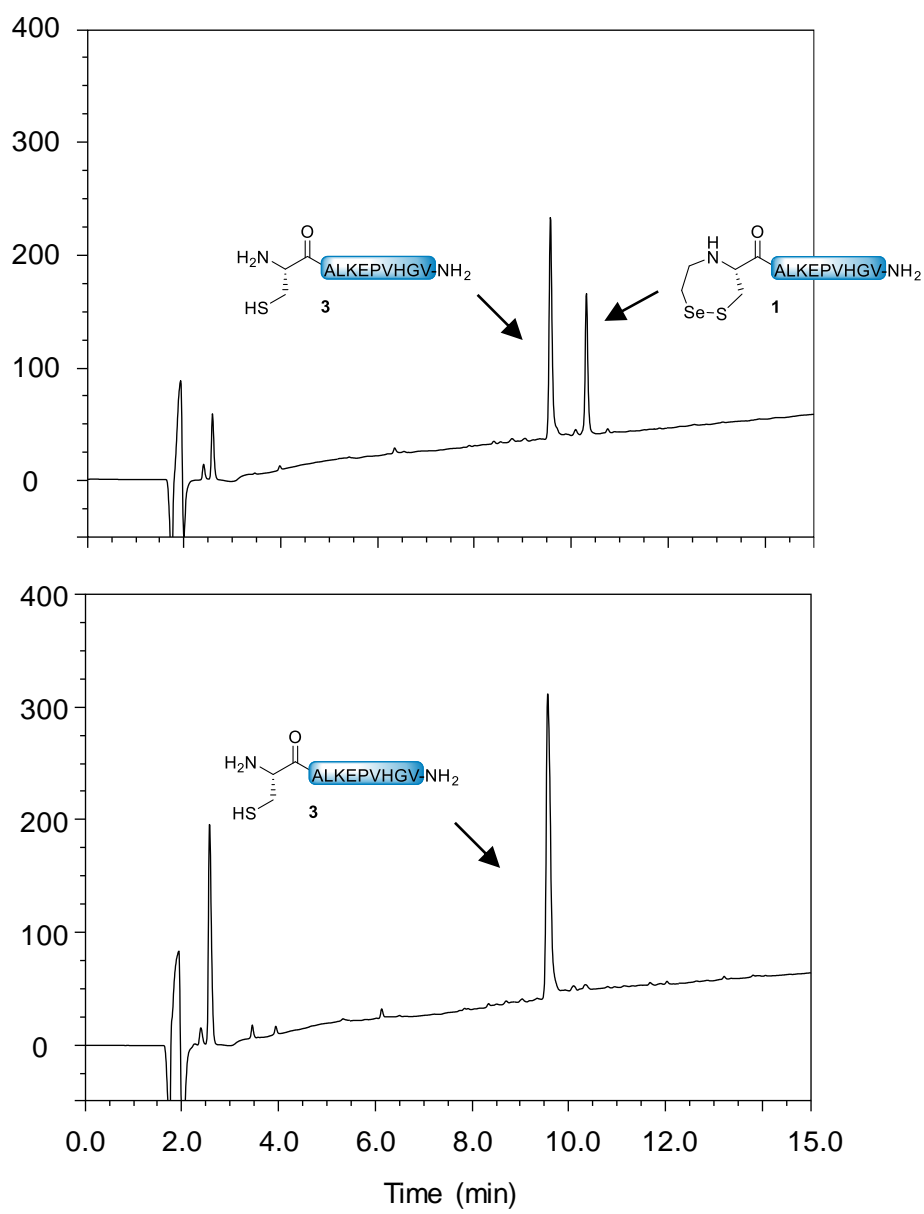

Figure S 50. Monitoring of the conversion of SetCys peptide 1 into cysteinyl peptide 3 using DTT. LC trace: eluent A $0.10 \%$ TFA in water, eluent B $0.10 \%$ TFA in $\mathrm{CH} 3 \mathrm{CN} /$ water: $4 / 1$ by vol., C18 column, gradient $0-50 \%$ B in $15 \mathrm{~min}, 30{ }^{\circ} \mathrm{C}, 1 \mathrm{~mL} / \mathrm{min}$, UV detection at $215 \mathrm{~nm}$. 
a) $\mathrm{t}=20 \mathrm{~s}$
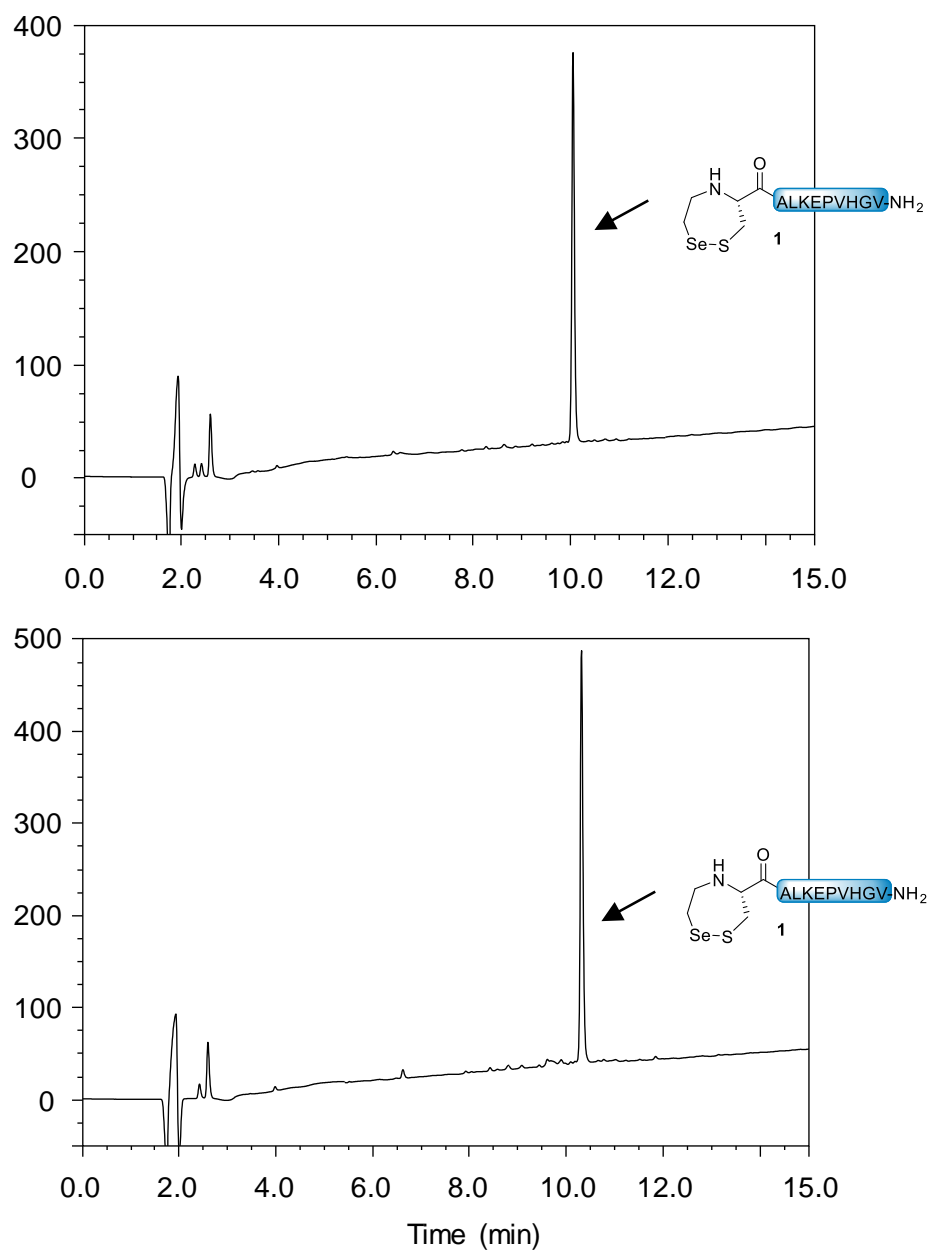

Figure S 51. Stability of the SetCys peptide 1 in the presence of MPAA. LC trace: eluent A $0.10 \%$ TFA in water, eluent $\mathrm{B} 0.10 \%$ TFA in $\mathrm{CH}_{3} \mathrm{CN} /$ water: $4 / 1$ by vol., $\mathrm{C} 18$ column, gradient $0-50 \% \mathrm{~B}$ in $15 \mathrm{~min}, 30{ }^{\circ} \mathrm{C}, 1 \mathrm{~mL} / \mathrm{min}$, UV detection at $215 \mathrm{~nm}$. 


\subsection{Stability of SetCys under strong (property 4) reducing conditions. Importance of ascorbate}
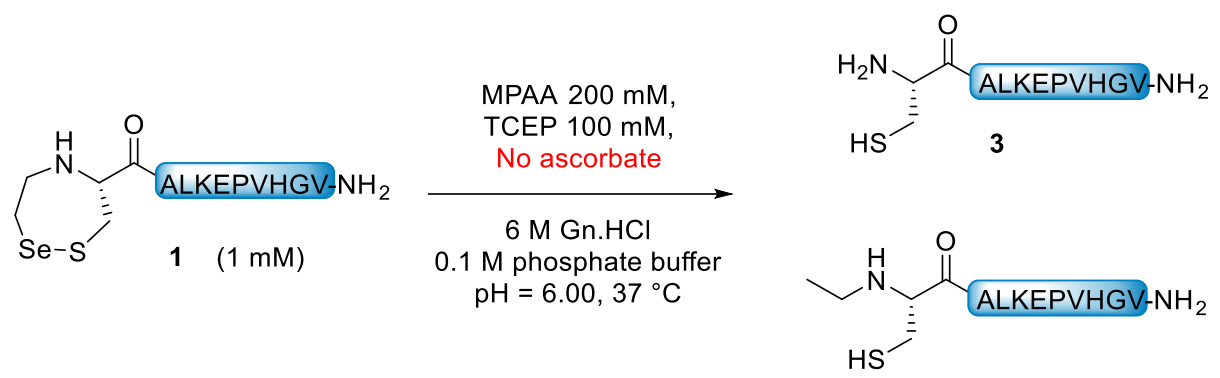

Figure S 52. Treatment of SetCys by TCEP in absence of ascorbate.

To a solution of $\mathrm{Gn} \cdot \mathrm{HCl}(287 \mathrm{mg})$ in $\mathrm{pH} 7.40 .1 \mathrm{M}$ phosphate buffer $(300 \mu \mathrm{L})$ were added TCEP.HCl (14.3 mg) and MPAA $(16.8 \mathrm{mg})$ and the $\mathrm{pH}$ of the mixture was adjusted to 6.0 by addition of $6 \mathrm{M} \mathrm{NaOH}$. SetCys peptide $1(0.48 \mathrm{mg}, 0.32 \mu \mathrm{mol})$ was then dissolved in the buffered solution $(320 \mu \mathrm{L})$ and the reaction mixture was stirred at $37{ }^{\circ} \mathrm{C}$. The progress of the reaction was monitored by HPLC. For each point, an $8 \mu \mathrm{L}$ aliquot was taken from the reaction mixture and quenched by adding $10 \% \mathrm{AcOH}$ in water $(100 \mu \mathrm{L})$. The sample was then extracted with $\mathrm{Et}_{2} \mathrm{O}$ to remove MPAA prior to HPLC analysis.

At pH 6.0 and at the beginning of the reaction, the deselenization of SetCys peptide 1 into the corresponding $N$-ethyl cysteinyl derivative (Et-CALKEPVHGV-NH2) dominates in the mixture (Figure S53a). At longer reaction times, the SetCys peptide 1 also yields the Cys peptide by losing its selenoethyl arm (Figure S 53b). An increase of the reaction mixture $\mathrm{pH}$ (from 6.00 to 7.20) favors the deselenization process and leads nearly exclusively to the formation of the $\mathrm{N}$-ethylcysteinyl peptide product (Figure S 54). 

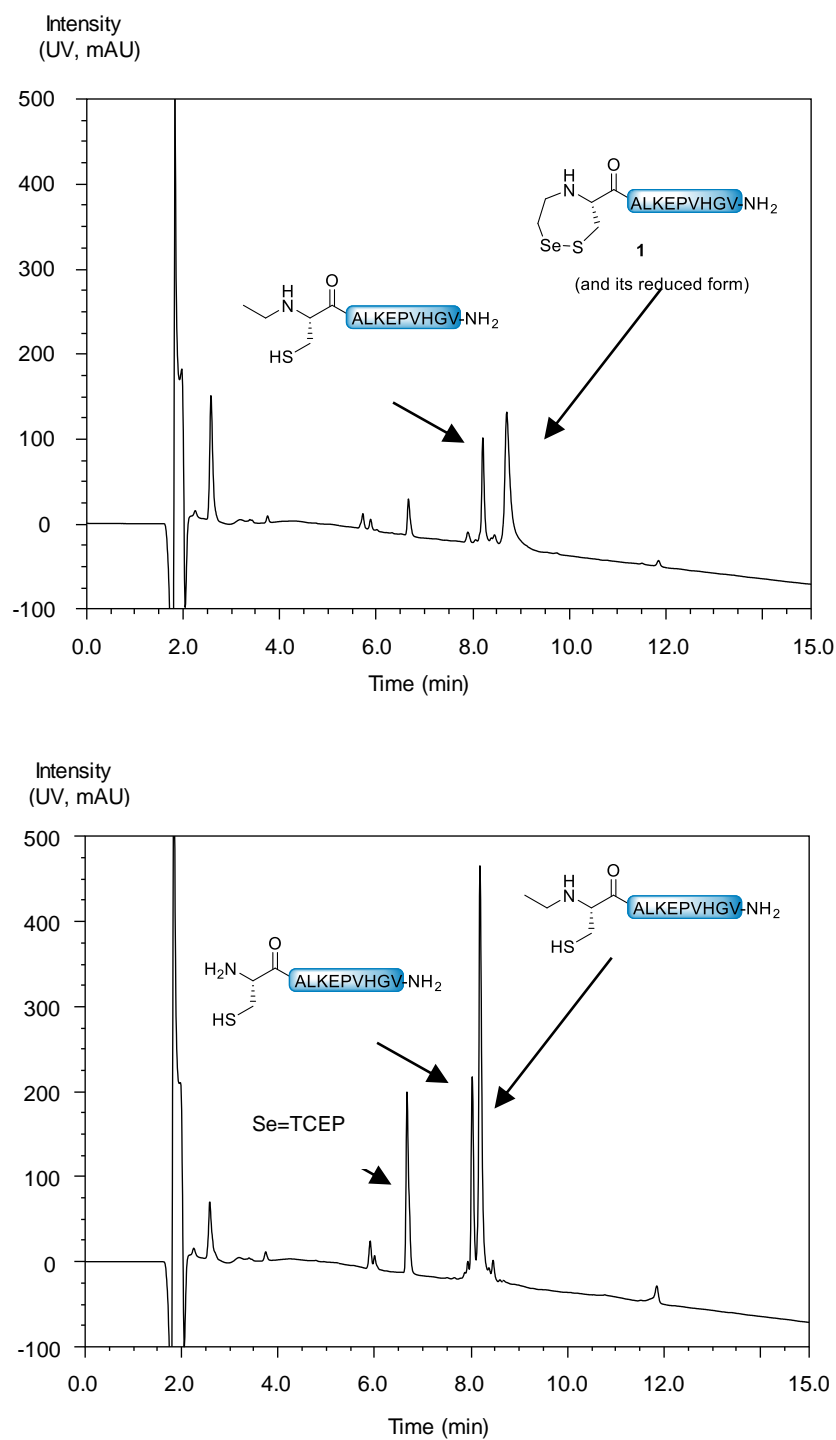

Figure S 53. HPLC monitoring of the treatment of SetCys peptide 1 by TCEP in the absence of ascorbate performed at $\mathrm{pH}$ 6.0. Eluent A: $0.10 \%$ TFA in water, eluent B: $0.10 \%$ TFA in $\mathrm{CH}_{3} \mathrm{CN}$, gradient: $0-50 \% \mathrm{~B}$ in $15 \mathrm{~min}, 30{ }^{\circ} \mathrm{C}, 1 \mathrm{~mL} / \mathrm{min}, \mathrm{C} 18$ column, UV detection $(215 \mathrm{~nm})$. 

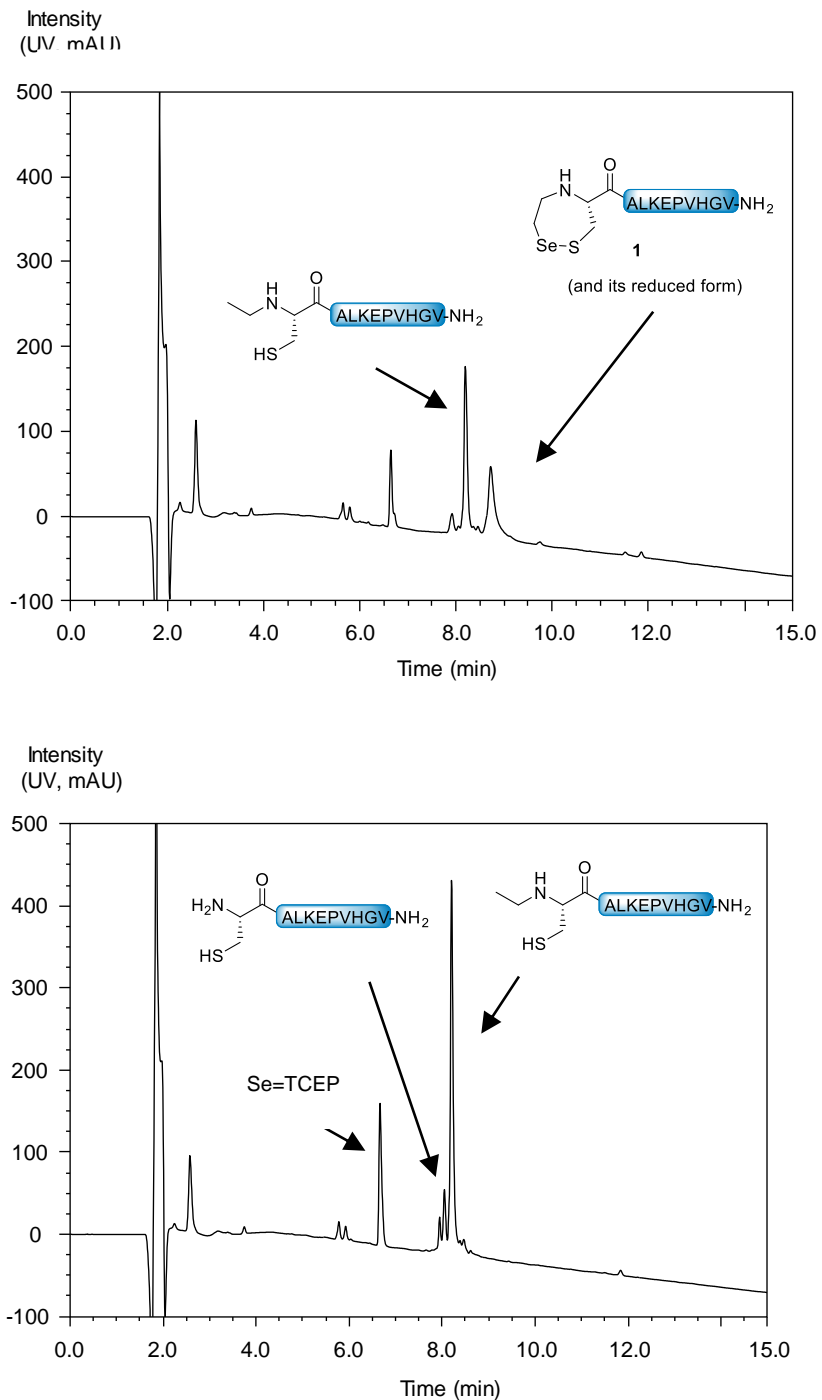

Figure S 54. HPLC monitoring of the treatment of SetCys peptide 1 by TCEP in the absence of ascorbate performed at $\mathrm{pH} 7.20$. Eluent A: $0.10 \%$ TFA in water, eluent $\mathrm{B}: 0.10 \%$ TFA in $\mathrm{CH}_{3} \mathrm{CN}$, gradient: $0-50 \% \mathrm{~B}$ in $15 \mathrm{~min}, 30{ }^{\circ} \mathrm{C}, 1 \mathrm{~mL} / \mathrm{min}, \mathrm{C} 18$ column, UV detection $(215 \mathrm{~nm})$.

The Et-CALKEPVHGV-NH2 peptide produced by deselenization of SetCys peptide $\mathbf{1}$ under the above conditions was isolated by HPLC (Figure S 55) and its sequence was confirmed by MALDI MS-MS analysis (Figure S 56, Figure S 57). 

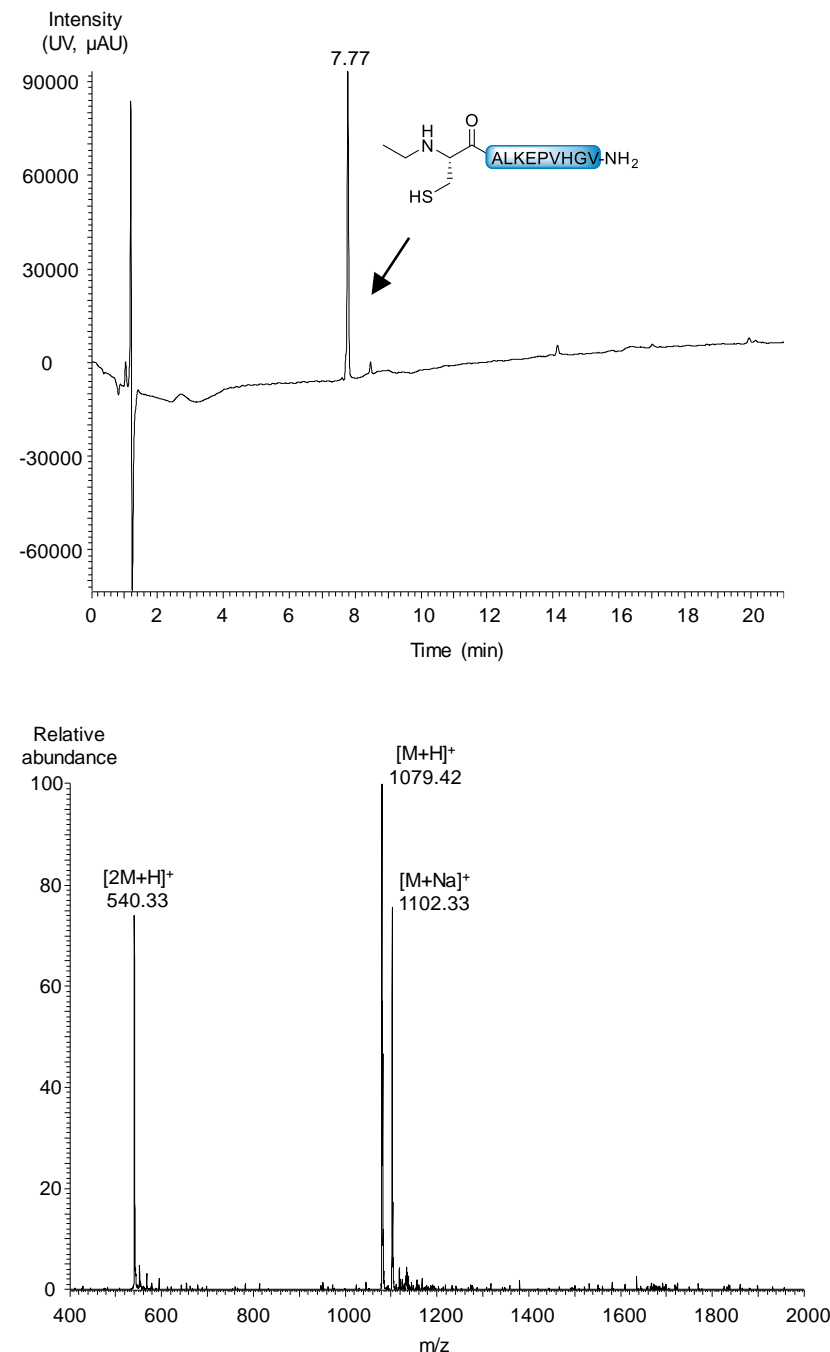

Figure S 55. UPLC-MS analysis of Et-CALKEPVHGV-NH2. a) LC trace. Eluent A: $0.10 \%$ TFA in water, eluent B: $0.10 \%$ TFA in $\mathrm{CH}_{3} \mathrm{CN}$, ACQUITY UPLC Peptide BEH $300 \AA 1.7 \mu \mathrm{m}$ $\left(2.1 \times 150 \mathrm{~mm}\right.$ ) column, $50{ }^{\circ} \mathrm{C}$, gradient $0-70 \% \mathrm{~B}$ in $20 \mathrm{~min}, 0.4 \mathrm{~mL} / \mathrm{min}$, UV detection $(215$ $\mathrm{nm})$. b) MS trace. Calcd. for $[\mathrm{M}+\mathrm{H}]^{+}$(monoisotopic): 1079.60, found: 1079.42 . 


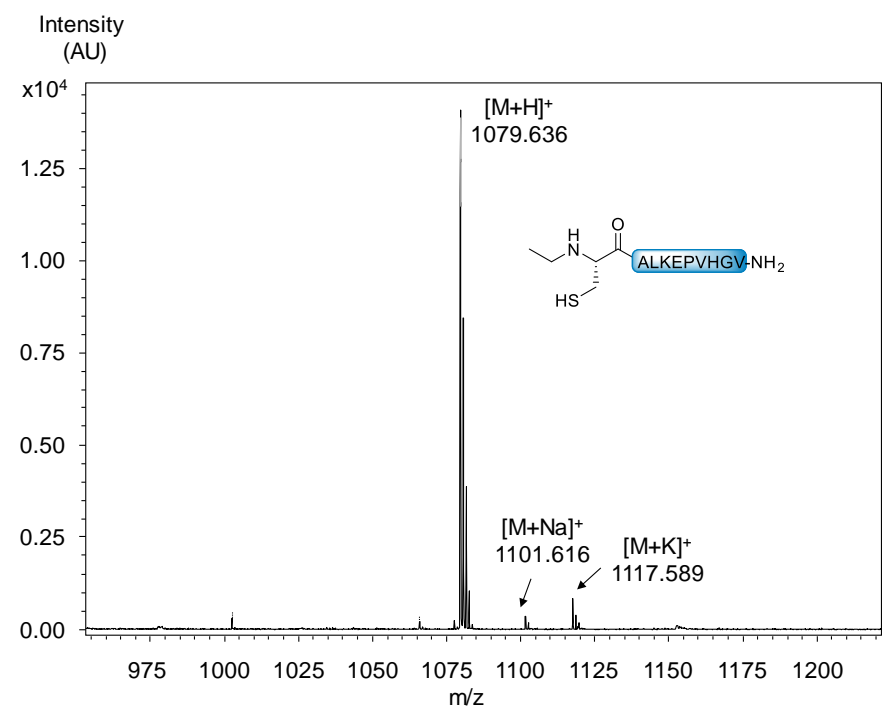

Figure S 56. MALDI-TOF analysis of Et-CALKEPVHGV-NH2 peptide. Matrix $\alpha$-cyano-4hydroxycinnamic acid, positive detection mode, calcd. for $[\mathrm{M}+\mathrm{H}]^{+}$(monoisotopic): 1079.60, found: 1079.64 .

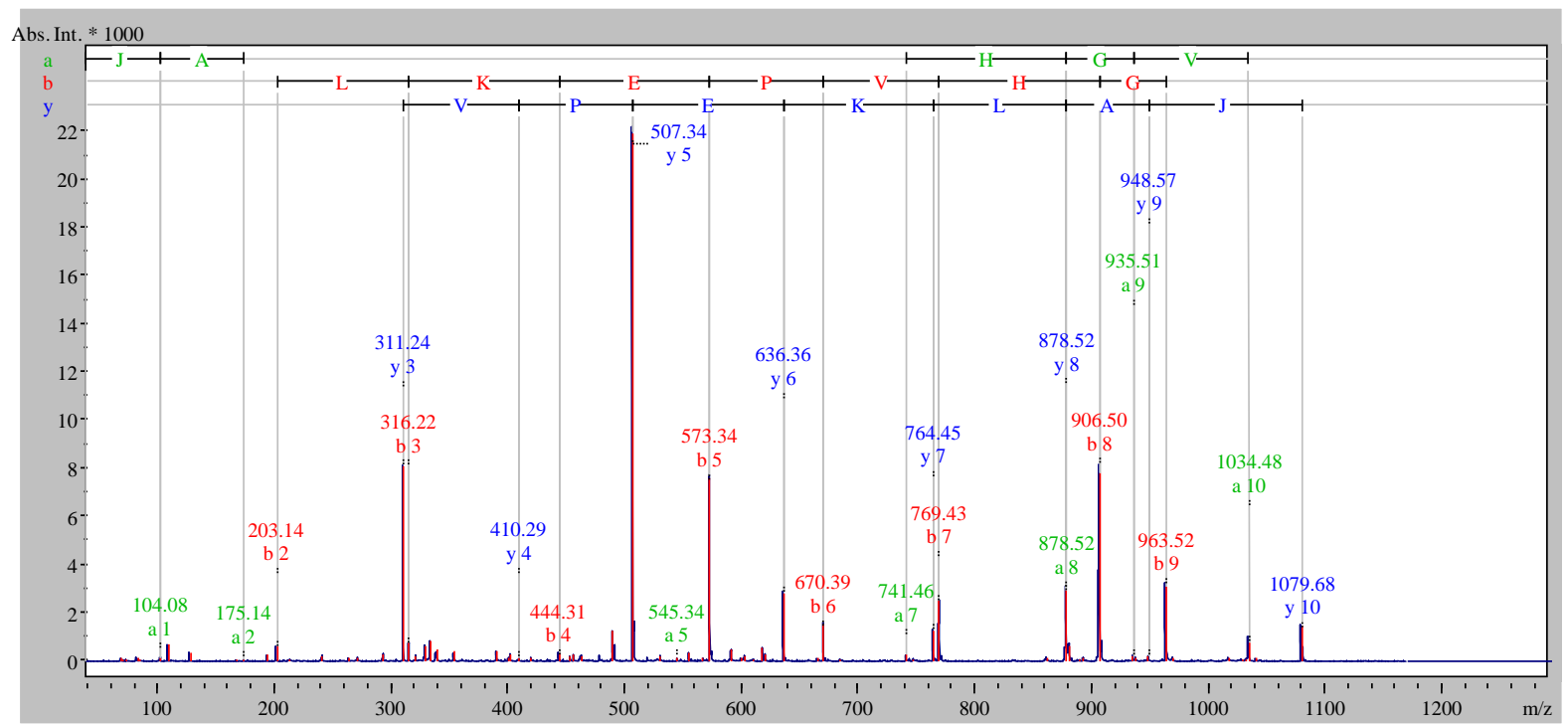

Figure S 57. MALDI-TOF-TOF sequencing of Et-CALKEPVHGV-NH2 peptide. Matrix $\alpha$ cyano-4-hydroxycinnamic acid, positive detection mode. The MS-MS analysis confirms that the SetCys residue is converted into a $N$-ethyl cysteine residue ( $\mathrm{J}$ in the figure) in the peptide sequence. 


\subsection{NCL under low reducing conditions (properties 2)}

When SetCys peptide $\mathbf{1}$ and peptide thioester $\mathbf{4}$ were reacted under mild reducing conditions (MPAA), the resulting peptide featuring a SetCys unit in the form of a cyclic selenosulfide at the ligation junction was recovered as the only product of the ligation with $41 \%$ yield (Figure S 58). The insertion of the SetCys amino acid into the peptide sequence was confirmed by MSMS analysis (Figure S 62).

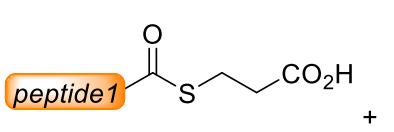

4 (8 mM, 1 eq)

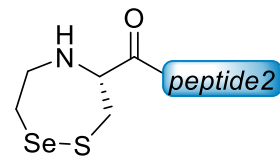

$1 \quad(1.2 \mathrm{eq})$

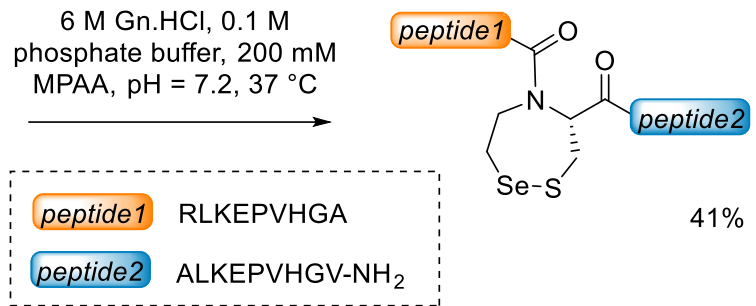

Figure S 58. Ligation reaction of SetCys peptide 1 with peptidyl MPA-thioester 4 .

\section{Protocol}

To a solution of $\mathrm{Gn} \cdot \mathrm{HCl}(1.03 \mathrm{~g})$ in $0.1 \mathrm{M}, \mathrm{pH} 7.4$ phosphate buffer $(1.08 \mathrm{~mL})$ was added MPAA $(60.6 \mathrm{mg})$ and the $\mathrm{pH}$ of the mixture was adjusted to 7.20 by addition of $6 \mathrm{M} \mathrm{NaOH}$. SetCys-ALKEPVHGV-NH $\mathbf{1}(23.2 \mathrm{mg}, 15.5 \mu \mathrm{mol}, 1.2 \mathrm{eq})$ and RLKEPVHGA-MPA 4 (20.0 $\mathrm{mg}, 12.9 \mu \mathrm{mol}, 1 \mathrm{eq})$ were then successively dissolved in this solution $(1.61 \mathrm{~mL})$ and the reaction mixture was stirred at $37^{\circ} \mathrm{C}$ for $24 \mathrm{~h}$. The mixture was then acidified with $10 \% \mathrm{AcOH}$ in water $(22.5 \mathrm{~mL})$ and extracted with $\mathrm{Et}_{2} \mathrm{O}(5 \times)$ to remove the MPAA. Purification of the crude by HPLC (eluent $\mathrm{A}=$ water containing $0.1 \%$ TFA, eluent $\mathrm{B}=$ acetonitrile in water $4 / 1$ containing $0.1 \% \mathrm{TFA}, 50^{\circ} \mathrm{C}$, detection at $215 \mathrm{~nm}, 3 \mathrm{~mL} / \mathrm{min}, 0-10 \%$ eluent $\mathrm{B}$ in $5 \mathrm{~min}$, then 10 $35 \%$ eluent B in $35 \mathrm{~min}$, then $35-50 \%$ eluent B in $15 \mathrm{~min}, \mathrm{C} 18 \mathrm{XBridge}$ column) provided RLKEPVHGA-SetCys-ALKEPVHGV-NH $\mathbf{N}_{2}$ as a white solid after lyophilisation (15.06 $\mathrm{mg}$, $41 \%)$. 


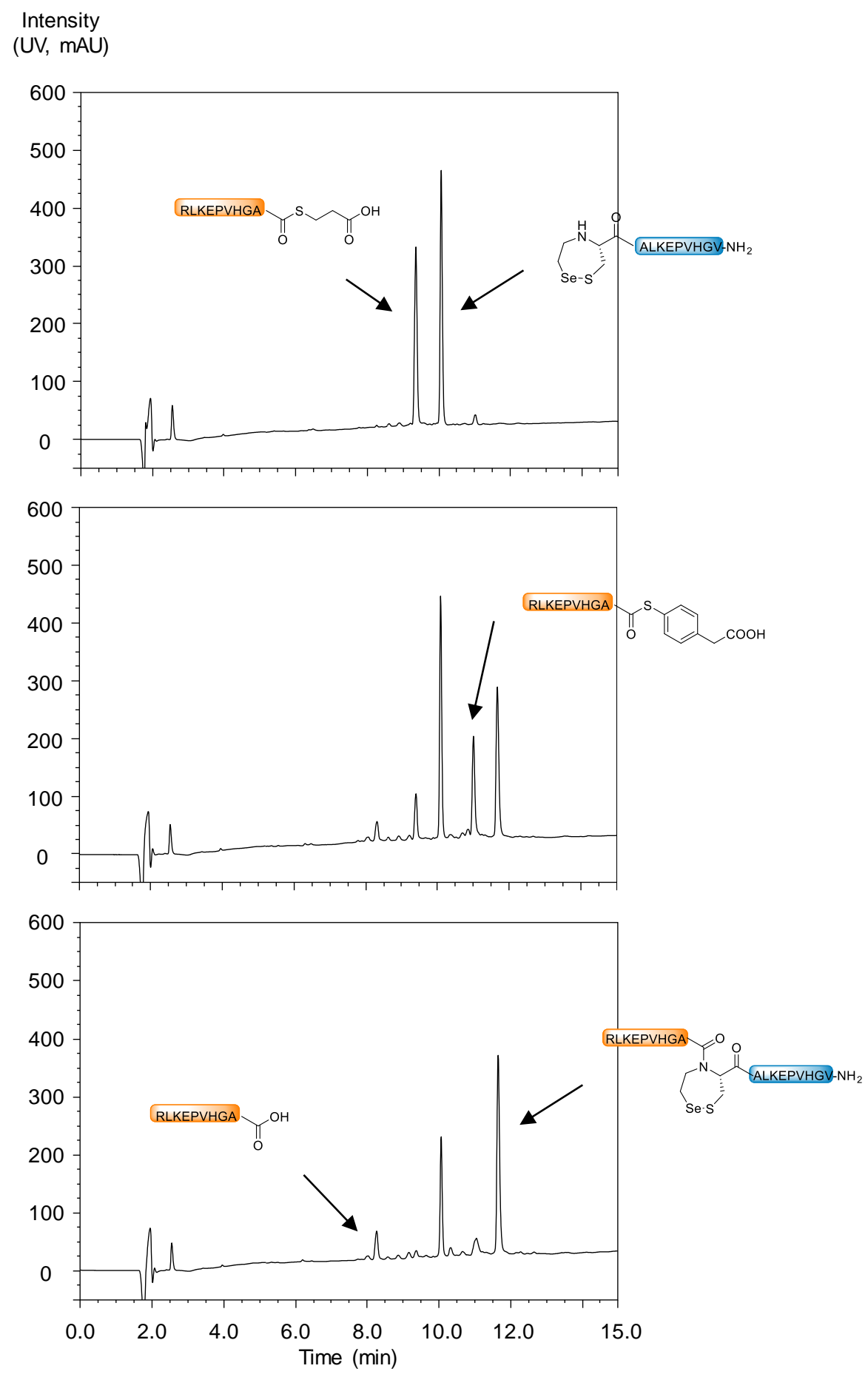

Figure S 59. Monitoring of the ligation between SetCys peptide 1 and peptidyl MPA thioester RLKEPVHGA-MPA. LC trace: eluent A $0.10 \%$ TFA in water, eluent B $0.10 \%$ TFA in $\mathrm{CH}_{3} \mathrm{CN} /$ water: $4 / 1$ by vol., $\mathrm{C} 18$ column, gradient $0-50 \% \mathrm{~B}$ in $15 \mathrm{~min}, 30{ }^{\circ} \mathrm{C}, 1 \mathrm{~mL} / \mathrm{min}, \mathrm{UV}$ detection at $215 \mathrm{~nm}$. 


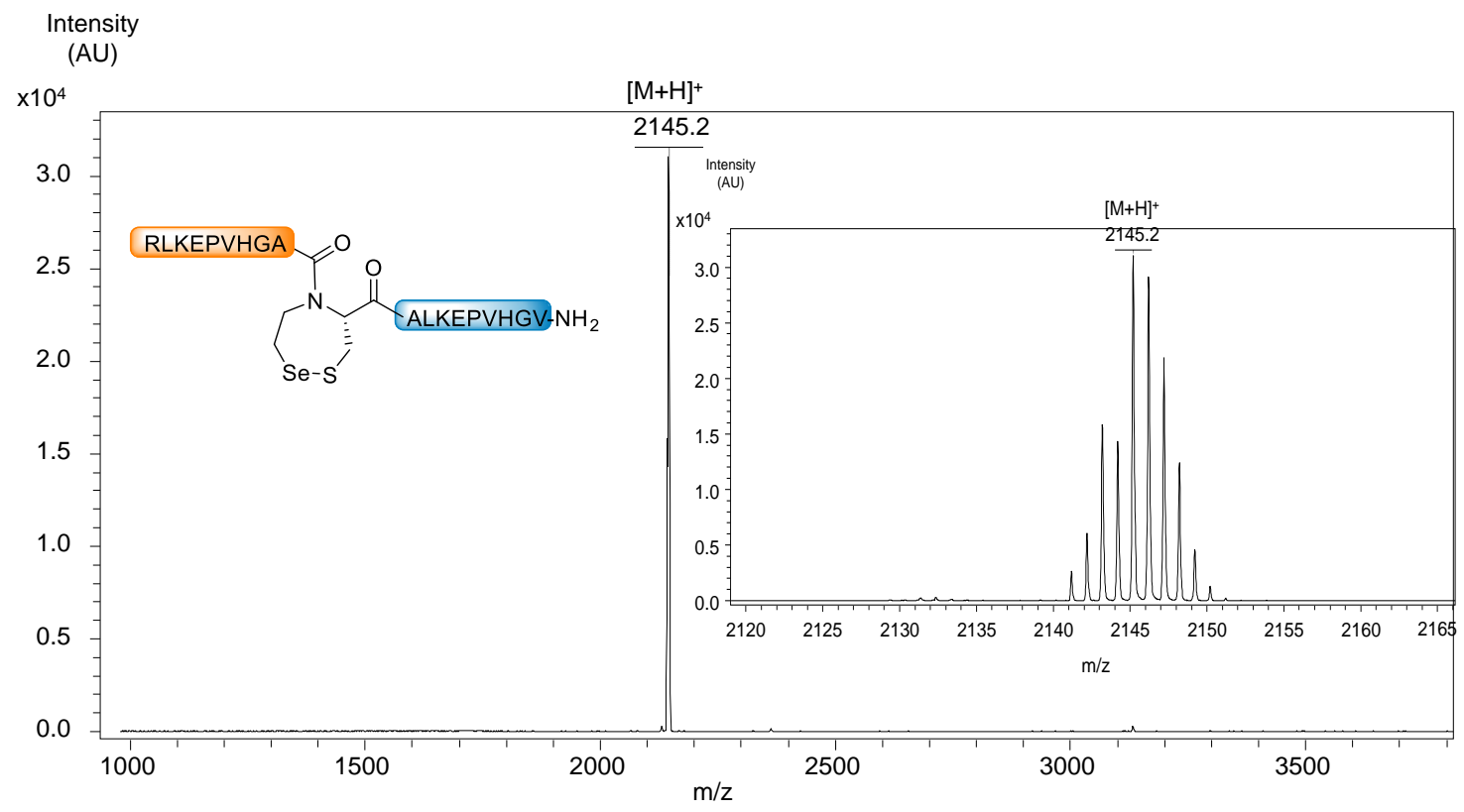

Figure S 60. MALDI-TOF analysis of RLKEPVHGA-SetCys-ALKEPVHGV-NH2 peptide. Matrix $\alpha$-cyano-4-hydroxycinnamic acid, positive detection mode, calcd. for $[\mathrm{M}+\mathrm{H}]^{+}$ (monoisotopic): 2145.06, found: 2145.2.

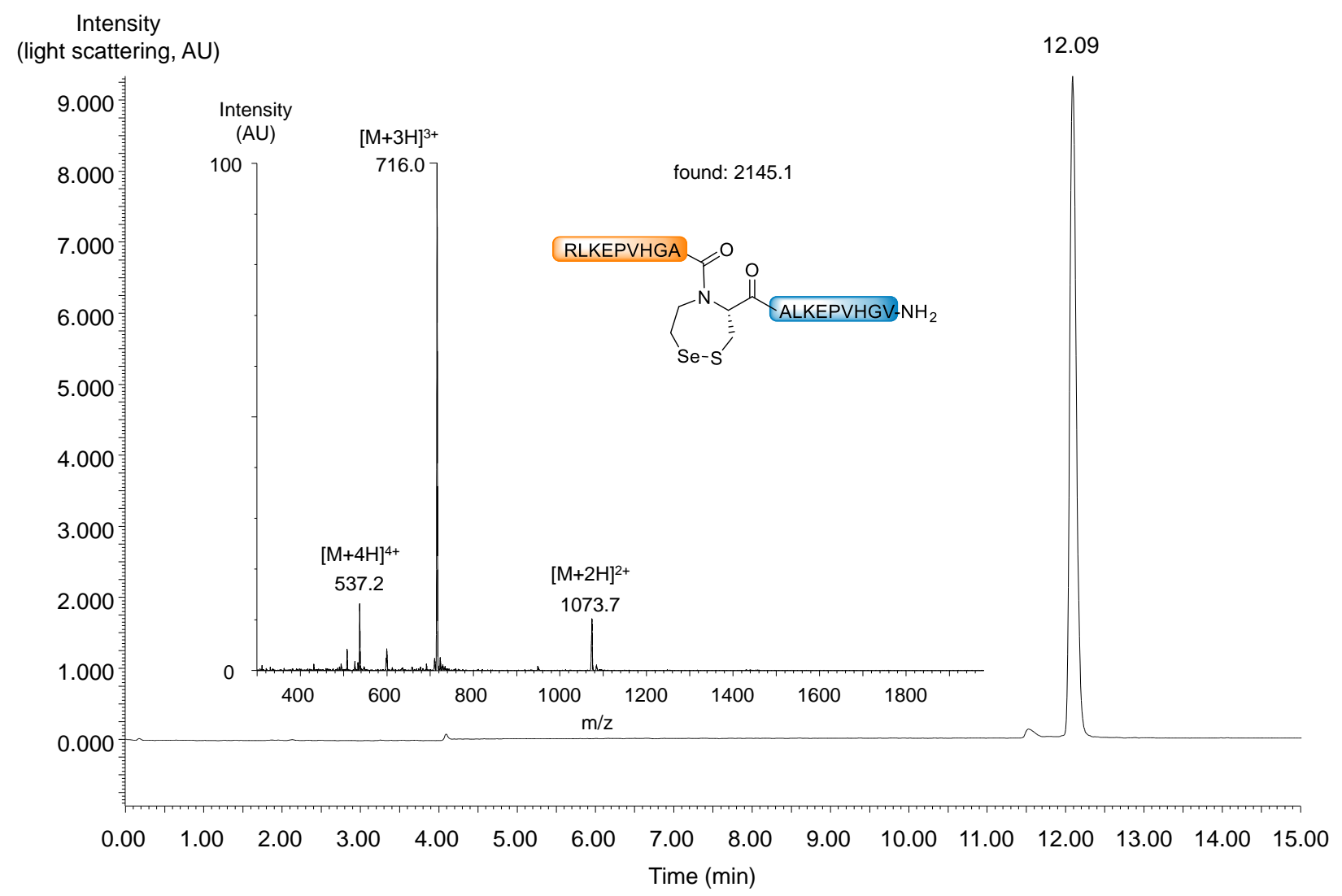

Figure S 61. LC-MS analysis of RLKEPVHGA-SetCys-ALKEPVHGV-NH2 peptide. LC trace: eluent A $0.10 \%$ TFA in water, eluent B $0.10 \%$ TFA in $\mathrm{CH}_{3} \mathrm{CN} /$ water: $4 / 1$ by vol. $\mathrm{C} 18$ Xbridge BEH $300 \AA 5 \mu \mathrm{m}(4.6 \times 250 \mathrm{~mm})$ column, gradient $0-50 \% \mathrm{~B}$ in $15 \mathrm{~min}, 30^{\circ} \mathrm{C}, 1$ 
$\mathrm{mL} / \mathrm{min}$, light scattering detection. MS trace: $1073.7\left([\mathrm{M}+2 \mathrm{H}]^{2+}\right), 716.0\left([\mathrm{M}+3 \mathrm{H}]^{3+}\right), 537.2$ $\left([\mathrm{M}+4 \mathrm{H}]^{4+}\right)$; calcd. for M (average): 2144.4, found: 2145.1 .

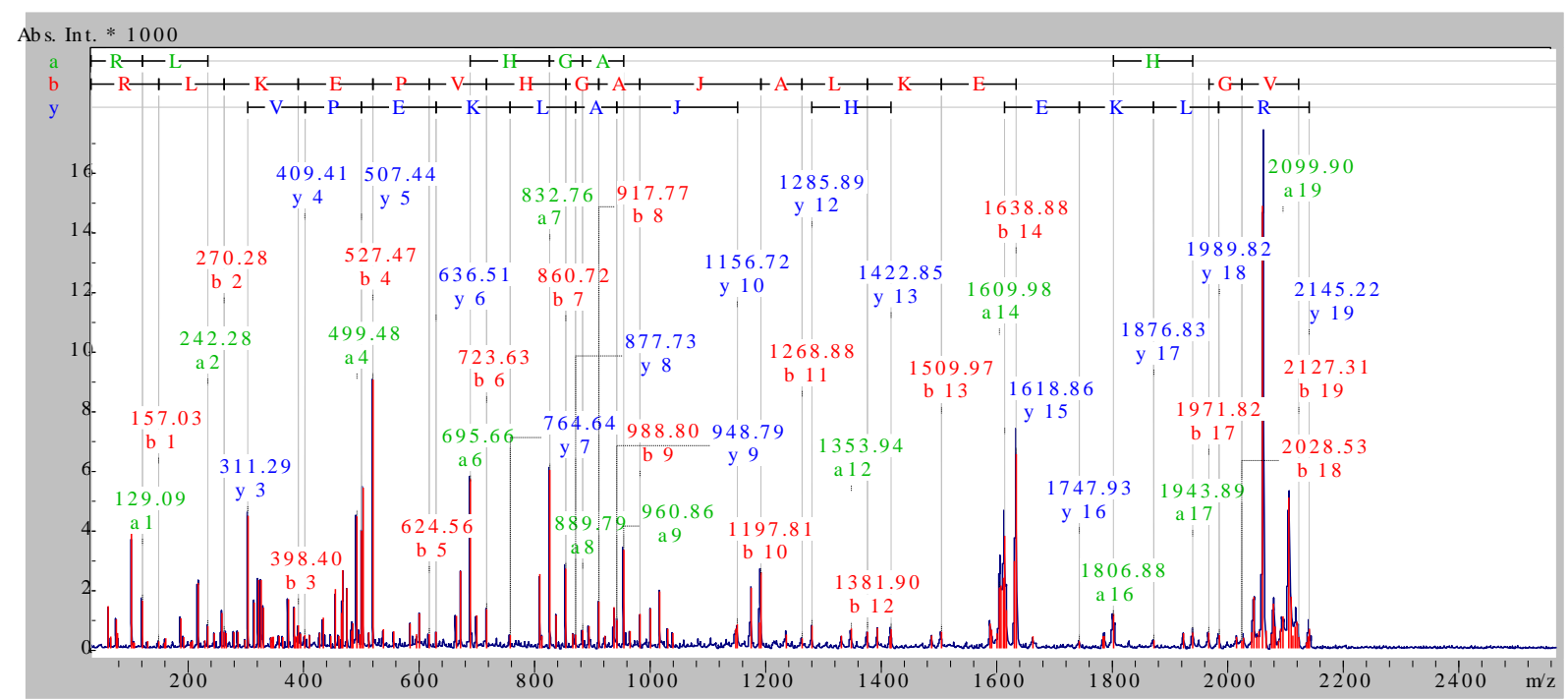

Figure S 62. MALDI-TOF-TOF sequencing of RLKEPVHGA-SetCys-ALKEPVHGV-NH2 peptide. Matrix $\alpha$-cyano-4-hydroxycinnamic acid, positive detection mode. The MS-MS analysis confirms the presence of SetCys ( $\mathrm{J}$ on the figure) residue in the sequence of the peptide.

\section{Kinetic study}

The progress of the reaction was monitored by HPLC. For each point, a $2 \mu \mathrm{L}$ aliquot was taken from the reaction mixture and quenched by adding $10 \% \mathrm{AcOH}$ in water $(100 \mu \mathrm{L})$. The sample was then extracted with $\mathrm{Et}_{2} \mathrm{O}$ to remove MPAA prior to HPLC analysis. The monitoring of the ligation points out that the MPAA peptide thioester produced from RLKEPVHGA-MPA 4 by transthioesterification with MPAA quickly accumulates in the reaction mixture. This intermediate is then slowly consumed with concomitant formation of the ligated product. 


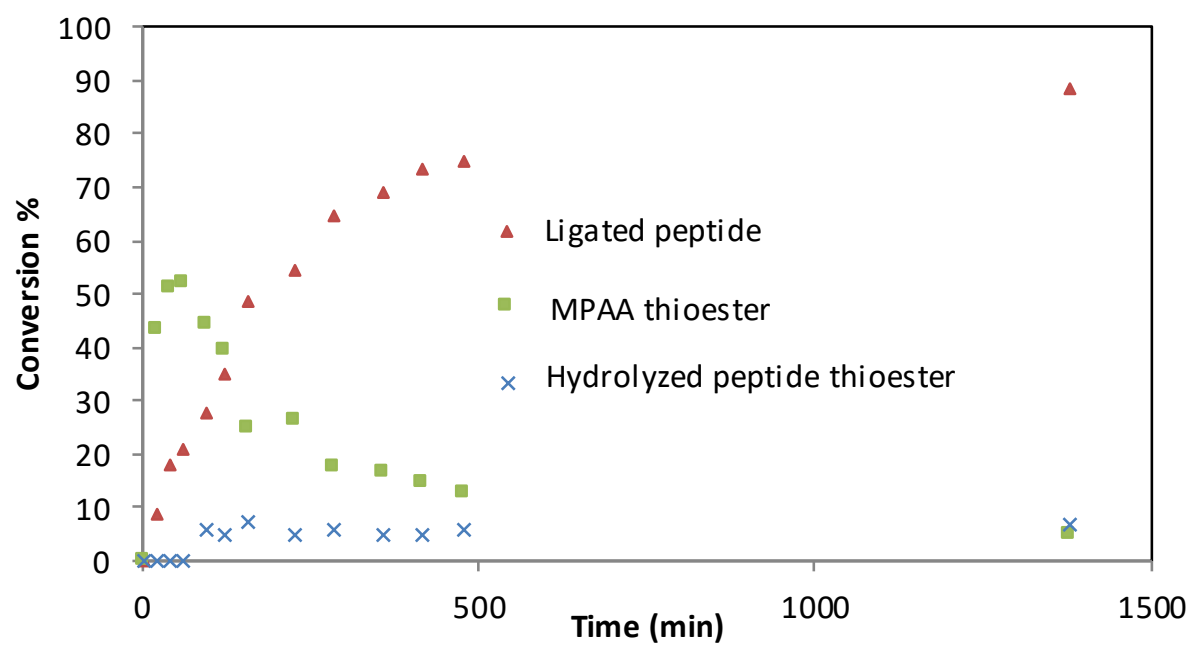

Figure S 63. Ligation between SetCys peptide 1 and peptide thioester 4 performed under low reductive conditions (MPAA): evolution of the reaction mixture composition. The proportion of each species in the mixture was determined by HPLC using UV detection at $215 \mathrm{~nm}$.

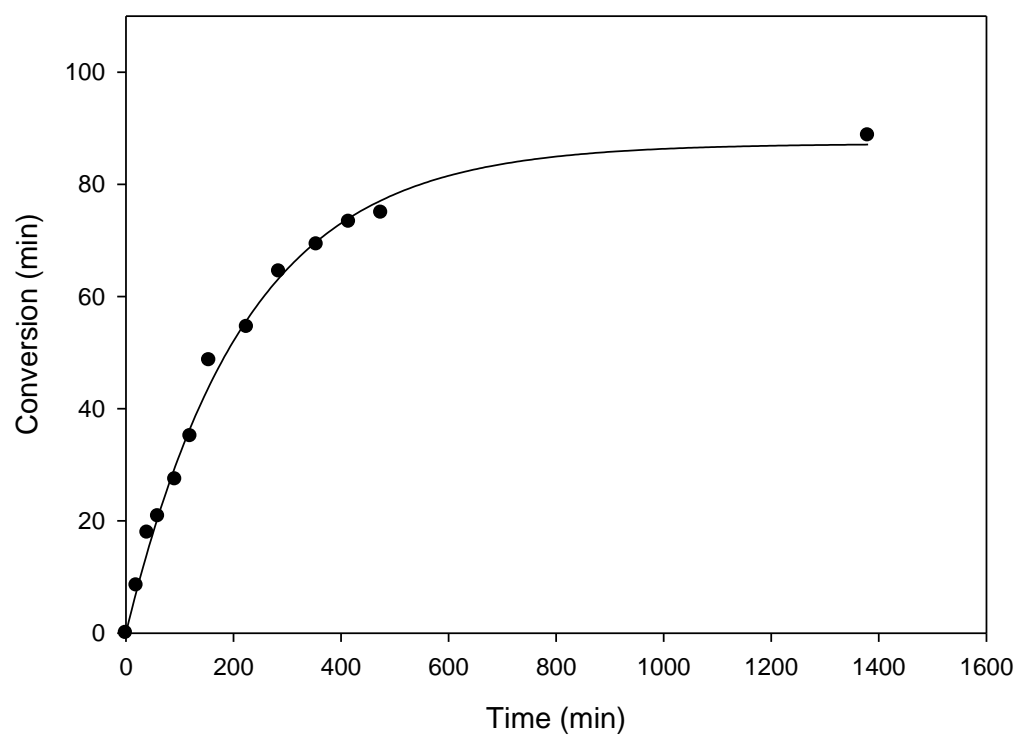

Figure S 64. Fitting of the reaction between SetCys peptide 1 and peptide thioester 4 using SigmaPlot Software $\left(\mathrm{k}_{1}=0.0045 \mathrm{~min}^{-1} ; \mathrm{t}_{1 / 2}=2.57 \mathrm{~h}\right)$. Black dots correspond to experimental values. 


\subsection{SetCys is silent during NCL under low reducing conditions (property 3)}

\subsubsection{Competitive ligation experiment involving a Cys and SetCys peptide}

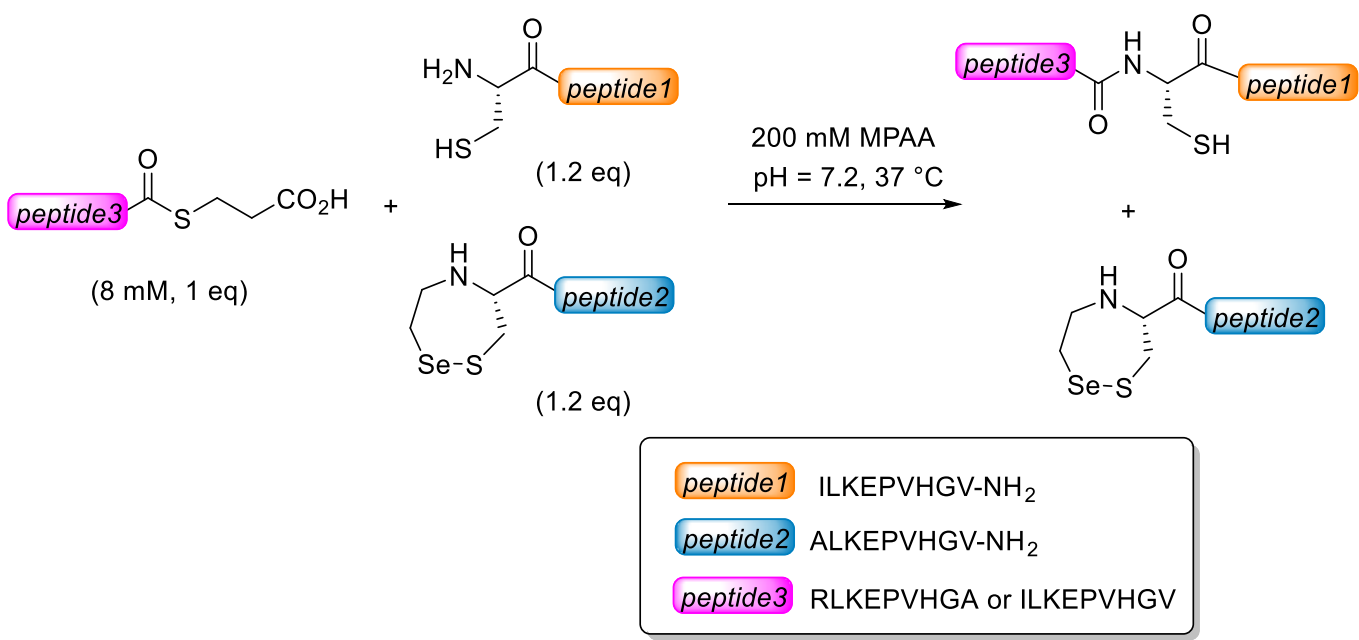

Figure S 65. SetCys is silent during NCL under low reducing conditions.

General procedure illustrated with RLKEPVHGA-MPA 4 as peptide thioester:

To a solution of $\mathrm{Gn} \cdot \mathrm{HCl}(287 \mathrm{mg})$ in $0.1 \mathrm{M}$, pH 7.4 phosphate buffer $(300 \mu \mathrm{L})$ was added MPAA $(16.8 \mathrm{mg}$ ) and the $\mathrm{pH}$ of the mixture was adjusted to 7.22 by addition of $6 \mathrm{M} \mathrm{NaOH}$. SetCys-ALKEPVHGV-NH2 (1.32 mg, $0.88 \mu \mathrm{mol}, 1.2 \mathrm{eq})$, CILKEPVHGV-NH2 $(1.27 \mathrm{mg}$, $0.88 \mu \mathrm{mol}, 1.2 \mathrm{eq})$ and RLKEPVHGA-MPA $(1.14 \mathrm{mg}, 0.735 \mu \mathrm{mol}, 1 \mathrm{eq})$ were then successively dissolved in this solution $(92 \mu \mathrm{L})$ and the reaction mixture was stirred at $37^{\circ} \mathrm{C}$.

The progress of the kinetically favored ligation (RLKEPVHGA-MPA + CILKEPVHGVNH2) was monitored by HPLC. For each point, a $2 \mu \mathrm{L}$ aliquot was taken from the reaction mixture and quenched by adding $10 \% \mathrm{AcOH}$ in water $(100 \mu \mathrm{L})$. The sample was then extracted with $\mathrm{Et}_{2} \mathrm{O}$ to remove MPAA prior to HPLC analysis. The kinetic data were then fitted to a pseudo-first order kinetic law and the results are given in Table $S 2$.

The reaction was repeated using ILKEPVHGV-MPA instead RLKEPVHGA-MPA as peptidyl thioester. In both cases, SetCys-ALKEPVHGV-NH2 peptide was recovered unreacted at the end of the ligation (Figure S 67, Figure S 68). 


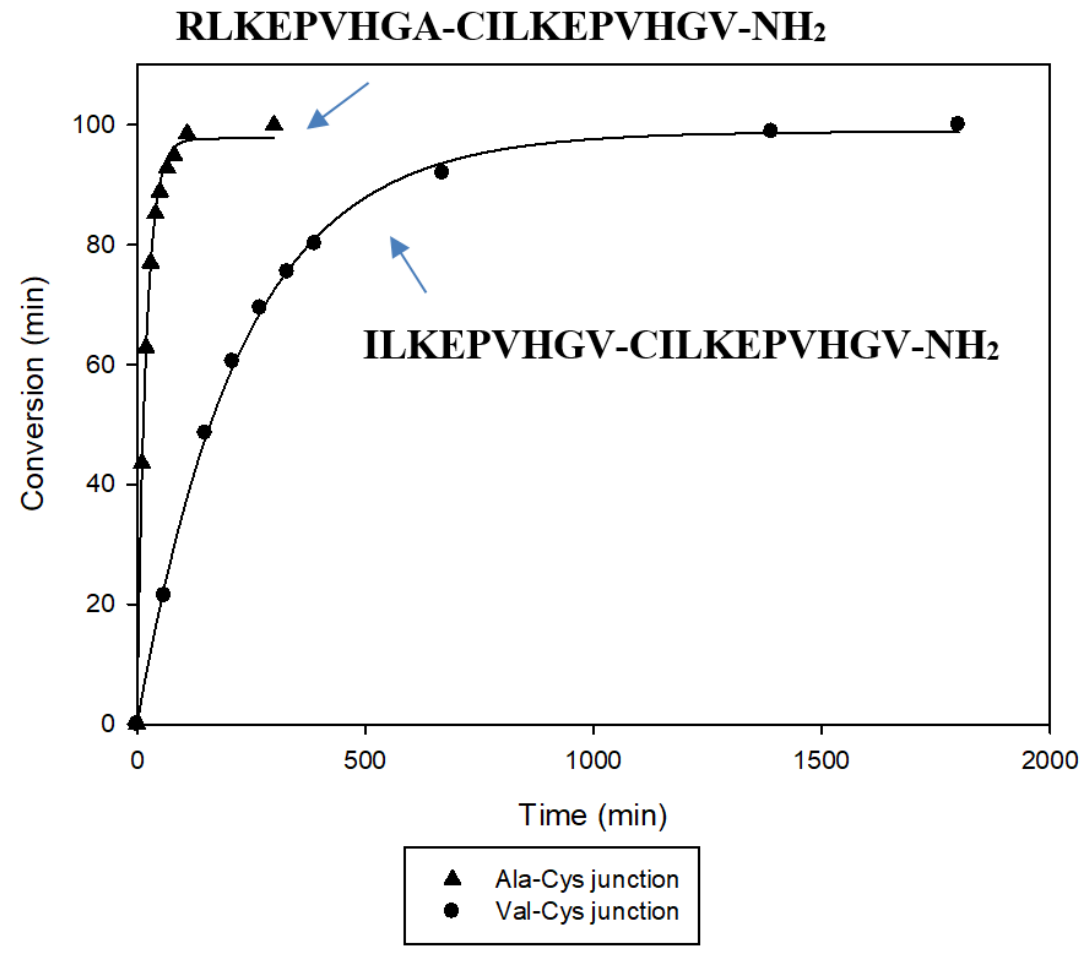

Figure S 66. Ligations using respectively RLKEPVHGA-MPA and ILKEPVHGV-MPA as acyl donor. The continuous curves correspond to the fitting to a pseudo first order kinetic law from which $\mathrm{t}^{1 / 2}$ and $\mathrm{k}_{1}$ were extracted.

Table S 2. Rate constants and half-reaction times determined from data in Figure $\mathbf{S} 66$.

\begin{tabular}{ccc} 
Product of ligation & $\mathbf{k 1}\left(\mathbf{m i n}^{-\mathbf{1}}\right)$ & $\mathbf{t} \mathbf{1} / \mathbf{2}(\mathbf{h})$ \\
\hline RLKEPVHGA-CILKEPVHGV-NH & 0.0512 & 0.22 \\
ILKEPVHGV-CILKEPVHGV-NH & 0.0044 & 2.62
\end{tabular}



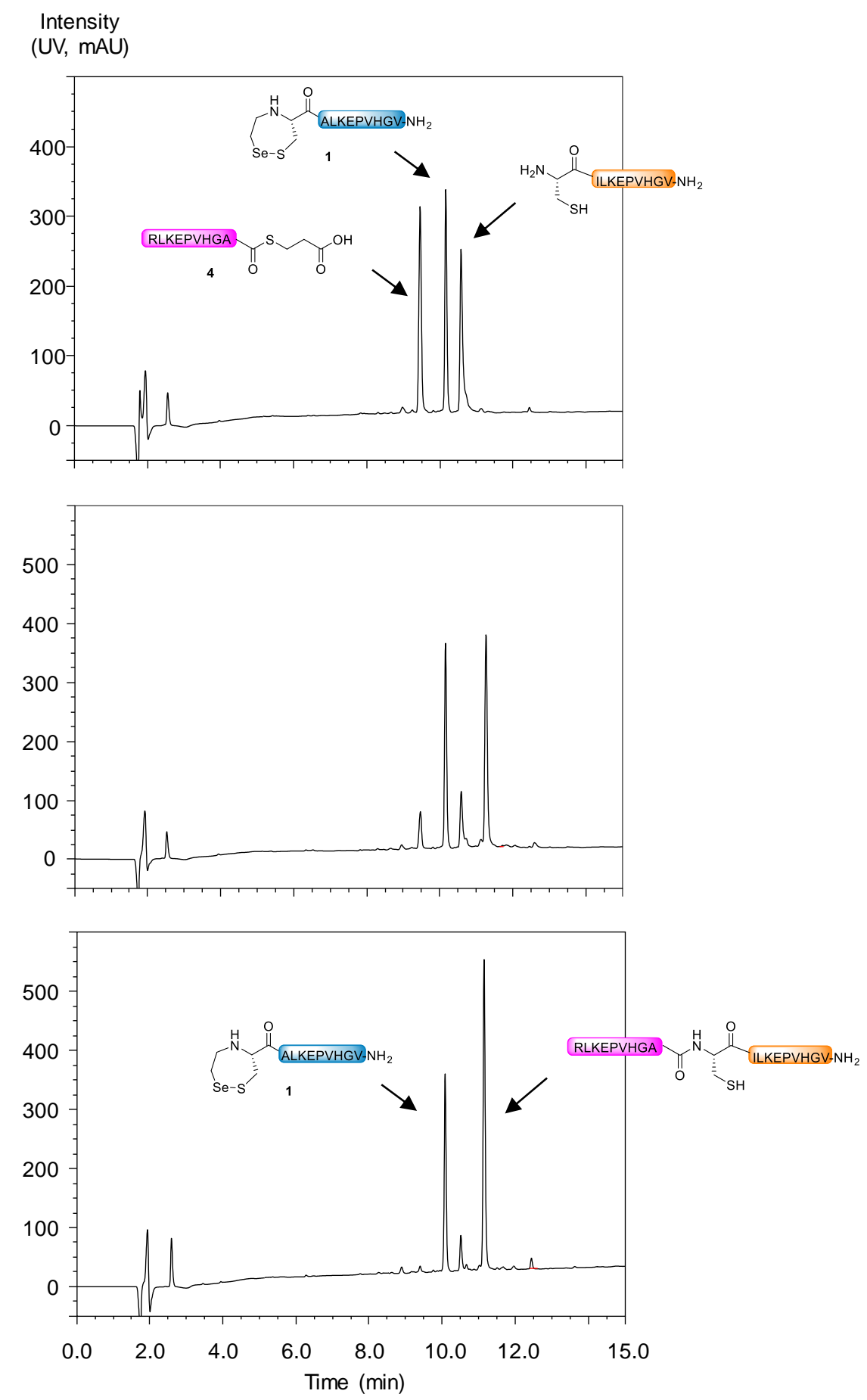

Figure S 67. Monitoring of the competitive reaction between SetCys peptide 1 and cysteinyl peptide CILKEPVHGV-NH 2 with peptidyl MPA-thioester 4. LC trace: eluent A $0.10 \%$ TFA in water, eluent $\mathrm{B} 0.10 \%$ TFA in $\mathrm{CH}_{3} \mathrm{CN} /$ water: $4 / 1$ by vol., $\mathrm{C} 18$ column, gradient $0-50 \%$ B in 15 $\min , 30{ }^{\circ} \mathrm{C}, 1 \mathrm{~mL} / \mathrm{min}$, UV detection at $215 \mathrm{~nm}$. 


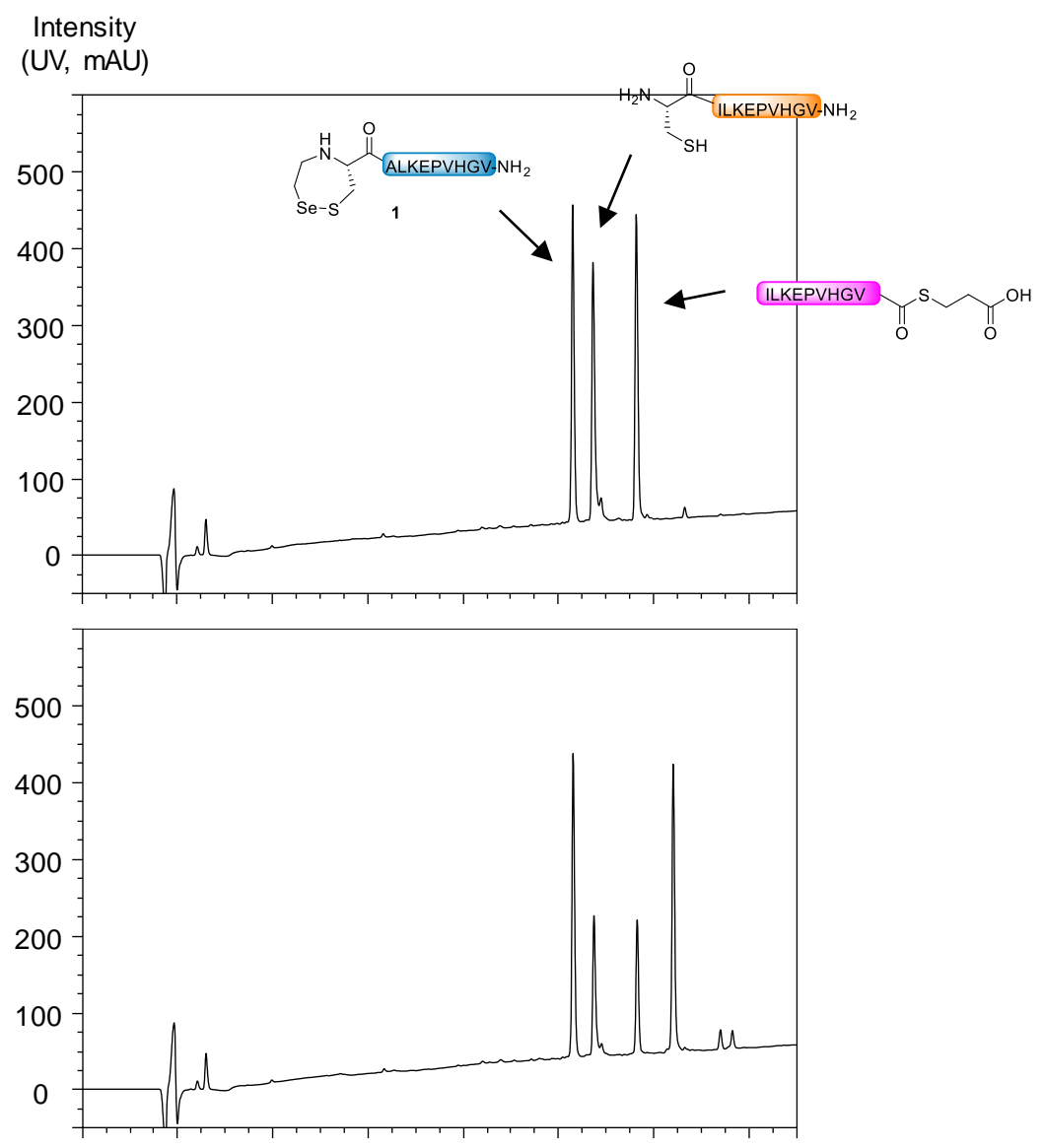

b) $t=23 h$

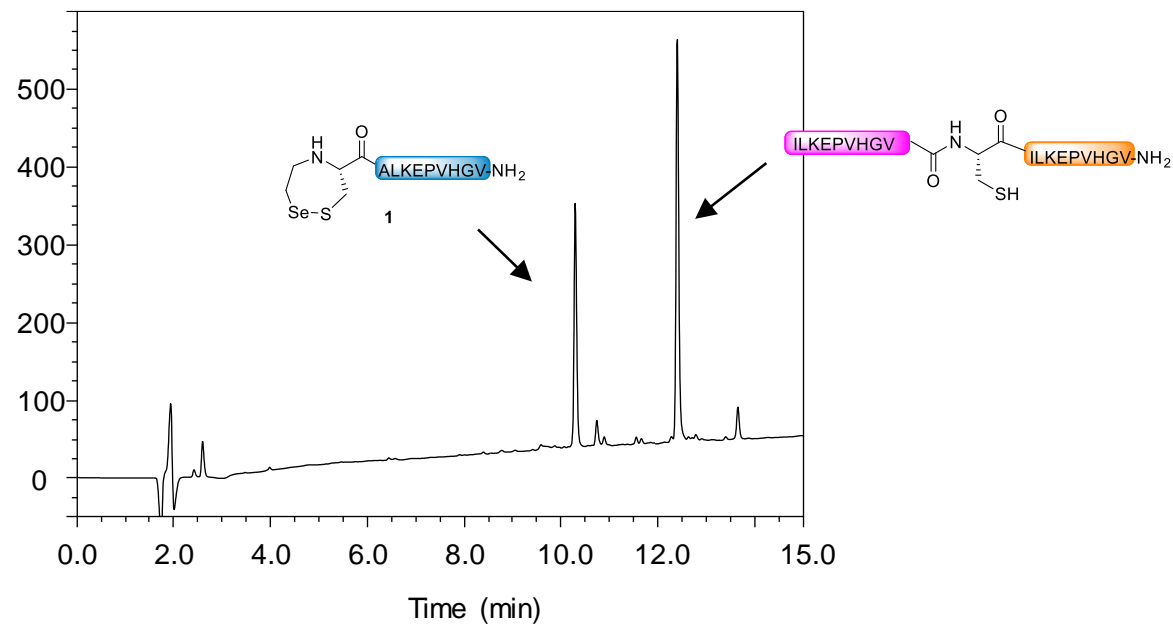

Figure S 68. Monitoring of the competitive reaction between SetCys peptide $\mathbf{1}$ and cysteinyl peptide CILKEPVHGV-NH ${ }_{2}$ with peptidyl MPA-thioester ILKEPVHGV-MPA. LC trace: eluent $\mathrm{A} 0.10 \%$ TFA in water, eluent $\mathrm{B} 0.10 \%$ TFA in $\mathrm{CH}_{3} \mathrm{CN} /$ water: $4 / 1$ by vol., $\mathrm{C} 18$ column, gradient $0-50 \% \mathrm{~B}$ in $15 \mathrm{~min}, 30{ }^{\circ} \mathrm{C}, 1 \mathrm{~mL} / \mathrm{min}$, UV detection at $215 \mathrm{~nm}$. 


\subsubsection{Compatibility of SetCys with Cys-rich peptides}

The Cys-rich peptide chosen for this study is conotoxin OIVA. The linear peptide corresponding to conotoxin OIVA (CCGVONAACHOCVCKNTC-NH $2, \mathrm{O}=$ hydroxyproline) was synthesized following the method described in Figure $S 69$.
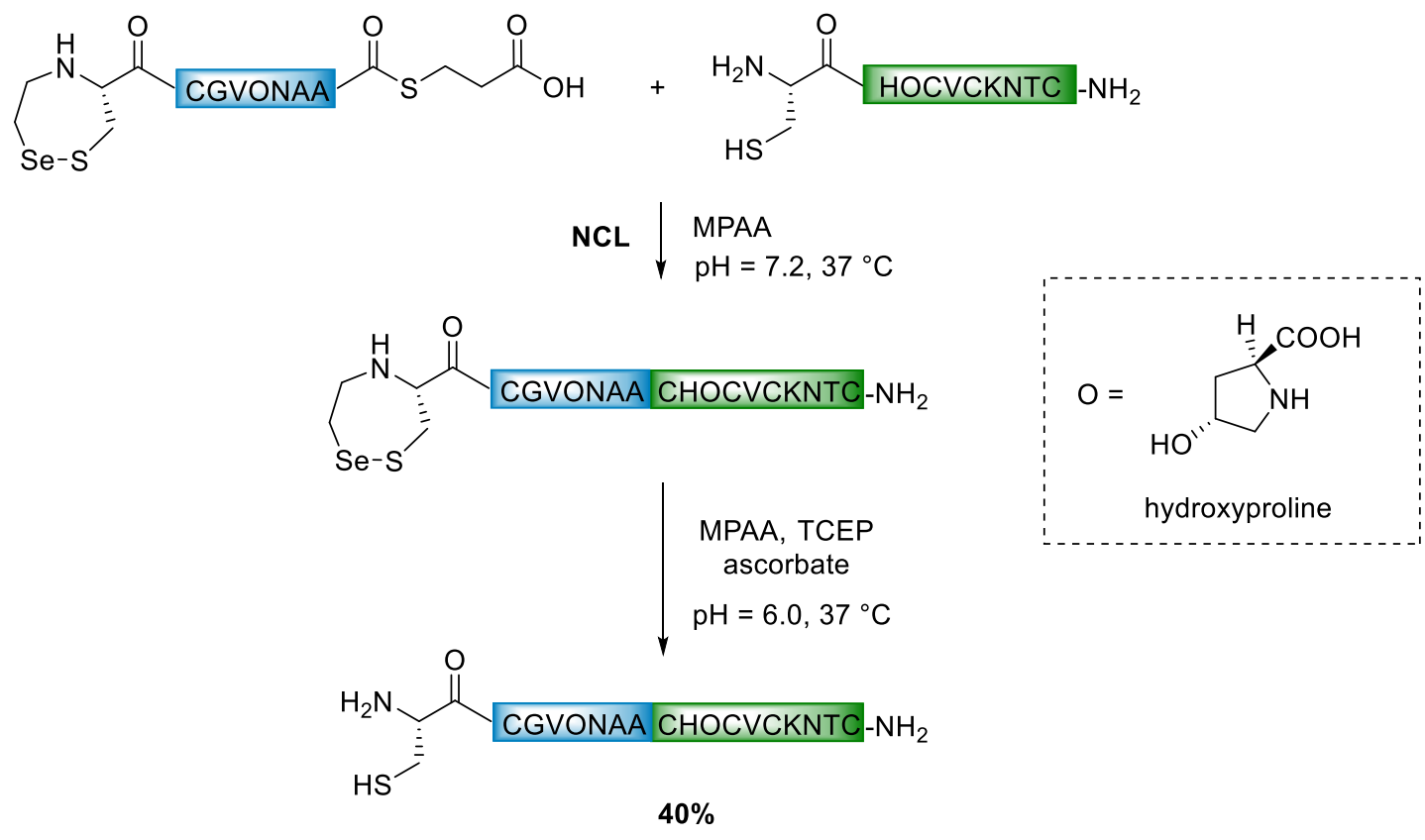

Figure S 69. Synthesis of conotoxin OIVA by ligation of two peptide segments.

\section{Synthesis of CHOCVCKNTC-NH}

CHOCVCKNTC-NH2 peptide was synthesized on $0.1 \mathrm{mmol}$ scale as described in the general procedure and was cleaved from the solid support using TFA/TIS/ $\mathrm{H}_{2} \mathrm{O} /$ thioanisol/thiophenol 92.5:5:2.5:2.5:2.5 $(10 \mathrm{~mL})$. The solid that was recovered by precipitation from $\mathrm{Et}_{2} \mathrm{O} /$ heptane was solubilized in water and lyophilized. Purification of the crude by HPLC (eluent A $0.10 \%$ TFA in water, eluent $\mathrm{B} 0.10 \%$ TFA in $\mathrm{CH}_{3} \mathrm{CN}, 20 \mathrm{~mL} / \mathrm{min}, 0-5 \%$ eluent $\mathrm{B}$ in 10 min then 5$25 \%$ in $25 \mathrm{~min}, \mathrm{C} 18$ column, detection at $215 \mathrm{~nm}$, rt) provided the desired peptide as a white powder after lyophilization $(61.3 \mathrm{mg}, 42 \%)$. 
Characterization of CHOCVCKNTC- $\mathrm{NH}_{2}$

a)

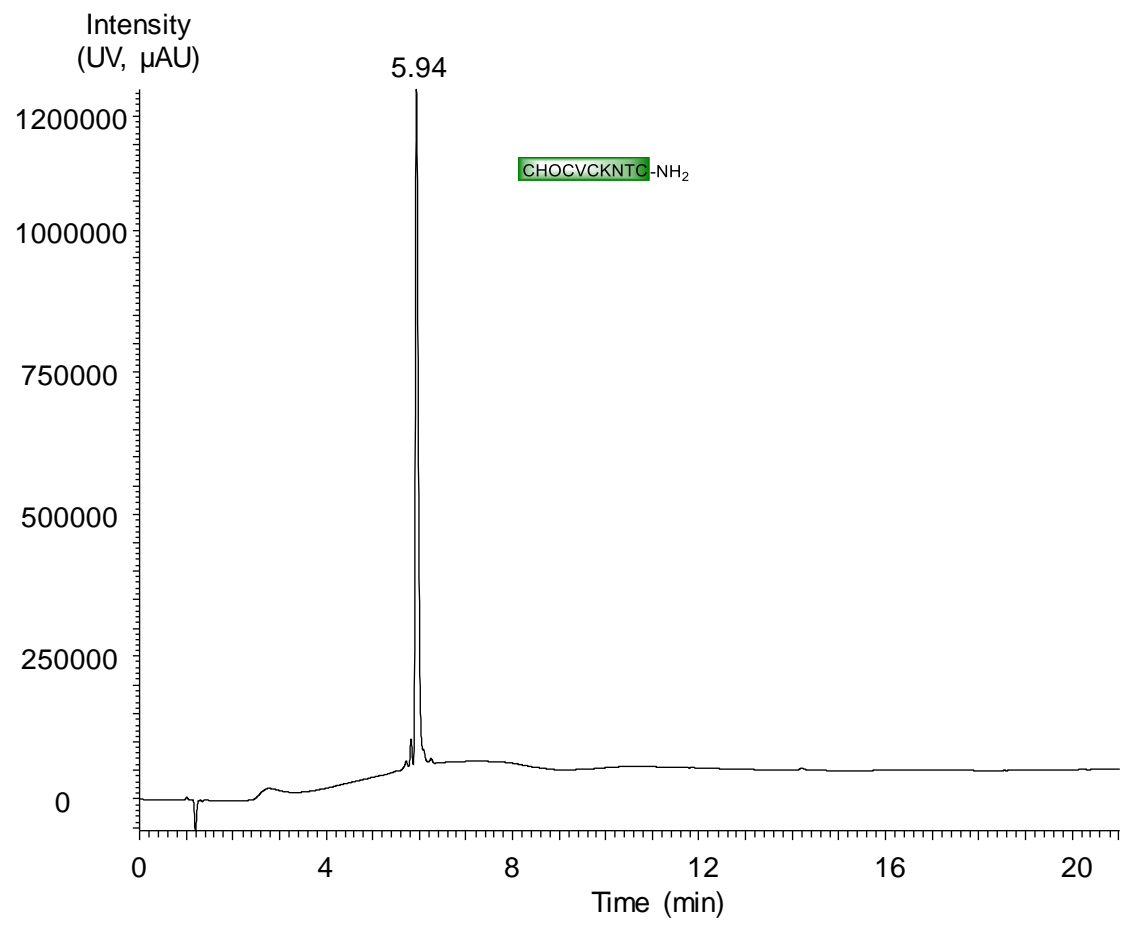

b)

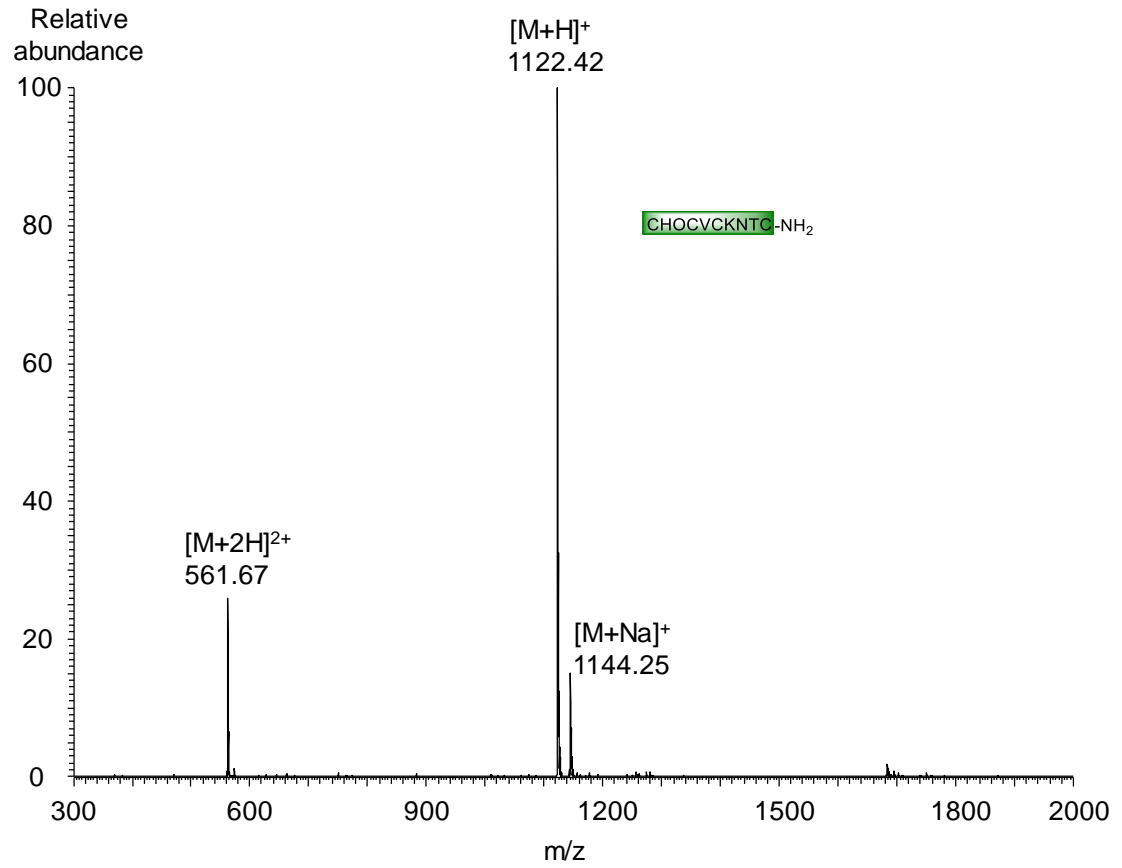

Figure S 70. UPLC-MS analysis of CHOCVCKNTC-NH2. a) LC trace. Eluent A $0.10 \%$ TFA in water, eluent $\mathrm{B} 0.10 \%$ TFA in $\mathrm{CH}_{3} \mathrm{CN}$, ACQUITY UPLC Peptide BEH $300 \AA 1.7 \mu \mathrm{m}(2.1$ $\times 150 \mathrm{~mm}$ ) column, $50{ }^{\circ} \mathrm{C}$, gradient $0-70 \% \mathrm{~B}$ in $20 \mathrm{~min}, 0.4 \mathrm{~mL} / \mathrm{min}$, UV detection. b) MS trace: Calcd. for $[\mathrm{M}+\mathrm{H}]^{+}$(monoisotopic): 1122.42 , found: 1122.42 . 
SetCysCGVONAA-MPA peptide was synthesized on $0.05 \mathrm{mmol}$ scale as described in the general procedure. TFA/ $\mathrm{H}_{2} \mathrm{O} / \mathrm{TIS} /$ thiophenol/thioanisol 87.5:2.5:5:2.5:2.5 (8 mL) was used as the cleavage cocktail. The $\mathrm{SEA}^{\text {on }}$ peptide which was recovered by precipitation from $\mathrm{Et}_{2} \mathrm{O} /$ heptane was immediately converted into the corresponding MPA thioester using the following procedure.

MPA $(0.625 \mathrm{~mL})$ was dissolved in $11.88 \mathrm{~mL}$ of water and the $\mathrm{pH}$ of the solution was adjusted to 4.0 by addition of $6 \mathrm{M} \mathrm{NaOH}$. The SEA ${ }^{\text {on }}$ peptide recovered after acidic cleavage was added to this solution of MPA. Due to the low solubility of the peptide, solid $\mathrm{Gn} \cdot \mathrm{HCl}(1.99 \mathrm{~g})$ was added to the reaction mixture. Once the $\mathrm{pH}$ was readjusted to 4.0 by addition of $6 \mathrm{M} \mathrm{NaOH}$, the reaction was stirred under inert atmosphere at $37{ }^{\circ} \mathrm{C}$ for $7 \mathrm{~h}$. The reaction mixture was then acidified with $\mathrm{AcOH}(1 \mathrm{~mL})$ and extracted with $\mathrm{Et}_{2} \mathrm{O}(5 \times)$ to remove the MPA. Purification of the crude by semi-preparative HPLC (eluent A $0.10 \%$ TFA in water, eluent B $0.10 \%$ TFA in $\mathrm{CH}_{3} \mathrm{CN}, 20 \mathrm{~mL} / \mathrm{min}, 0-10 \%$ eluent $\mathrm{B}$ in $5 \mathrm{~min}$ then $10-25 \%$ in $30 \mathrm{~min}, \mathrm{C} 18$ column, detection at $215 \mathrm{~nm}, 30{ }^{\circ} \mathrm{C}$ ) provided SetCysCGVONAA-MPA peptide as a white solid after lyophilisation ( $7.22 \mathrm{mg}, 14 \%$ overall yield). 
Characterization of SetCysCGVONAA-MPA

a)

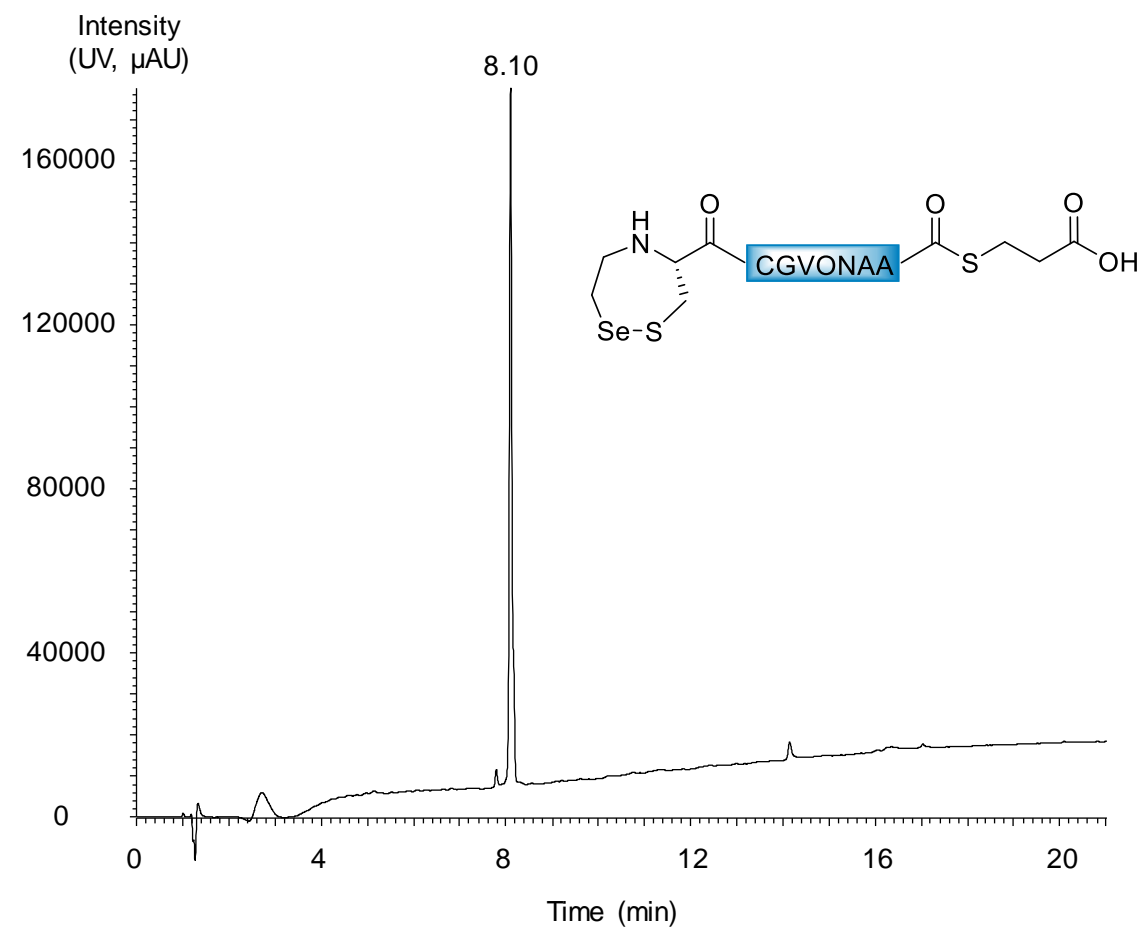

b)

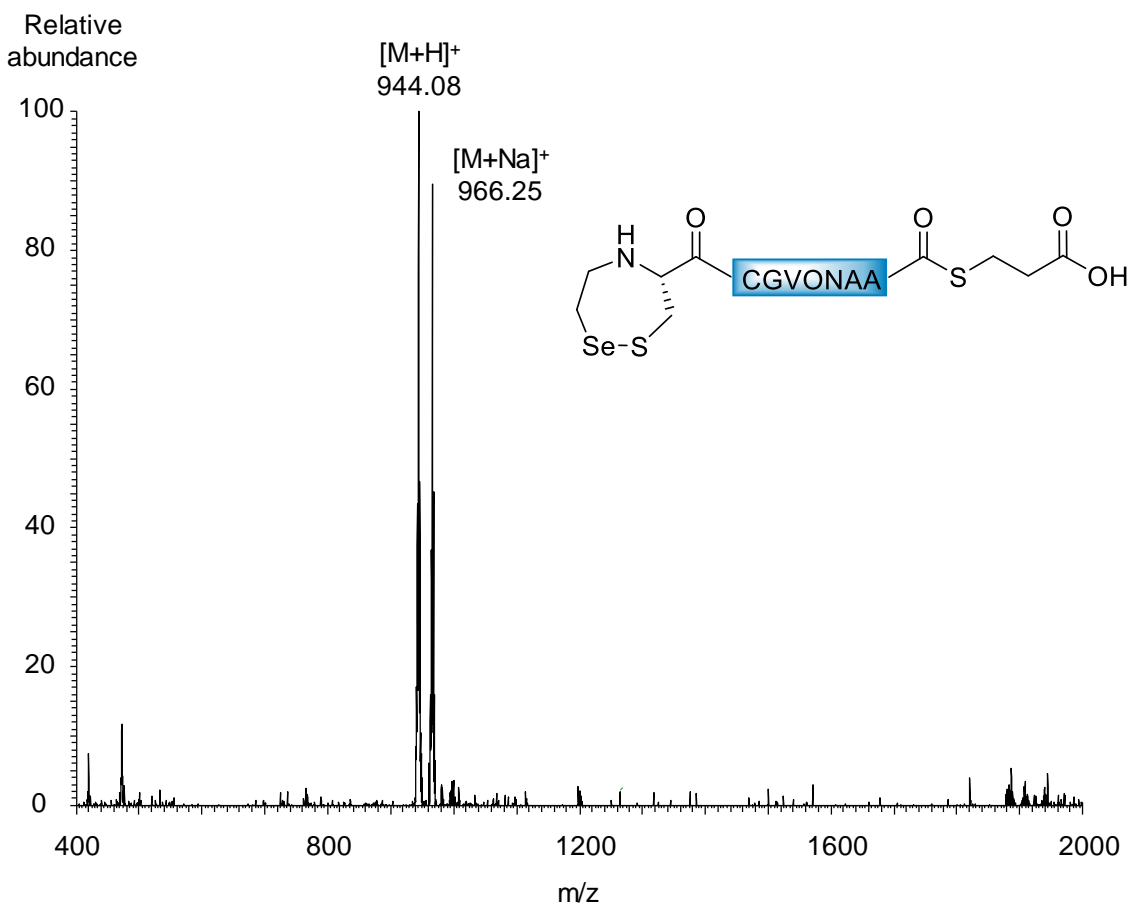

Figure S 71 . UPLC-MS analysis of SetCysCGVONAA-MPA. a) LC trace. Eluent A $0.10 \%$ TFA in water, eluent B $0.10 \%$ TFA in $\mathrm{CH}_{3} \mathrm{CN}$, ACQUITY UPLC Peptide BEH $300 \AA 1.7 \mu \mathrm{m}$ $(2.1 \times 150 \mathrm{~mm})$ column, $50{ }^{\circ} \mathrm{C}$, gradient $0-70 \% \mathrm{~B}$ in $20 \mathrm{~min}, 0.4 \mathrm{~mL} / \mathrm{min}$, UV detection. b) MS trace: Calcd. for $[\mathrm{M}+\mathrm{H}]^{+}$(monoisotopic): 944.21 , found: 944.08 . 
To a solution of $\mathrm{Gn} \cdot \mathrm{HCl}(287 \mathrm{mg})$ in $0.1 \mathrm{M}$, pH 7.4 phosphate buffer $(300 \mu \mathrm{L})$ was added MPAA $(16.8 \mathrm{mg})$ and the $\mathrm{pH}$ of the mixture was adjusted to 7.25 by addition of $6 \mathrm{M} \mathrm{NaOH}$. Peptide CHOCVCKNTC-NH2 $(2.50 \mathrm{mg}, \quad 1.70 \mu \mathrm{mol}, 1$ equiv $)$ and peptide SetCysCGVONAA-MPA $(1.80 \mathrm{mg}, 1.70 \mu \mathrm{mol}, 1$ equiv) were successively dissolved in the MPAA solution $(0.472 \mathrm{~mL})$ and the mixture was stirred at $37^{\circ} \mathrm{C}$. The progress of the ligation leading to the elongated SeCys peptide intermediate was followed by HPLC (Figure S 72).

After completion of the NCL ligation $(2 \mathrm{~h})$, the second step of the process leading to the conversion of the SetCys residue into Cys residue was induced by addition of TCEP. To a solution of $\mathrm{Gn} \cdot \mathrm{HCl}(287 \mathrm{mg})$ in $0.1 \mathrm{M}, \mathrm{pH} 7.4$ phosphate buffer $(300 \mu \mathrm{L})$ were added TCEP. $\mathrm{HCl}(28.6 \mathrm{mg})$, sodium ascorbate $(9.9 \mathrm{mg})$ and MPAA $(16.8 \mathrm{mg})$ and the $\mathrm{pH}$ of the mixture was adjusted to 5.85 by addition of $6 \mathrm{M} \mathrm{NaOH}$. The previous ligation mixture containing the elongated SetCys peptide intermediate was then diluted with the solution of TCEP $(0.472 \mathrm{~mL})$ and the $\mathrm{pH}$ of the new mixture was readjusted to 5.98 by addition of $6 \mathrm{M}$ $\mathrm{HCl}$. The reaction mixture was then stirred at $37^{\circ} \mathrm{C}$ for $24 \mathrm{~h}$. After completion of the reaction (Figure S 72), the mixture was acidified with $7.5 \% \mathrm{AcOH}$ in water $(8 \mathrm{~mL})$ and extracted with $\mathrm{Et}_{2} \mathrm{O}(5 \times)$ to remove the MPAA. The crude was purified by HPLC (eluent $\mathrm{A}: \mathrm{H}_{2} \mathrm{O}$ containing $0.1 \%$ TFA, eluent $\mathrm{B}=$ acetonitrile containing $0.1 \%$ TFA, $0-5 \%$ eluent $\mathrm{B}$ in 5 min then $5-20 \%$ eluent B in $30 \mathrm{~min}, 6 \mathrm{~mL} / \mathrm{min}, \mathrm{C} 18$ column, RT, detection at $215 \mathrm{~nm}$ ) to give the linear sequence of conotoxin OVIA as a white solid after lyophilization (1.48 mg, 40\%).

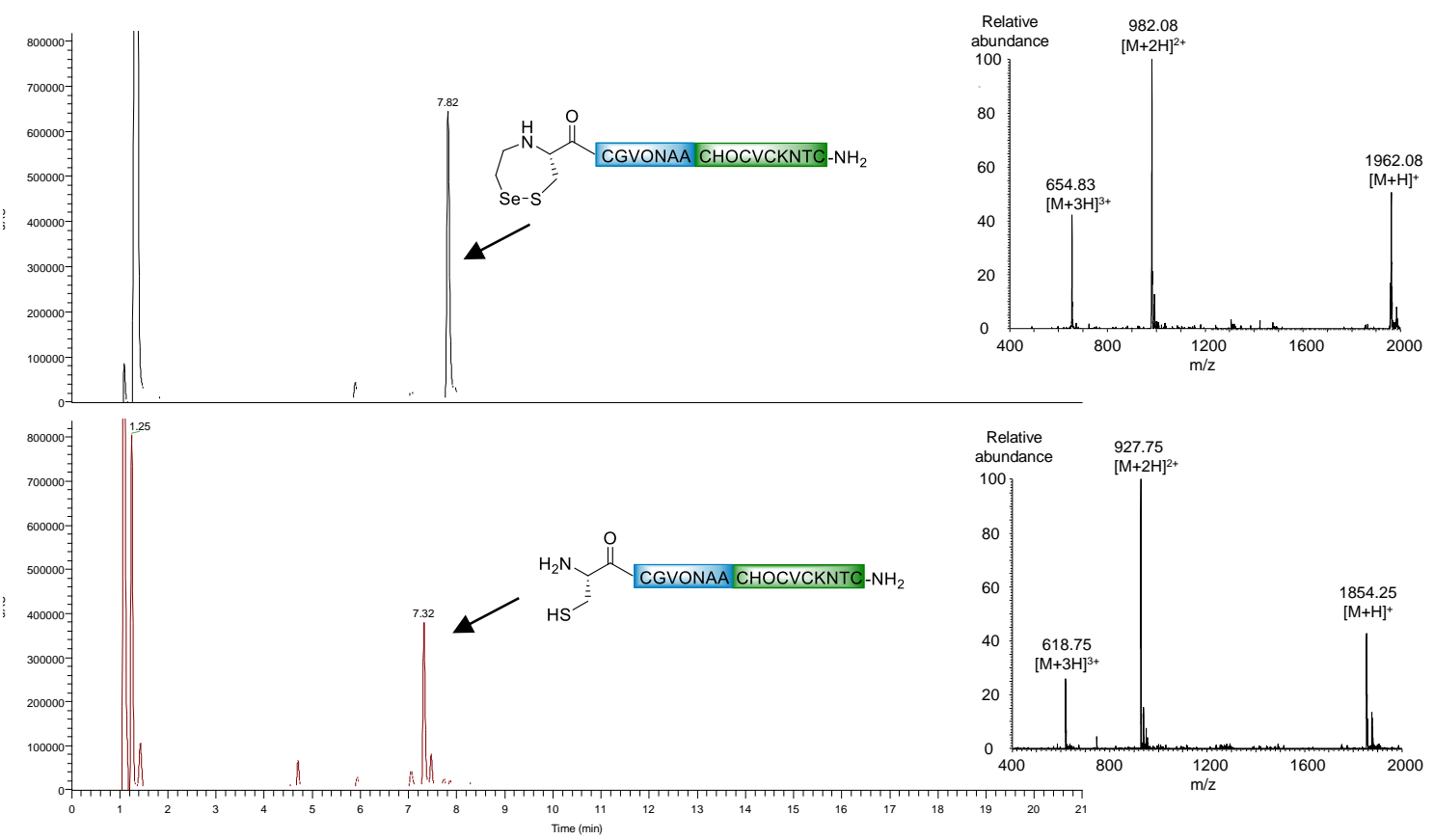

Figure S 72. Conotoxin OIVA assembly monitored by UPLC-MS. a) Analysis of the reaction mixture $2 \mathrm{~h}$ after mixing the two partners of the ligation reaction. The elongated SetCys peptide is detected in mixture with its reduced form, due to the addition of TCEP in the analyzed sample (to reduce MPAA adducts). b) Analysis of the reaction mixture $24 \mathrm{~h}$ after the addition of TCEP and ascorbate. LC trace: eluent A $0.10 \%$ TFA in water, eluent B $0.10 \%$ TFA in $\mathrm{CH}_{3} \mathrm{CN}$, 
ACQUITY UPLC Peptide BEH $300 \AA 1.7 \mu \mathrm{m}(2.1 \times 150 \mathrm{~mm})$ column, $50{ }^{\circ} \mathrm{C}$, gradient $0-70 \%$ $\mathrm{B}$ in $20 \mathrm{~min}, 0.4 \mathrm{~mL} / \mathrm{min}$, UV detection.

Characterization of linear conotoxin OVIA peptide
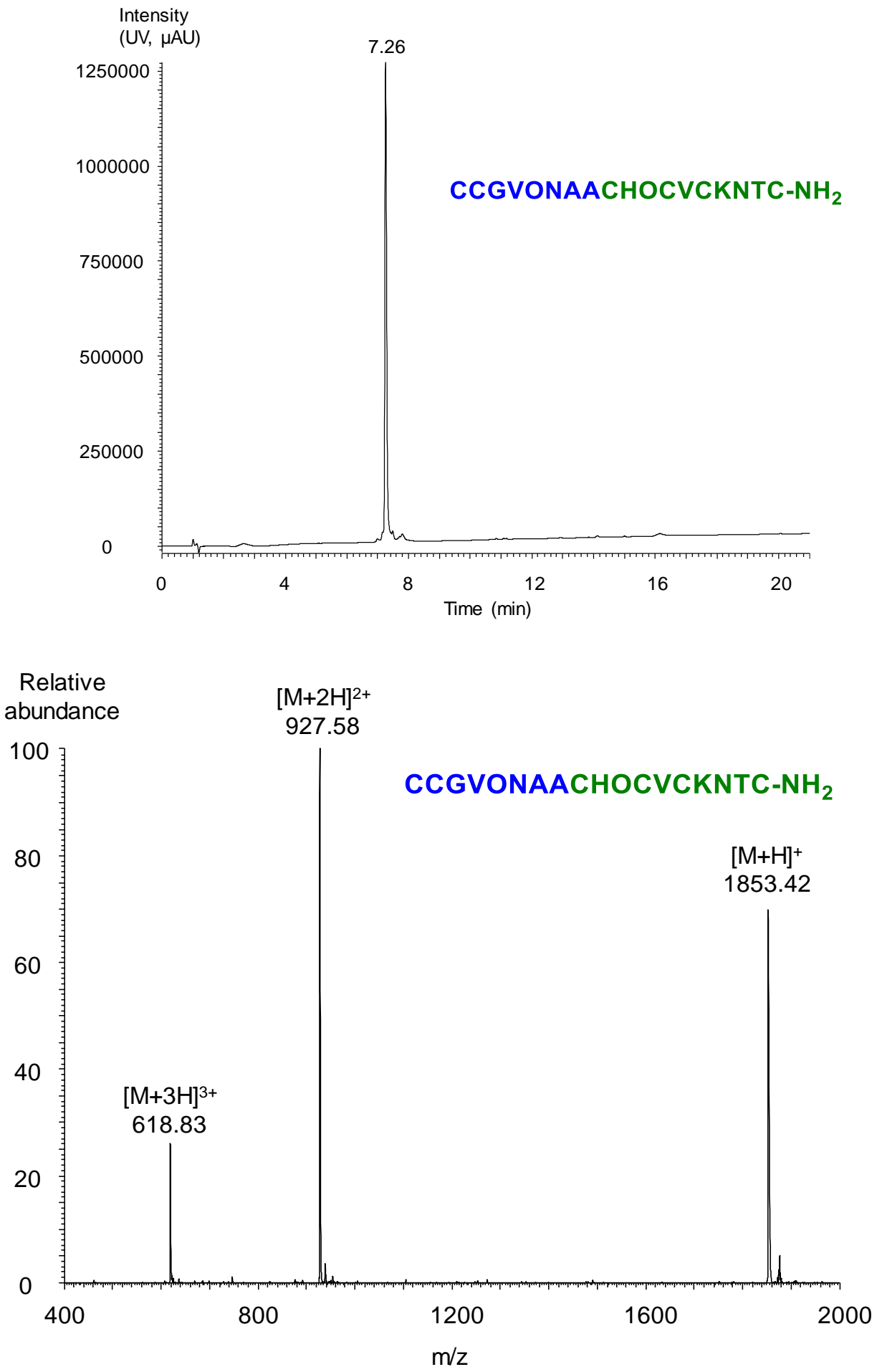
c)

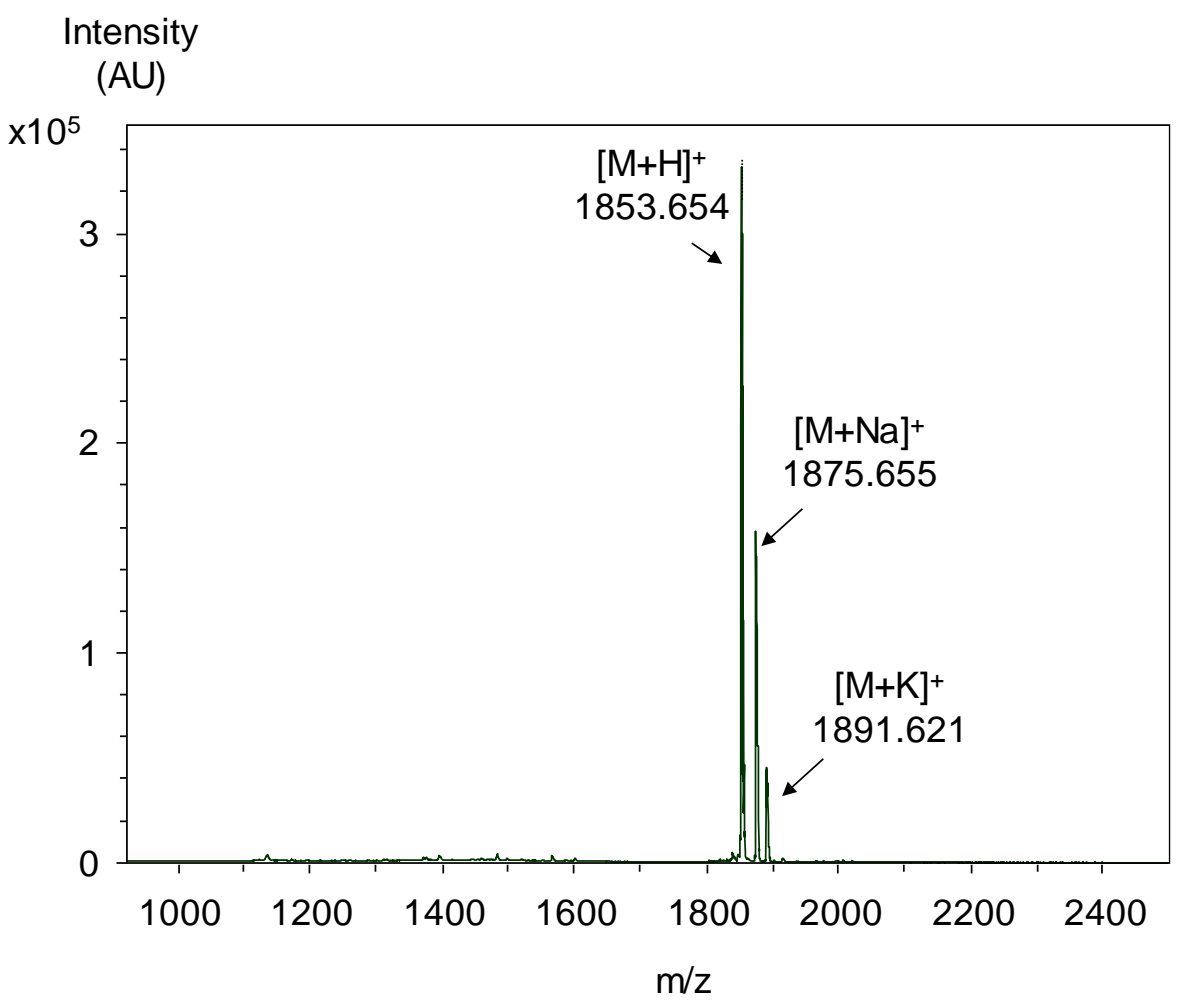

d)

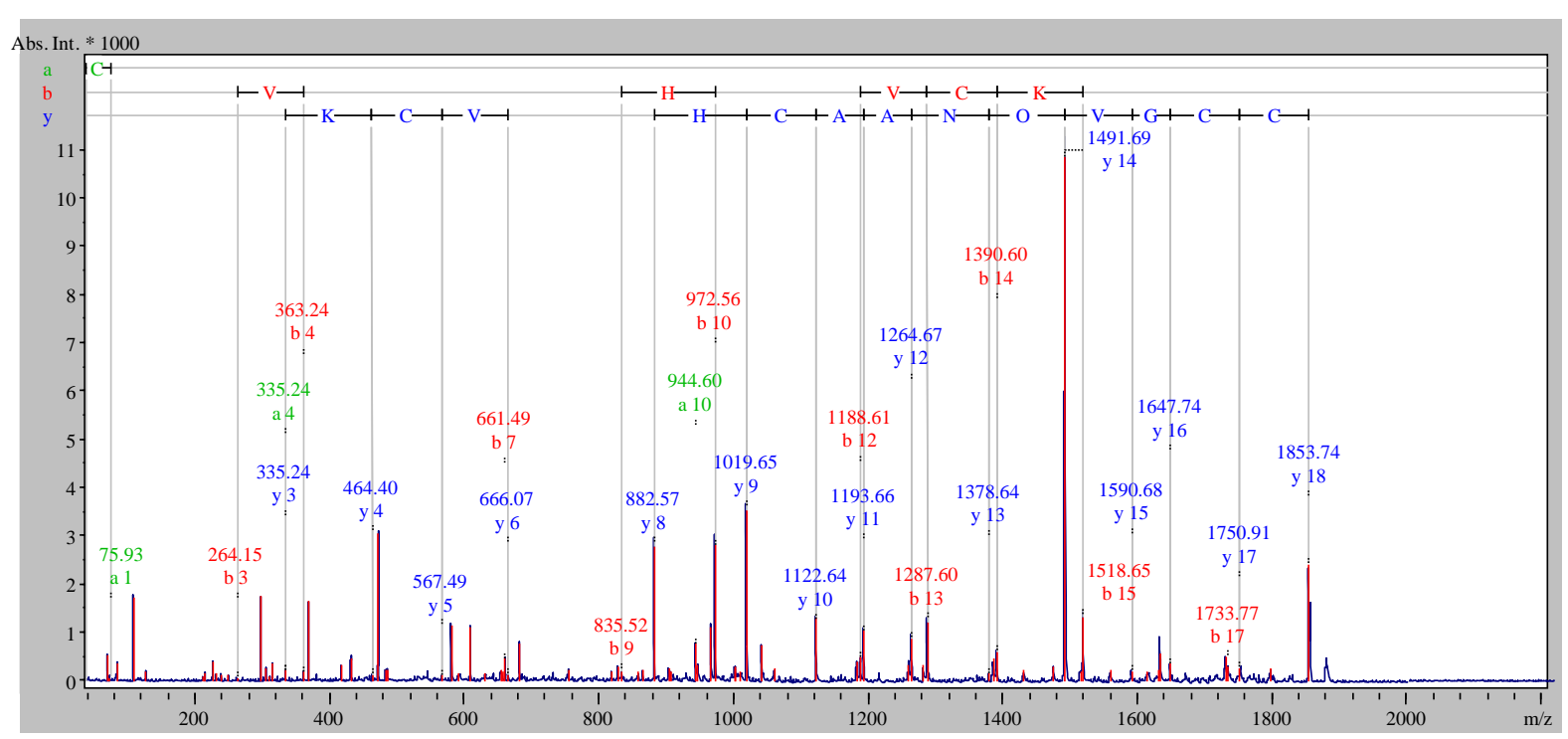

Figure S 73. Analysis of linear conotoxin OIVA. a) LC trace. Eluent A $0.10 \%$ TFA in water, eluent B $0.10 \%$ TFA in $\mathrm{CH}_{3} \mathrm{CN}$, ACQUITY UPLC Peptide BEH $300 \AA 1.7 \mu \mathrm{m}(2.1 \times 150 \mathrm{~mm})$ column, $50{ }^{\circ} \mathrm{C}$, gradient $0-70 \% \mathrm{~B}$ in $20 \mathrm{~min}, 0.4 \mathrm{~mL} / \mathrm{min}$, UV detection. b) MS trace: Calcd. for $[\mathrm{M}+\mathrm{H}]^{+}$(monoisotopic): 1853.70, found: 1853.42. c) MALDI-TOF analysis of conotoxin OIVA linear peptide. Matrix $\alpha$-cyano-4-hydroxycinnamic acid, positive detection mode, calcd. 
for $[\mathrm{M}+\mathrm{H}]^{+}$(monoisotopic): 1853.70, found: 1853.65. d) MALDI-TOF-TOF sequencing of conotoxin OIVA linear peptide. Matrix $\alpha$-cyano-4-hydroxycinnamic acid, positive detection mode. The MS-MS analysis confirms that the Ala-Cys junction formation by NCL as well as the TCEP-induced conversion of the N-terminal SetCys residue into Cys.

\subsection{NCL under strong reducing conditions (peptide alkyl thioester, TCEP/ascorbate, MPAA, property 5)}

When SetCys peptide 1 and peptide thioester $\mathbf{4}$ were reacted in the presence of the TCEP, native peptide 6 was recovered as the only ligation product with $64 \%$ yield (Figure S 74).

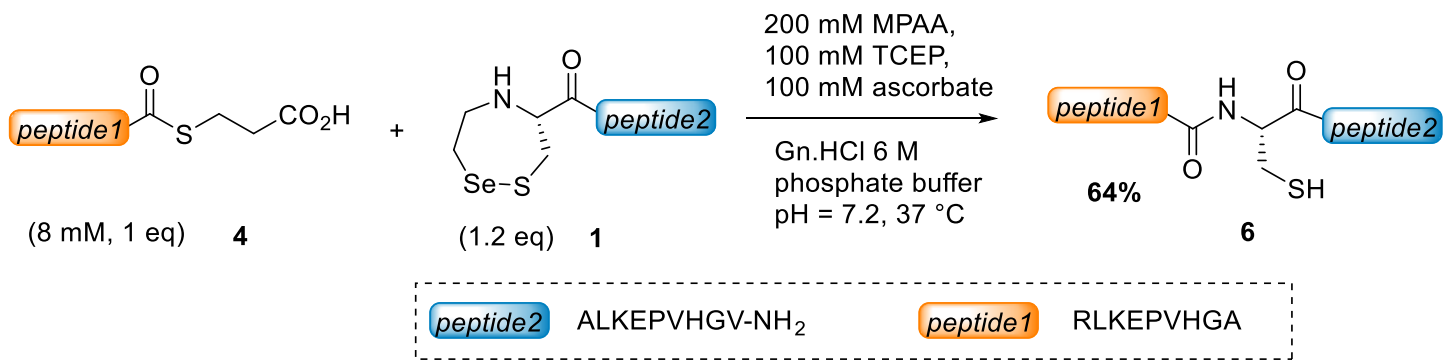

Figure S 74. Model ligation of SetCys peptide 1 and peptidyl MPA-thioester 4 in the presence of TCEP

\section{Protocol}

To a solution of $\mathrm{Gn} \cdot \mathrm{HCl}(574 \mathrm{mg})$ in $0.1 \mathrm{M}$, pH 7.4 phosphate buffer $(600 \mu \mathrm{L})$ were added TCEP.HCl (28.7 mg), sodium ascorbate $(19.8 \mathrm{mg})$ and MPAA $(33.6 \mathrm{mg})$ and the $\mathrm{pH}$ of the mixture was adjusted to 7.17 by addition of $6 \mathrm{M} \mathrm{NaOH}$. RLKEPVHGA-MPA $4(6.04 \mathrm{mg}$, $3.89 \mu \mathrm{mol}, 1 \mathrm{eq})$ and SetCys-ALKEPVHGV-NH2 1 (7.00 mg, $4.67 \mu \mathrm{mol}, 1.2 \mathrm{eq})$ were then successively dissolved in this solution $(212 \mu \mathrm{L})$ and the reaction mixture was stirred at $37^{\circ} \mathrm{C}$ for 4 days. The mixture was then acidified with $5 \% \mathrm{AcOH}$ in water $(10 \mathrm{~mL})$ and extracted with $\mathrm{Et}_{2} \mathrm{O}(5 \times)$ to remove the MPAA. Purification of the crude by HPLC (eluent $\mathrm{A}=$ water containing $0.1 \%$ TFA, eluent $\mathrm{B}=$ acetonitrile in water $4 / 1$ containing $0.1 \% \mathrm{TFA}, 50^{\circ} \mathrm{C}$, detection at $215 \mathrm{~nm}, 3 \mathrm{~mL} / \mathrm{min}, 0-10 \%$ eluent B in $5 \mathrm{~min}$, then 10-25\% eluent B in $30 \mathrm{~min}, \mathrm{C} 18 \mathrm{XBridge}$ column) provided RLKEPVHGACALKEPVHGV-NH2 $\mathbf{6}$ as a white solid after lyophilisation(6.81 mg, 64\%). 


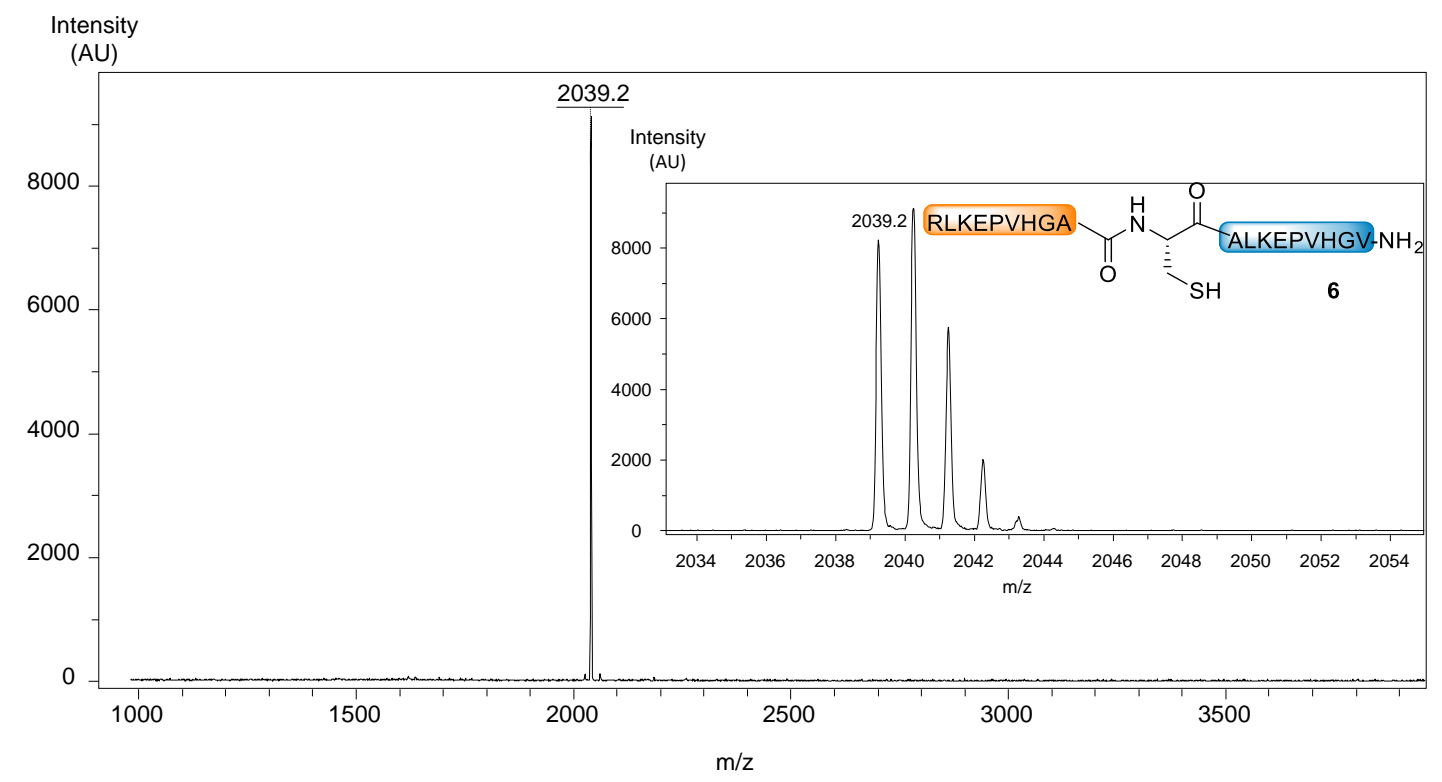

Figure S 75. MALDI-TOF analysis of RLKEPVHGA-CALKEPVHGV-NH2 peptide 6. Matrix $\alpha$-cyano-4-hydroxycinnamic acid, positive detection mode, calcd. for $[\mathrm{M}+\mathrm{H}]^{+}$ (monoisotopic): 2039.13, found: 2039.2.

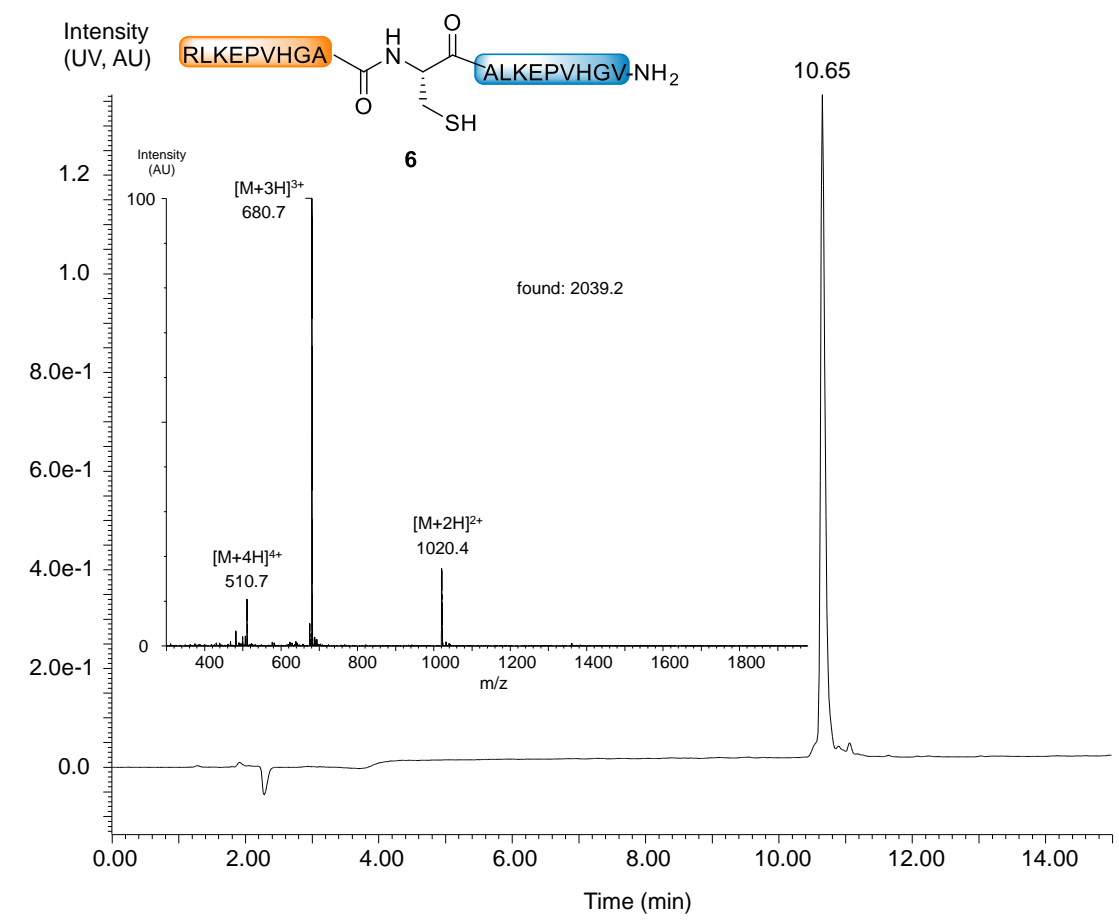

Figure S 76. LC-MS analysis of RLKEPVHGA-CALKEPVHGV-NH2 peptide 6. LC trace: eluent A $0.10 \%$ TFA in water, eluent B $0.10 \%$ TFA in $\mathrm{CH}_{3} \mathrm{CN} /$ water: $4 / 1$ by vol. $\mathrm{C} 18$ Xbridge $\mathrm{BEH} 300 \AA 5 \mathrm{~m}(4.6 \times 250 \mathrm{~mm})$ column, gradient $0-50 \% \mathrm{~B}$ in $15 \mathrm{~min}, 30{ }^{\circ} \mathrm{C}, 1 \mathrm{~mL} / \mathrm{min}, \mathrm{UV}$ detection. MS trace: $\mathrm{m} / \mathrm{z}=1020.4\left([\mathrm{M}+2 \mathrm{H}]^{2+}\right), 680.7\left([\mathrm{M}+3 \mathrm{H}]^{3+}\right), 510.7\left([\mathrm{M}+4 \mathrm{H}]^{4+}\right)$; calcd. for M (average): 2039.4, found 2039.2. 
The presence of the newly formed cysteine residue into the peptide sequence was confirmed by MS-MS analysis (Figure S 77).

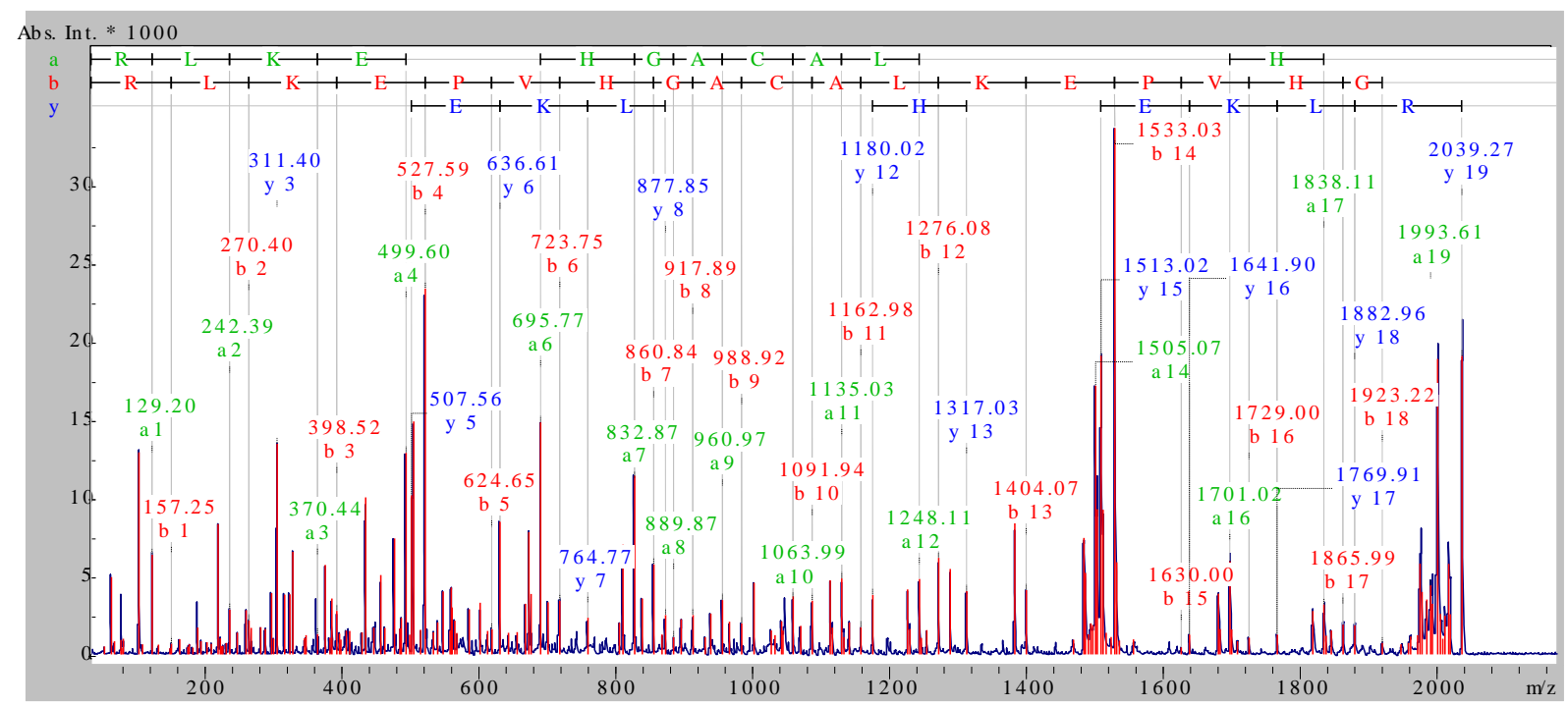

Figure $\mathbf{S}$ 77. MALDI-TOF-TOF sequencing of RLKEPVHGA-CALKEPVHGV-NH2 peptide 6. Matrix $\alpha$-cyano-4-hydroxycinnamic acid, positive detection mode. The MS-MS analysis confirms the presence of Cys residue in the sequence of the peptide (in place of the SetCys amino acid).

The determination of the optical purity for the Ala residue involved in the newly formed junction was performed by chiral GC-MS analysis after acid hydrolysis in deuterated acid. This analysis performed by the compagny C.A.T. $\mathrm{GmbH} \&$ Co Chromatographie und Analysentechnik KG indicated that the Ala residue did not epimerize to a significant extent during the NCL: $1.69 \%$ D-Ala content was measured in the isolated peptide 6.

Besides peptide 6, RLKEPVHGA-OH (0.97 mg, Figure S 78) and CALKEPVHGV-NH2 (1.12 $\mathrm{mg}$, Figure $\mathrm{S}$ 79) were isolated as side products from the reaction mixture. Peptide RLKEPVHGA-OH results from the partial hydrolysis of the peptidyl MPA-thioester 4 whereas the cysteinyl peptide CALKEPVHGV-NH2 results from the decomposition of the unreacted SetCys peptide $\mathbf{1}$. 

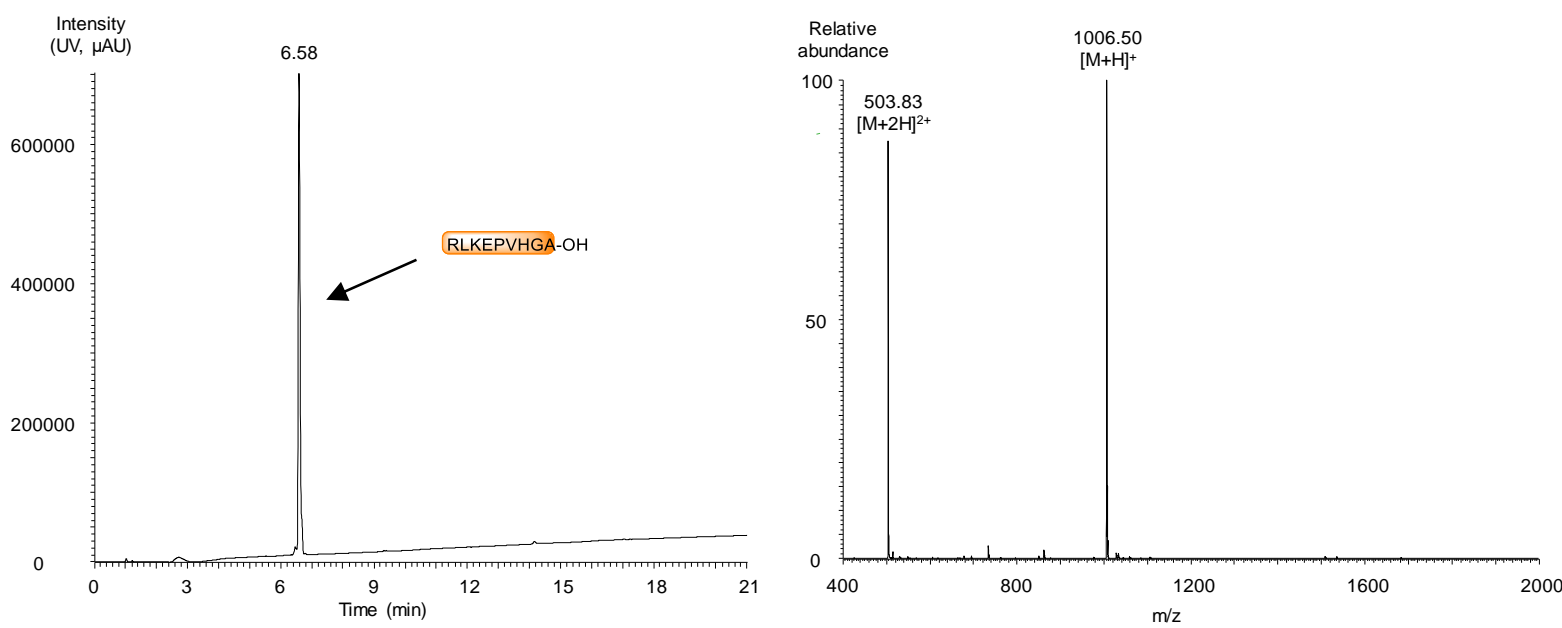

Figure S 78. LC-MS analysis of RLKEPVHGA-OH. a) LC trace. Eluent A $0.10 \%$ TFA in water, eluent B $0.10 \%$ TFA in $\mathrm{CH}_{3} \mathrm{CN}$, ACQUITY UPLC Peptide BEH $300 \AA 1.7 \mu \mathrm{m}(2.1 \times$ $150 \mathrm{~mm}$ ) column, $50{ }^{\circ} \mathrm{C}$, gradient $0-70 \%$ B in $20 \mathrm{~min}, 0.4 \mathrm{~mL} / \mathrm{min}$, UV detection at $215 \mathrm{~mm}$. b) MS trace: calcd. for $[\mathrm{M}+\mathrm{H}]^{+}$(monoisotopic): 1006.57, found: 1006.50 . 
Characterization of peptide CALKEPVHGV-NH :
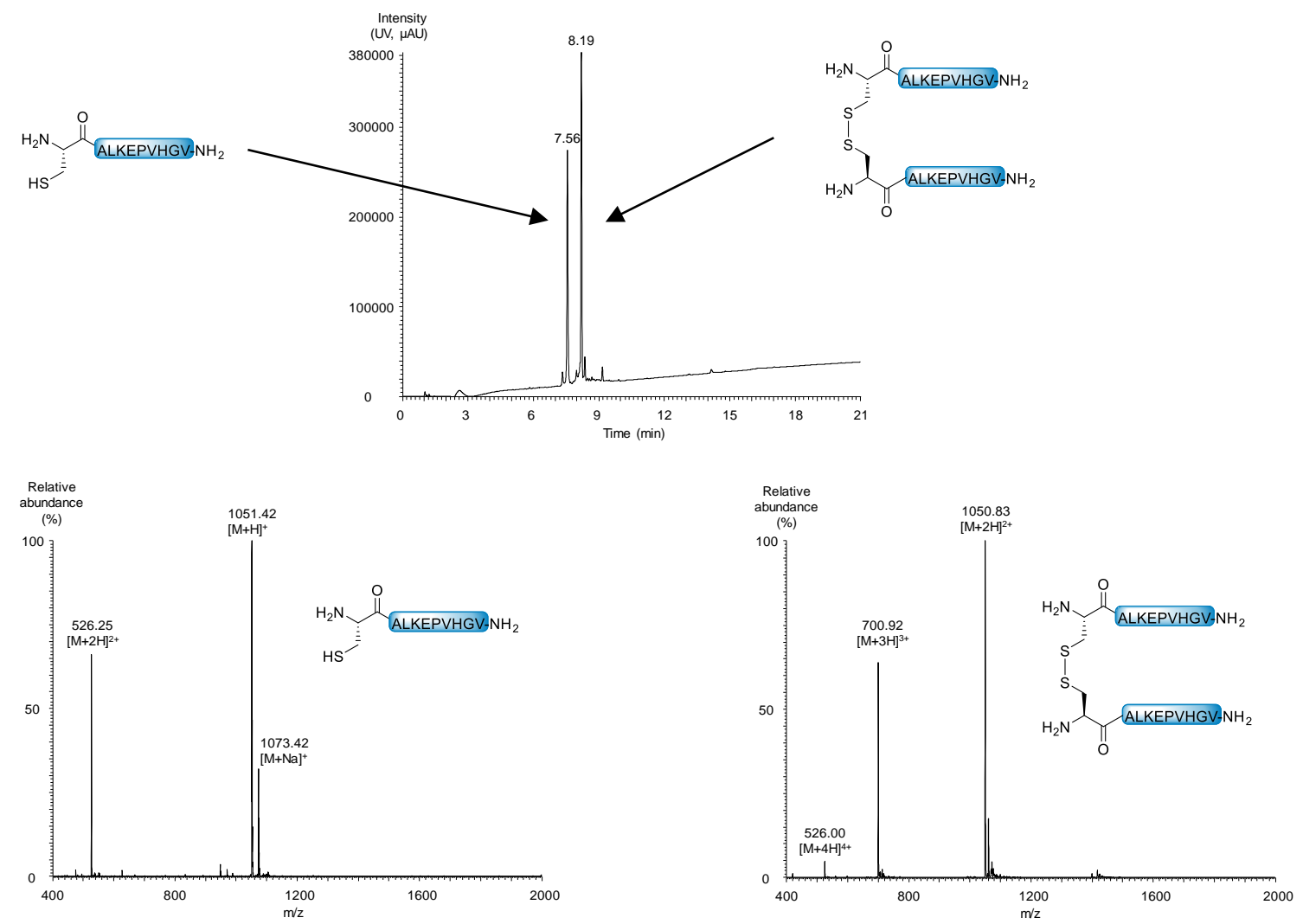

Figure S 79. UPLC-MS analysis of CALKEPVHGV-NH 2 . The peptide was isolated as a mixture of monomer and oxidazed dimer. a) LC trace. Eluent A $0.10 \%$ TFA in water, eluent B $0.10 \%$ TFA in $\mathrm{CH}_{3} \mathrm{CN}$, ACQiITY UPLC Peptide BEH $300 \AA 1.7 \mu \mathrm{m}(2.1 \times 150 \mathrm{~mm})$ column, $50{ }^{\circ} \mathrm{C}$, gradient $0-70 \% \mathrm{~B}$ in $20 \mathrm{~min}, 0.4 \mathrm{~mL} / \mathrm{min}$, UV detection at $215 \mathrm{~nm}$. b) MS trace. For the monomer, calcd. for $[\mathrm{M}+\mathrm{H}]^{+}$(monoisotopic): 1051.56, found: 1051.42 . For the dimer, $\mathrm{m} / \mathrm{z}=$ $1050.83\left([\mathrm{M}+2 \mathrm{H}]^{2+}\right), 700.92\left([\mathrm{M}+3 \mathrm{H}]^{3+}\right), 526.00\left([\mathrm{M}+4 \mathrm{H}]^{4+}\right)$; calcd. for $\mathrm{M}$ (average): 2100.54 , found: 2099.81 . 


\subsection{NCL under strong reducing conditions (peptide aryl thioester, TCEP/ascorbate, no}

added MPAA, property 5)

When SetCys peptide 1 and peptidyl MPAA-thioester ILKEPWHGA-MPAA were reacted in the presence of the TCEP, peptide ILKEPWHGA-CALKEPVHGV-NH2 was recovered as the only ligation product with $40 \%$ isolated yield (Figure S 80 ).

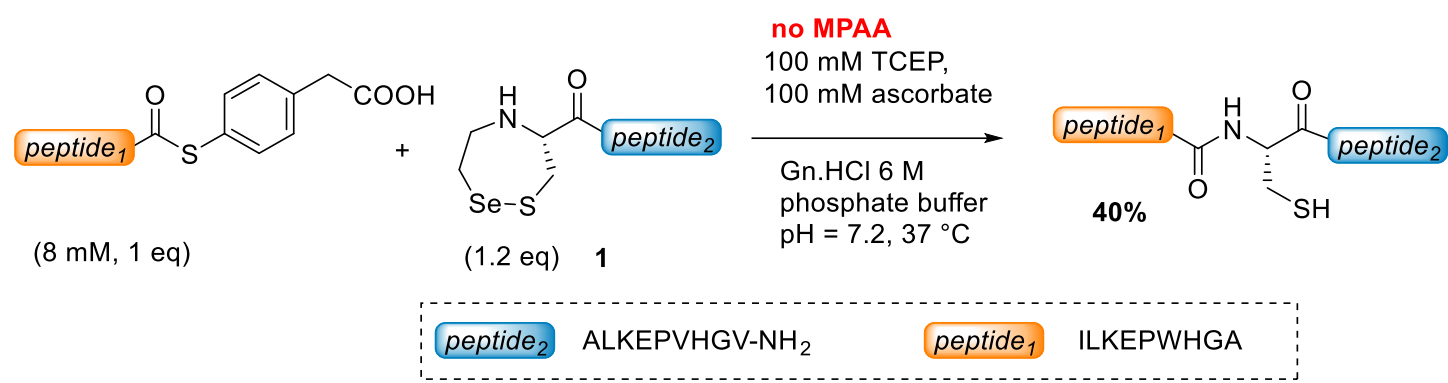

Figure S 80. MPAA-free model ligation of SetCys peptide 1 and peptidyl MPAA-thioester ILKEPWHGA-MPAA in the presence of TCEP.

Synthesis and characterization of ILKEPWHGA-MPAA peptide

ILKEPWHGA-MPAA peptide was synthesized from the corresponding peptide hydrazide using Liu's method. ${ }^{7}$

a)

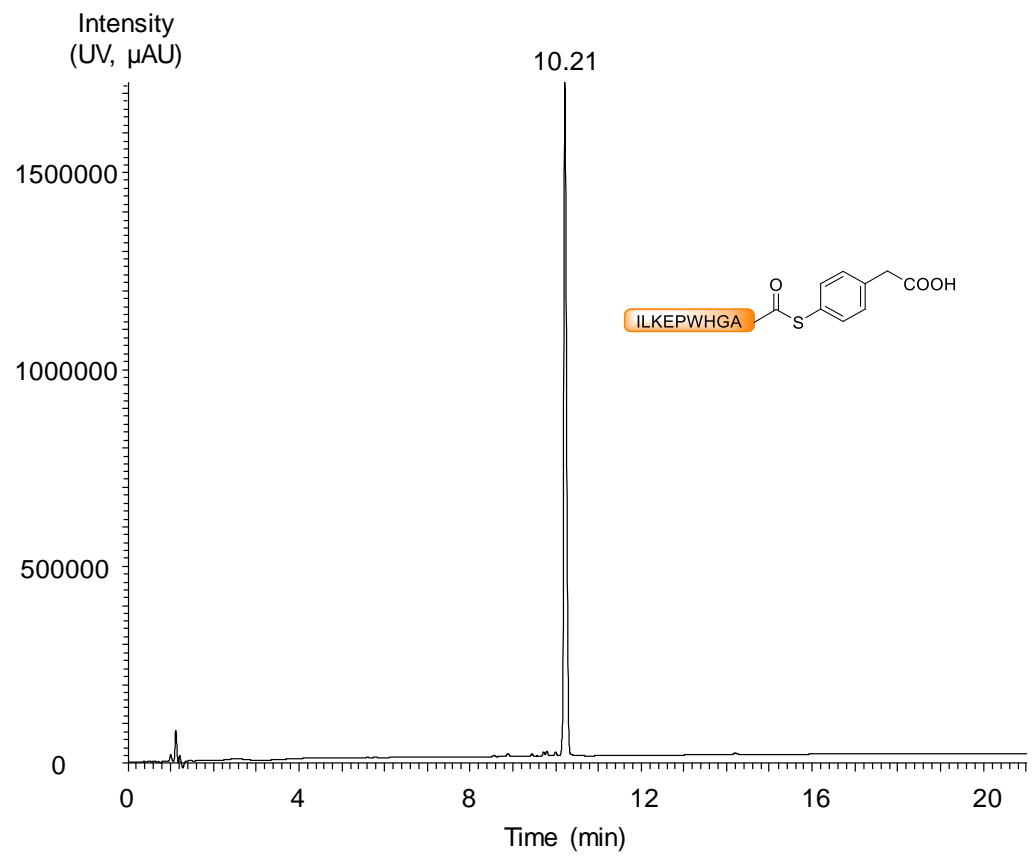


b)

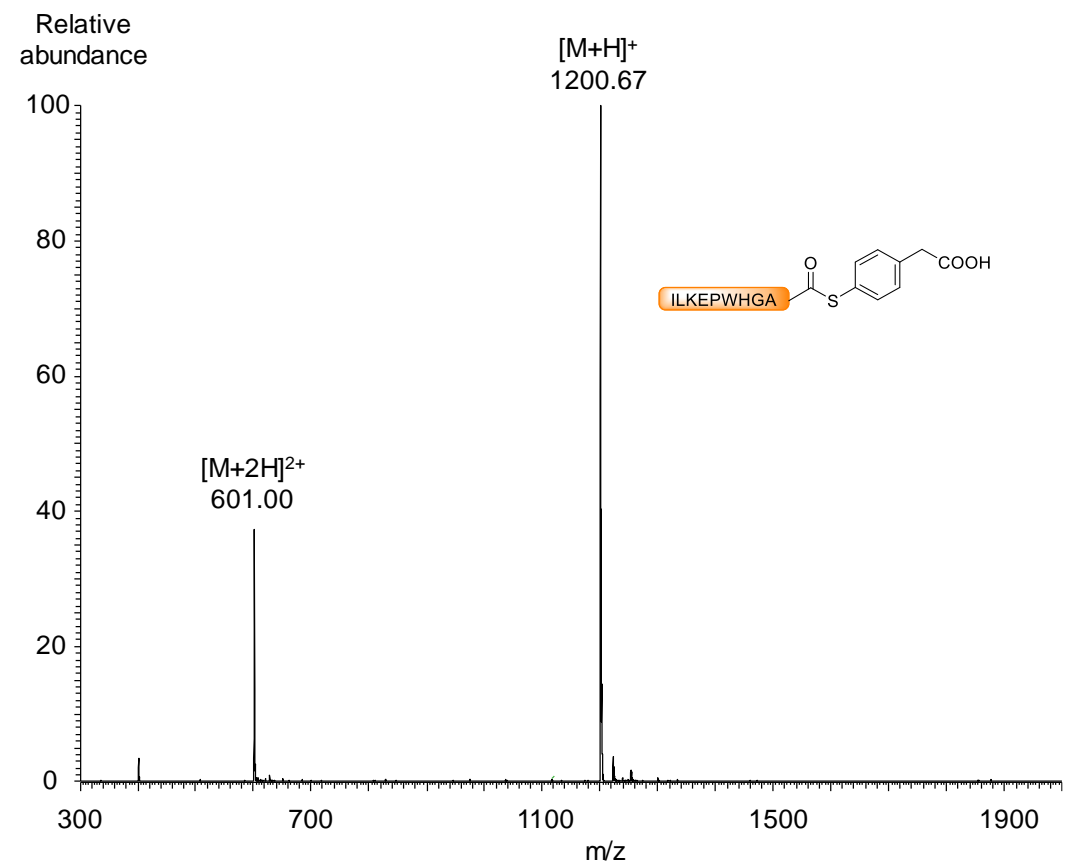

Figure S 81. UPLC-MS analysis of ILKEPWHGA-MPAA. a) LC trace. Eluent A $0.10 \%$ TFA in water, eluent B $0.10 \%$ TFA in $\mathrm{CH}_{3} \mathrm{CN}$, ACQUITY UPLC Peptide BEH $300 \AA 1.7 \mu \mathrm{m}(2.1$ $\times 150 \mathrm{~mm}$ ) column, $50{ }^{\circ} \mathrm{C}$, gradient $0-70 \% \mathrm{~B}$ in $20 \mathrm{~min}, 0.4 \mathrm{~mL} / \mathrm{min}$, UV detection. b) $\mathrm{MS}$ trace:. Calcd. for $[\mathrm{M}+\mathrm{H}]^{+}$(monoisotopic): 1200.58 , found: 1200.67 .

\section{Protocol}

To a solution of $\mathrm{Gn} \cdot \mathrm{HCl}(287 \mathrm{mg})$ in $0.1 \mathrm{M}$, pH 7.4 phosphate buffer $(300 \mu \mathrm{L})$ were added sodium ascorbate $(9.9 \mathrm{mg})$ and TCEP. $\mathrm{HCl}(14.3 \mathrm{mg})$ and the $\mathrm{pH}$ of the mixture was adjusted to 7.27 by addition of $6 \mathrm{M} \mathrm{NaOH}$. SetCys peptide 1 (2.00 mg, $1.33 \mu \mathrm{mol}, 1.2 \mathrm{eq})$ and peptidyl MPAA-thioester ILKEPWHGA-MPAA $(1.71 \mathrm{mg}, 1.10 \mu \mathrm{mol}, 1 \mathrm{eq})$ were then successively dissolved in this solution $(139 \mu \mathrm{L})$ and the reaction mixture was stirred at $37{ }^{\circ} \mathrm{C}$. The mixture was then acidified with $7.5 \% \mathrm{AcOH}$ in water $(8 \mathrm{~mL})$ and extracted with $\mathrm{Et}_{2} \mathrm{O}(5 \times)$. Purification of the crude by HPLC (eluent $\mathrm{A}=$ water containing $0.1 \%$ TFA, eluent $\mathrm{B}=$ acetonitrile containing $0.1 \%$ TFA, gradient: $0-10 \%$ eluent B in 5 min then $10-25 \%$ in $25 \mathrm{~min}, 6 \mathrm{~mL} / \mathrm{min}$, C18 column, RT, detection at $215 \mathrm{~nm}$,) provided ILKEPWHGA-CALKEPVHGV-NH2 peptide as a white solid after lyophilisation $(1.17 \mathrm{mg}, 40 \%)$. 
a)

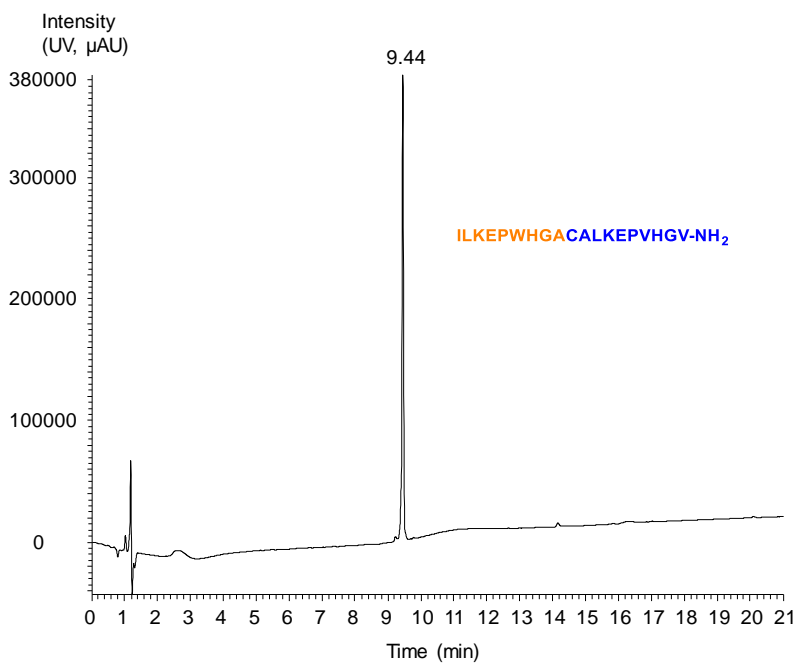

b)

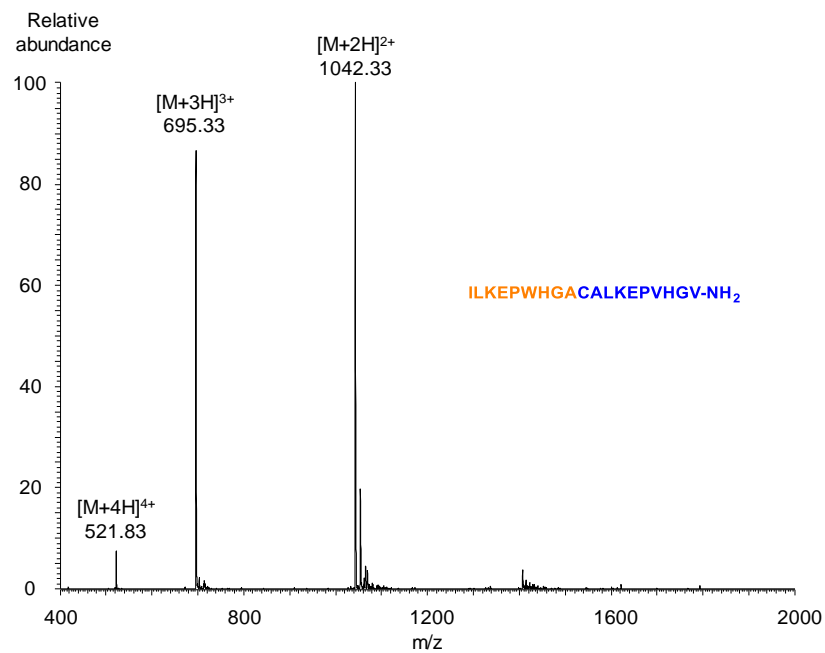

Figure S 82. UPLC-MS analysis of ILKEPWHGACALKEPVHGV-NH2. a) LC trace. Eluent A $0.10 \%$ TFA in water, eluent B $0.10 \%$ TFA in $\mathrm{CH}_{3} \mathrm{CN}$, ACQUITY UPLC Peptide BEH 300 $\AA 1.7 \mu \mathrm{m}(2.1 \times 150 \mathrm{~mm})$ column, $50{ }^{\circ} \mathrm{C}$, gradient $0-70 \% \mathrm{~B}$ in $20 \mathrm{~min}, 0.4 \mathrm{~mL} / \mathrm{min}, \mathrm{UV}$ detection. b) MS trace: $\mathrm{m} / \mathrm{z}=1853.42\left([\mathrm{M}+2 \mathrm{H}]^{2+}\right), 927.58\left([\mathrm{M}+3 \mathrm{H}]^{3+}\right), 618.83\left([\mathrm{M}+4 \mathrm{H}]^{4+}\right)$. Calcd. for M (average): 2083.49, found: 2082.99. 
Monitoring of the reaction by UPLC-MS (Figure S 83) shows the transient accumulation of the mono- and di-ligated species $\mathrm{A}$ and $\mathrm{B}$, the latter being quickly converted into the mono-ligated compounds A. The intermediate $\mathrm{A}$ is then slowly converted into the final peptide ILKEPWHGACALKEPVHGV-NH2 featuring a cysteine as a junction residue.

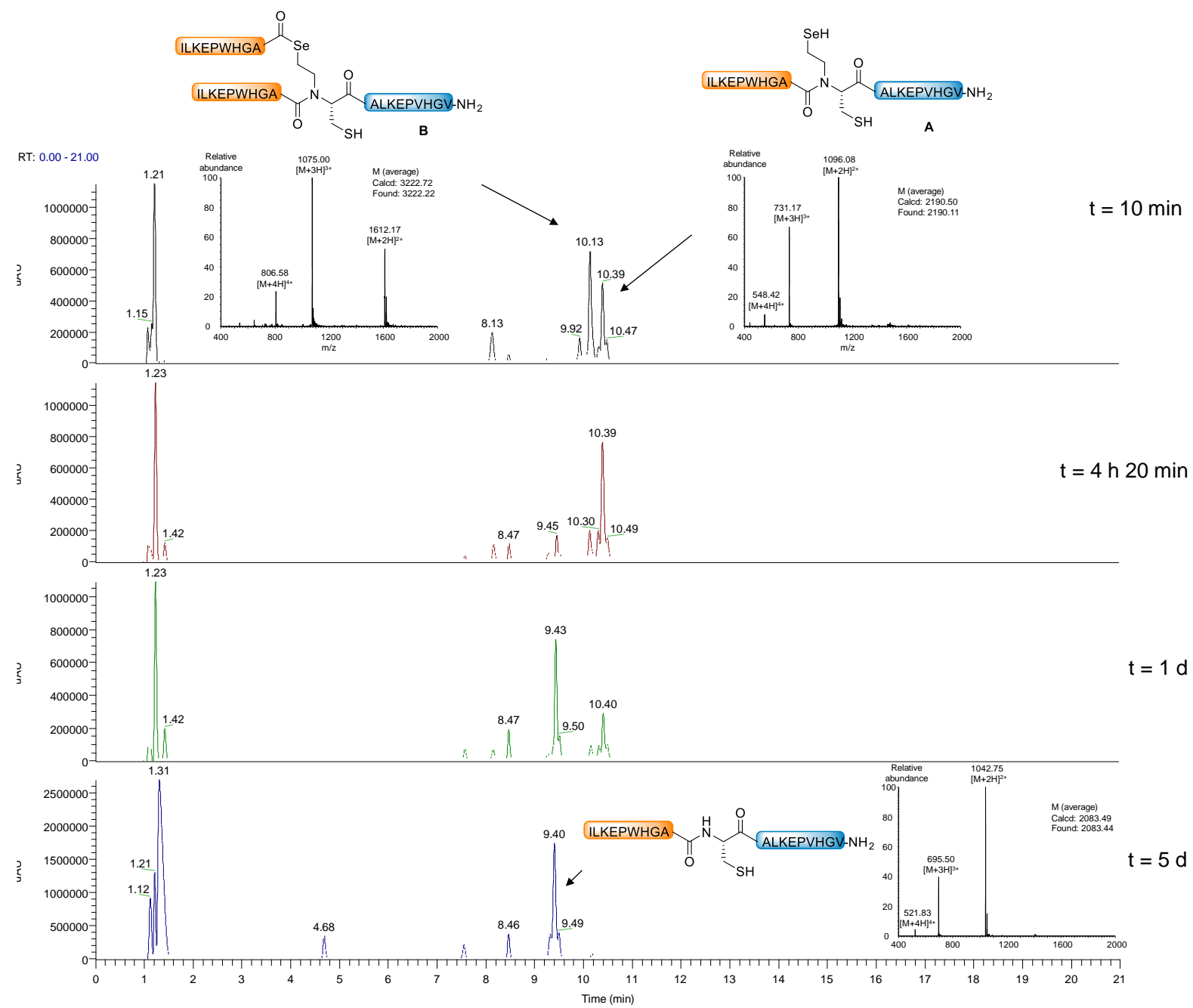

Figure S 83. UPLC-MS monitoring of the reaction between SetCys peptide 1 and peptidyl MPAA-thioester ILKEPWHGA-MPAA performed in the presence of TCEP. 


\subsection{NCL under strong reducing conditions (peptide alkyl thioester, DTT, MPAA, property 5)}

When SetCys peptide $\mathbf{1}$ and peptide thioester $\mathbf{4}$ were reacted in the presence of the DTT, native peptide 6 was produced with 55\% yield (Figure S 84).

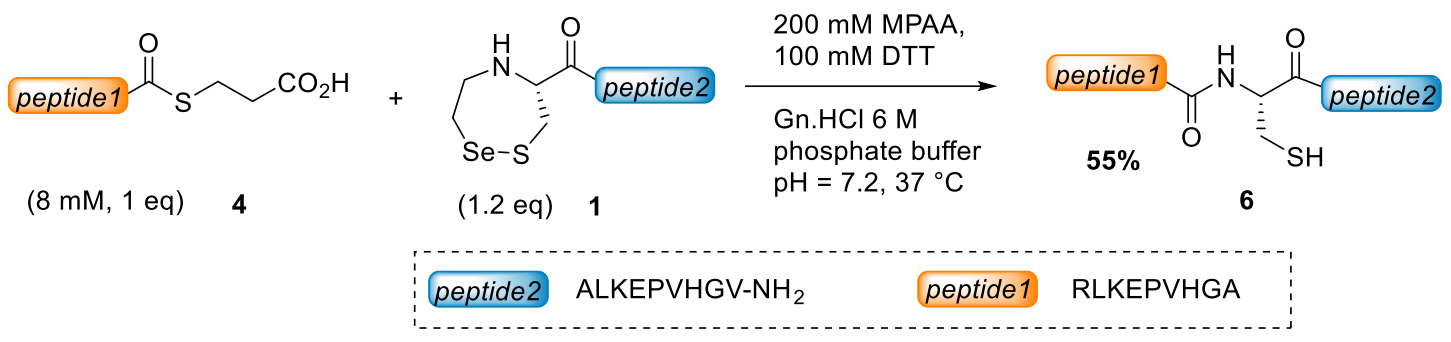

Figure S 84. Ligation of SetCys peptide 1 and peptide thioester 4 in the presence of DTT.

\section{Protocol}

To a solution of $\mathrm{Gn} \cdot \mathrm{HCl}(574 \mathrm{mg})$ in $\mathrm{pH} 7.40 .1 \mathrm{M}$ phosphate buffer $(600 \mu \mathrm{L})$ were added DTT $(15.4 \mathrm{mg})$ and MPAA $(33.6 \mathrm{mg})$ and the $\mathrm{pH}$ of the mixture was adjusted to 7.24 by addition of $6 \mathrm{M} \mathrm{NaOH}$. Peptide thioester 4 (6.04 mg, $3.89 \mu \mathrm{mol}, 1 \mathrm{eq})$ and SetCys peptide 1 (7.00 mg, 4.67 $\mu \mathrm{mol}, 1.2 \mathrm{eq})$ were then successively dissolved in this solution $(487 \mu \mathrm{L})$ and the reaction mixture was stirred at $37{ }^{\circ} \mathrm{C}$. The reaction was monitored by HPLC (Figure S 85). After completion of the reaction (4 days), the mixture was then acidified with $10 \% \mathrm{AcOH}$ in water $(8 \mathrm{~mL})$ and extracted with $\mathrm{Et}_{2} \mathrm{O}(5 \times)$ to remove the MPAA. Purification of the crude by HPLC (eluent A: water containing $0.1 \%$ TFA, eluent B: acetonitrile containing $0.1 \%$ TFA, gradient: $0-10 \%$ eluent B in $5 \mathrm{~min}$ then 10-30\% eluent B in $30 \mathrm{~min}, 6 \mathrm{~mL} / \mathrm{min}, \mathrm{C} 18$ column, rt, detection at $215 \mathrm{~nm}$ ) provided peptide 6 as a white solid after lyophilisation $(5.83 \mathrm{mg}, 55 \%)$. 
a) $t=10 \mathrm{~min}$
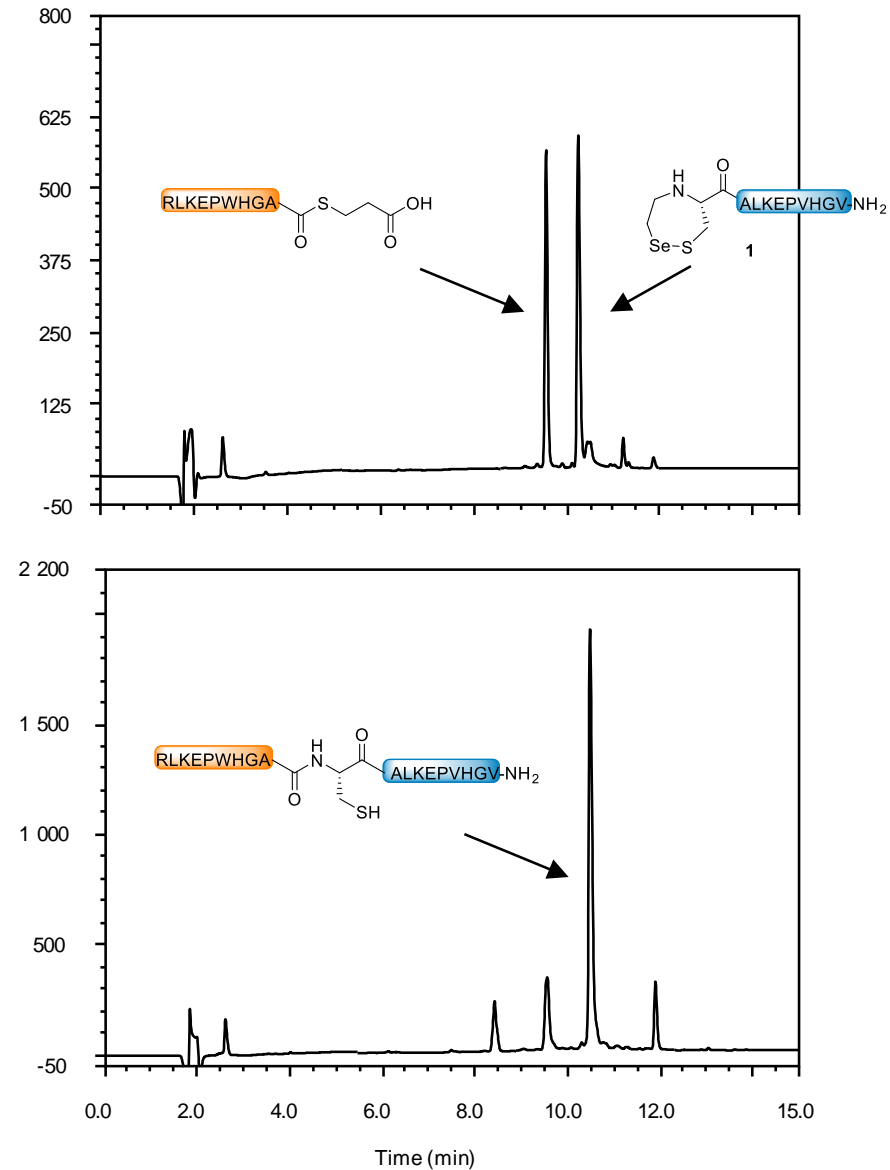

Figure S 85. HPLC-MS monitoring of the reaction between SetCys peptide 1 and peptide thioester 4 performed in the presence of DTT. LC trace: eluent A $0.10 \%$ TFA in water, eluent B $0.10 \%$ TFA in $\mathrm{CH}_{3} \mathrm{CN} /$ water: $4 / 1$ by vol. $\mathrm{C} 18$ Xbridge BEH $300 \AA 5 \mu \mathrm{m}(4.6 \times 250 \mathrm{~mm})$ column, gradient $0-50 \% \mathrm{~B}$ in $15 \mathrm{~min}, 30{ }^{\circ} \mathrm{C}, 1 \mathrm{~mL} / \mathrm{min}$, UV detection $(215 \mathrm{~nm})$. 


\section{Stability and reactivity of $\mathrm{N}$-(2-sulfanylethyl)Cys (SutCys) unit as a function of the reducing power of the reaction mixture (Figure $\mathbf{2 b}$ )}

The SutCys-ALKEPVHGV-NH2 peptide used as model compound in this study was synthesized according to Ruff et al. ${ }^{8}$

\subsection{Stability under strong reducing conditions}

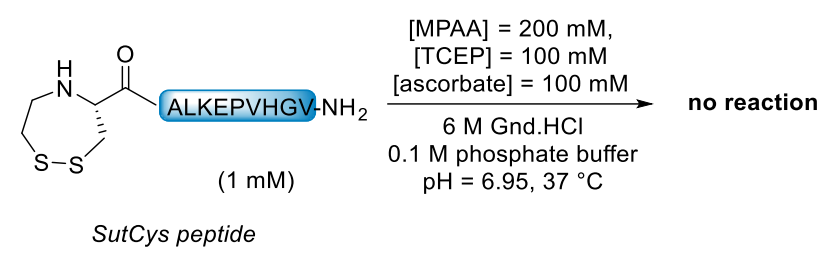

Figure S 86: Stability of the SutCys-ALKEPVHGV-NH2 peptide under strong reductive conditions.

To a solution of $\mathrm{Gn} \cdot \mathrm{HCl}(287 \mathrm{mg})$ in $0.1 \mathrm{M}$ phosphate buffer $(300 \mu \mathrm{L})$ were added TCEP.HCl $(14.3 \mathrm{mg})$, sodium ascorbate $(9.9 \mathrm{mg})$ and MPAA $(16.8 \mathrm{mg})$ and the $\mathrm{pH}$ of the mixture was adjusted to 6.95 by addition of $6 \mathrm{M} \mathrm{NaOH}$. SutCys-ALKEPVHGV-NH2 peptide $(0.23 \mathrm{mg}$, $0.16 \mu \mathrm{mol})$ was then dissolved in this solution $(160 \mu \mathrm{L})$ and the reaction mixture was stirred at $37^{\circ} \mathrm{C}$.

The reaction was monitored by HPLC (Figure S 87). For each point, an $8 \mu \mathrm{L}$ aliquot was taken from the reaction mixture and quenched by adding $10 \% \mathrm{AcOH}$ in water $(100 \mu \mathrm{L})$. The sample was then extracted with $\mathrm{Et}_{2} \mathrm{O}$ to remove MPAA prior to HPLC analysis.

The HPLC monitoring showed that the SutCys residue didn't lose its sulfanylethyl arm, even after several days of incubation in presence of TCEP. Neither increasing (from 6.95 to 7.92) nor decreasing (from 6.95 to 6.06) the $\mathrm{pH}$ of the reaction mixture changed the outcome of the reaction. 


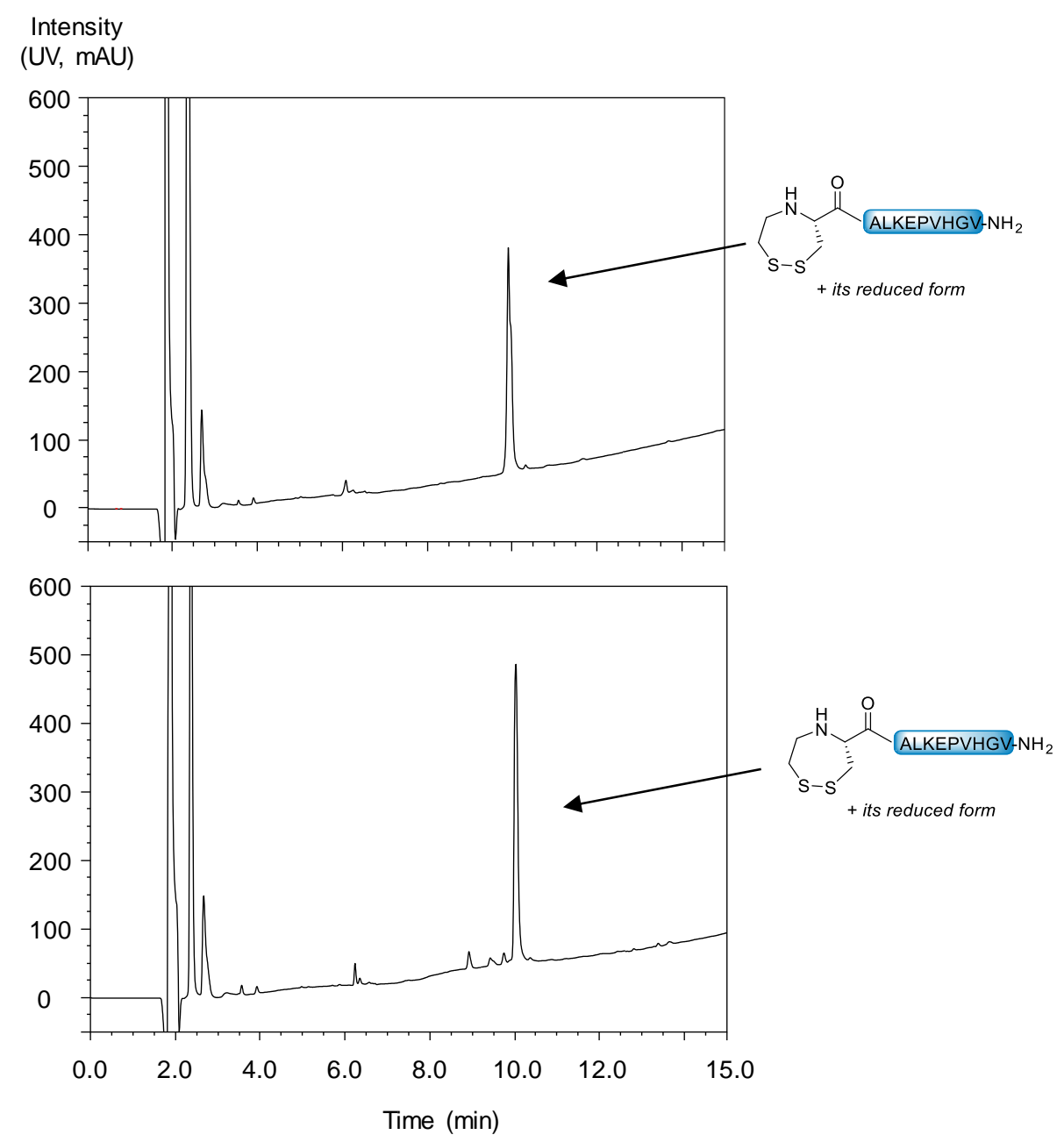

Figure S 87. Stability of the SutCys peptide in the presence of MPAA + TCEP $(\mathrm{pH}=6.06)$. LC trace: eluent A $0.10 \%$ TFA in water, eluent B $0.10 \%$ TFA in $\mathrm{CH}_{3} \mathrm{CN} /$ water: $4 / 1$ by vol., C18 column, gradient $0-50 \%$ B in $15 \mathrm{~min}, 30{ }^{\circ} \mathrm{C}, 1 \mathrm{~mL} / \mathrm{min}$, UV detection at $215 \mathrm{~nm}$.

\subsection{NCL under low reducing conditions}

When SutCys-ALKEPVHGV-NH2 peptide and peptidyl thioester 4 were reacted under mild reducing conditions (MPAA), the resulting peptide featuring the SutCys unit in the form of a cyclic disulfide at the ligation junction was recovered as the only product of the ligation with $53 \%$ yield (Figure $\mathrm{S} 88$ ). 


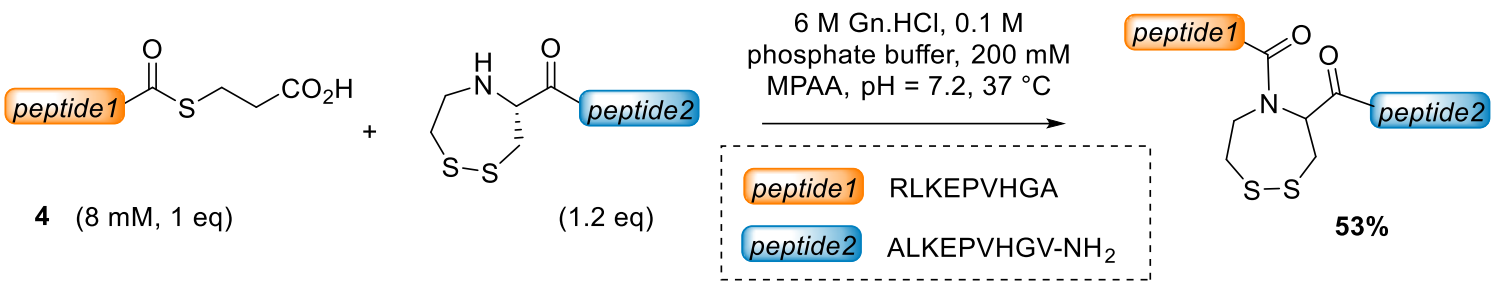

Figure S 88. Ligation reaction of SutCys-ALKEPVHGV-NH2 peptide with peptidyl MPAthioester $\mathbf{4}$ under low reducing conditions.

\section{Protocol}

To a solution of $\mathrm{Gn} \cdot \mathrm{HCl}(1.03 \mathrm{~g})$ in $0.1 \mathrm{M}$, pH 7.4 phosphate buffer $(1.08 \mathrm{~mL})$ was added MPAA $(60.6 \mathrm{mg})$ and the $\mathrm{pH}$ of the mixture was adjusted to 7.21 by addition of $6 \mathrm{M} \mathrm{NaOH}$. SutCys-ALKEPVHGV-NH2 peptide (22.4 mg, $15.4 \mu \mathrm{mol}, 1.2 \mathrm{eq})$ and RLKEPVHGA-MPA peptide 4 (20.0 mg, $12.9 \mu \mathrm{mol}, 1 \mathrm{eq})$ were then successively dissolved in this solution (1.61 $\mathrm{mL}$ ) and the reaction mixture was stirred at $37^{\circ} \mathrm{C}$ for $24 \mathrm{~h}$. The mixture was then acidified with $10 \% \mathrm{AcOH}$ in water $(23 \mathrm{~mL})$ and extracted with $\mathrm{Et}_{2} \mathrm{O}(5 \times)$ to remove the MPAA. Purification of the crude by HPLC (eluent A: water containing $0.1 \%$ TFA, eluent B: acetonitrile in water $4 / 1$ containing $0.1 \%$ TFA, $50^{\circ} \mathrm{C}$, detection at $215 \mathrm{~nm}, 6 \mathrm{~mL} / \mathrm{min}, 0-10 \%$ eluent $\mathrm{B}$ in $5 \mathrm{~min}$, then 10-35\% eluent B in 35 min, C18XBridge column) provided RLKEPVHGA-SutCysALKEPVHGV-NH2 peptide as a white solid after lyophilisation (19.2 mg, 53\%). 
a) $\mathrm{t}=20 \mathrm{~s}$

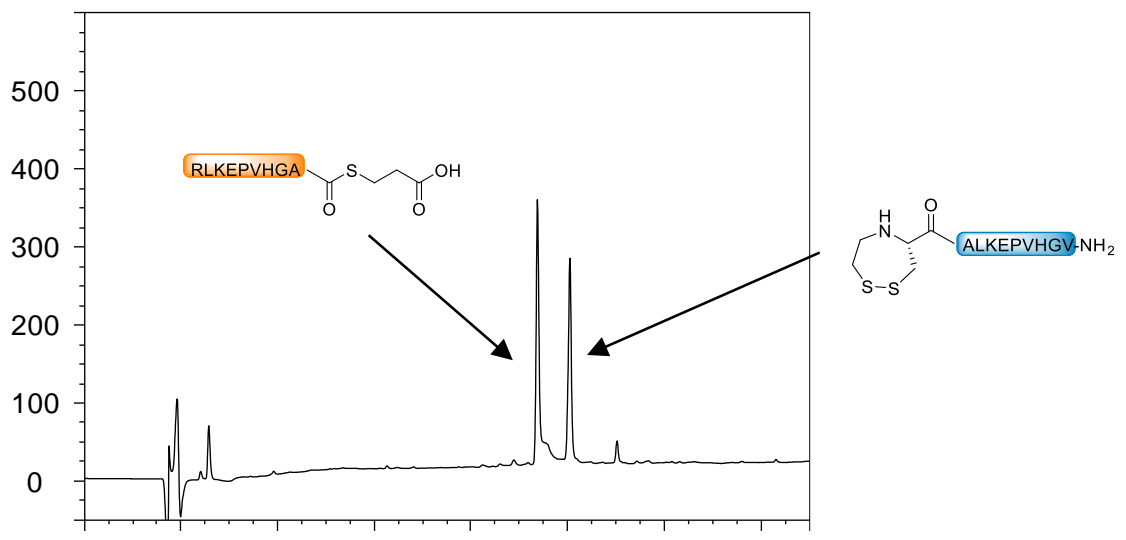

b) $\mathrm{t}=5.3 \mathrm{~h}$

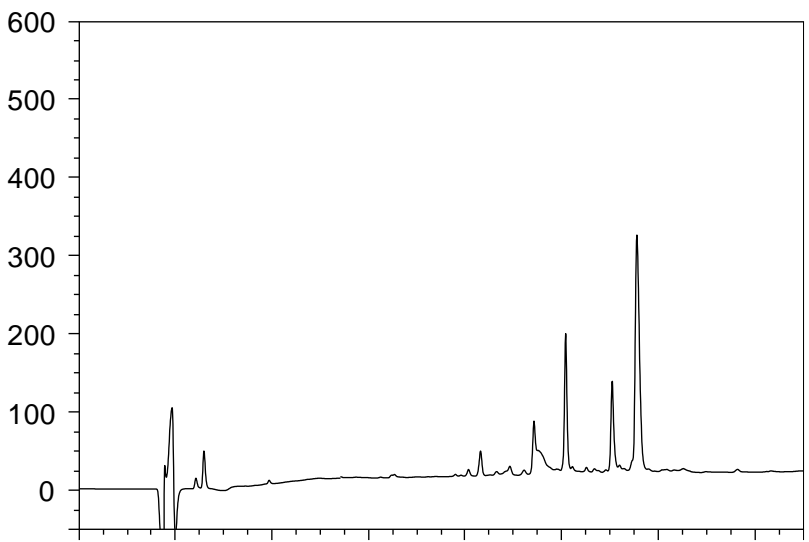

b) $\mathrm{t}=22 \mathrm{~h}$

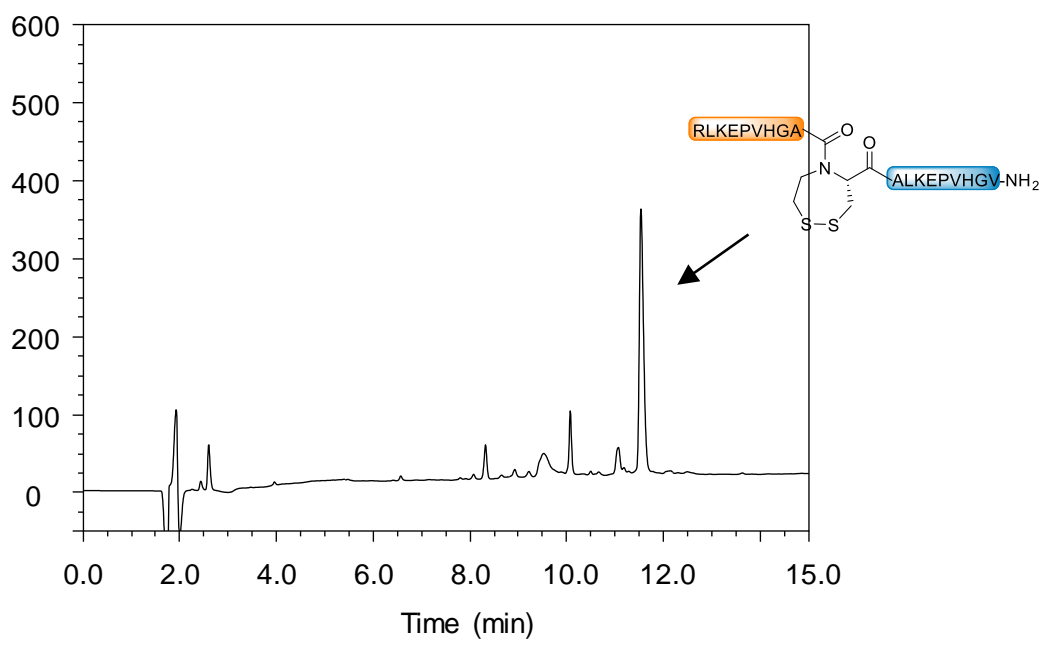

Figure S 89. Monitoring of the ligation between SutCys peptide SutCysALKEPVHGV-NH and peptidyl MPA thioester RLKEPVHGA-MPA. LC trace: eluent A 0.10\% TFA in water, eluent $\mathrm{B} 0.10 \%$ TFA in $\mathrm{CH}_{3} \mathrm{CN} /$ water: $4 / 1$ by vol., $\mathrm{C} 18$ column, gradient $0-50 \%$ B in 15 min, $30{ }^{\circ} \mathrm{C}, 1 \mathrm{~mL} / \mathrm{min}$, UV detection at $215 \mathrm{~nm}$. 
Characterization of RLKEPVHGA-SutCys-ALKEPVHGV-NH $\mathrm{H}_{2}$ peptide:

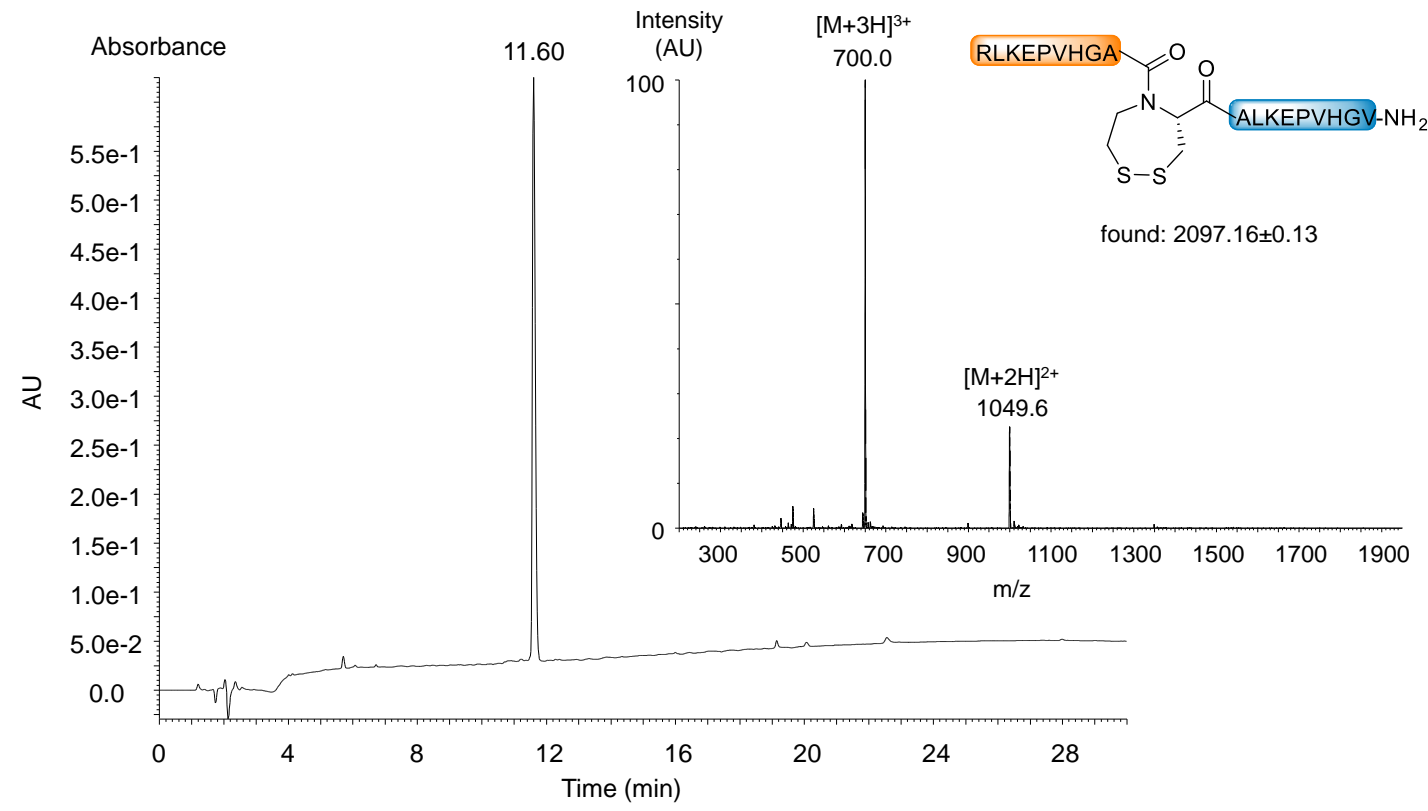

Figure S 90. LC-MS analysis of RLKEPVHGA-SutCys-ALKEPVHGV-NH2 peptide. LC trace: eluent A $0.10 \%$ TFA in water, eluent B $0.10 \%$ TFA in $\mathrm{CH}_{3} \mathrm{CN} /$ water: $4 / 1$ by vol. $\mathrm{C} 18$ Xbridge BEH $300 \AA 5 \mu \mathrm{m}(4.6 \times 250 \mathrm{~mm})$ column, gradient $0-100 \% \mathrm{~B}$ in $30 \mathrm{~min}, 50{ }^{\circ} \mathrm{C}, 1$ $\mathrm{mL} / \mathrm{min}$, UV detection $(215 \mathrm{~nm})$. MS trace: $1049.6\left([\mathrm{M}+2 \mathrm{H}]^{2+}\right), 700.0\left([\mathrm{M}+3 \mathrm{H}]^{3+}\right)$; calcd. for M (average): 2097.54, found: 2097.2.

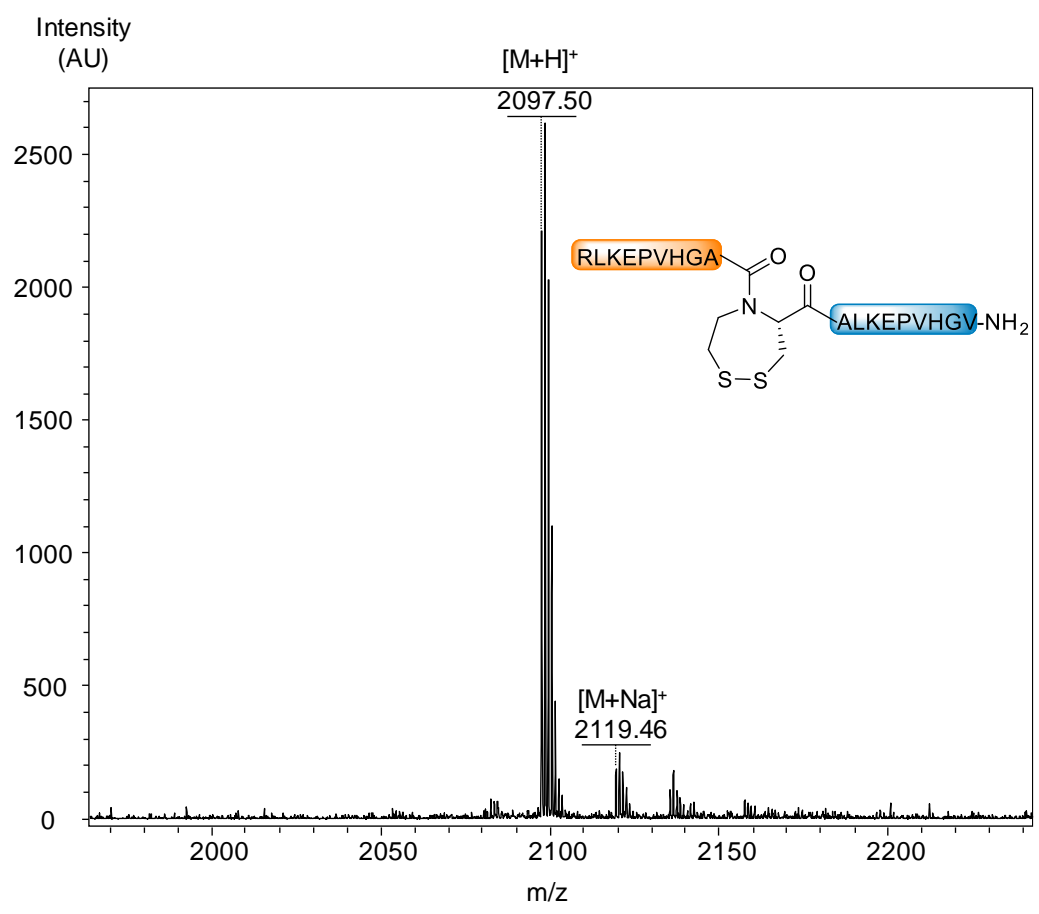

Figure S 91. MALDI-TOF analysis of RLKEPVHGA-SutCys-ALKEPVHGV-NH2 peptide. Matrix $\alpha$-cyano-4-hydroxycinnamic acid, positive detection mode, calcd. for $[\mathrm{M}+\mathrm{H}]^{+}$ (monoisotopic): 2097.11, found: 2097.5. 


\subsection{NCL under strong reducing conditions}
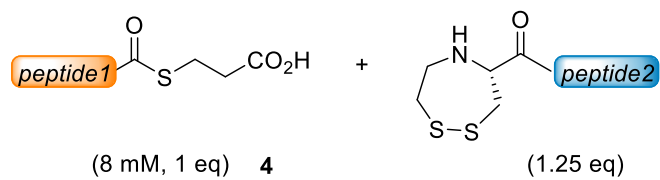

peptide

peptide2.
RLKEPVHGA

ALKEPVHGV-NH

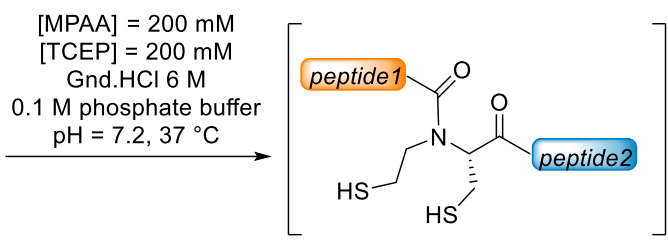

1) MPAA extraction 2) $I_{2}$ in DMSO

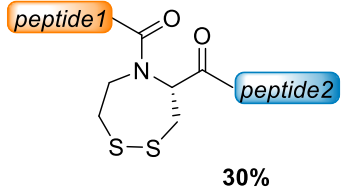

Figure S 92: Ligation reaction of SutCys-ALKEPVHGV-NH2 peptide with peptidyl MPAthioester 4 under strong reducing conditions.

\section{Protocol}

To a solution of $\mathrm{Gn} \cdot \mathrm{HCl}(573 \mathrm{mg})$ in $0.1 \mathrm{M}$ phosphate buffer $(0.6 \mathrm{~mL})$ were successively added TCEP $(53.7 \mathrm{mg}$ ) and MPAA (33.6 $\mathrm{mg}$ ) and the $\mathrm{pH}$ of the mixture was adjusted to 7.2 by addition of $6 \mathrm{M} \mathrm{NaOH}$. SutCys-ALKEPVHGV-NH2 $(8.00 \mathrm{mg}, 5.51 \mu \mathrm{mol}, 1.25 \mathrm{eq})$ and peptidyl thioester 4 (6.83 mg, $4.40 \mu \mathrm{mol}, 1 \mathrm{eq})$ were then successively dissolved in this solution $(0.55$ $\mathrm{mL}$ ) and the reaction mixture was maintained at $37^{\circ} \mathrm{C}$.

After $48 \mathrm{~h}$ reaction, a $2 \mu \mathrm{L}$ aliquot was taken from the reaction mixture and quenched by adding $10 \% \mathrm{AcOH}$ in water $(100 \mu \mathrm{L})$. The sample was then extracted with $\mathrm{Et}_{2} \mathrm{O}$ to remove MPAA prior to LC-MS analysis (Figure S 93). The ligation product $\left(t_{R}=11.48 \mathrm{~min}\right)$ featuring the SutCys unit in its reduced, ring-opened form was detected in the crude, in mixture with the unreacted SutCys-ALKEPVHGV-NH2 peptide $\left(t_{R}=10.10 \mathrm{~min}\right)$ and with the peptide resulting from the hydrolysis of the starting peptide thioester $\left(t_{R}=8.37 \mathrm{~min}\right)$.

The ligation product was isolated in the form of the cyclic disulfide after oxidation with iodine. Experimentally, the ligation mixture was acidified with $10 \% \mathrm{AcOH}$ in water $(3.5 \mathrm{~mL})$ and extracted with $\mathrm{Et}_{2} \mathrm{O}(5 \times)$ to remove the MPAA. A solution of iodine $(158 \mathrm{mg})$ in DMSO (2 $\mathrm{mL}$ ) was then added dropwise until persistency of the yellow color of iodine. The excess of iodine was then quenched with a solution of DTT ( $40 \mathrm{mg})$ in $20 \% \mathrm{AcOH}(4 \mathrm{~mL})$ and the mixture was diluted with water (final volume: $10 \mathrm{~mL}$ ). Purification of the crude by HPLC (eluent A: water containing $0.1 \% \mathrm{TFA}$, eluent $\mathrm{B}$ : acetonitrile in water $4 / 1$ containing $0.1 \% \mathrm{TFA}, 50^{\circ} \mathrm{C}$, detection at $215 \mathrm{~nm}, 6 \mathrm{~mL} / \mathrm{min}, 0-10 \%$ eluent $\mathrm{B}$ in $5 \mathrm{~min}$, then 10-30\% eluent $\mathrm{B}$ in $30 \mathrm{~min}$, C18XBridge column) provided RLKEPVHGA-SutCys-ALKEPVHGV-NH2 as a white solide after lyophilisation (3.70 mg, 30\%). 


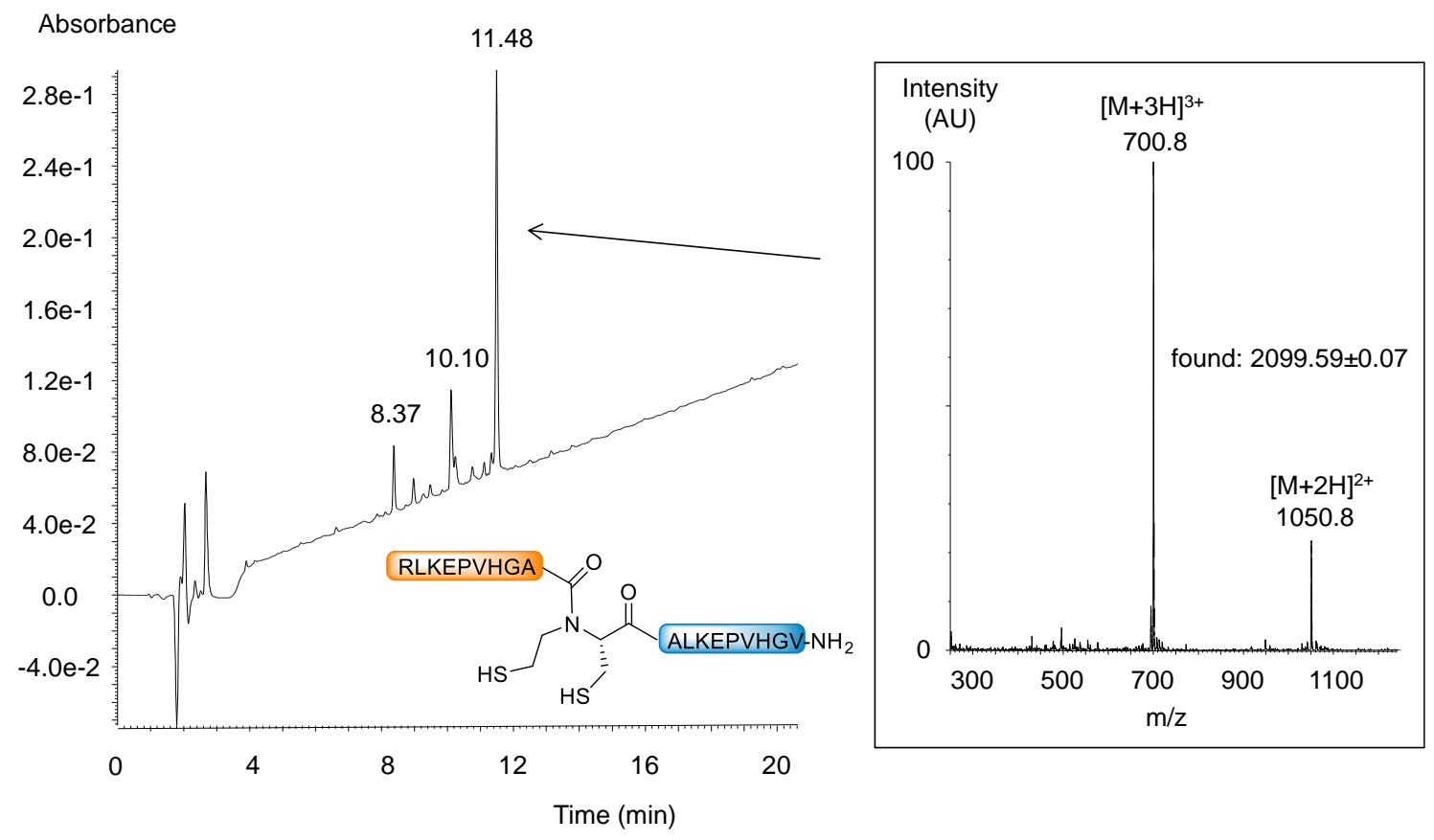

Figure S 93. LC-MS analysis of the ligation mixture. LC trace: eluent A $0.10 \%$ TFA in water, eluent B $0.10 \%$ TFA in $\mathrm{CH}_{3} \mathrm{CN} /$ water: $4 / 1$ by vol. $\mathrm{C} 18$ Xbridge $\mathrm{BEH} 300 \AA 5 \mu \mathrm{m}(4.6 \times 250$ $\mathrm{mm}$ ) column, gradient $0-100 \% \mathrm{~B}$ in $30 \mathrm{~min}, 50{ }^{\circ} \mathrm{C}, 1 \mathrm{~mL} / \mathrm{min}$, UV detection $(215 \mathrm{~nm})$. MS trace for SutCys ligated peptide $\left(\mathrm{t}_{\mathrm{R}}=11.48 \mathrm{~min}\right): 1050.8\left([\mathrm{M}+2 \mathrm{H}]^{2+}\right), 700.8\left([\mathrm{M}+3 \mathrm{H}]^{3+}\right)$; calcd. for M (average): 2099.55, found: 2099.6. 


\section{Mechanistic studies (Figure 3)}

\subsection{Effect of pH (Figure 3b)}

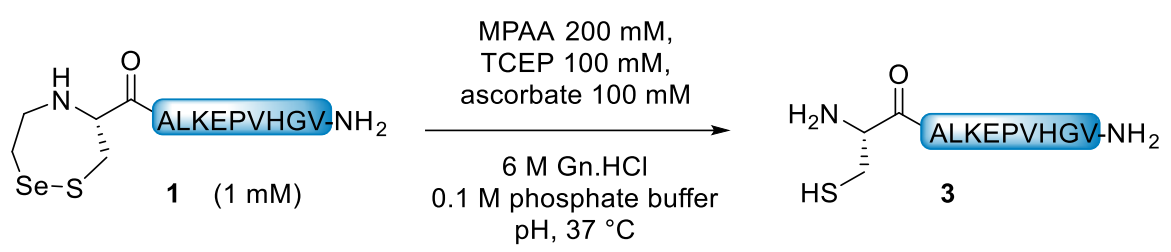

General procedure illustrated with the following conditions:

$1 \mathrm{mM}$ peptide, $200 \mathrm{mM}$ MPAA, $100 \mathrm{mM}$ TCEP, $100 \mathrm{mM}$ ascorbate, $\mathrm{pH} 6.9$

in $0.1 \mathrm{M}$ phosphate buffer

To a solution of $\mathrm{Gn} \cdot \mathrm{HCl}(287 \mathrm{mg})$ in $0.1 \mathrm{M}, \mathrm{pH} 7.4$ phosphate buffer $(300 \mu \mathrm{L})$ were added TCEP.HCl (14.3 mg), sodium ascorbate $(9.9 \mathrm{mg})$ and MPAA $(16.8 \mathrm{mg})$ and the $\mathrm{pH}$ of the mixture was adjusted to 6.90 by addition of $6 \mathrm{M} \mathrm{NaOH}$. SetCys peptide $1(0.48 \mathrm{mg}, 0.32 \mu \mathrm{mol})$ was then dissolved in the buffered solution $(320 \mu \mathrm{L})$ and the reaction mixture was stirred at 37 ${ }^{\circ} \mathrm{C}$. The progress of the reaction was monitored by HPLC. For each point, an $8 \mu \mathrm{L}$ aliquot was taken from the reaction mixture and quenched by adding $10 \% \mathrm{AcOH}$ in water $(100 \mu \mathrm{L})$. The sample was then extracted with $\mathrm{Et}_{2} \mathrm{O}$ to remove MPAA prior to HPLC analysis. The kinetic data were then fitted to a pseudo-first order kinetic law using KinteK Explorer Software ${ }^{T M}$ (Figure S 94).

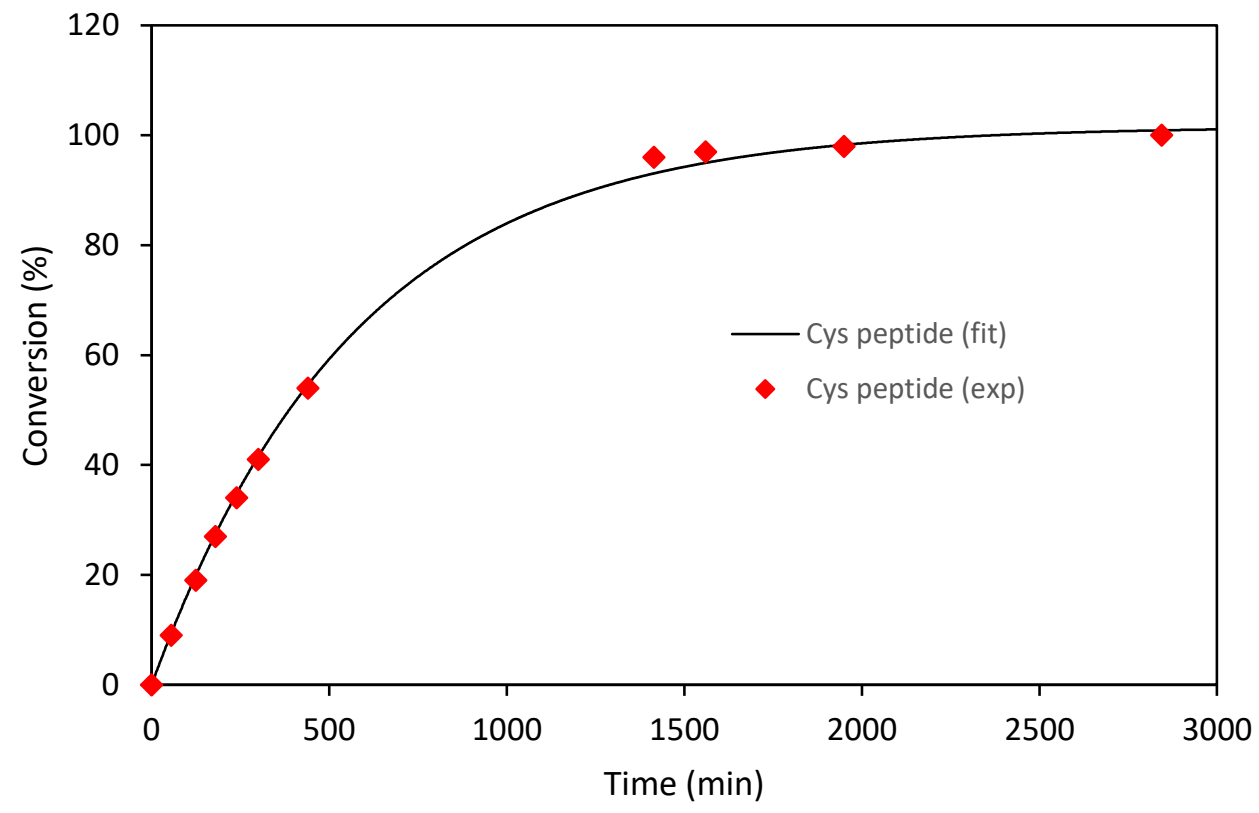

Figure S 94. Fitting of the reaction leading to a loss of the $N$-selenoethyl appendage on SetCys peptides at $\mathrm{pH} 6.90$ using KinteK Explorer Software ${ }^{T M}$ (Version 7.2.180216., Kintek Corporation - https://kintekcorp.com/software/). Red diamonds correspond to experimental values / Gray line corresponds to fitted data. 
The reaction leading to a loss of the $N$-selenoethyl appendage on SetCys peptides was repeated at various $\mathrm{pH}(4.99<\mathrm{pH}<8.49)$. Corresponding rate constants are given in Table $\mathrm{S} 3$.

Table S 3. Effect of the $\mathrm{pH}$ on the conversion of SetCys-ALKEPVHGV-NH 1 into CALKEPVHGV-NH2 3. Rate constants $\mathrm{k}_{1}$ were extracted using KinteK Explorer Software ${ }^{T M}$.

\begin{tabular}{ccc}
$\mathbf{p H}$ & $\mathbf{k} \mathbf{1}\left(\mathbf{m i n}^{-\mathbf{1}}\right)$ & standard error \\
\hline 4.99 & 0.00137 & 0.00083 \\
5.25 & 0.00191 & 0.00074 \\
5.54 & 0.00203 & 0.00061 \\
5.98 & 0.00253 & 0.00043 \\
6.52 & 0.00202 & 0.00126 \\
6.90 & 0.00174 & 0.00024 \\
7.22 & 0.00119 & 0.00080 \\
7.55 & 0.00089 & 0.00003 \\
8.03 & 0.00047 & 0.00005 \\
8.49 & 0.00014 & 0.00003
\end{tabular}

\subsection{Effect of Cys thiol: control experiment with a SetAla peptide (Figure 3d)}

\subsubsection{Decomposition of SetAla peptide into Ala peptide under strong reducing conditions}

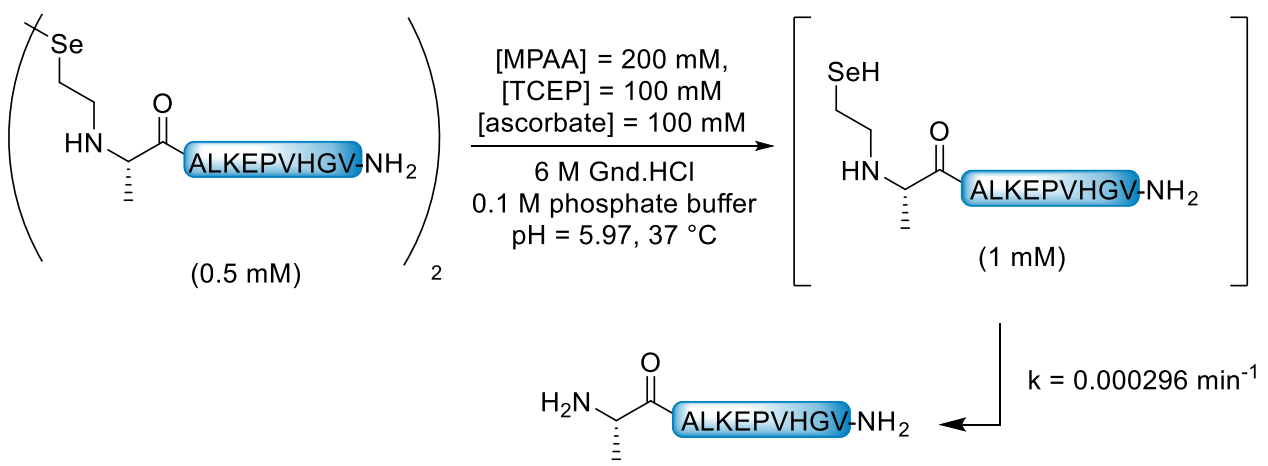

Figure S 95. Decomposition of SetAla peptide into an Ala peptide.

To a solution of $\mathrm{Gn} \cdot \mathrm{HCl}(287 \mathrm{mg})$ in $0.1 \mathrm{M}$, pH 7.4 phosphate buffer $(300 \mu \mathrm{L})$ were added TCEP.HCl $(14.3 \mathrm{mg})$, sodium ascorbate $(9.9 \mathrm{mg})$ and MPAA $(16.8 \mathrm{mg})$ and the $\mathrm{pH}$ of the mixture was adjusted to 5.97 by addition of $6 \mathrm{M} \mathrm{NaOH}$. SetAla peptide $(0.47 \mathrm{mg}, 0.16 \mu \mathrm{mol})$ was then dissolved in the buffered solution $(320 \mu \mathrm{L})$ and the reaction mixture was stirred at 37 ${ }^{\circ} \mathrm{C}$. The progress of the reaction was monitored by HPLC (Figure S 96). For each point, an $8 \mu \mathrm{L}$ aliquot was taken from the reaction mixture and quenched by adding $10 \% \mathrm{AcOH}$ in water (100 $\mu \mathrm{L})$. The sample was then extracted with $\mathrm{Et}_{2} \mathrm{O}$ to remove MPAA prior to HPLC analysis. The 
kinetic data were then fitted to a pseudo-first order kinetic law using KinteK Explorer Software $^{T M}$ (Figure S 97).

Intensity

(UV, mAU)

a) $\mathrm{t}=1.5 \mathrm{~h}$

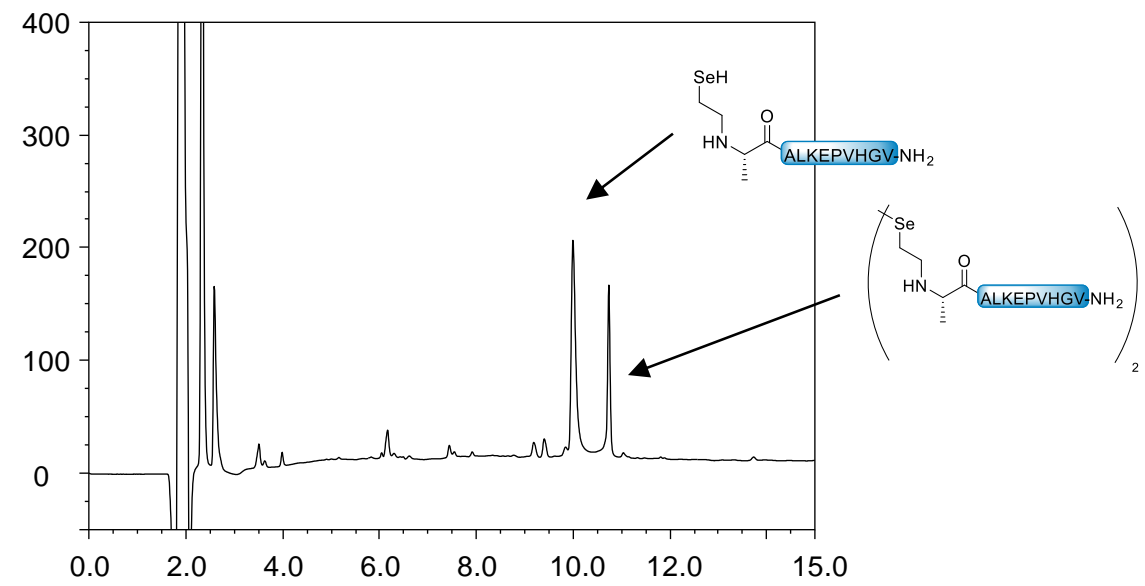

b) $t=22 h$

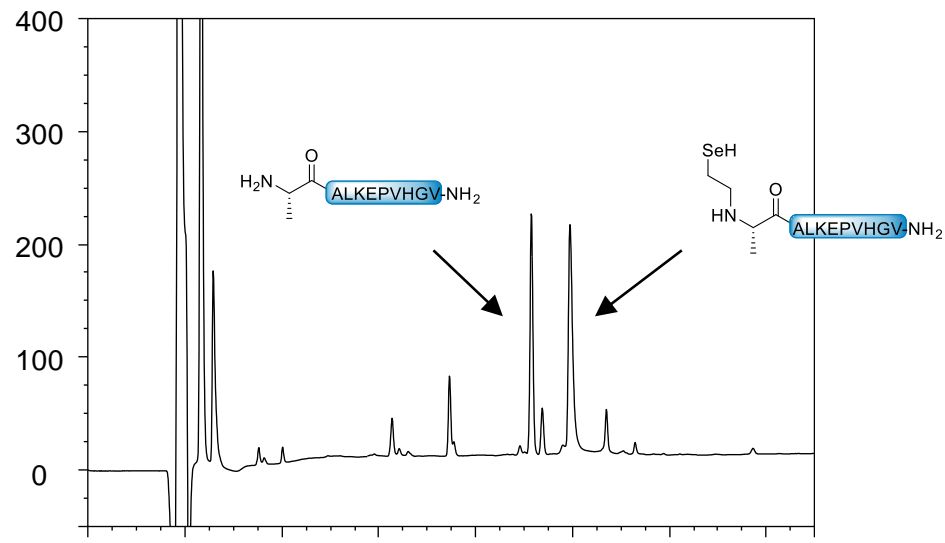

b) $t=142 h$

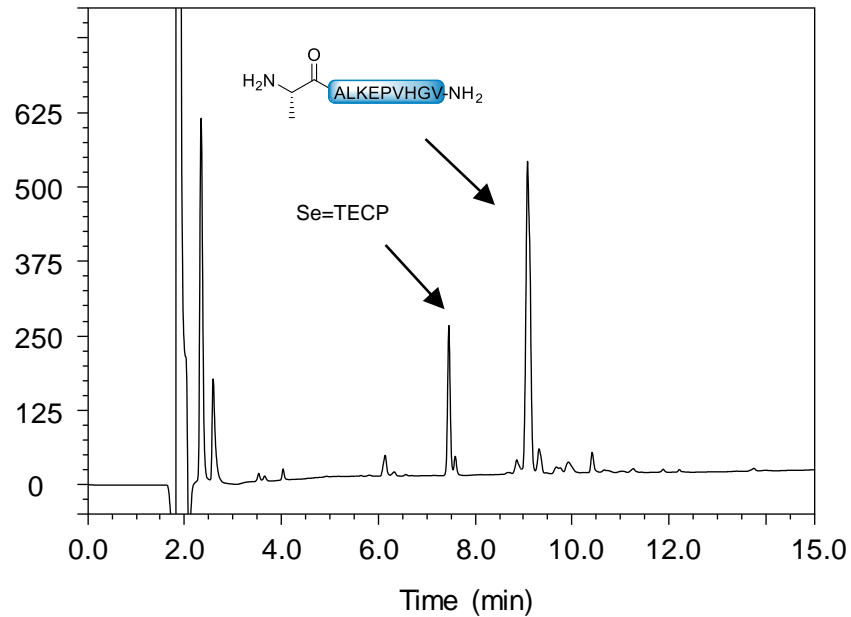

Figure S 96. Monitoring of the conversion of SetAla peptide 1 into Ala peptide using MPAA + TCEP. LC trace: eluent A $0.10 \%$ TFA in water, eluent B $0.10 \%$ TFA in $\mathrm{CH}_{3} \mathrm{CN}, \mathrm{C} 18$ column, gradient $0-50 \% \mathrm{~B}$ in $15 \mathrm{~min}, 30{ }^{\circ} \mathrm{C}, 1 \mathrm{~mL} / \mathrm{min}$, UV detection at $215 \mathrm{~nm}$. 


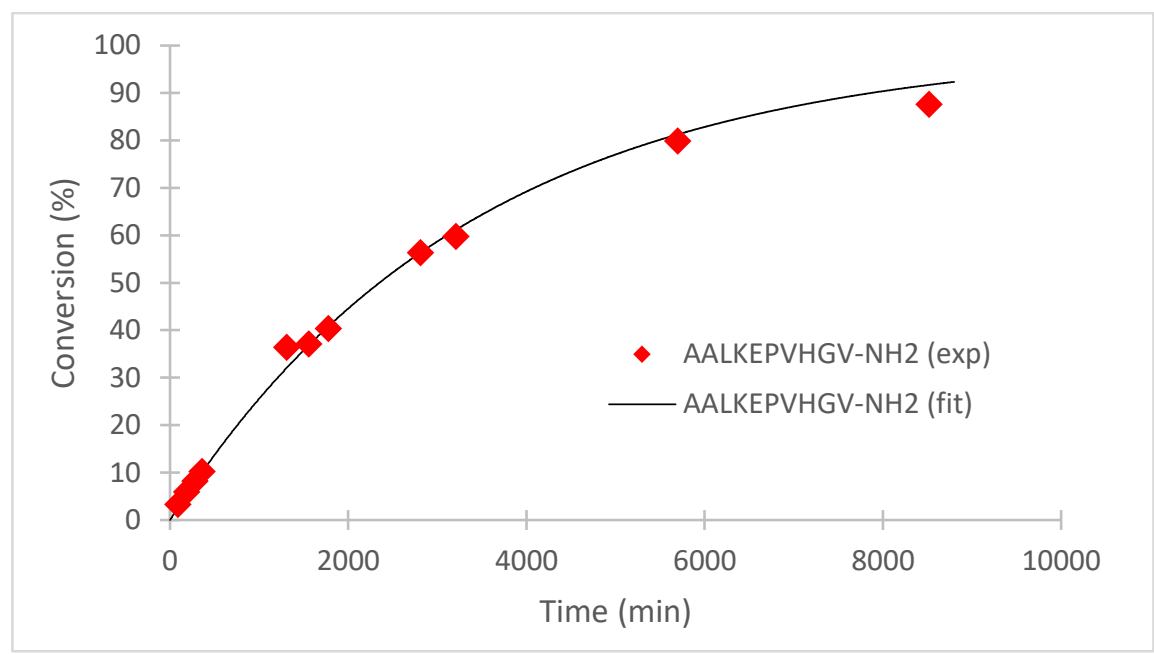

Figure S 97. Fitting of the reaction leading to a loss of the $N$-selenoethyl appendage on SetAla peptides at $\mathrm{pH} 5.97$ using KinteK Explorer Software ${ }^{T M}$ (Version 7.2.180216., Kintek Corporation - https://kintekcorp.com/software/). $\mathrm{k}=0.000296 \pm 0.000091$. Red diamonds correspond to experimental values / Black line corresponds to fitted data.

The AALKEPVHGV-NH2 peptide produced by decomposition of SetAla peptide during the reaction was purified by HPLC and its sequence was confirmed by MS-MS analysis (Figure $\mathrm{S}$ 98 and Figure S 99).

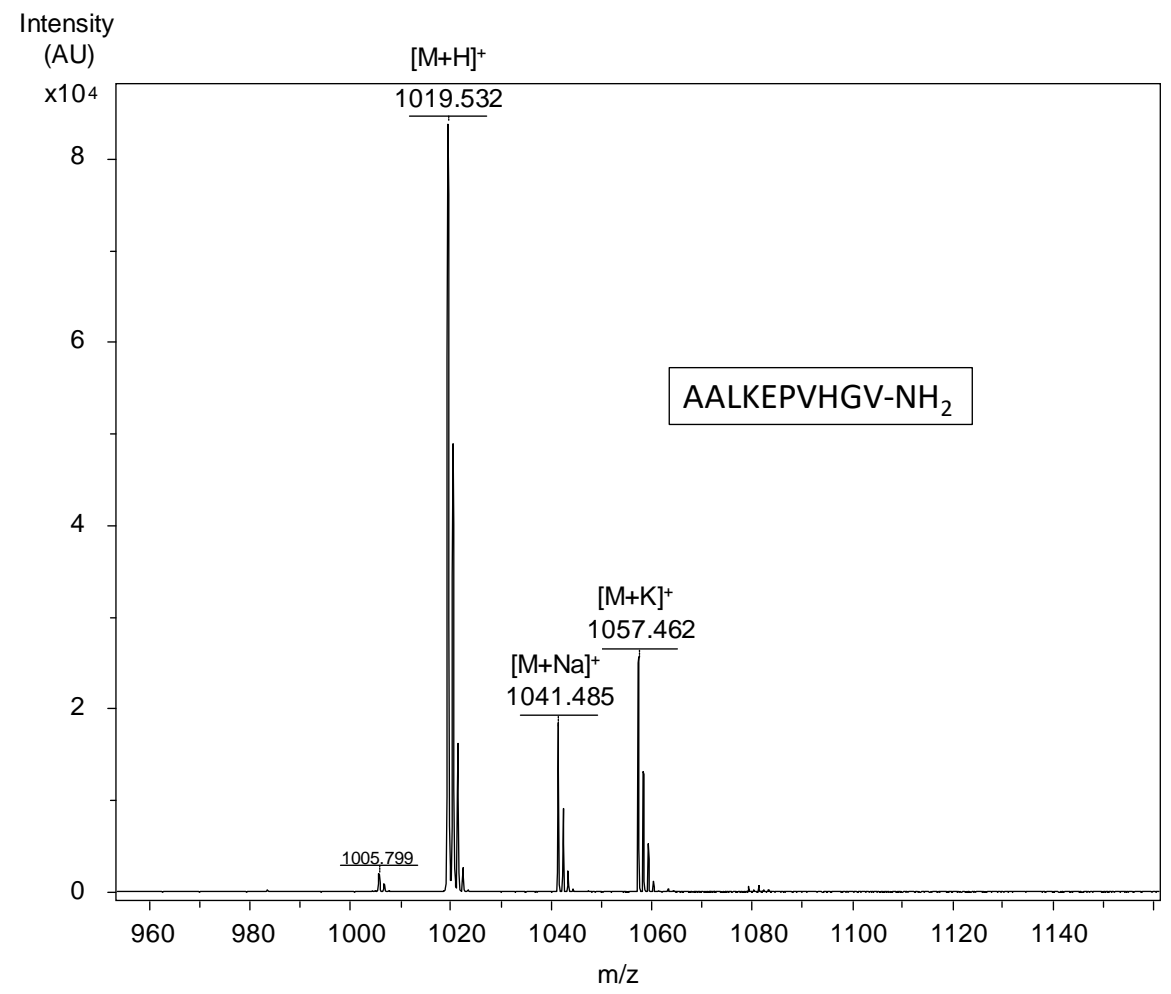

Figure S 98. MALDI-TOF analysis of AALKEPVHGV-NH2 peptide. Matrix $\alpha$-cyano-4hydroxycinnamic acid, positive detection mode, calcd. for $[\mathrm{M}+\mathrm{H}]^{+}$(monoisotopic): 1019.60, found: 1019.53 . 


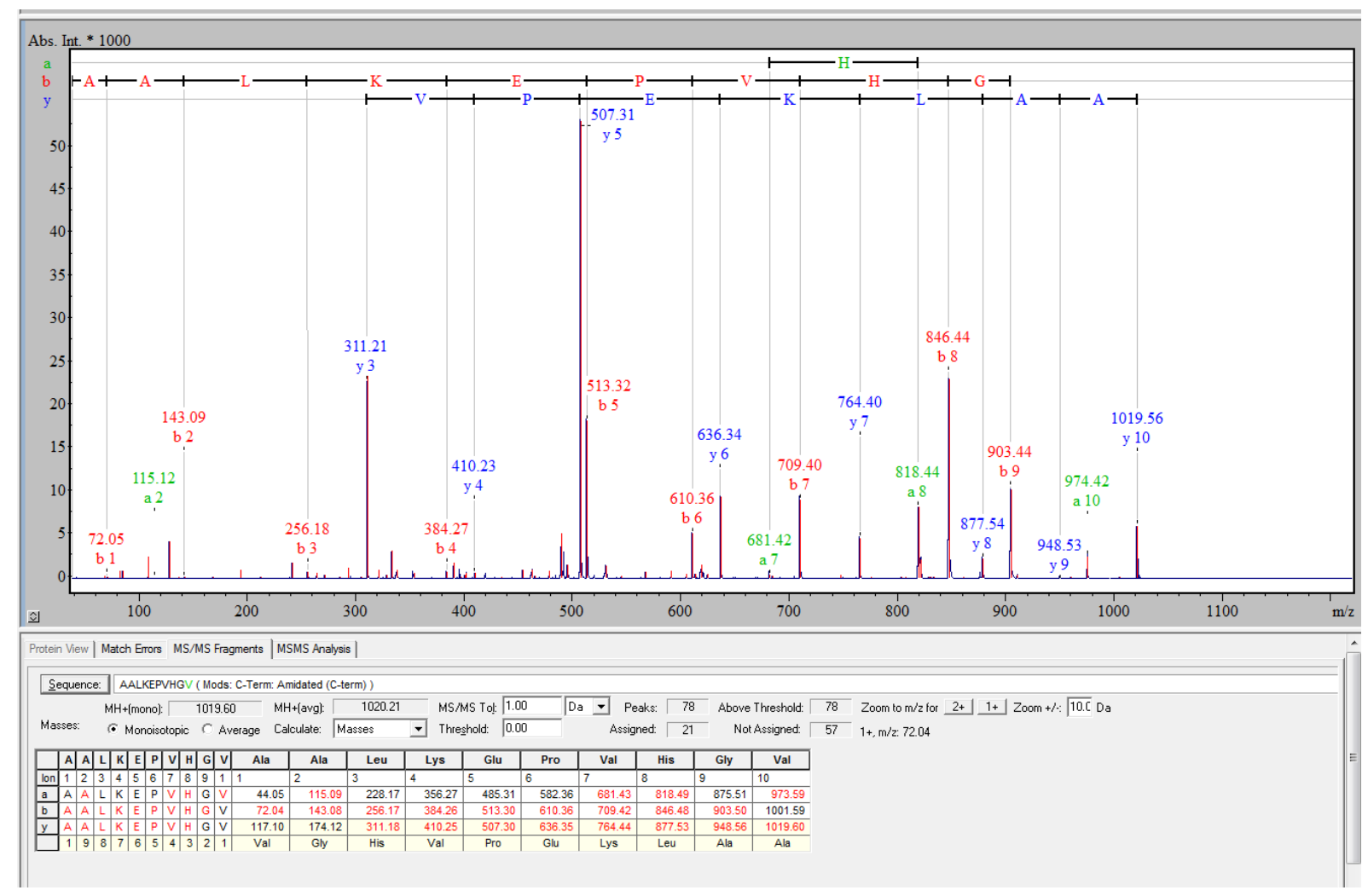

Figure S 99. MALDI-TOF-TOF sequencing of AALKEPVHGV-NH2 peptide. Matrix $\alpha$ cyano-4-hydroxycinnamic acid, positive detection mode. The MS-MS analysis confirms that the SetAla residue is converted into the Ala amino acid in the peptide.

\subsubsection{Ligation of SetAla peptide under strong reducing conditions}

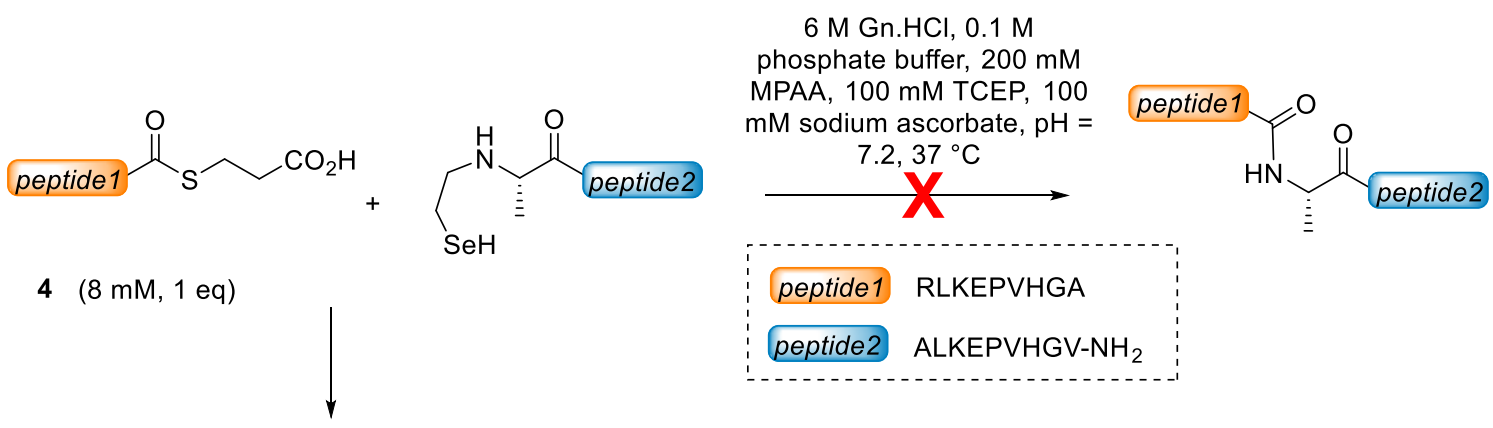

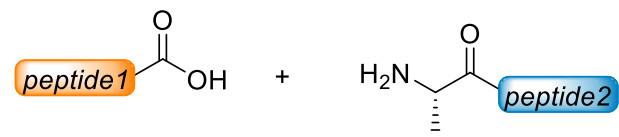

Figure S 100. Ligation reaction of SetAla peptide with peptidyl MPA-thioester 4. 


\section{Protocol}

To $500 \mu \mathrm{L}$ of a $6 \mathrm{M}$ solution of $\mathrm{Gn} \cdot \mathrm{HCl}$ in $0.1 \mathrm{M} \mathrm{pH} 7.4$ phosphate buffer were successively added MPAA $(16.8 \mathrm{mg})$, TCEP $(14.3 \mathrm{mg})$ and sodium ascorbate $(9.9 \mathrm{mg})$. The $\mathrm{pH}$ of the mixture was adjusted to 7.28 by addition of $6 \mathrm{M} \mathrm{NaOH}(61,5 \mu \mathrm{L})$. In $140 \mu \mathrm{L}$ of the previous solution were dissolved SetAla-ALKEPVHGV-NH2 peptide $(1.97 \mathrm{mg}, 1.2 \mathrm{eq})$ and RLKEPVHGA-MPA thioester $(1.73 \mathrm{mg}, 1 \mathrm{eq})$. A pH of 6.90 was measured after addition of the peptide segments. The reaction mixture was stirred at $37^{\circ} \mathrm{C}$ and monitored by HPLC (Figure S 101). For each point, a $2 \mu \mathrm{L}$ aliquot was taken from the reaction mixture and quenched by adding $10 \% \mathrm{AcOH}$ in water $(100 \mu \mathrm{L})$. The sample was then extracted with $\mathrm{Et}_{2} \mathrm{O}$ to remove MPAA prior to HPLC analysis.

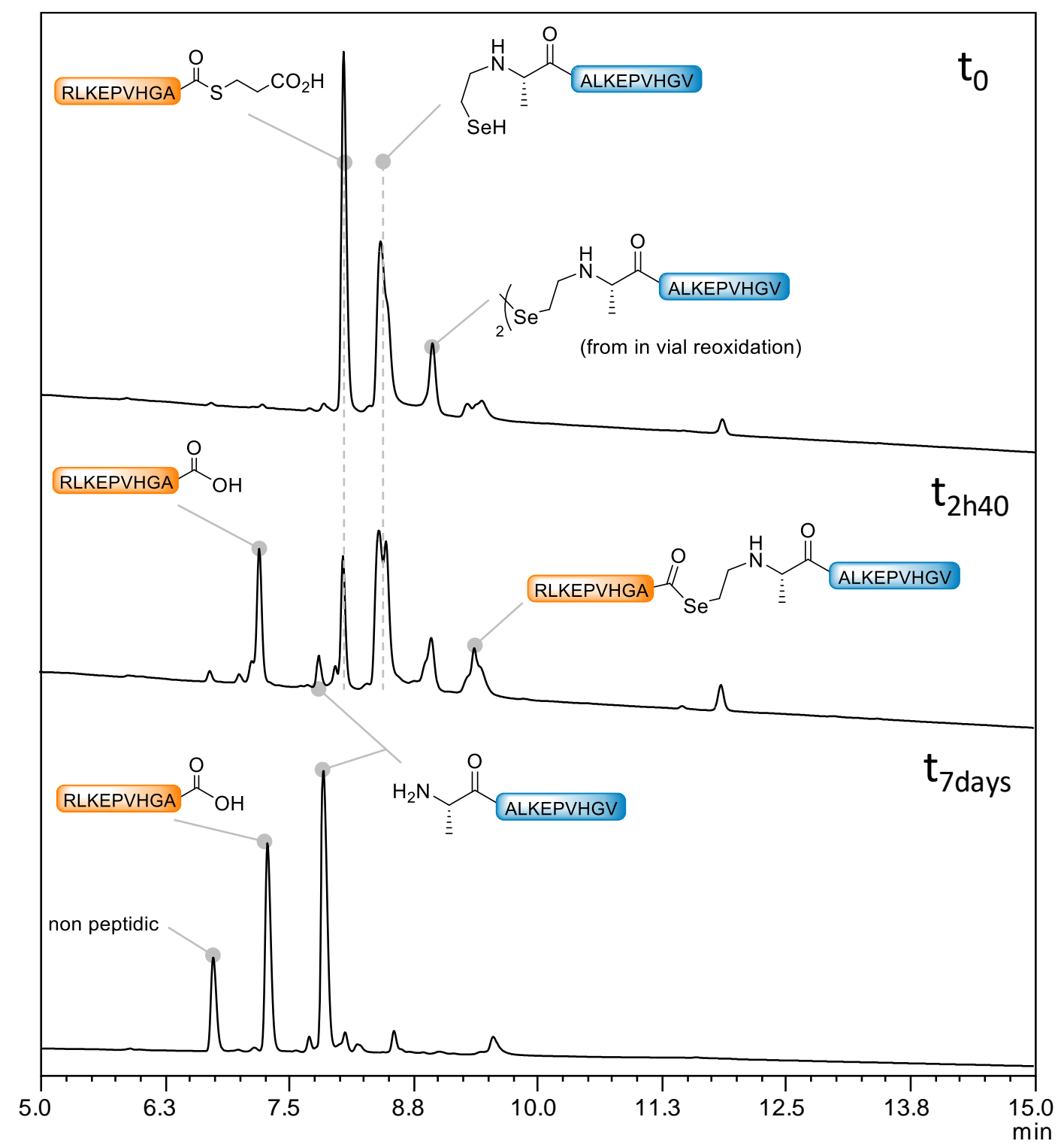

Figure S 101. Monitoring of the reaction between a SetAla peptide and a peptidyl MPAthioester in the presence of MPAA, TCEP and sodium ascorbate. LC trace: eluent A $0.10 \%$ TFA in water, eluent $\mathrm{B} 0.10 \%$ TFA in $\mathrm{CH}_{3} \mathrm{CN}, \mathrm{C} 18$ column, gradient $0-50 \% \mathrm{~B}$ in $15 \mathrm{~min}, 30^{\circ} \mathrm{C}$, $1 \mathrm{~mL} / \mathrm{min}$, UV detection at $215 \mathrm{~nm}$. 


\section{Kinetic model of SetCys-mediated NCL under strong reducing conditions (Figure 4)}

SetCys peptide 1 and peptidyl thioester $\mathbf{4}$ can be assembled in the presence of TCEP into ligated product 6 having a cysteine as a junction residue. Such a reaction implies that the $N$-selenoethyl appendage of the SetCys unit is lost during the process.

A kinetic study of the ligation shows also the transient accumulation of species $\mathbf{5}$ at the beginning of the reaction (Figure S 102).

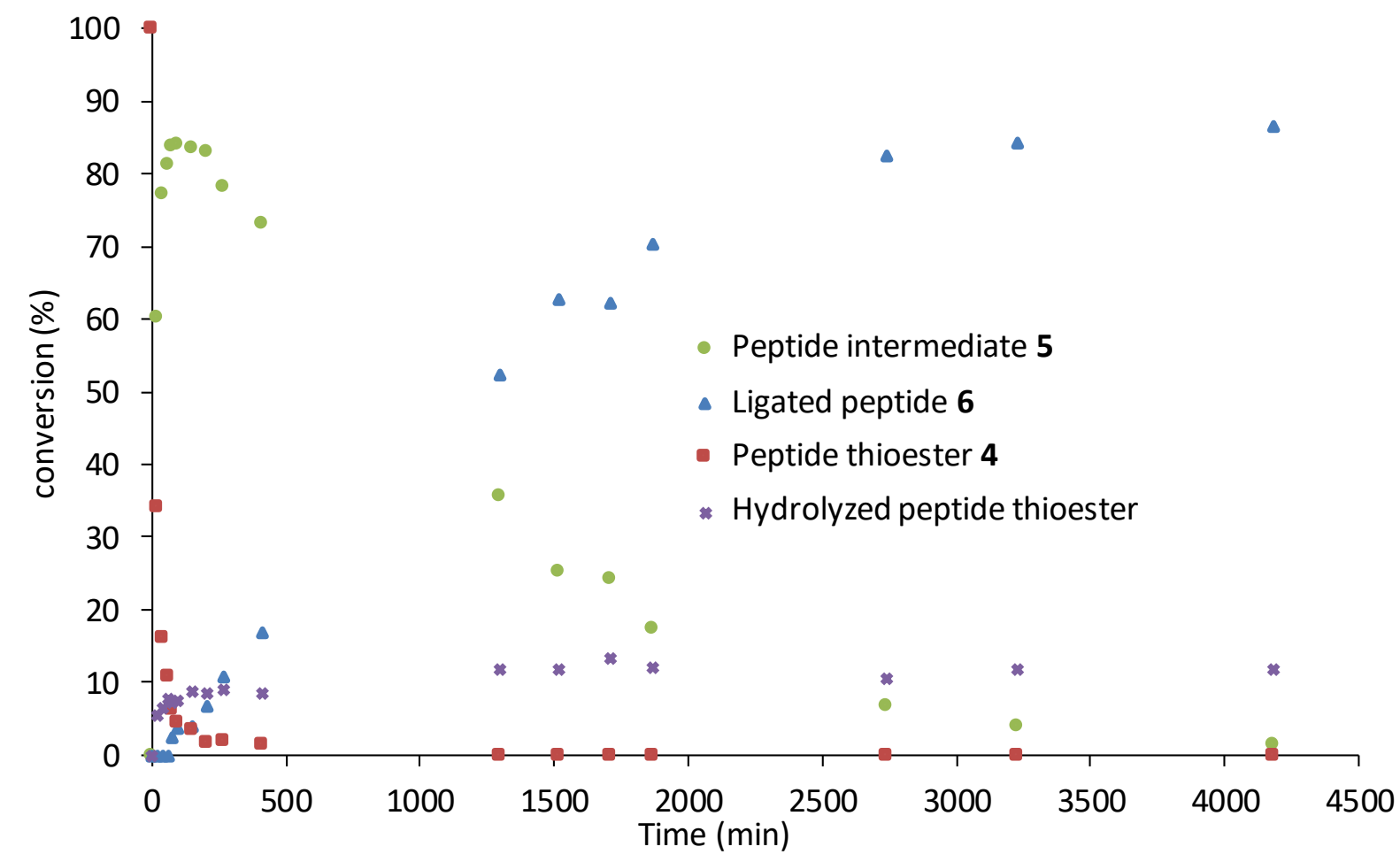

Figure S 102. HPLC monitoring of the reaction between SetCys peptide $\mathbf{1}$ and peptide thioester 4 performed in the presence of TCEP. For each point, a $2 \mu \mathrm{L}$ aliquot was taken from the reaction mixture and quenched by adding $10 \% \mathrm{AcOH}$ in water $(100 \mu \mathrm{L})$. The sample was then extracted with $\mathrm{Et}_{2} \mathrm{O}$ to remove MPAA prior to HPLC analysis. The proportion of each species in the mixture was determined by HPLC using UV detection at $215 \mathrm{~nm}$.

On the basis of the preceding observations, we have proposed the following mechanistic model (Figure S 103). 


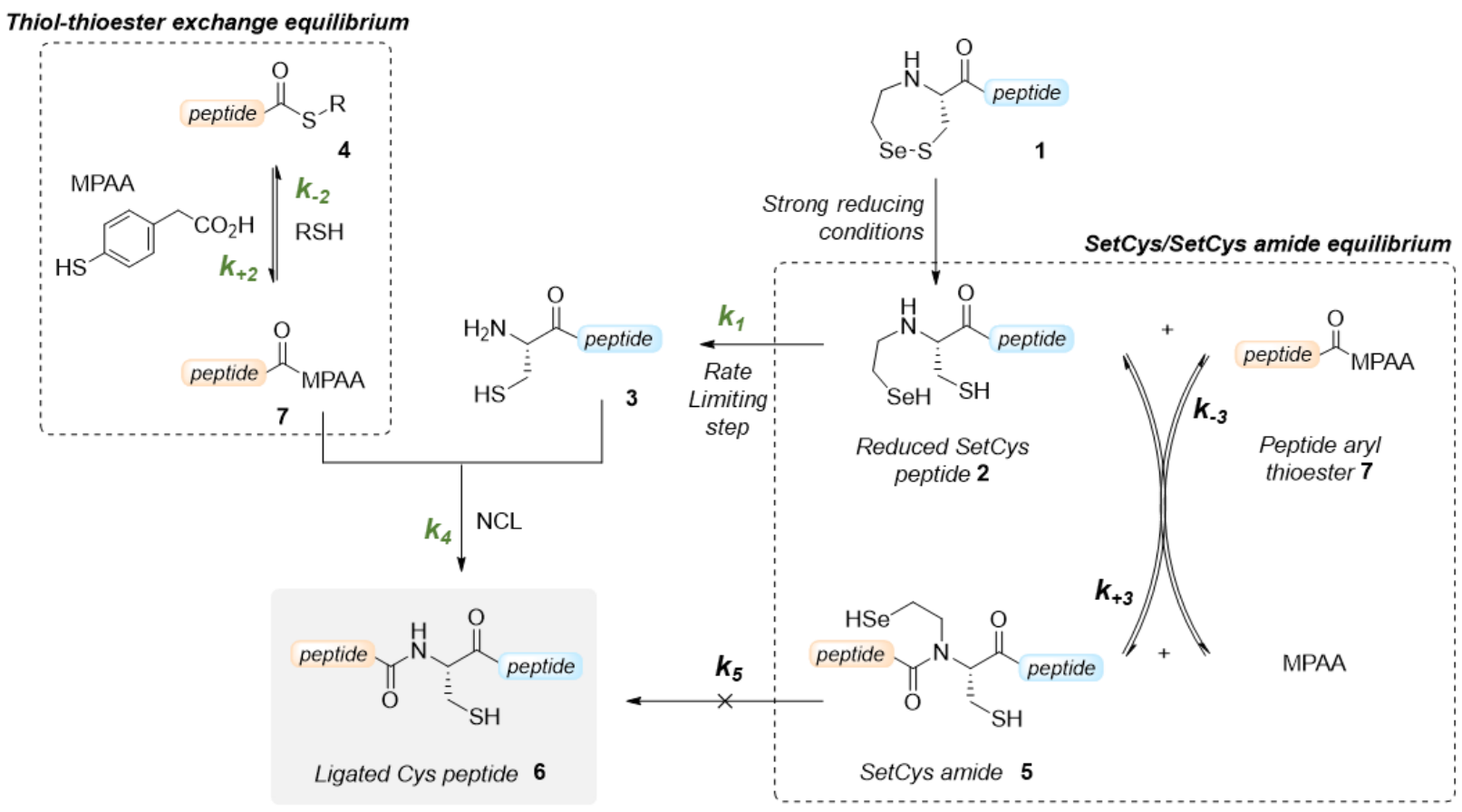

Figure S 103. Mechanistic model for the NCL of SetCys peptides

The purpose of this model is to clarify the following points:

- Determination of rate constants and identification of the rate-limiting step.

- Are the kinetic parameters extracted from the model in accord with experimental data?

- Based on the fact that SetCys peptide $\mathbf{1}$ evolves into Cys peptide 3, is such a reaction also possible from intermediate $\mathbf{5}$ to generate ligated products at Cys of type $\mathbf{6}$ ?

\section{Methodological approach}

In order to determine the rate constants involved in the different steps of this complex process, the system was decomposed into simpler reactions, wherefrom kinetic parameters were extracted using KinteK Explorer Software ${ }^{T M}$ (Version 7.2.180216, Kintek Corporation https://kintekcorp.com/software/).

\subsection{Rate constants for a classical NCL $\left(k_{+2}, k_{-2}\right.$ and $k 4$, see Figure $\left.4 c\right)$}

The kinetic profile of an NCL reaction was first studied in order to determine the rate constants of the thiol-thioester exchange step $\left(\mathrm{k}_{+2}\right.$ and $\left.\mathrm{k}_{-2}\right)$ and of the capture of the transient arylthioester by a cysteinyl peptide $\left(\mathrm{k}_{4}\right)$ (Figure $\left.\mathrm{S} 104\right)$. Importantly, a model peptidyl thioester possessing a C-terminal alanyl residue was used in this study. 


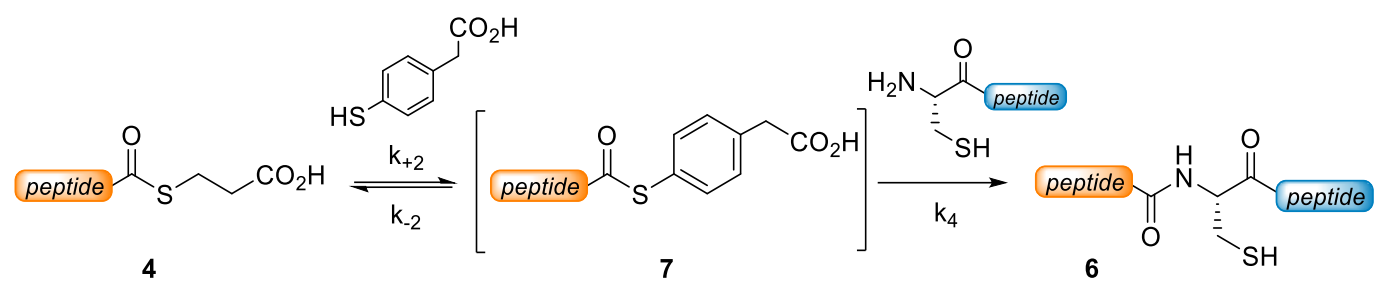

Figure S 104. Kinetic study of the NCL reaction at submillimolar peptide concentration

At the peptide millimolar concentration range, NCL reactions are under the kinetic control of thiol-thioester exchanges. Such conditions do not allow to observe the accumulation of the transient aryl thioester 7 and make the determination of $\mathrm{k}_{4}$ rate constant impossible.

In order to extract second-order rate constants, the thioester and the Cys peptide were reacted at a respective concentration of $0.1 \mathrm{mM}$ and $0.15 \mathrm{mM}$ (in phosphate buffer $0.1 \mathrm{M}, 6 \mathrm{M} \mathrm{Gn} . \mathrm{HCl}$, $200 \mathrm{mM}$ MPAA, $100 \mathrm{mM}$ TCEP, $\mathrm{pH} 7.2,37^{\circ} \mathrm{C}$ ). $50 \mu \mathrm{L}$ of the reaction mixture were collected every $\sim 20$ minutes and added to $50 \mu \mathrm{L}$ of a $50 \%$ acetic acid aqueous solution. The sample was extracted three times by $\mathrm{Et}_{2} \mathrm{O}$ and the aqueous layer was analyzed by RP-HPLC (eluent A $0.1 \%$ TFA in water, eluent B $0.1 \%$ TFA in $\mathrm{CH}_{3} \mathrm{CN} /$ water: $4 / 1$ by vol. $\mathrm{C} 18$ Xbridge $\mathrm{BEH} 300 \AA 5 \mu \mathrm{m}$ $(4.6 \times 250 \mathrm{~mm})$ column, gradient $0-50 \% \mathrm{~B}$ in $15 \mathrm{~min}, 1 \mathrm{~mL} / \mathrm{min}, 30^{\circ} \mathrm{C}$, detection at $\left.215 \mathrm{~nm}\right)$

The chromatograms were processed on the basis of the absorbance signal at $215 \mathrm{~nm}$. All datasets were fitted with an exponential analytical function to obtain an estimate of the standard deviation. A subsequent global fit allowed determining rate constants $\mathrm{k}_{+2}, \mathrm{k}_{-2}, \mathrm{k}_{4}$ (Table S 4 and Figure S 105).

Table S 4. Second-order rate constants for the model NCL reaction

\begin{tabular}{ccc} 
& value $\left(\mathbf{M}^{\mathbf{- 1}} \cdot \mathbf{m i n}^{-\mathbf{1}}\right)$ & Std error \\
\hline $\mathbf{k}+\mathbf{2}$ & 0.257 & 0.006 \\
$\mathbf{k}-\mathbf{2}$ & 129 & 17 \\
$\mathbf{k} 4$ & 243 & 3 \\
\hline
\end{tabular}




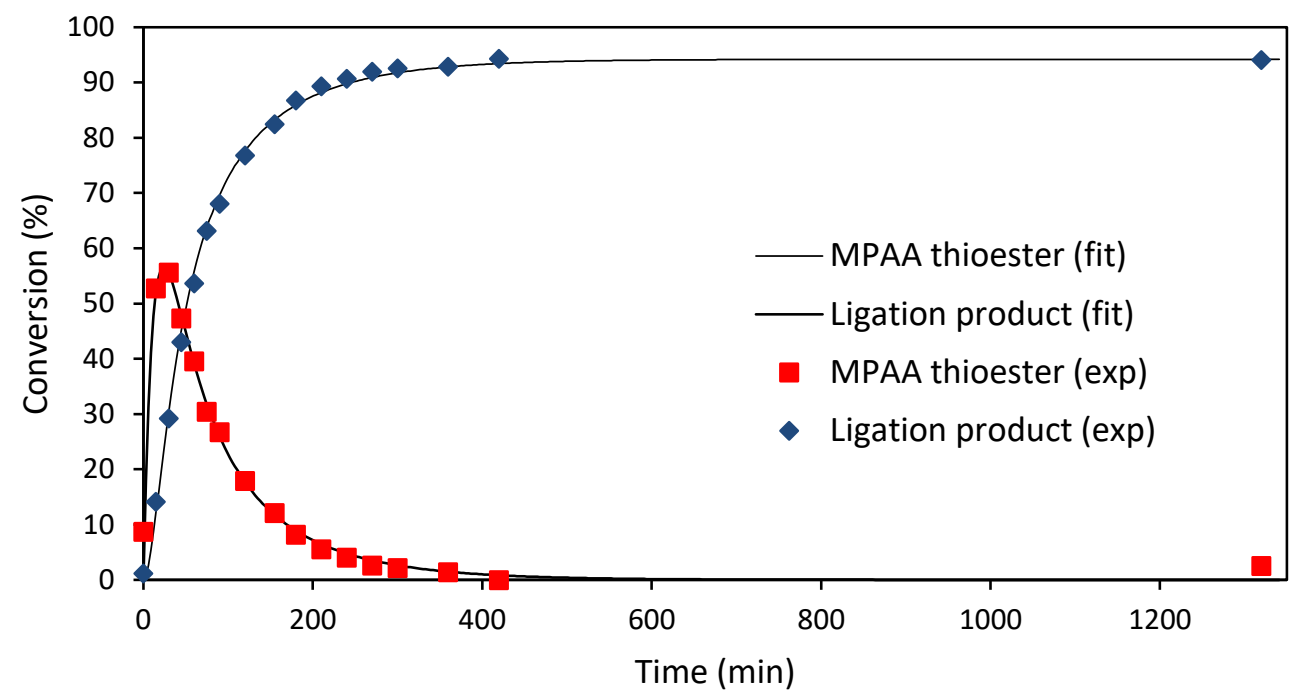

Figure S 105. Fitting of the NCL reaction at submillimolar concentration of peptides using KinteK Explorer Software ${ }^{T M}$ (Version 7.2.180216., Kintek Corporation https://kintekcorp.com/software/). Red squares / Blue diamonds correspond to experimental values.

\subsection{Rate constant for the loss of the $N$-selenoethyl appendage $\left(k_{1}\right.$, Figure $\left.4 c\right)$}

The experimental approach for this reaction is documented in full in section 4.1 of the SI. Note that the reduction of SetCys peptide 1 by TCEP was neglected since this reaction proceeds almost instantly at $\mathrm{pH} 7.2$.

As previously determined, $\mathbf{k}_{1}=\mathbf{0 . 0 0 1 1 9} \pm \mathbf{0 . 0 0 0 8 0} \mathrm{min}^{-1}$ at $\mathbf{p H ~ 7 . 2 , 3 7}{ }^{\circ} \mathrm{C}$.

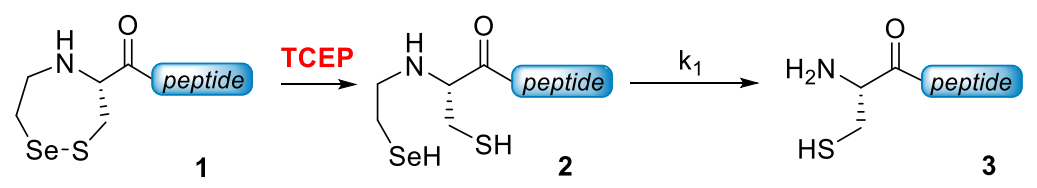

Figure S 106. Mechanistic model for the loss of the $N$-selenoethyl appendage on SetCys peptides.

\subsection{Kinetic model for SetCys-mediated NCL (Figure 4b,c)}

The rate constants determined in the model NCL and arm loss reactions (i.e. $\mathrm{k}_{+2}, \mathrm{k}_{-2}, \mathrm{k}_{1}, \mathrm{k}_{4}$ ) were used to fit kinetic data collected for the NCL of SetCys peptides (Figure S 107) with the model proposed in Figure $\mathrm{S} 103$ and to determine $\mathrm{k}_{+3}$, $\mathrm{k}_{-3}$ and $\mathrm{k}_{5}$ (Table $\mathrm{S} 5$ ). 


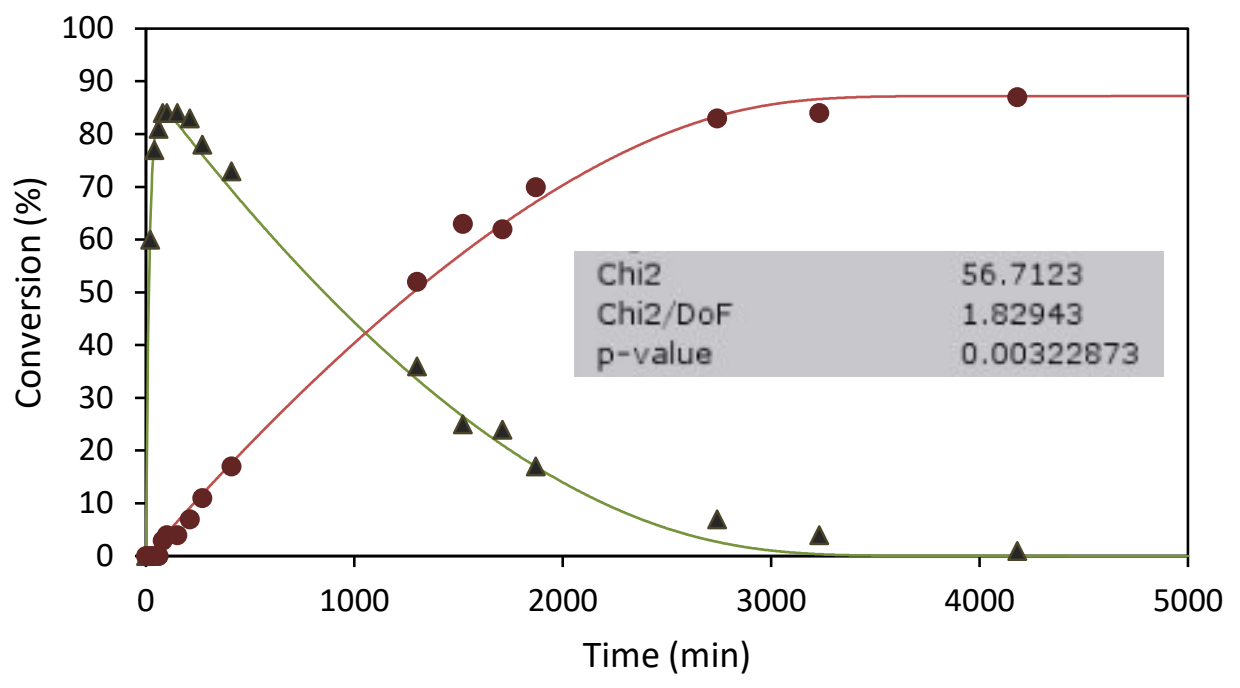

Figure S 107. Ligation of SetCys peptide 1 with MPA-thioester 4 in NCL standard conditions. RP-HPLC monitoring of the formation of peptides 5 (black triangles) and $\mathbf{6}$ (brown circles) throughout the course of the reaction. The continuous lines correspond to the fitting of experimental data using KinteK Explorer Software ${ }^{T M}$ (Version 7.2.180216., Kintek Corporation - https://kintekcorp.com/software/).

Table S 5. Rate constants for the model NCL reaction of SetCys peptides.

\begin{tabular}{ccc} 
& Value & Std err \\
\hline $\mathbf{k} 1$ & $0.0012 \mathrm{~min}^{-1}$ & 0.0008 \\
$\mathbf{k}+\mathbf{2}$ & $0.257 \mathrm{M}^{-1} \cdot \mathrm{min}^{-1}$ & 0.006 \\
$\mathbf{k}-\mathbf{2}$ & $129 \mathrm{M}^{-1} \cdot \mathrm{min}^{-1}$ & 17 \\
$\mathbf{k}+\mathbf{3}$ & $182 \mathrm{M}^{-1} \cdot \mathrm{min}^{-1}$ & 49 \\
$\mathbf{k} \mathbf{3}$ & $0.0632 \mathrm{M}^{-1} \cdot \mathrm{min}^{-1}$ & 0.0314 \\
$\mathbf{k} 4$ & $243 \mathrm{M}^{-1} \cdot \mathrm{min}^{-1}$ & 3 \\
$\mathbf{k} 5$ & $0 \mathrm{~min}^{-1}$ & $\mathrm{nd}$
\end{tabular}

Note that according to the proposed model, best fit is obtained for a value of $\mathrm{k}_{5}$ being null which excludes the loss of the $\mathrm{N}$-selenoethyl appendage from the ligated product. 


\section{Synthesis of cyclic peptides (Figure 5a,b)}
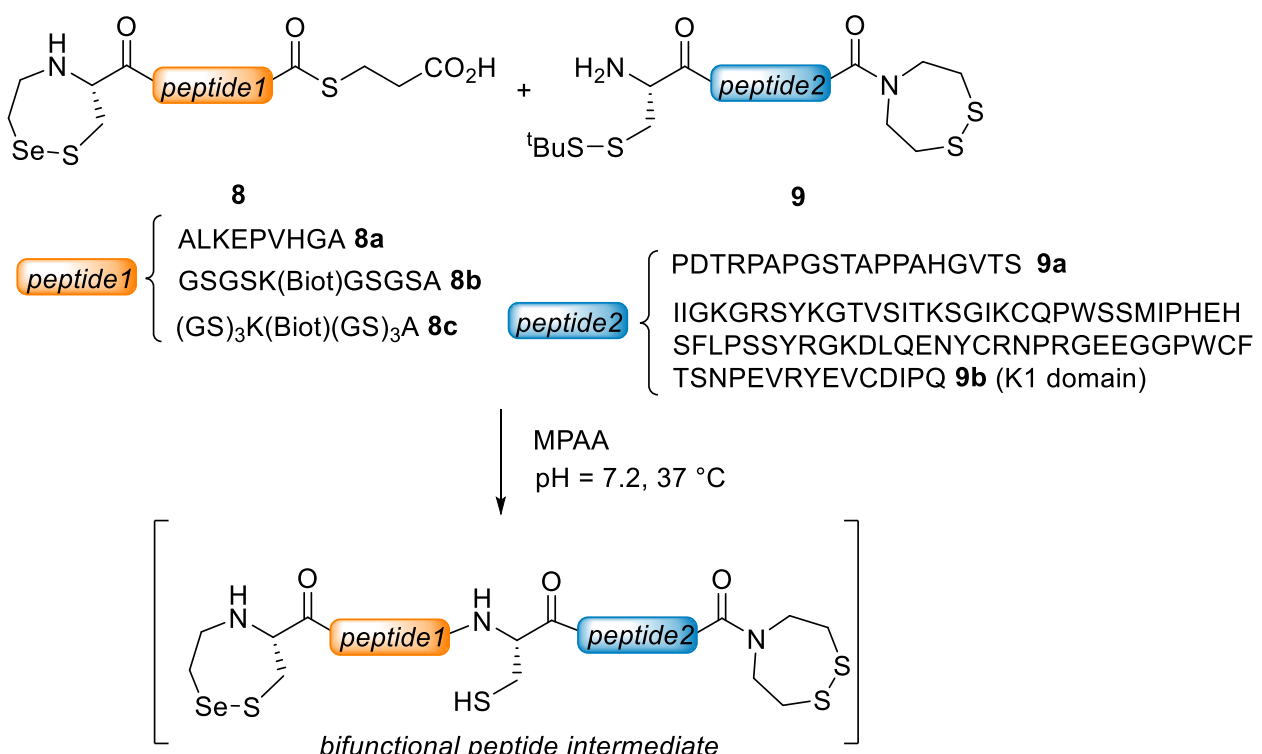

bifunctional peptide intermediate

MPAA, TCEP

ascorbate

$\mathrm{pH}=5.5,37^{\circ} \mathrm{C}$

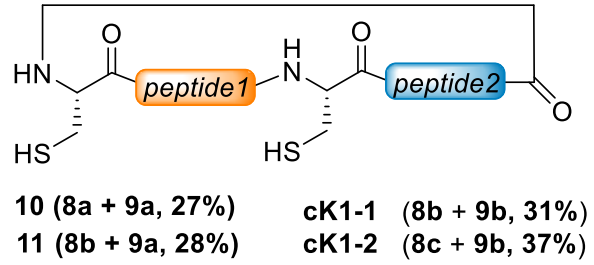

Figure S 108. One-pot synthesis of cyclic peptides 10, 11, cK1-1 and cK1-2.

\subsection{Synthesis of cyclic peptide 10}

To a solution of $\mathrm{Gn} \cdot \mathrm{HCl}(287 \mathrm{mg})$ in $0.1 \mathrm{M}$, pH 7.4 phosphate buffer $(300 \mu \mathrm{L})$ was added MPAA $(16.8 \mathrm{mg})$ and the $\mathrm{pH}$ of the mixture was adjusted to 7.20 by addition of $6 \mathrm{M} \mathrm{NaOH}$. Peptide 9a (4.50 mg, $1.82 \mu \mathrm{mol}, 1 \mathrm{eq})$ and peptide 8a (2.84 mg, $1.82 \mu \mathrm{mol}, 1 \mathrm{eq})$ were then successively dissolved in the MPAA solution $(228 \mu \mathrm{L})$ and the mixture was stirred at $37{ }^{\circ} \mathrm{C}$. The progress of the reaction leading to the bifunctional peptide intermediate was followed by HPLC (Figure S 109).

After completion of the NCL ligation $(3 \mathrm{~h})$, the second step of the process leading to the cyclisation of the peptide was induced by addition of TCEP. To a solution of $\mathrm{Gn} \cdot \mathrm{HCl}(1.32 \mathrm{~g})$ in $0.1 \mathrm{M}, \mathrm{pH} 7.4$ phosphate buffer $(1.38 \mathrm{~mL})$ were added TCEP. $\mathrm{HCl}(87.7 \mathrm{mg})$, sodium ascorbate $(60.6 \mathrm{mg})$ and MPAA $(77.4 \mathrm{mg})$ and the $\mathrm{pH}$ of the mixture was adjusted to 5.51 by addition of $6 \mathrm{M} \mathrm{NaOH}$. The previous ligation mixture containing the bifunctional peptide 
intermediate was then diluted with the solution of TCEP $(2.05 \mathrm{~mL})$ and the mixture was stirred at $37{ }^{\circ} \mathrm{C}$. After completion of the reaction $(40 \mathrm{~h})$, the mixture was acidified with $10 \% \mathrm{AcOH}$ in water $(5 \mathrm{~mL})$ and diluted with water $(2.5 \mathrm{~mL})$. The mixture was then extracted with $\mathrm{Et}_{2} \mathrm{O}(5$ $x$ ) to remove the MPAA and purified by HPLC (eluent $\mathrm{A}=$ water containing $0.1 \%$ TFA, eluent $\mathrm{B}=$ acetonitrile in water $4 / 1$ containing $0.1 \% \mathrm{TFA}, 50^{\circ} \mathrm{C}$, detection at $215 \mathrm{~nm}, 6 \mathrm{~mL} / \mathrm{min}, 0-10 \%$ eluent $\mathrm{B}$ in $5 \mathrm{~min}$, then 10-35\% eluent $\mathrm{B}$ in $30 \mathrm{~min}, \mathrm{C} 18 \mathrm{XBridge}$ column) to give the cyclic peptide $\mathbf{1 0}$ as a with solid after lyophilization (1.65 mg, 27\%).
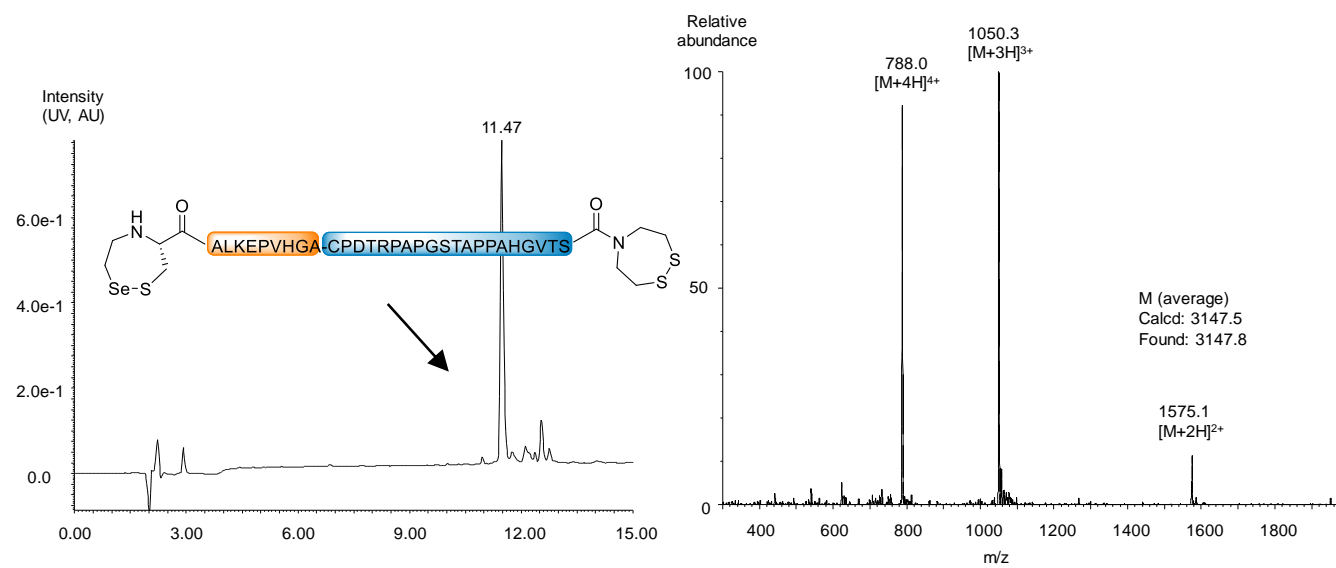

b)
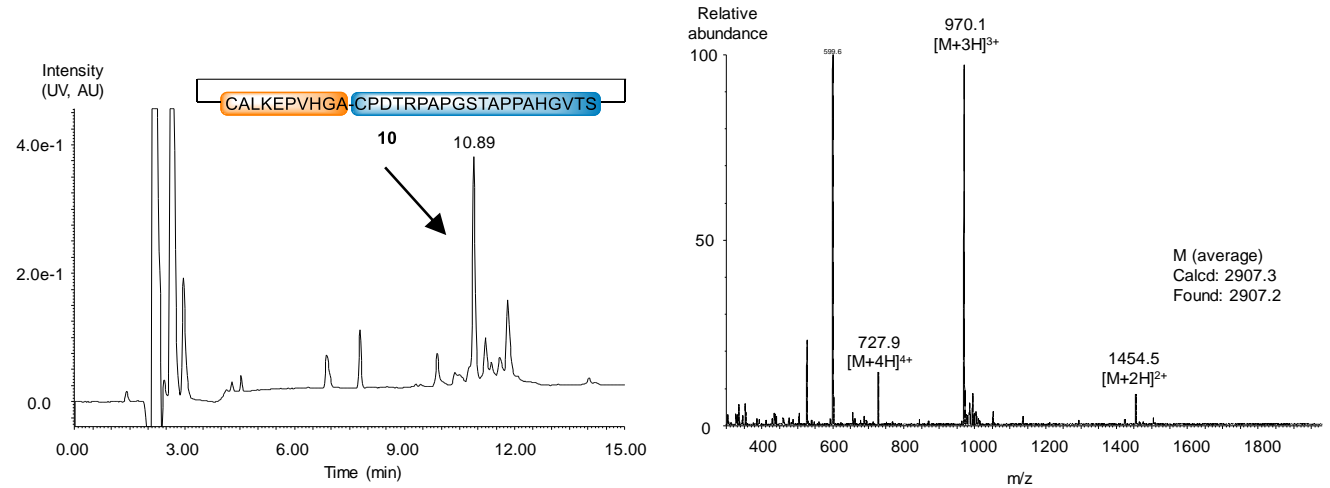

c)

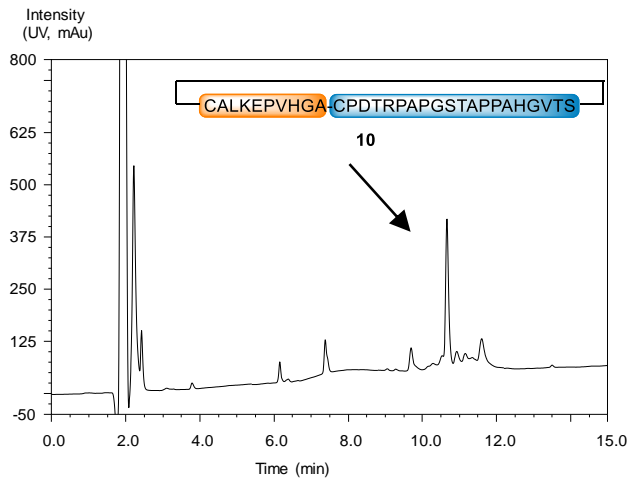

Figure S 109. Assembly of the cyclic peptide 10 monitored by LC-MS (a and b) and HPLC (c). a) Analysis of the reaction mixture 190 min after mixing the two partners of the ligation reaction. b) Analysis of the reaction mixture $16 \mathrm{~h}$ after the addition of TCEP. c) Analysis of the reaction mixture $22 \mathrm{~h}$ after the addition of TCEP. LC trace: eluent A $0.10 \%$ TFA in water, eluent $\mathrm{B} 0.10 \%$ TFA in $\mathrm{CH}_{3} \mathrm{CN} /$ water: $4 / 1$ by vol., $\mathrm{C} 18$ column, gradient $0-50 \%$ B in 15 min, $50{ }^{\circ} \mathrm{C}$ (for a and b) or $30{ }^{\circ} \mathrm{C}$ (for c), $1 \mathrm{~mL} / \mathrm{min}$, UV detection at $215 \mathrm{~nm}$. 


\section{CALKEPVHGA-CPDTRPAPGSTAPPAHGVTS}

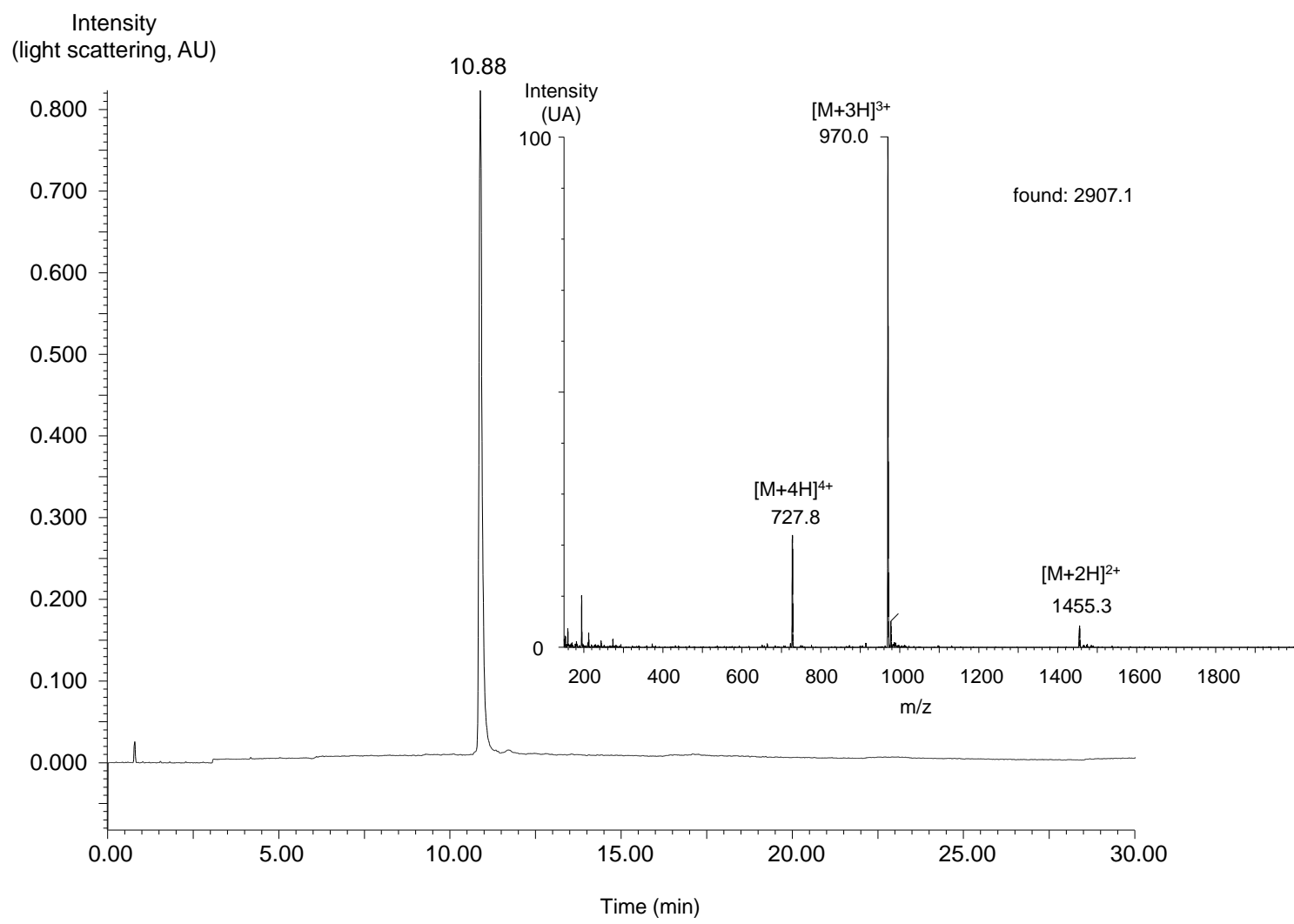

Figure S 110. LC-MS analysis of purified cyclic peptide 10. LC trace: eluent A $0.10 \%$ TFA in water, eluent $\mathrm{B} 0.10 \%$ TFA in $\mathrm{CH}_{3} \mathrm{CN} /$ water: $4 / 1$ by vol. $\mathrm{C} 18$ column, gradient $0-100 \% \mathrm{~B}$ in $30 \mathrm{~min}, 50{ }^{\circ} \mathrm{C}, 1 \mathrm{~mL} / \mathrm{min}$, light scattering detection. MS trace: $\mathrm{m} / \mathrm{z} 1455.3\left([\mathrm{M}+2 \mathrm{H}]^{2+}\right), 970.0$ $\left([\mathrm{M}+3 \mathrm{H}]^{3+}\right), 727.8\left([\mathrm{M}+4 \mathrm{H}]^{+}\right)$; calcd. for M (average): 2907.3, found: 2907.1. 


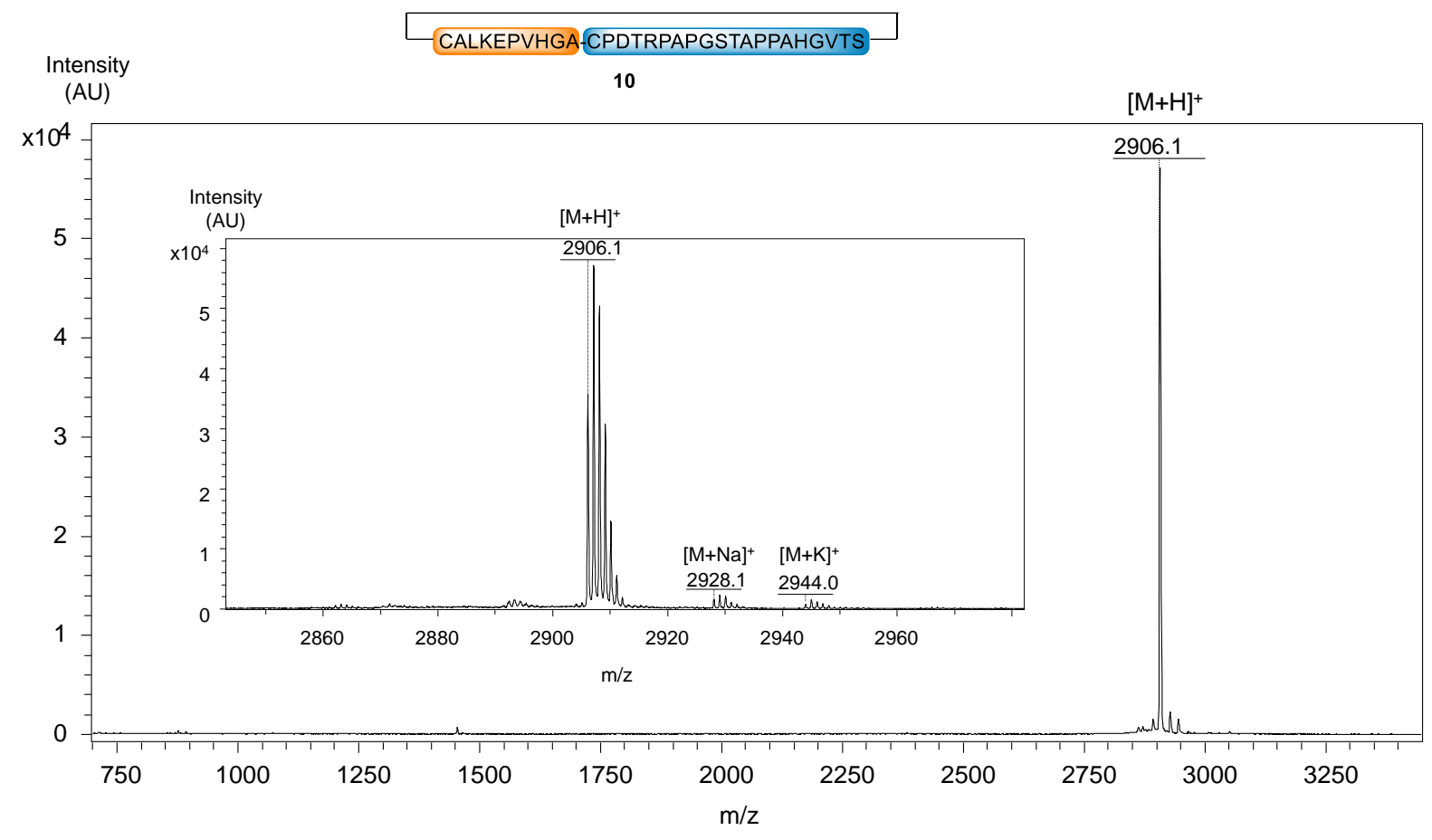

Figure S 111. MALDI-TOF analysis of cyclic peptide 10. Matrix $\alpha$-cyano-4-hydroxycinnamic acid, positive detection mode, calcd. for $[\mathrm{M}+\mathrm{H}]^{+}$(monoisotopic): 2906.40, found: 2906.1 .

\subsection{Synthesis of cyclic peptide 11}

To a solution of $\mathrm{Gn} \cdot \mathrm{HCl}(287 \mathrm{mg})$ in $0.1 \mathrm{M}, \mathrm{pH} 7.4$ phosphate buffer $(300 \mu \mathrm{L})$ was added MPAA $(16.8 \mathrm{mg})$ and the $\mathrm{pH}$ of the mixture was adjusted to 7.20 by addition of $6 \mathrm{M} \mathrm{NaOH}$. peptide 9a (4.50 mg, $1.82 \mu \mathrm{mol}, 1 \mathrm{eq})$ and peptide $\mathbf{8 b}(2.61 \mathrm{mg}, 1.82 \mu \mathrm{mol}, 1 \mathrm{eq})$ were then successively dissolved in the MPAA solution $(228 \mu \mathrm{L})$ and the mixture was stirred at $37{ }^{\circ} \mathrm{C}$. The reaction yielding the bifunctional peptide intermediate was monitored by HPLC.

After completion of the NCL ligation $(3.5 \mathrm{~h})$, the second step of the process leading to the cyclisation of the peptide was induced by addition of TCEP. To a solution of $\mathrm{Gn} \cdot \mathrm{HCl}(1.32 \mathrm{~g})$ in $0.1 \mathrm{M}$, pH 7.4 phosphate buffer $(1.38 \mathrm{~mL})$ were added TCEP·HCl $(87.7 \mathrm{mg})$, sodium ascorbate $(60.6 \mathrm{mg})$ and MPAA $(77.4 \mathrm{mg})$ and the $\mathrm{pH}$ of the mixture was adjusted to 5.50 by addition of $6 \mathrm{M} \mathrm{NaOH}$. The previous ligation mixture containing the bifunctional peptide intermediate was then diluted with the solution of TCEP $(2.05 \mathrm{~mL})$ and the mixture was stirred at $37{ }^{\circ} \mathrm{C}$. After completion of the reaction (4 days), the mixture was acidified with $10 \% \mathrm{AcOH}$ in water $(7.5 \mathrm{~mL})$ and diluted with water $(10 \mathrm{~mL})$. The crude was then extracted with $\mathrm{Et}_{2} \mathrm{O}(5$ $x$ ) to remove the MPAA and purified by HPLC (eluent $\mathrm{A}=$ water containing $0.1 \%$ TFA, eluent $\mathrm{B}=$ acetonitrile in water $4 / 1$ containing $0.1 \% \mathrm{TFA}, 50^{\circ} \mathrm{C}$, detection at $215 \mathrm{~nm}, 6 \mathrm{~mL} / \mathrm{min}, 0-5 \%$ eluent $\mathrm{B}$ in $3 \mathrm{~min}$, then $5-35 \%$ eluent $\mathrm{B}$ in $32 \mathrm{~min}, \mathrm{C} 18 \mathrm{XBridge}$ column) to give the cyclic peptide 11 as a with solid after lyophilization $(1.65 \mathrm{mg}, 28 \%)$. 

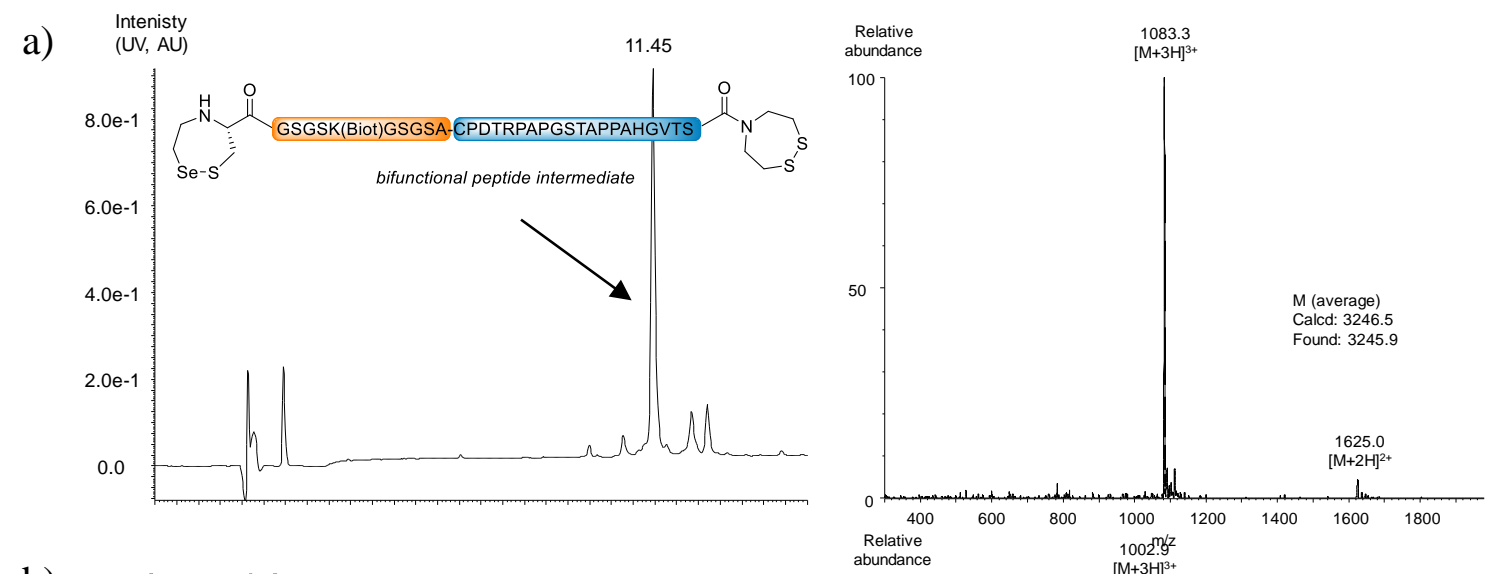

b)
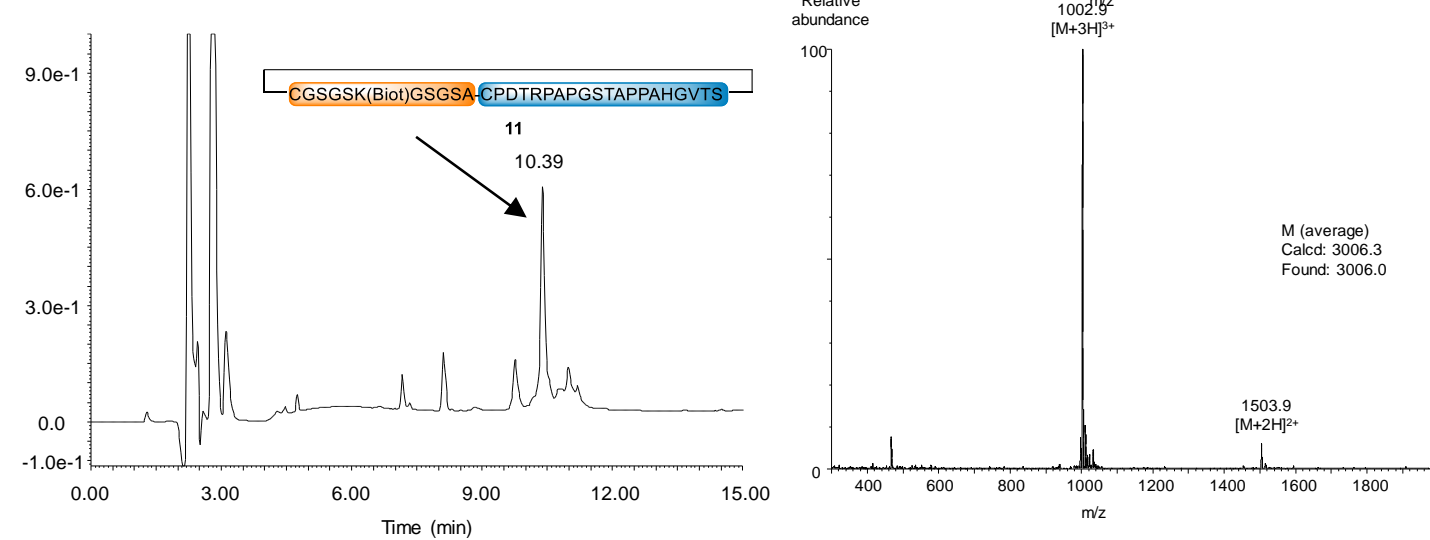

Figure S 112. Assembly of the cyclic peptide 11 monitored by LC-MS. a) Analysis of the reaction mixture $190 \mathrm{~min}$ after mixing the two partners of the ligation reaction. b) Analysis of the reaction mixture $4 \mathrm{~d}$ after the dilution with TCEP solution. LC trace: eluent A $0.10 \%$ TFA in water, eluent $\mathrm{B} 0.10 \%$ TFA in $\mathrm{CH}_{3} \mathrm{CN} /$ water: $4 / 1$ by vol. $\mathrm{C} 18$ column, gradient $0-50 \% \mathrm{~B}$ in $15 \mathrm{~min}, 50{ }^{\circ} \mathrm{C}, 1 \mathrm{~mL} / \mathrm{min}$, UV detection at $215 \mathrm{~nm}$. 


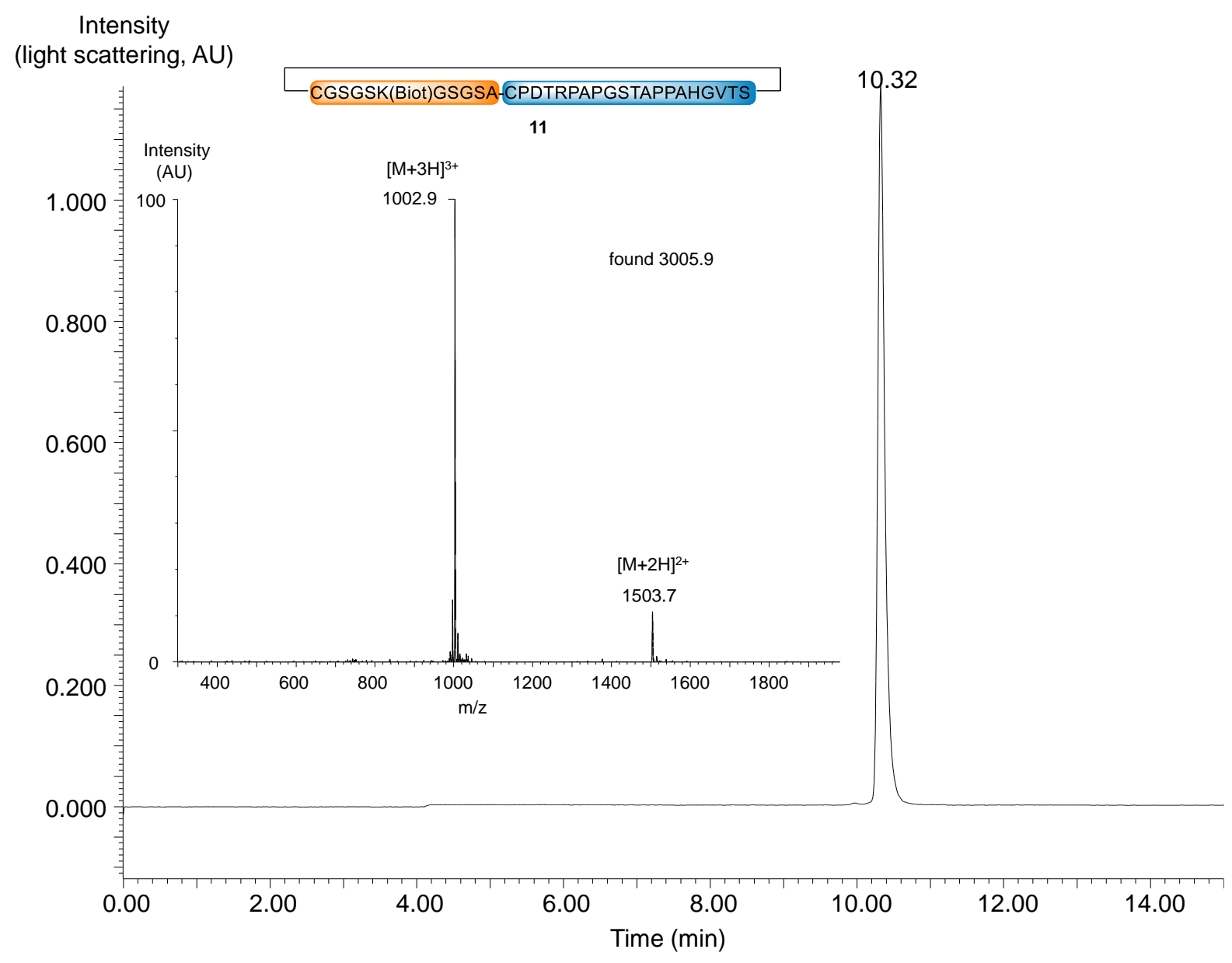

Figure S 113. LC-MS analysis of purified cyclic peptide 11. LC trace: eluent A $0.10 \%$ TFA in water, eluent $\mathrm{B} 0.10 \%$ TFA in $\mathrm{CH}_{3} \mathrm{CN} /$ water: $4 / 1$ by vol. $\mathrm{C} 18$ Xbridge $\mathrm{BEH} 300 \AA 5 \mu \mathrm{m}(4.6$ $\times 250 \mathrm{~mm}$ ) column, $30{ }^{\circ} \mathrm{C}$, gradient $0-50 \% \mathrm{~B}$ in $15 \mathrm{~min}, 1 \mathrm{~mL} / \mathrm{min}$, light scattering detection. MS trace: $\mathrm{m} / \mathrm{z}=1503.7\left([\mathrm{M}+2 \mathrm{H}]^{2+}\right), 1002.9\left([\mathrm{M}+3 \mathrm{H}]^{3+}\right)$; calcd for $\mathrm{M}: 3006.3$, found: 3005.9 . 


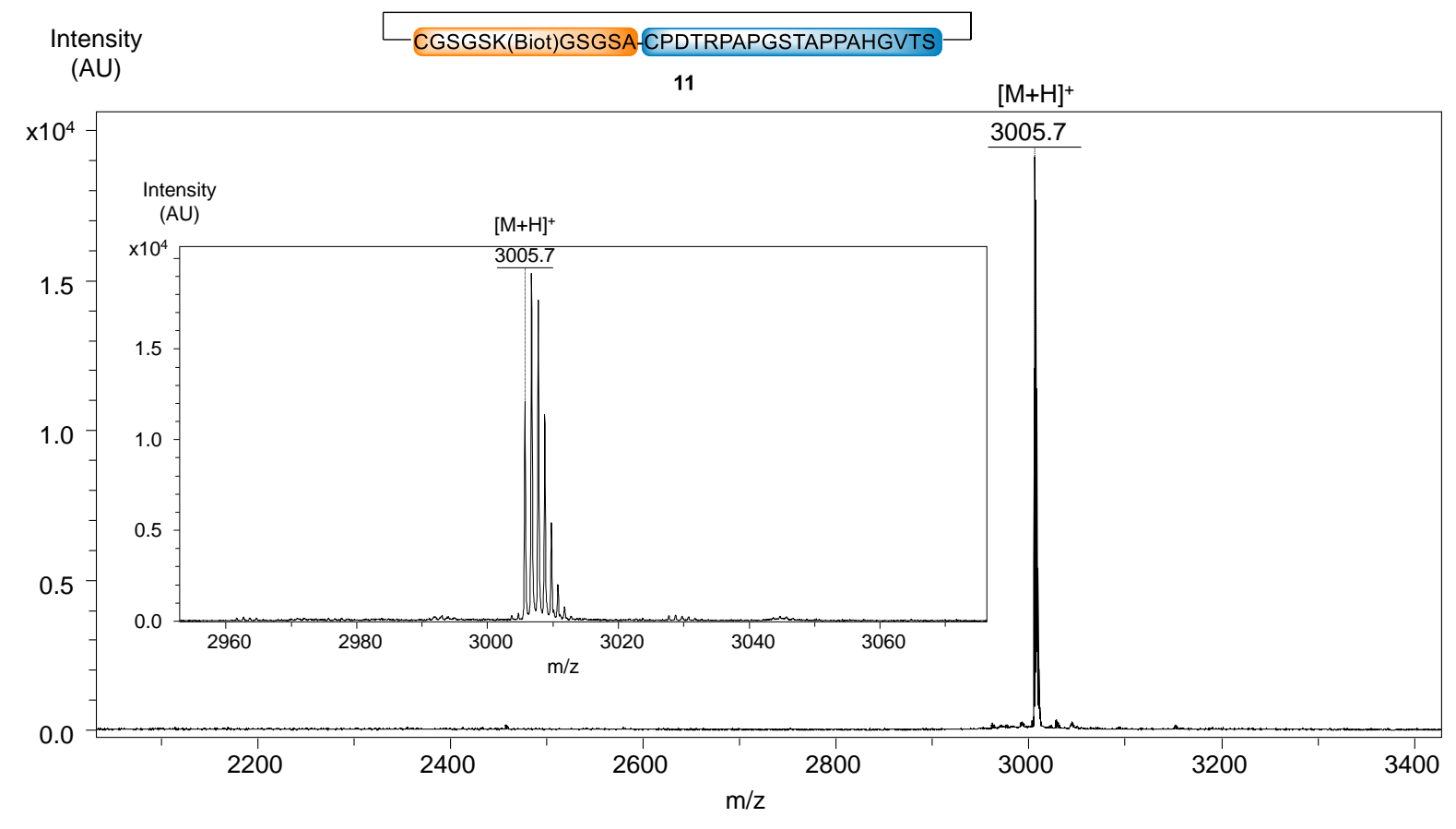

Figure S 114. MALDI-TOF analysis of cyclic peptide 11. Matrix $\alpha$-cyano-4-hydroxycinnamic acid, positive detection mode, calcd. for $[\mathrm{M}+\mathrm{H}]^{+}$(monoisotopic): 3005.32, found: 3005.7.

\subsection{Synthesis of cK1-1}

To a solution of $\mathrm{Gn} \cdot \mathrm{HCl}(287 \mathrm{mg})$ in $0.1 \mathrm{M}, \mathrm{pH} 7.4$ phosphate buffer $(300 \mu \mathrm{L})$ was added MPAA $(16.8 \mathrm{mg})$ and the $\mathrm{pH}$ of the mixture was adjusted to 7.25 by addition of $6 \mathrm{M} \mathrm{NaOH}$. Peptide 9b (5.03 mg, $0.481 \mu \mathrm{mol}, 1$ eq) and peptide $8 \mathbf{b}(0.688 \mathrm{mg}, 0.481 \mu \mathrm{mol}, 1 \mathrm{eq})$ were successively dissolved in the MPAA solution $(120 \mu \mathrm{L})$ and the mixture was stirred at $37{ }^{\circ} \mathrm{C}$. The progress of the ligation leading to the bifunctional peptide intermediate was followed by HPLC (Figure S 115).

After completion of the NCL ligation $(3.5 \mathrm{~h})$, the second step of the process leading to the cyclisation of the peptide was induced by addition of TCEP. To a solution of $\mathrm{Gn} \cdot \mathrm{HCl}(1.72 \mathrm{~g})$ in $0.1 \mathrm{M}, \mathrm{pH} 7.4$ phosphate buffer $(1.80 \mathrm{~mL})$ were added TCEP·HCl $(90.2 \mathrm{mg})$, sodium ascorbate $(63 \mathrm{mg})$ and MPAA $(100.3 \mathrm{mg}$ ) and the $\mathrm{pH}$ of the mixture was adjusted to 5.51 by addition of $6 \mathrm{M} \mathrm{NaOH}$. The previous ligation mixture containing the bifunctional peptide intermediate was then diluted with the solution of TCEP $(2.28 \mathrm{~mL})$ and the mixture was stirred at $37^{\circ} \mathrm{C}$ for $40 \mathrm{~h}$. After completion of the reaction, the mixture was acidified with $\mathrm{AcOH}(0.30$ $\mathrm{mL})$ and extracted with $\mathrm{Et}_{2} \mathrm{O}(5 \times)$ to remove the MPAA. The crude was further diluted with water $(9 \mathrm{~mL}$ ) and purified by HPLC (eluent $\mathrm{A}=$ water containing $0.1 \% \mathrm{TFA}$, eluent $\mathrm{B}=$ acetonitrile in water $4 / 1$ containing $0.1 \% \mathrm{TFA}, 50^{\circ} \mathrm{C}$, detection at $215 \mathrm{~nm}, 6 \mathrm{~mL} / \mathrm{min}, 0-20 \%$ eluent B in $5 \mathrm{~min}$, then $20-45 \%$ eluent $\mathrm{B}$ in $60 \mathrm{~min}, \mathrm{C} 18 \mathrm{XBridge}$ column) to give the cyclic peptide cK1-1 as a white solid after lyophilization (1.71 $\mathrm{mg}, 31 \%)$. 
a)
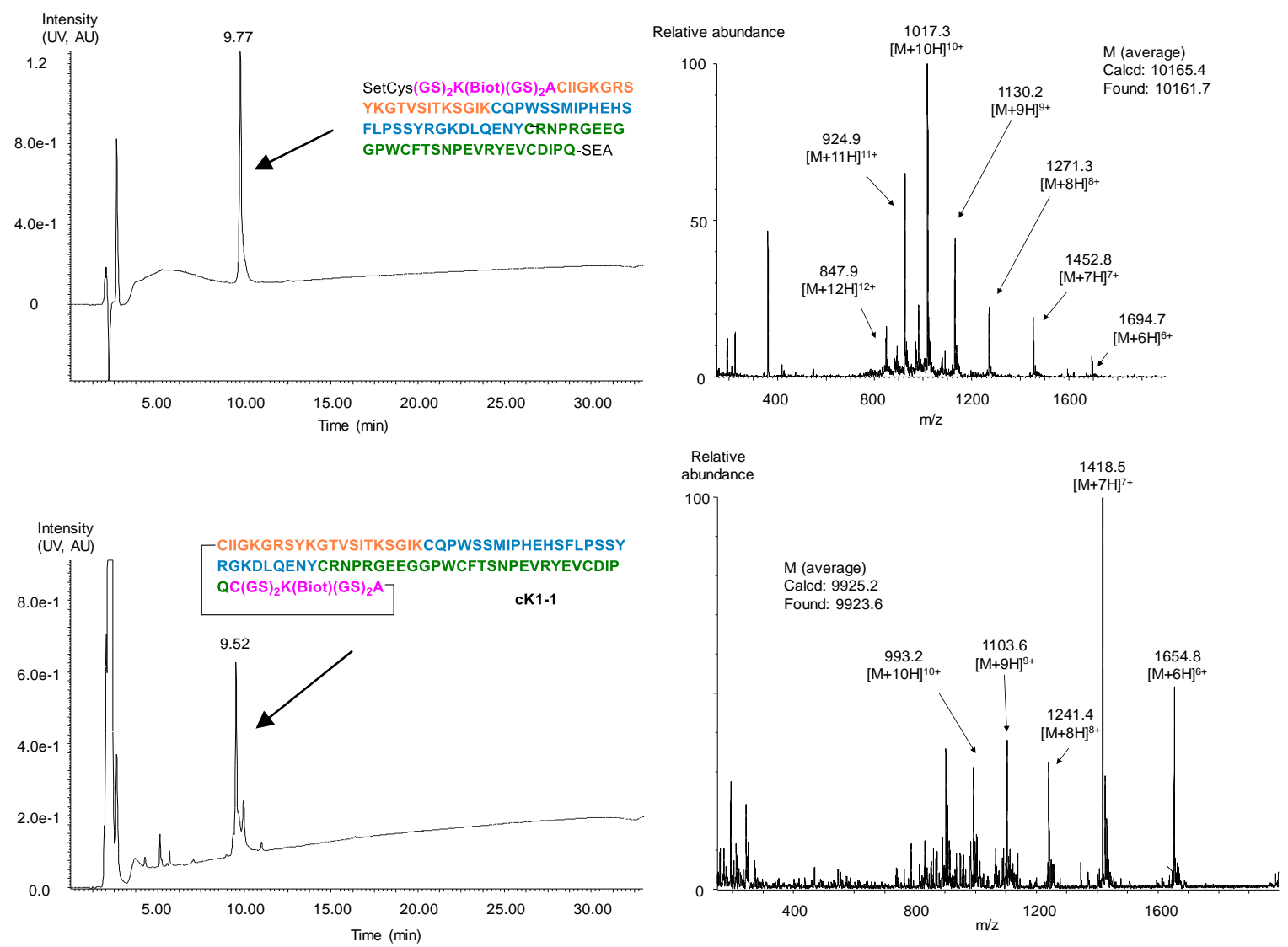

Figure S 115. Assembly of cK1-1 monitored by LC-MS. a) Analysis of the reaction mixture 120 min after mixing the two partners of the ligation reaction. b) Analysis of the reaction mixture $40 \mathrm{~h}$ after the addition of TCEP. LC trace: eluent A $0.10 \%$ TFA in water, eluent B $0.10 \%$ TFA in $\mathrm{CH}_{3} \mathrm{CN} /$ water: $4 / 1$ by vol., $\mathrm{C} 18$ column, gradient $0-100 \% \mathrm{~B}$ in $30 \mathrm{~min}, 50{ }^{\circ} \mathrm{C}, 1$ $\mathrm{mL} / \mathrm{min}$, UV detection at $215 \mathrm{~nm}$. 
a)

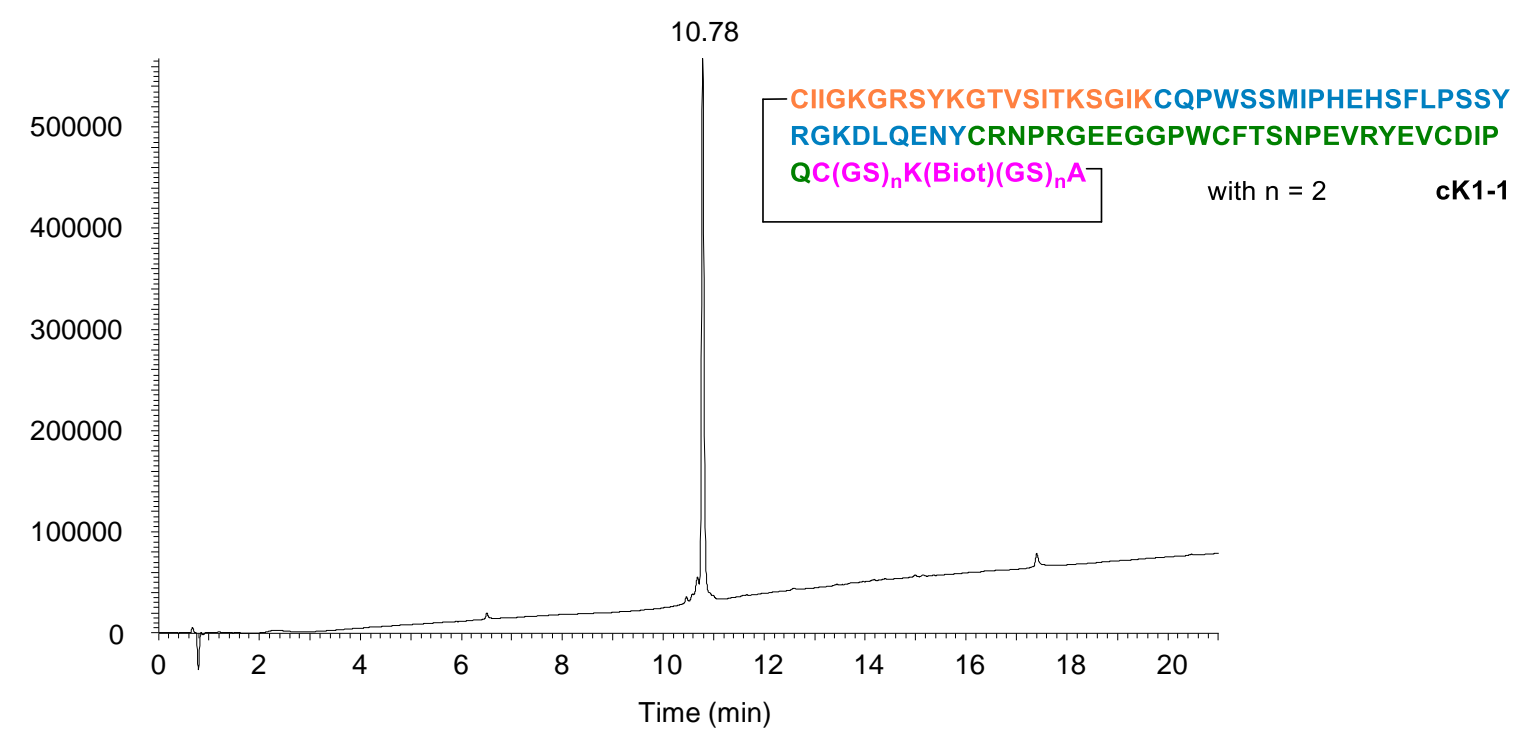

b)

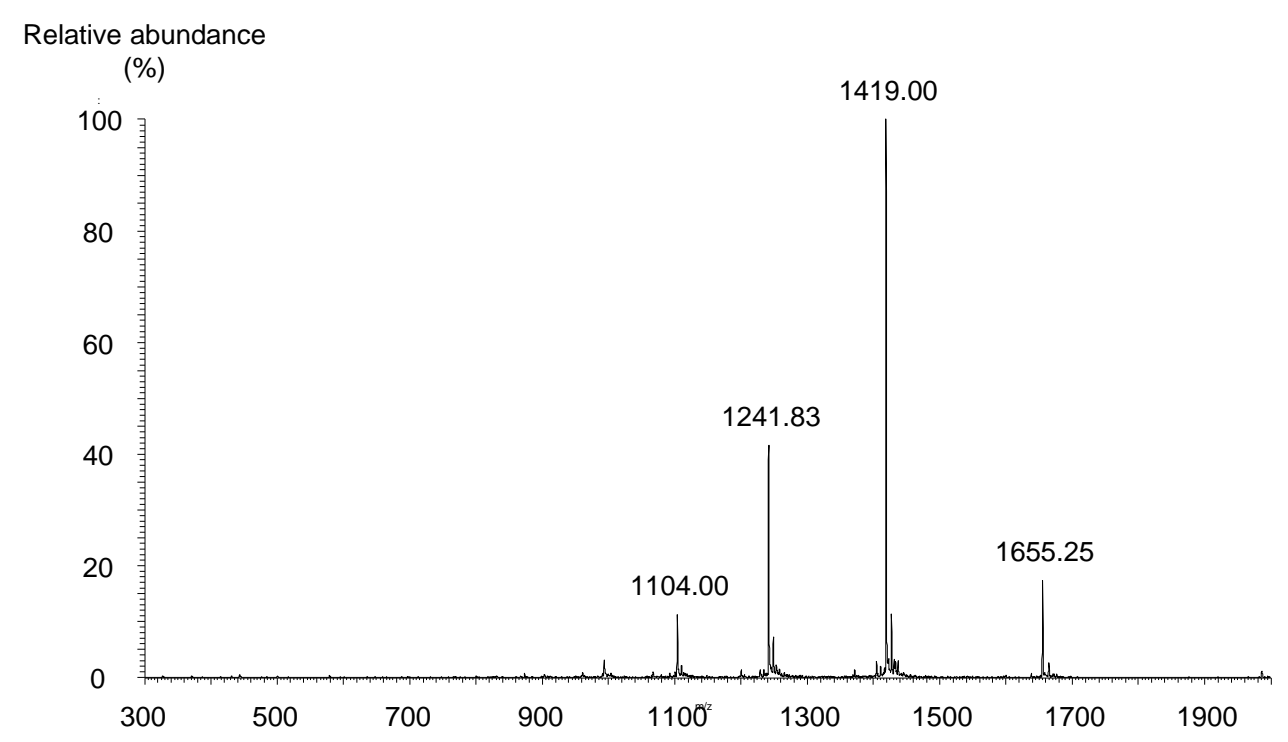

Figure S 116. LC-MS analysis of cK1-1. a) LC trace. Eluent A $0.10 \%$ TFA in water, eluent B $0.10 \%$ TFA in $\mathrm{CH}_{3} \mathrm{CN}$, ACQUITY UPLC Peptide BEH $300 \AA 1.7 \mu \mathrm{m}(2.1 \times 150 \mathrm{~mm})$ column, $50{ }^{\circ} \mathrm{C}$, gradient $0-70 \% \mathrm{~B}$ in $20 \mathrm{~min}, 0.4 \mathrm{~mL} / \mathrm{min}$, UV detection. b) MS trace: $\mathrm{m} / \mathrm{z}=1655.25$ $\left([\mathrm{M}+6 \mathrm{H}]^{6+}\right), 1419.00\left([\mathrm{M}+7 \mathrm{H}]^{7+}\right), 1241.83\left([\mathrm{M}+8 \mathrm{H}]^{8+}\right), 1104.00\left([\mathrm{M}+10 \mathrm{H}]^{10+}\right)$; calcd. for M (average): 9925.19, found: 9926.28.

\subsubsection{Characterization of the cyclic backbone structure of cK1-1}

\section{Reduction-alkylation}

cK1-1 $(50 \mu \mathrm{g})$ was treated with a solution of iodoacetamide in $0.025 \mathrm{M}$ ammonium bicarbonate $(10 \mathrm{mg} / \mathrm{mL}, 50 \mu \mathrm{L})$ for $15 \mathrm{~min}$. The alkylation step was monitored by MALDI-TOF mass spectrometry. 

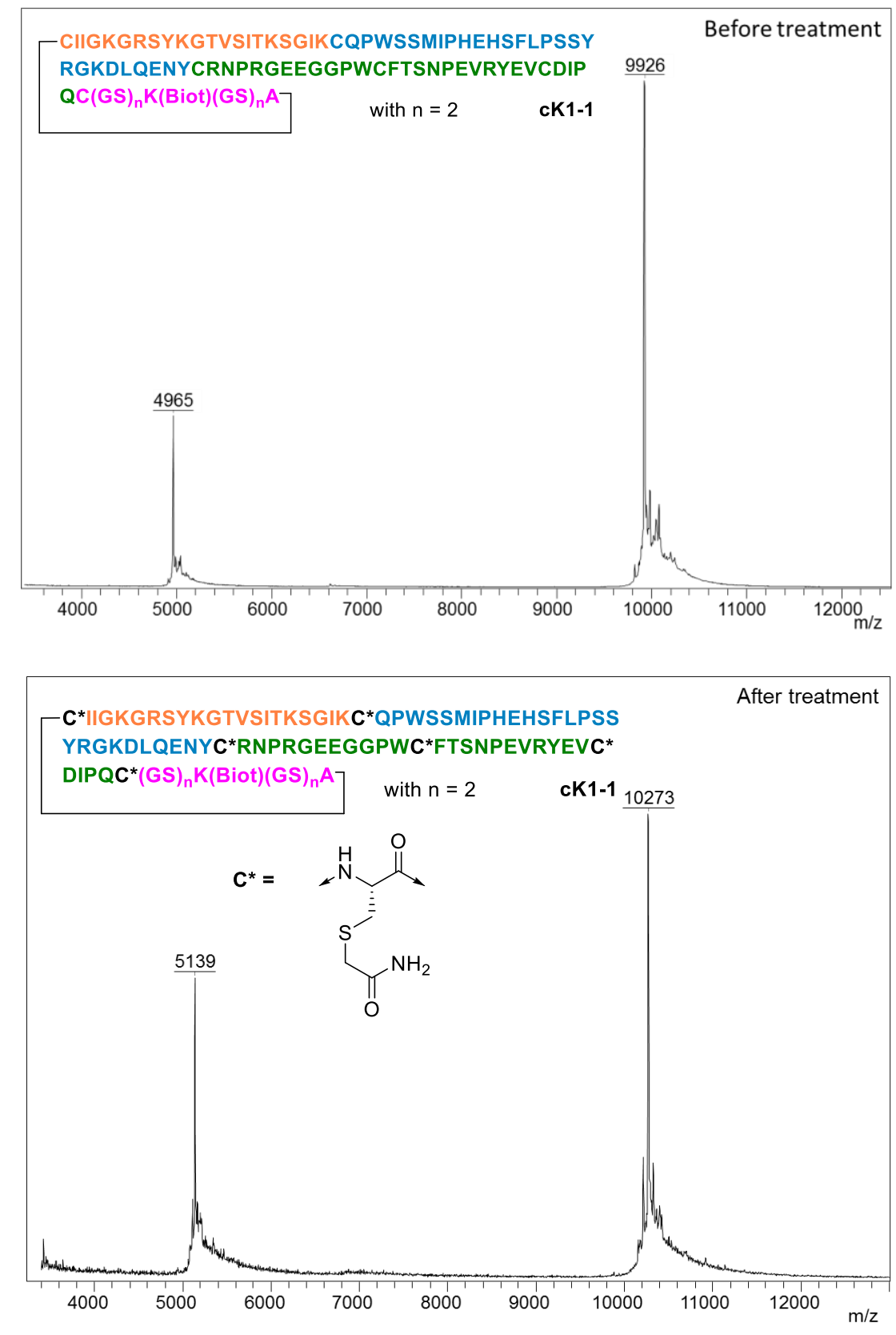

Figure S 117. Monitoring of the cK1-1 alkylation step by MALDI-TOF mass spectrometry (matrix: 2,5-dihydroxybenzoic acid).

\section{Trypsin cleavage}

Trypsin $(0.1 \mathrm{mg} / \mathrm{mL}, 0.5 \mu \mathrm{L})$ was added to the mixture to cleave the alkylated cyclic peptide. The fragments resulting from the enzymatic cleavage were identified by MALDI-TOF MS (Figure S 118a) and the MS-MS sequencing of the ion at m/z 2756.36 confirmed the Gln-Cys and Ala-Cys junctions formed during the cyclisation process (Figure S 118b). 
a)

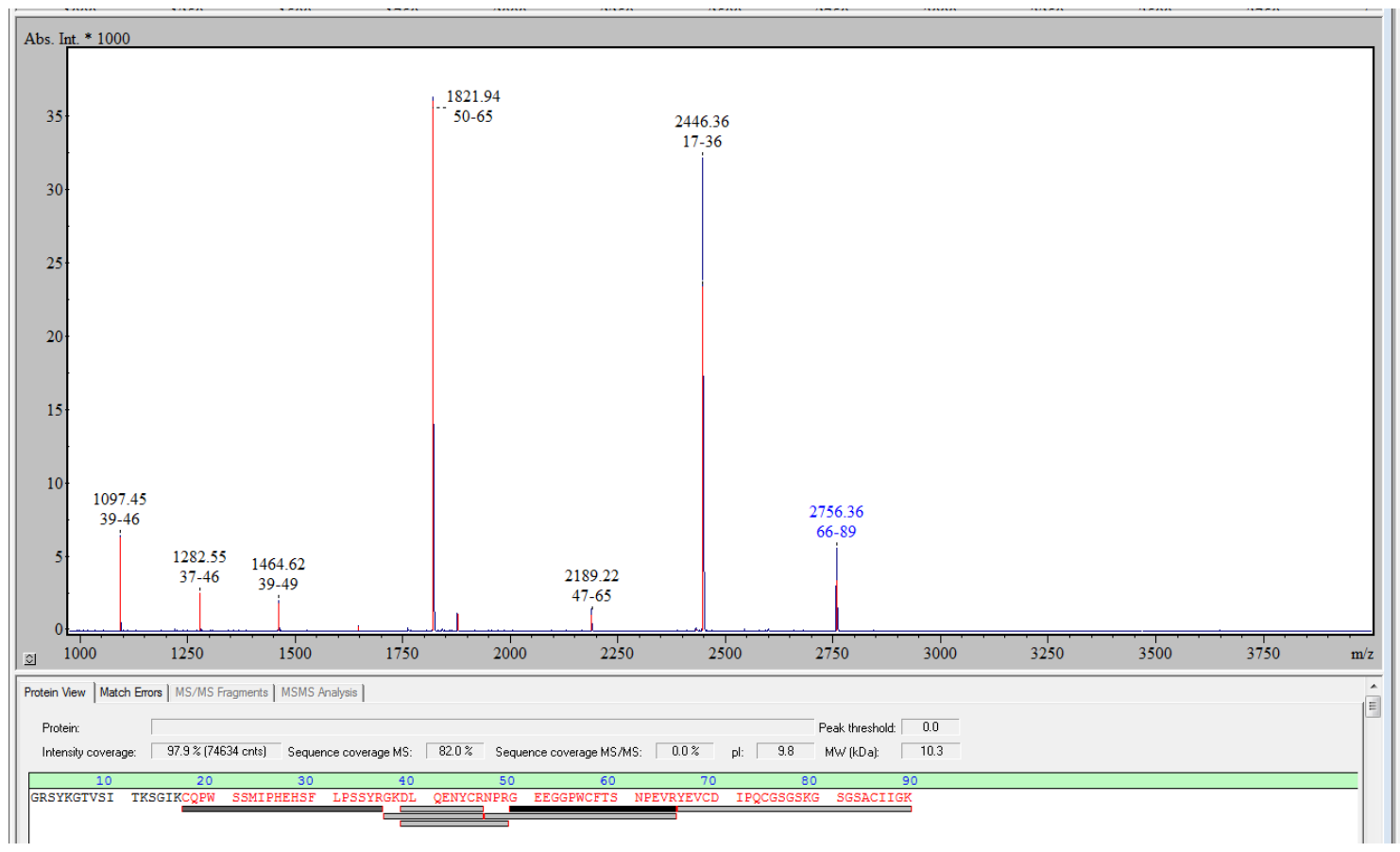

b)

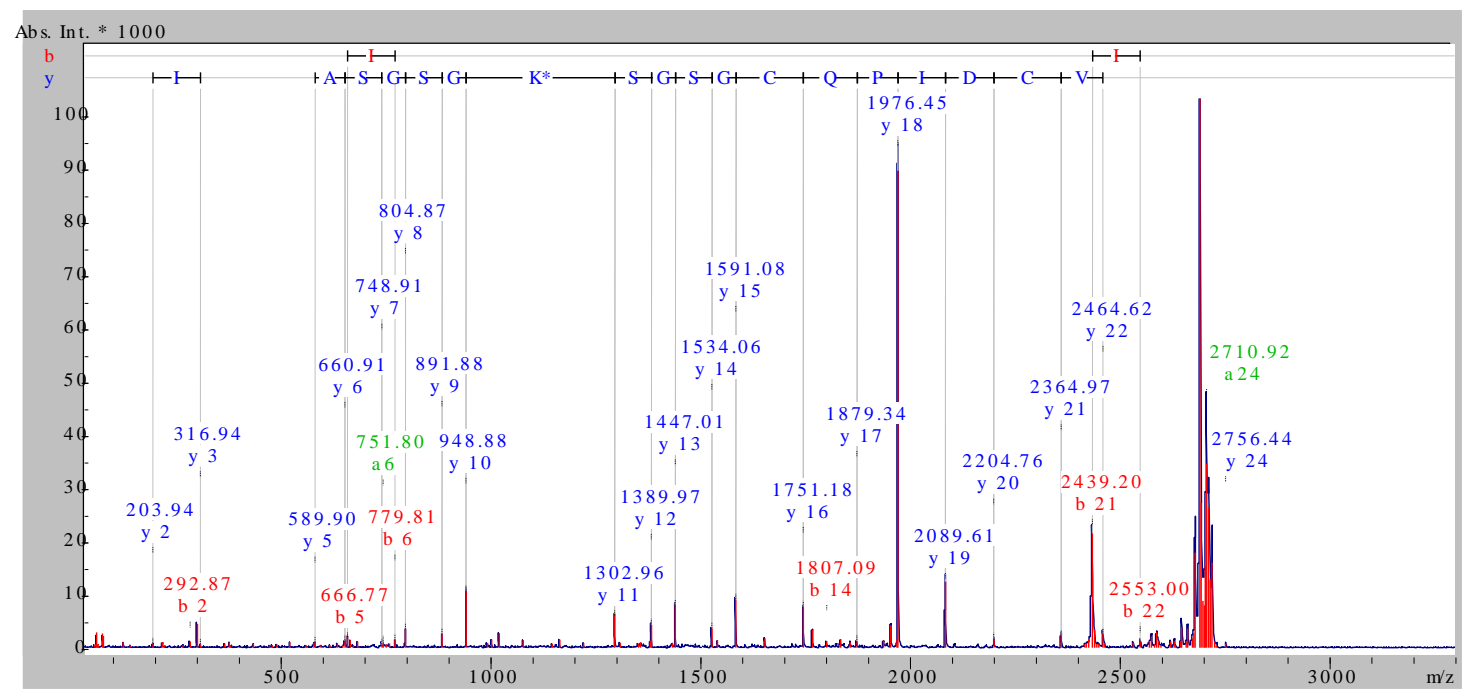

Figure S 118. MALDI-TOF TOF sequencing of cyclic peptide cK1-1 after alkylation and trypsin cleavage using 2,5-dihydroxybenzoic acid as matrix (positive reflector mode). a) MS analysis of the enzymatic lysate (after $90 \mathrm{~min}$ ). b) MS-MS sequencing of the ion at $\mathrm{m} / \mathrm{z} 2756.36$. 


\subsection{Synthesis of cK1-2}

To a solution of $\mathrm{Gn} \cdot \mathrm{HCl}(287 \mathrm{mg})$ in $0.1 \mathrm{M}$, pH 7.4 phosphate buffer $(300 \mu \mathrm{L})$ was added MPAA $(16.8 \mathrm{mg})$ and the $\mathrm{pH}$ of the mixture was adjusted to 7.26 by addition of $6 \mathrm{M} \mathrm{NaOH}(31$ $\mu \mathrm{L})$. Peptide $9 \mathrm{~b}$ (5.00 mg, $0.479 \mu \mathrm{mol}, 1 \mathrm{eq})$ and peptide $8 \mathbf{c}(0.822 \mathrm{mg}, 0.479 \mu \mathrm{mol}, 1 \mathrm{eq})$ were successively dissolved in the MPAA solution $(120 \mu \mathrm{L})$ and the mixture was stirred at $37{ }^{\circ} \mathrm{C}$. The progress of the ligation leading to the bifunctional peptide intermediate was followed by HPLC (Figure S 119).

After completion of the NCL ligation $(3.5 \mathrm{~h})$, the second step of the process leading to the cyclisation of the peptide was induced by addition of TCEP in the reaction mixture. To a solution of $\mathrm{Gn} \cdot \mathrm{HCl}(1.72 \mathrm{~g})$ in $0.1 \mathrm{M}, \mathrm{pH} 7.4$ phosphate buffer $(1.80 \mathrm{~mL})$ were added TCEP.HCl (90.1 mg), sodium ascorbate $(62.4 \mathrm{mg})$ and MPAA $(100.8 \mathrm{mg})$ and the $\mathrm{pH}$ of the mixture was adjusted to 5.52 by addition of $6 \mathrm{M} \mathrm{NaOH}(260 \mu \mathrm{L})$. The previous ligation mixture containing the bifunctional peptide intermediate was then diluted with this solution of TCEP $(2.28 \mathrm{~mL})$ and the reaction mixture was stirred at $37{ }^{\circ} \mathrm{C}$ for $40 \mathrm{~h}$. After completion of the reaction, the mixture was acidified with $\mathrm{AcOH}(0.30 \mathrm{~mL})$ and extracted with $\mathrm{Et}_{2} \mathrm{O}(5 \times)$ to remove the MPAA. The crude was further diluted with water $(9 \mathrm{~mL})$ and purified by HPLC (eluent $\mathrm{A}=$ water containing $0.1 \%$ TFA, eluent $\mathrm{B}=$ acetonitrile in water $4 / 1$ containing $0.1 \%$ TFA, $50{ }^{\circ} \mathrm{C}$, detection at $215 \mathrm{~nm}, 6 \mathrm{~mL} / \mathrm{min}, 0-20 \%$ eluent $\mathrm{B}$ in $5 \mathrm{~min}$, then $20-45 \%$ eluent $\mathrm{B}$ in 60 min, C18XBridge column) to give the cyclic peptide cK1-2 as a white solid after lyophilization $(2.04 \mathrm{mg}, 37 \%)$. 

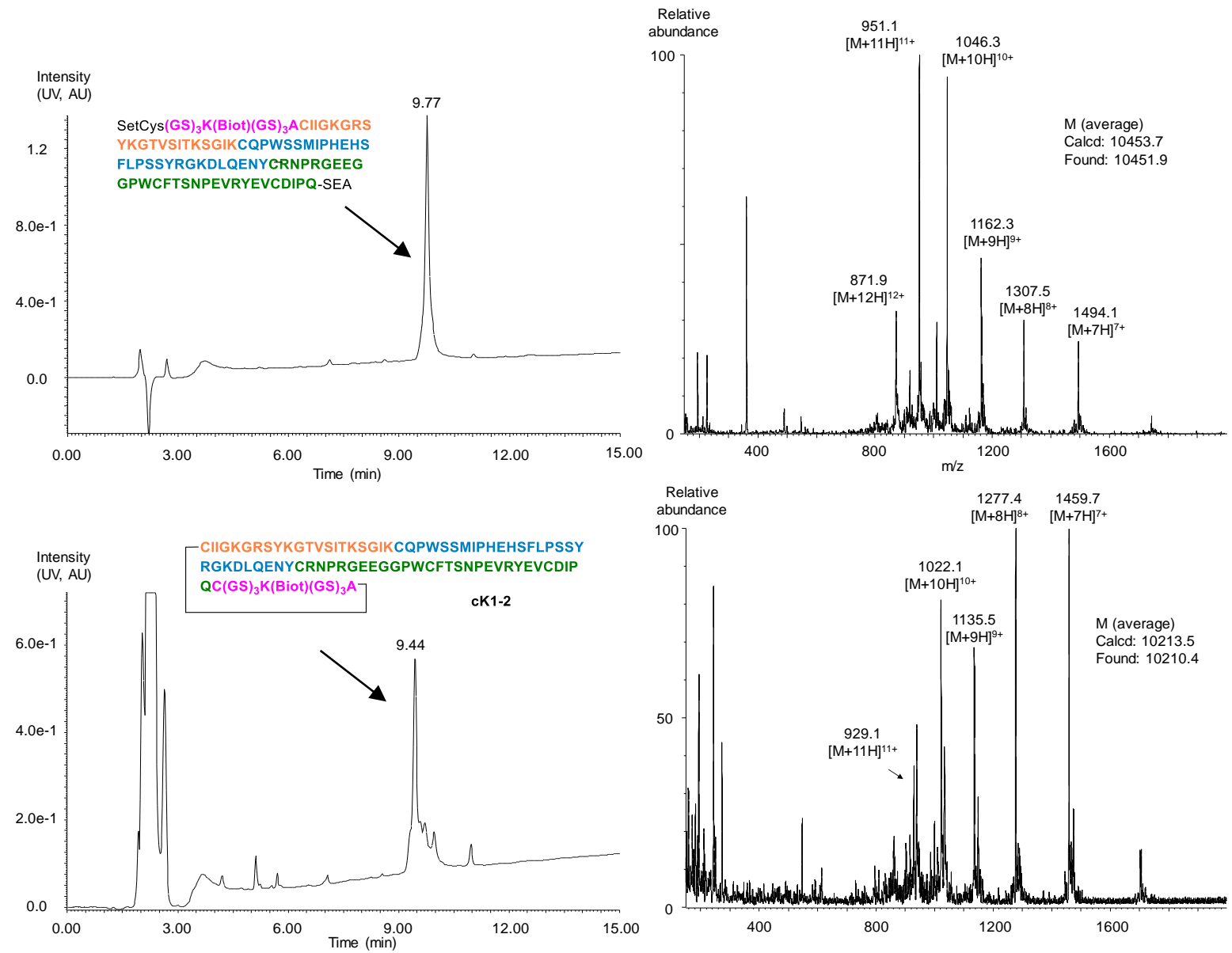

Figure S 119. Assembly of cK1-2 monitored by LC-MS. a) Analysis of the reaction mixture 120 min after mixing the two partners of the ligation reaction. b) Analysis of the reaction mixture $16 \mathrm{~h}$ after the addition of TCEP. LC trace: eluent A $0.10 \%$ TFA in water, eluent B $0.10 \%$ TFA in $\mathrm{CH}_{3} \mathrm{CN} /$ water: $4 / 1$ by vol., $\mathrm{C} 18$ column, gradient $0-100 \% \mathrm{~B}$ in $30 \mathrm{~min}, 50{ }^{\circ} \mathrm{C}, 1$ $\mathrm{mL} / \mathrm{min}$, UV detection at $215 \mathrm{~nm}$. 
a)

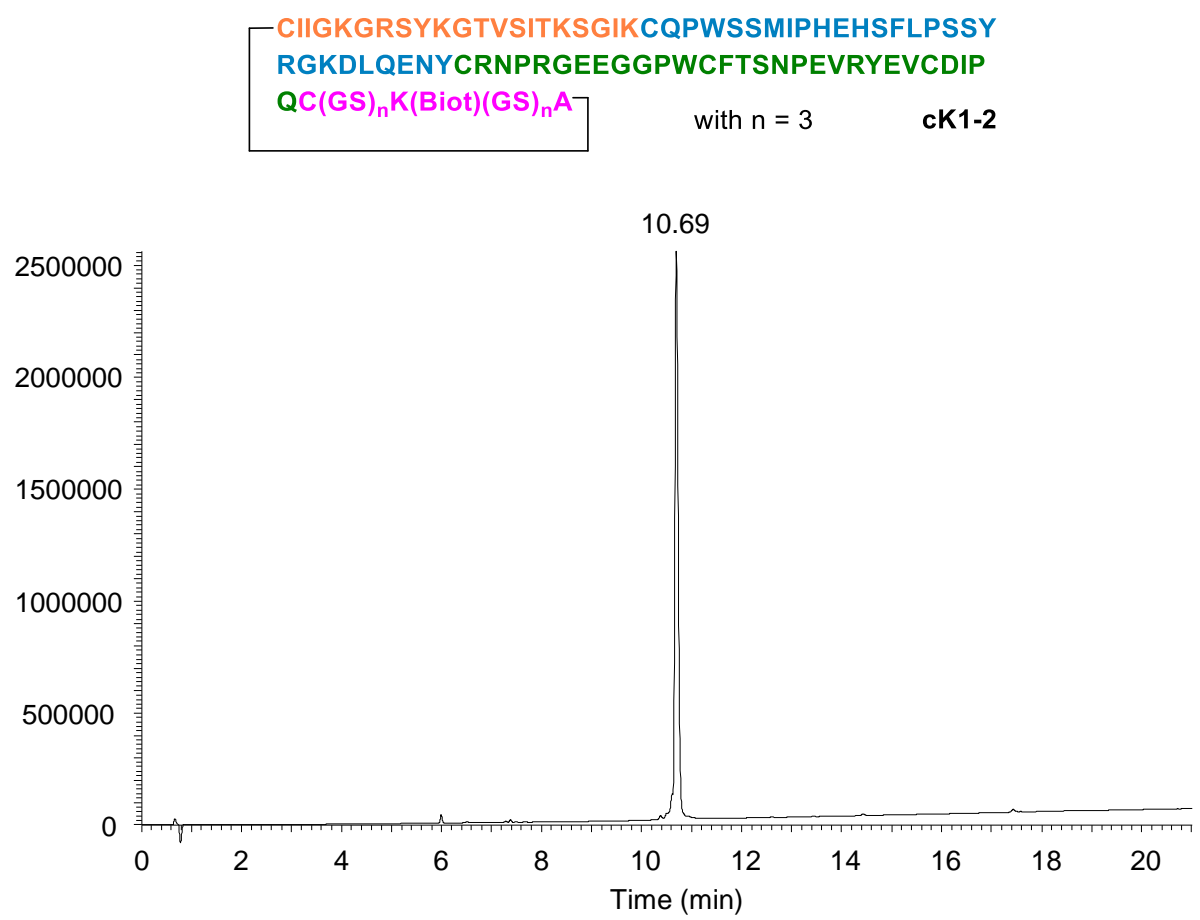

b)

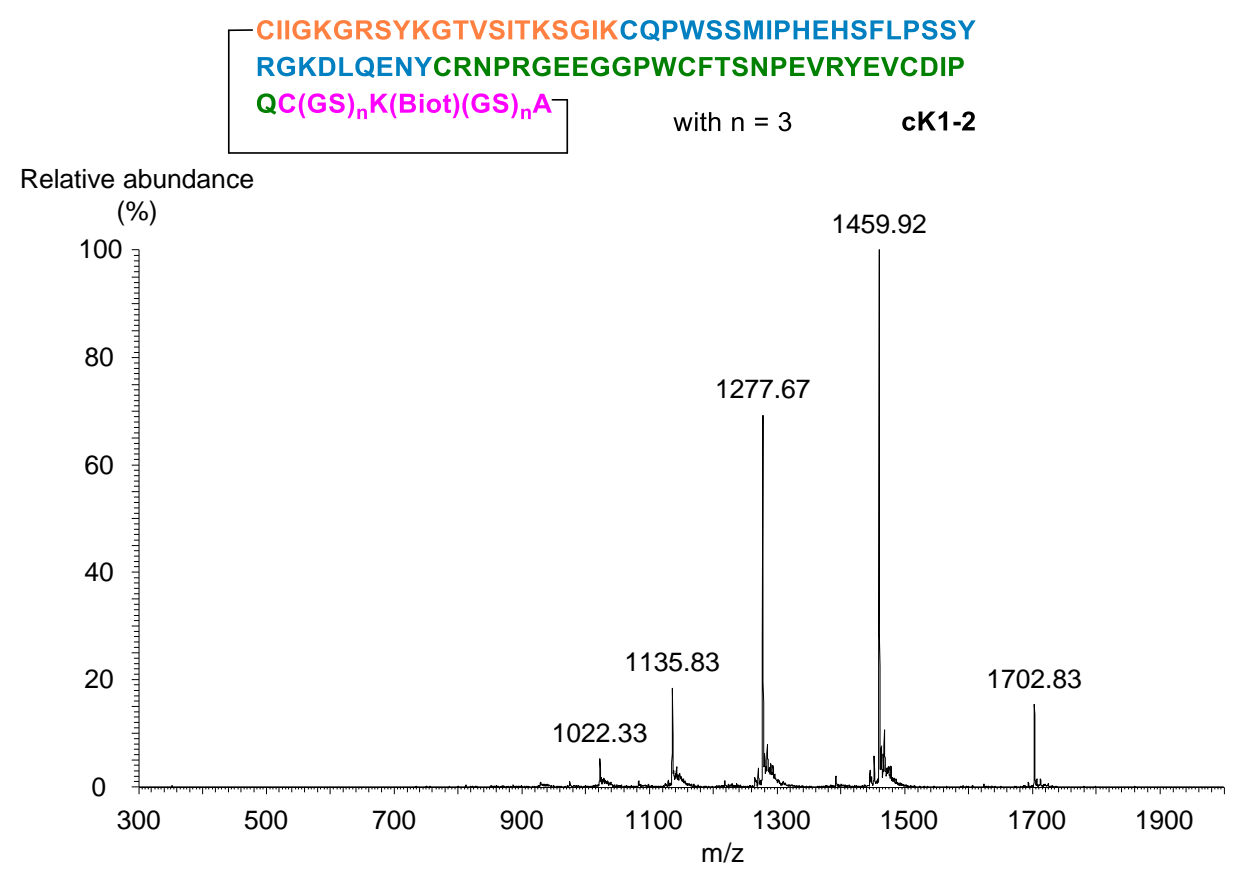

Figure S 120. LC-MS analysis of cK1-2. a) LC trace. Eluent A $0.10 \%$ TFA in water, eluent B $0.10 \%$ TFA in $\mathrm{CH}_{3} \mathrm{CN}$, ACQUITY UPLC Peptide BEH $300 \AA 1.7 \mu \mathrm{m}(2.1 \times 150 \mathrm{~mm})$ column, $50{ }^{\circ} \mathrm{C}$, gradient $0-70 \% \mathrm{~B}$ in $20 \mathrm{~min}, 0.4 \mathrm{~mL} / \mathrm{min}$, UV detection. b) MS trace: $\mathrm{m} / z=1702.83$ $\left([\mathrm{M}+6 \mathrm{H}]^{6+}\right), \quad 1459.92\left([\mathrm{M}+7 \mathrm{H}]^{7+}\right), \quad 1277.67\left([\mathrm{M}+8 \mathrm{H}]^{8+}\right), \quad 1135.83\left([\mathrm{M}+9 \mathrm{H}]^{9+}\right), \quad 1022.33$ $\left([\mathrm{M}+10 \mathrm{H}]^{10+}\right)$; calcd. for M: 10213.45 , found: 10212.71 . 


\subsubsection{Characterization of the cyclic backbone structure of cK1-2}

\section{Reduction-alkylation}

cK1-2 $(20 \mu \mathrm{g})$ was dissolved in a solution of dithiothreitol in $0.025 \mathrm{M}$ ammonium bicarbonate $(1 \mathrm{mg} / \mathrm{mL}, 20 \mu \mathrm{L})$ and the mixture was treated with iodoacetamide $(10 \mathrm{mg} / \mathrm{mL}$ in $0.025 \mathrm{M}$ ammonium bicarbonate, $20 \mu \mathrm{L}$ ) for $15 \mathrm{~min}$. The alkylation step was monitored by MALDITOF mass spectrometry.
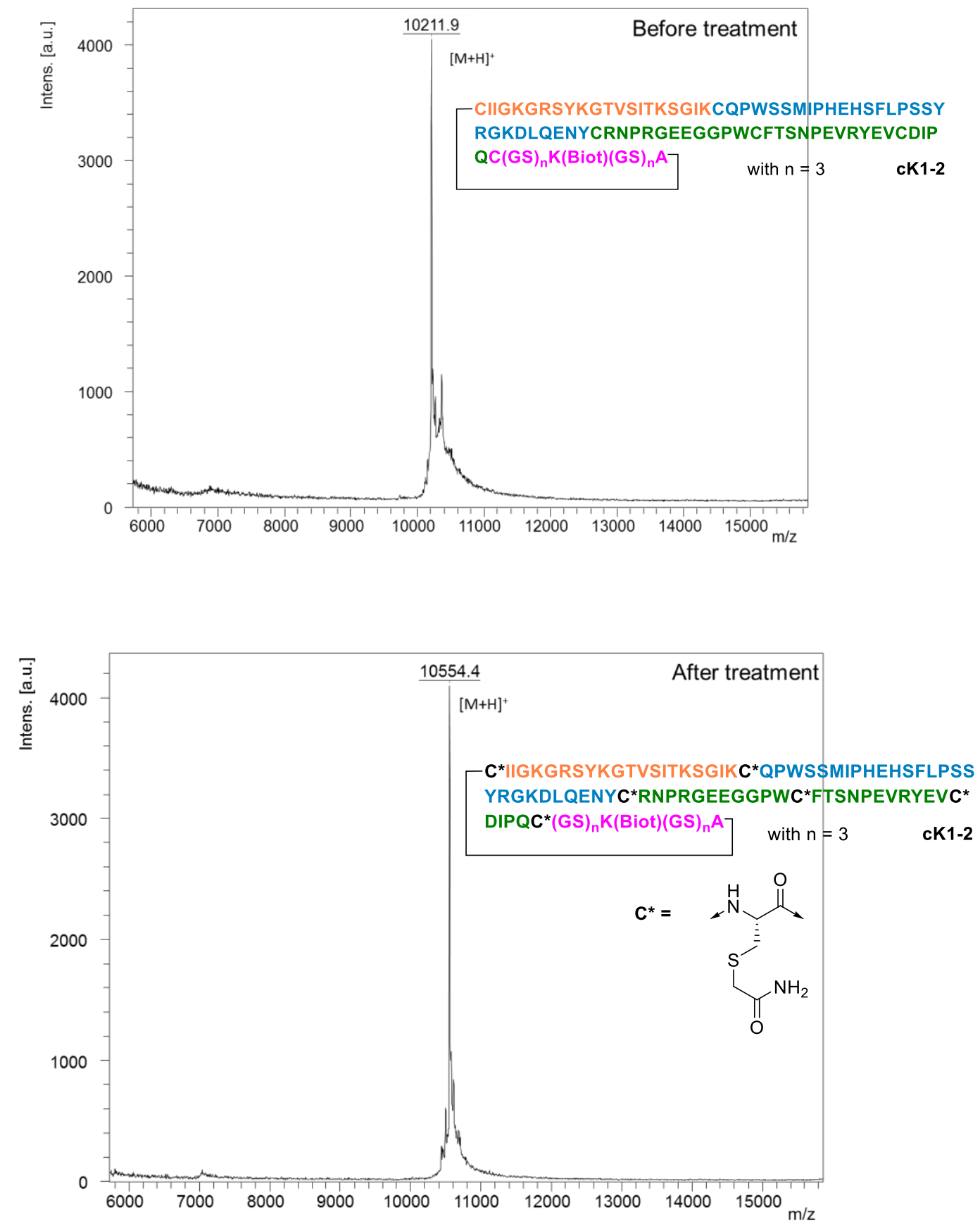

Figure S 121. Monitoring of the cK1-2 alkylation step by MALDI-TOF mass spectrometry (matrix: 2,5-dihydroxybenzoic acid). 


\section{Trypsin cleavage}

Trypsin $(0.1 \mathrm{mg} / \mathrm{mL}, 1 \mu \mathrm{L})$ was added to the mixture to cleave the alkylated cyclic peptide. The fragments resulting from the enzymatic cleavage were identified by MALDI-TOF MS (Figure S 122a) and the MS-MS sequencing of the ion at m/z 3044.05 confirmed the Gln-Cys and Ala-Cys junctions formed during the cyclisation process (Figure S 122b).

a)

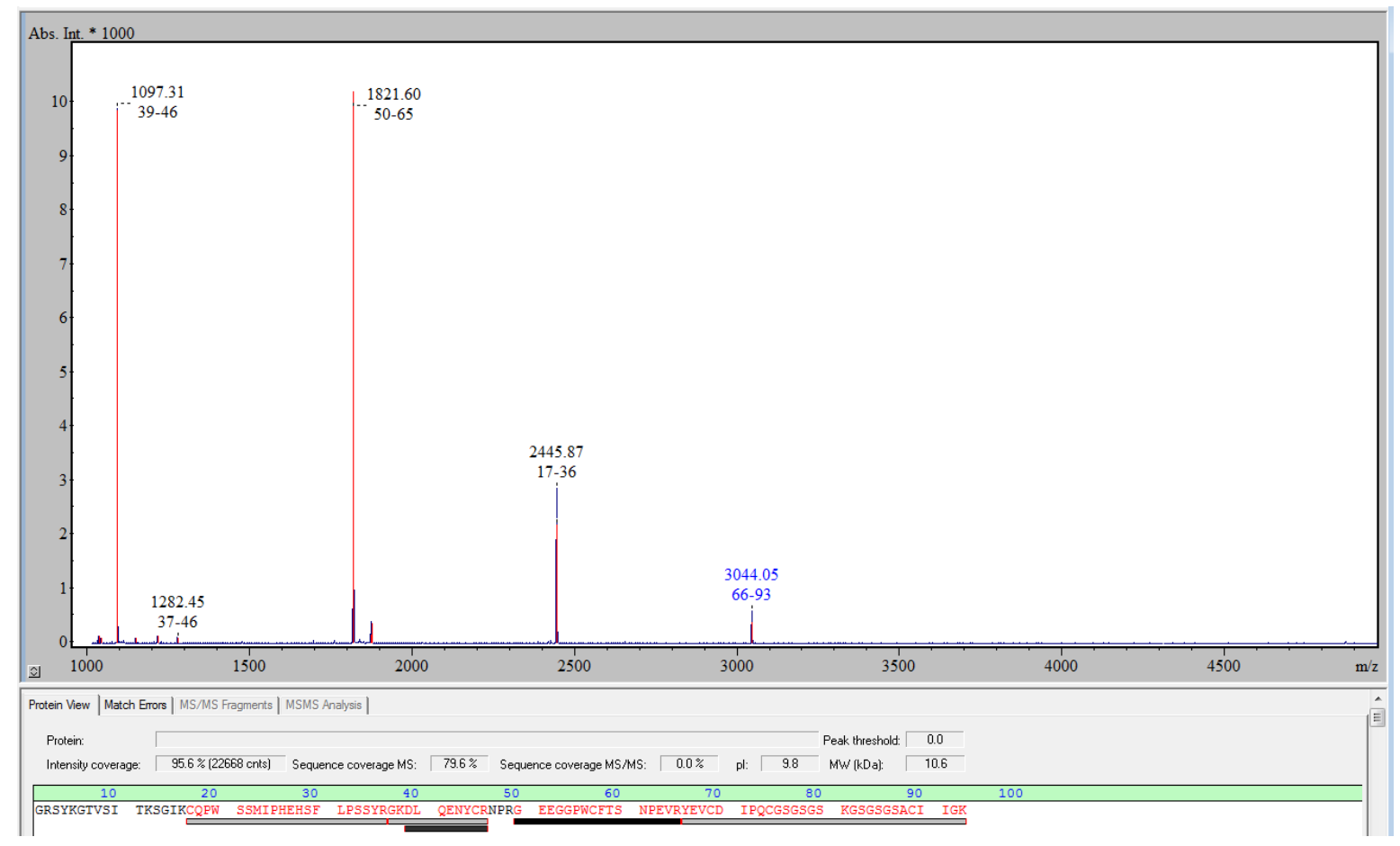

b)

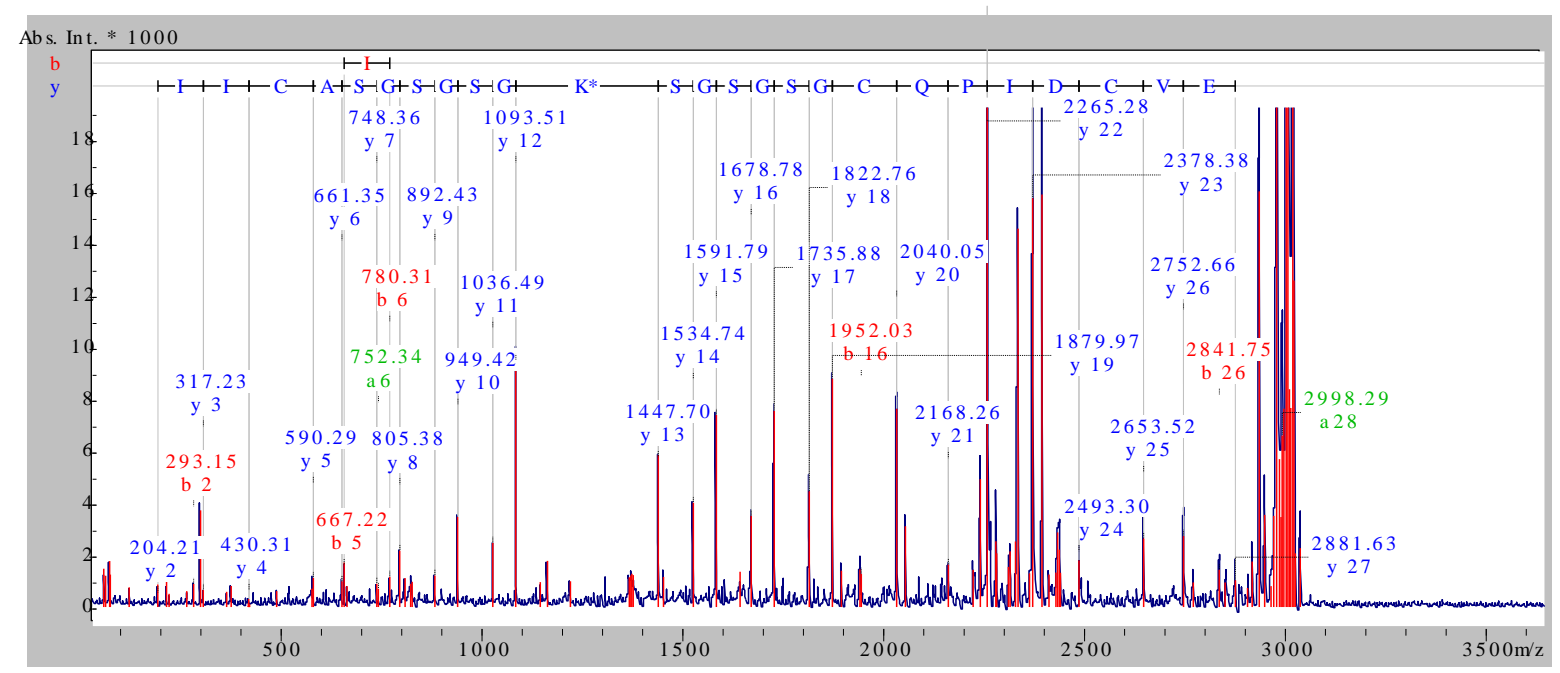

Figure S 122. MALDI-TOF TOF sequencing of cyclic peptide cK1-2 after alkylation and trypsin cleavage using 2,5-dihydroxybenzoic acid as matrix (positive reflector mode). a) MSanalysis of the enzymatic lysate. b) MS-MS sequencing of the ion at m/z 3044.05. 


\section{One-pot synthesis of linear K1 domain (Figure 5a,c)}

\subsection{Preparation of the starting peptide segments}

The sequence of the target K1 polypeptide corresponds to HGF 125-209 using Uniprot numbering of amino acid residues (P14210), with an additional biotinylated lysine residue at the C-terminus.

The SEA peptide segment [125-148]K1-SEA ${ }^{\text {off }}$ was synthesized as described elsewhere. ${ }^{9}$

The biotinylated cysteinyl peptide segment [177-209]K1-NH2 was synthesized as described elsewhere. ${ }^{10}$

Synthesis of the bifunctional peptide SetCys-[150-176]K1-MPA

SetCys-[150-176]K1-SEA peptide was synthesized on $0.05 \mathrm{mmol}$ scale as described in the general procedure. TFA/ $\mathrm{H}_{2} \mathrm{O} / \mathrm{TIS} /$ thiophenol/thioanisol 87.5:2.5:5:2.5:2.5 (8 mL) was used as cleavage cocktail. The $\mathrm{SEA}^{\text {on }}$ peptide which was recovered by precipitation from $\mathrm{Et}_{2} \mathrm{O} /$ heptane was immediately converted into the corresponding MPA thioester using the following procedure.

MPA $(0.625 \mathrm{~mL})$ was dissolved in water $(11.88 \mathrm{~mL})$ and the $\mathrm{pH}$ of the solution was adjusted to 4.0 by addition of $6 \mathrm{M} \mathrm{NaOH}$. The $\mathrm{SEA}^{\text {on }}$ peptide recovered after acidic cleavage was added to this solution of MPA. $\mathrm{Gn} \cdot \mathrm{HCl}(8.58 \mathrm{~g})$ was added until complete dissolution of the peptide. Once the $\mathrm{pH}$ readjusted to 4.0 by addition of $6 \mathrm{M} \mathrm{NaOH}$, the reaction was stirred under inert atmosphere (glovebox) at $37{ }^{\circ} \mathrm{C}$ for $6 \mathrm{~h}$. The reaction mixture was then acidified with $\mathrm{AcOH}$ $(1.5 \mathrm{~mL})$ and extracted with $\mathrm{Et}_{2} \mathrm{O}(5 \times)$ to remove the MPA. Purification of the crude by semipreparative HPLC (eluent A: $0.10 \%$ TFA in water, eluent B: $0.10 \%$ TFA in $\mathrm{CH}_{3} \mathrm{CN}, 20$ $\mathrm{mL} / \mathrm{min}, 0-10 \%$ eluent $\mathrm{B}$ in $5 \mathrm{~min}$ then $10-35 \%$ in $30 \mathrm{~min}, \mathrm{C} 18$ column, detection at $215 \mathrm{~nm}$, $\left.30{ }^{\circ} \mathrm{C}\right)$ provided SetCys-[150-176]K1-MPA peptide as a white solid after lyophilisation (34.7 $\mathrm{mg}, 17 \%)$. 
a)

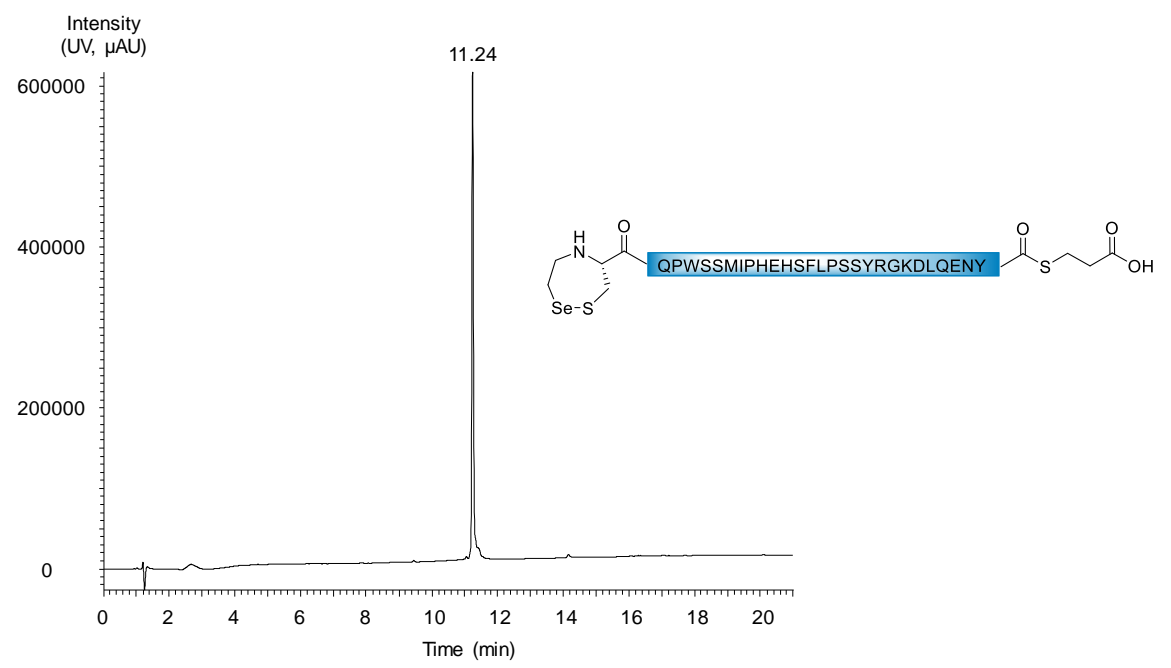

b)

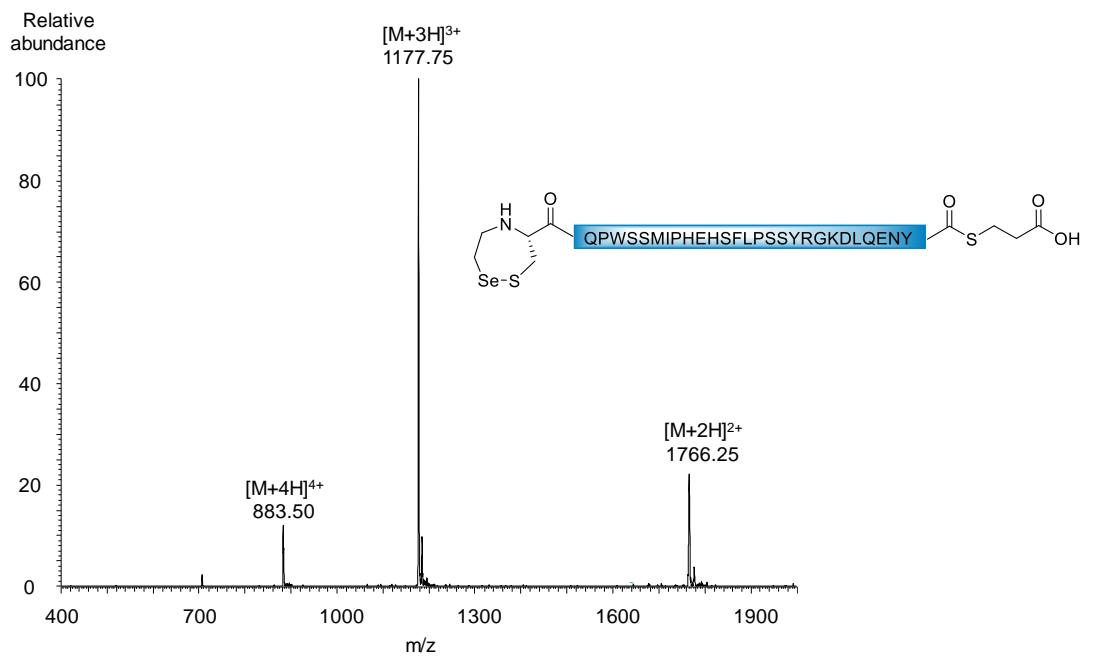

Figure S 123. UPLC-MS analysis of SetCys-[150-176]K1-MPA peptide. a) LC trace. Eluent A: $0.10 \%$ TFA in water, eluent B: $0.10 \%$ TFA in $\mathrm{CH}_{3} \mathrm{CN}$, ACQUITY UPLC Peptide BEH 300 $\AA 1.7 \mu \mathrm{m}(2.1 \times 150 \mathrm{~mm})$ column, $50{ }^{\circ} \mathrm{C}$, gradient $0-70 \% \mathrm{~B}$ in $20 \mathrm{~min}, 0.4 \mathrm{~mL} / \mathrm{min}$, UV detection $(215 \mathrm{~nm}) . \mathrm{b}) \mathrm{MS}$ trace. $\mathrm{m} / \mathrm{z}=1766.25\left([\mathrm{M}+2 \mathrm{H}]^{2+}\right), 1177.75\left([\mathrm{M}+3 \mathrm{H}]^{3+}\right), 883.50$ $\left([\mathrm{M}+4 \mathrm{H}]^{4+}\right)$; calcd. for $\mathrm{M}$ (average): 3530.25 , found: 3530.82 .

\subsection{Method A}

This first one-pot method of synthesis (method A) relies on the sequential addition of the peptide segments (Figure S 124). Elongation of the SetCys peptide segment is first performed by NCL between segments $\mathbf{1 3}$ and $\mathbf{1 4}$ under the mild reductive conditions imposed by the presence of the MPAA catalyst. After completion of the reaction, the SEA peptide segment $\mathbf{1 2}$ is added in the reaction mixture and the second ligation leading to the target $\mathrm{K} 1$ polypeptide is triggered by the concomitant activation of SetCys and the SEA groups with TCEP. 


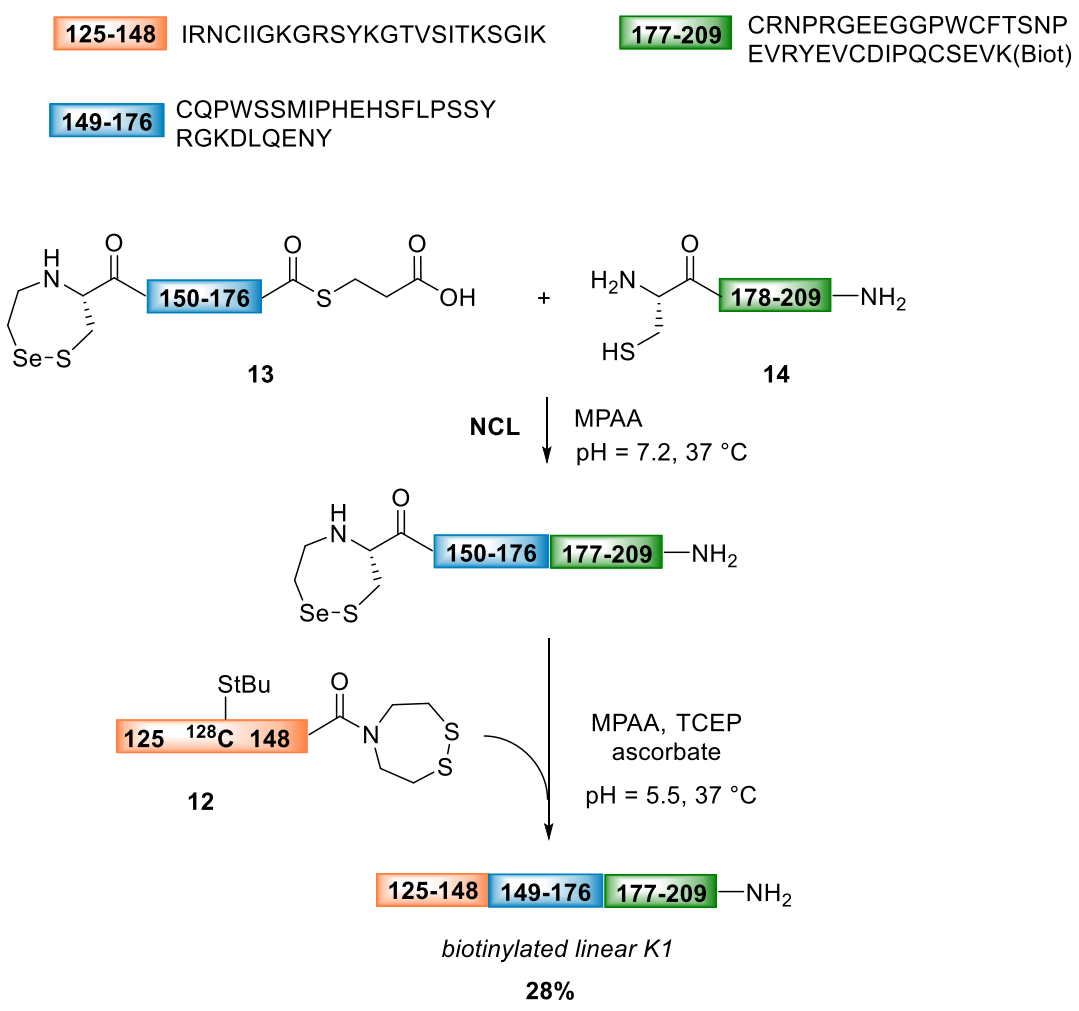

Figure S 124. One-pot three peptide segment assembly of linear K1 polypeptide (method A).

\section{Synthesis of linear K1 using sequential addition of peptides (method A):}

To a solution of $\mathrm{Gn} \cdot \mathrm{HCl}(287 \mathrm{mg})$ in $0.1 \mathrm{M}, \mathrm{pH} 7.4$ phosphate buffer $(300 \mu \mathrm{L})$ was added MPAA $(16.8 \mathrm{mg})$ and the $\mathrm{pH}$ of the mixture was adjusted to 7.22 by addition of $6 \mathrm{M} \mathrm{NaOH}$. Peptide [177-209]K1-NH2 14 (2.78 mg, $0.61 \mu \mathrm{mol}, 1$ eq) and peptide SetCys-[150-176]K1MPA 13 (2.50 mg, $0.61 \mu \mathrm{mol}, 1 \mathrm{eq})$ were successively dissolved in the MPAA solution (152 $\mu \mathrm{L}$ ) and the mixture was stirred at $37^{\circ} \mathrm{C}$. The progress of the ligation leading to the elongated SeCys peptide was followed by UPLC-MS (Figure S 125).

After completion of the NCL ligation $(2.5 \mathrm{~h})$, the second step of the process that finalizes the assembly of the linear peptide K1 was performed by adding the SEA peptide [125-148]K1SEA 12 and TCEP in the reaction mixture. To a solution of $\mathrm{Gn} \cdot \mathrm{HCl}(287 \mathrm{mg})$ in $0.1 \mathrm{M}, \mathrm{pH} 7.4$ phosphate buffer $(300 \mu \mathrm{L})$ were added TCEP. $\mathrm{HCl}(28.6 \mathrm{mg})$, sodium ascorbate $(19.8 \mathrm{mg})$ and MPAA $(16.8 \mathrm{mg}$ ) and the $\mathrm{pH}$ of the mixture was adjusted to 5.23 by addition of $6 \mathrm{M} \mathrm{NaOH}$. The SEA peptide [125-148]K1-SEA was dissolved in the previous ligation mixture containing the elongated SetCys peptide and the resulting mixture was then diluted with the solution of TCEP $(152 \mu \mathrm{L})$. The $\mathrm{pH}$ was readjusted to 5.52 by addition of $6 \mathrm{M} \mathrm{HCl}$ and the mixture was stirred at $37^{\circ} \mathrm{C}$ for $40 \mathrm{~h}$. After completion of the reaction (Figure S 125), the mixture was diluted with $7.5 \% \mathrm{AcOH}$ in water $(8 \mathrm{~mL})$ and extracted with $\mathrm{Et}_{2} \mathrm{O}(5 \times)$ to remove the MPAA. Purification of the crude by HPLC (eluent A: $0.1 \%$ TFA in water, eluent B: $0.1 \%$ TFA in acetonitrile, $6 \mathrm{~mL} / \mathrm{min}, 0-10 \%$ eluent $\mathrm{B}$ in $5 \mathrm{~min}$ then $10-35 \%$ eluent $\mathrm{B}$ in $30 \mathrm{~min}, \mathrm{C} 18$ column, 
$\mathrm{RT}$, detection at $215 \mathrm{~nm}$ ) provided the linear peptide $\mathbf{K} \mathbf{1}$ as a white solid after lyophilization (1.96 mg, 28\%).

a)

a) $\pi: 0.00$ - 2000

b)
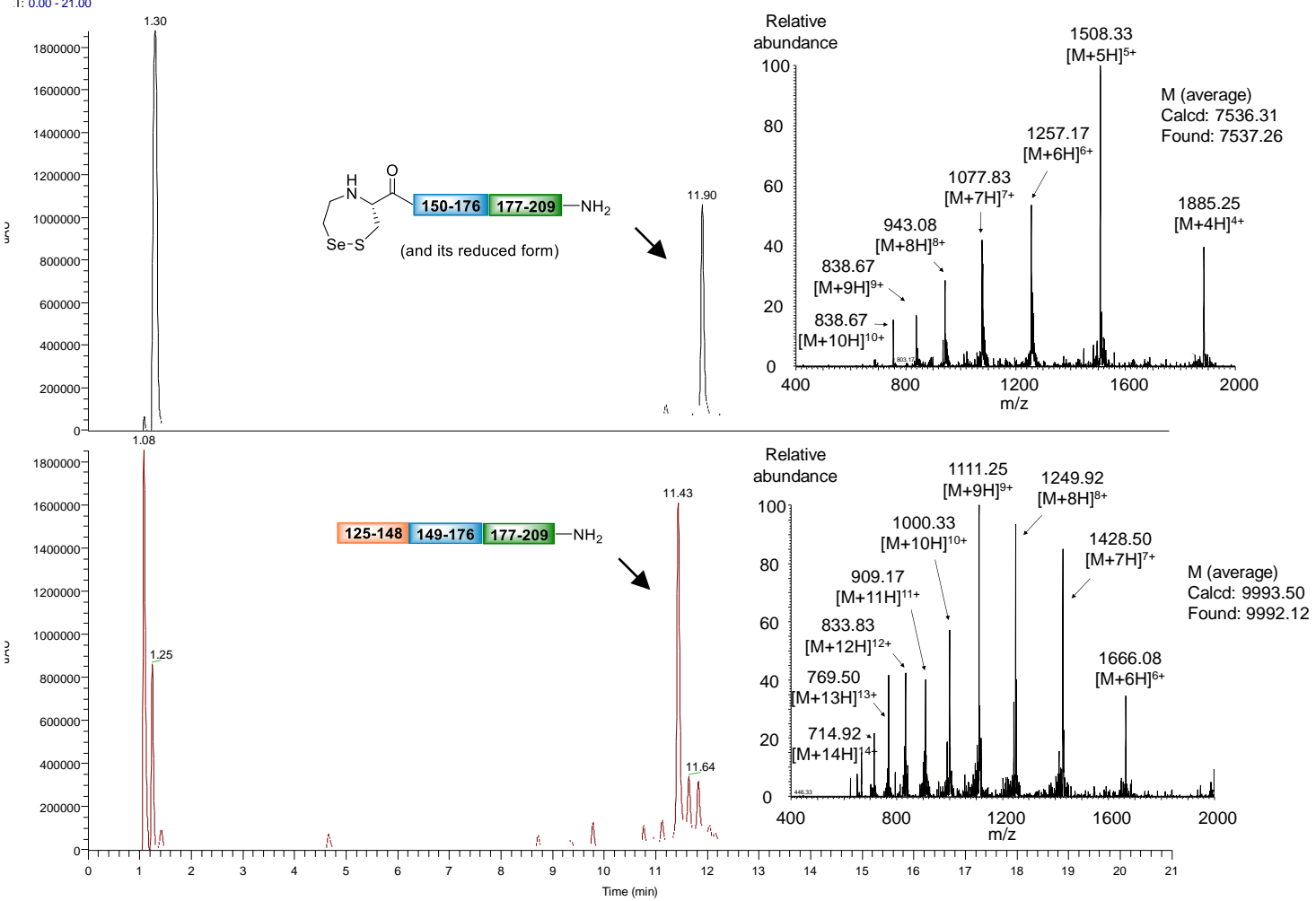

Figure S 125. Assembly of linear K1 polypeptide (method A) monitored by UPLC-MS. a) Analysis of the reaction mixture $16 \mathrm{~h}$ after mixing peptides [177-209] K1- $\mathrm{NH}_{2}$ and SetCys-[150176]K1-MPA. The elongated SetCys peptide was detected in mixture with its reduced form, due to the addition of TCEP in the analyzed sample (to reverse MPAA adducts). b) Analysis of the reaction mixture $48 \mathrm{~h}$ after the addition of the SEA peptide [125-148]K1-SEA and TCEP. LC trace: eluent A $0.10 \%$ TFA in water, eluent B $0.10 \%$ TFA in $\mathrm{CH}_{3} \mathrm{CN}$, ACQUITY UPLC Peptide BEH $300 \AA 1.7 \mu \mathrm{m}(2.1 \times 150 \mathrm{~mm})$ column, $50{ }^{\circ} \mathrm{C}$, gradient $0-70 \% \mathrm{~B}$ in $20 \mathrm{~min}, 0.4$ $\mathrm{mL} / \mathrm{min}$, UV detection.

\subsection{Method B}

This method relies on the latent properties of the SEA group, which enables the SEA peptide segment to be introduced at the beginning of the one-pot process, in mixture with the other reactive peptide segments. A first NCL selectively produces the elongated SetCys peptide, the SEA peptide segment being unreactive under the mild reductive conditions imposed by the MPAA catalyst. The SEA and the elongated SetCys peptide segments were then concomitantly activated by addition of TCEP to produce the final K1 polypeptide. 


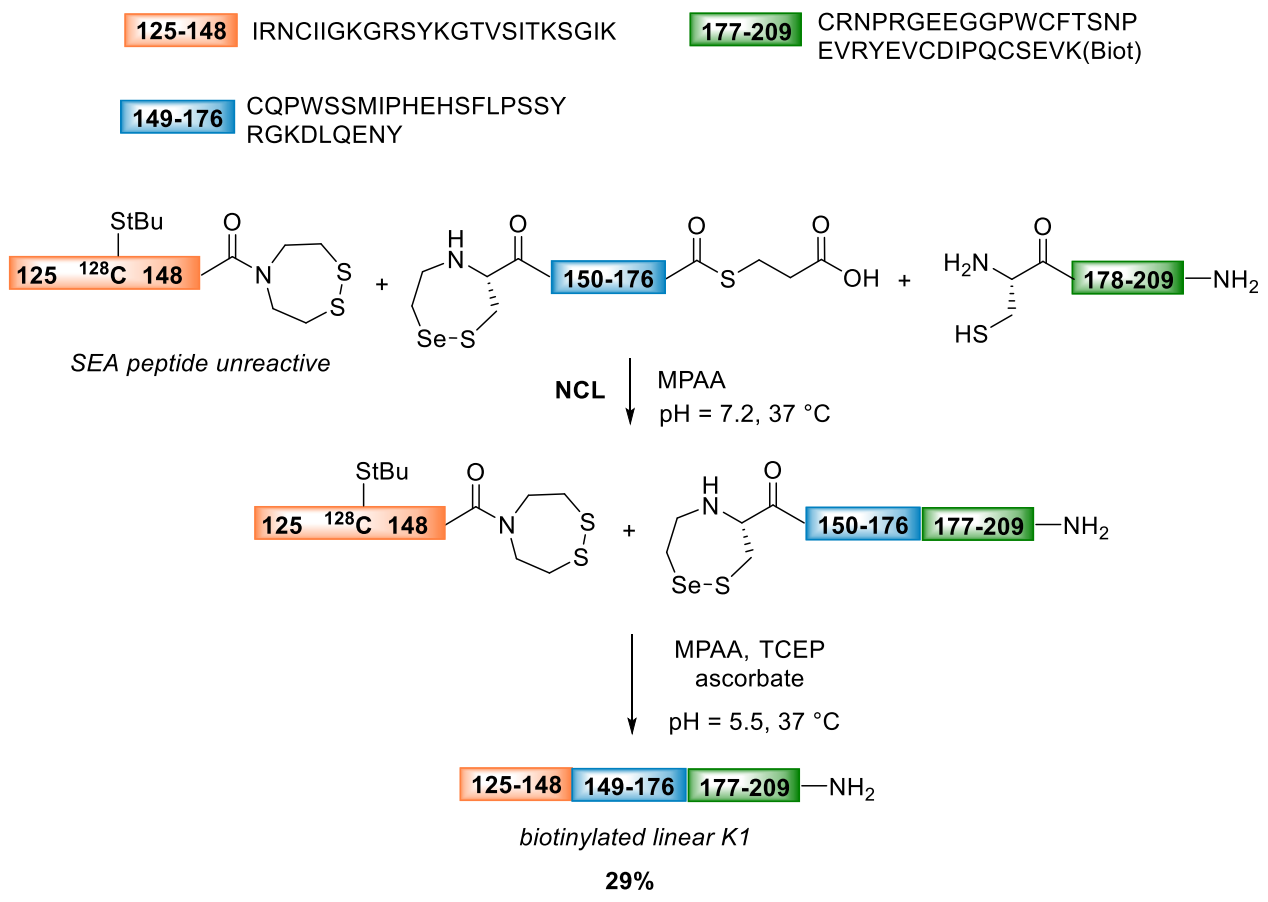

Figure S 126. One-pot three peptide segment assembly of linear K1 polypeptide (method B).

To a solution of $\mathrm{Gn} \cdot \mathrm{HCl}(287 \mathrm{mg})$ in $0.1 \mathrm{M}$, pH 7.4 phosphate buffer $(300 \mu \mathrm{L})$ was added MPAA $(16.8 \mathrm{mg})$ and the $\mathrm{pH}$ of the mixture was adjusted to 7.28 by addition of $6 \mathrm{M} \mathrm{NaOH}$. Peptide [125-148]K1-SEA $(2.18 \mathrm{mg}, 0.61 \mu \mathrm{mol}, 1 \mathrm{eq}),[\mathbf{1 7 7 - 2 0 9}] \mathrm{K} 1-\mathrm{NH}_{2}(2.78 \mathrm{mg}, 0.61 \mu \mathrm{mol}$, 1 eq) and peptide SetCys-[150-176]K1-MPA $(2.50 \mathrm{mg}, 0.61 \mu \mathrm{mol}, 1 \mathrm{eq})$ were successively dissolved in the MPAA solution $(152 \mu \mathrm{L})$ and the mixture was stirred at $37^{\circ} \mathrm{C}$. The progress of the ligation leading to the elongated SeCys peptide was followed by UPLC-MS (Figure S 127a), the SEA peptide being unreactive in these mild reductive conditions.

After completion of the NCL ligation $(2.5 \mathrm{~h})$, the second step of the process that ligates the elongated SetCys peptide with the SEA segment was induced by addition of TCEP. To a solution of $\mathrm{Gn} \cdot \mathrm{HCl}(287 \mathrm{mg})$ in $0.1 \mathrm{M}, \mathrm{pH} 7.4$ phosphate buffer $(300 \mu \mathrm{L})$ were added TCEP. $\mathrm{HCl}(28.6 \mathrm{mg})$, sodium ascorbate $(19.8 \mathrm{mg})$ and MPAA $(16.8 \mathrm{mg})$ and the $\mathrm{pH}$ of the mixture was adjusted to 5.40 by addition of $6 \mathrm{M} \mathrm{NaOH}$. The previous ligation mixture containing the elongated SetCys peptide and SEA segment was then diluted with the solution of TCEP $(152 \mu \mathrm{L})$. The $\mathrm{pH}$ was readjusted to 5.45 by addition of $6 \mathrm{M} \mathrm{HCl}$ and the mixture was stirred at $37^{\circ} \mathrm{C}$ for $40 \mathrm{~h}$. After completion of the reaction (Figure S 127b), the mixture was diluted with $7.5 \% \mathrm{AcOH}$ in water $(8 \mathrm{~mL})$ and extracted with $\mathrm{Et}_{2} \mathrm{O}(5 \times)$ to remove the MPAA. Purification of the crude by HPLC (eluent A: $0.1 \%$ TFA in water, eluent B: $0.1 \%$ TFA in acetonitrile, $6 \mathrm{~mL} / \mathrm{min}, 0-10 \%$ eluent $\mathrm{B}$ in 5 min then 10-35\% eluent B in $30 \mathrm{~min}, \mathrm{C} 18$ column, $\mathrm{RT}$, detection at $215 \mathrm{~nm}$ ) provided the linear peptide $\mathbf{K 1}$ as a white solid after lyophilization (2.05 mg, 29\%). 


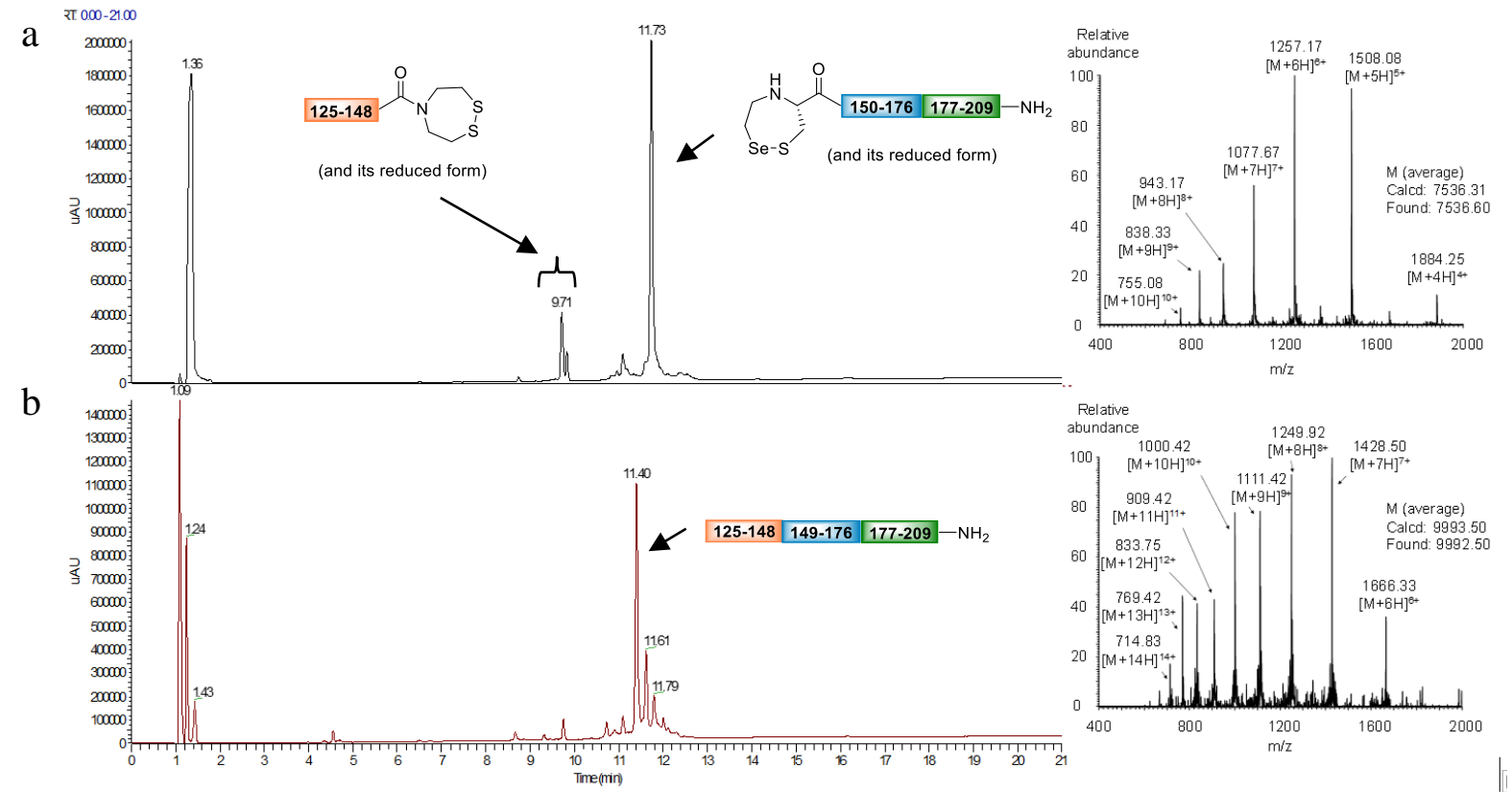

Figure S 127. Assembly of linear K1 polypeptide (method B) monitored by UPLC-MS. a) Analysis of the reaction mixture $2.5 \mathrm{~h}$ after mixing peptides [125-148]K1-SEA, [177-209]K1$\mathrm{NH}_{2}$ and SetCys-[150-176]K1-MPA. The elongated SetCys peptide and the remaining SEA peptide segments are detected in mixture with their reduced form, due to the addition of TCEP in the analyzed sample (to reverse MPAA and StBu adducts). b) Analysis of the reaction mixture $40 \mathrm{~h}$ after the addition of TCEP. LC trace: eluent A $0.10 \%$ TFA in water, eluent $\mathrm{B}$ $0.10 \%$ TFA in $\mathrm{CH}_{3} \mathrm{CN}$, ACQUITY UPLC Peptide BEH $300 \AA 1.7 \mu \mathrm{m}(2.1 \times 150 \mathrm{~mm})$ column, $50{ }^{\circ} \mathrm{C}$, gradient $0-70 \% \mathrm{~B}$ in $20 \mathrm{~min}, 0.4 \mathrm{~mL} / \mathrm{min}$, UV detection. 


\subsection{Characterization of linear K1 polypeptide}

a)

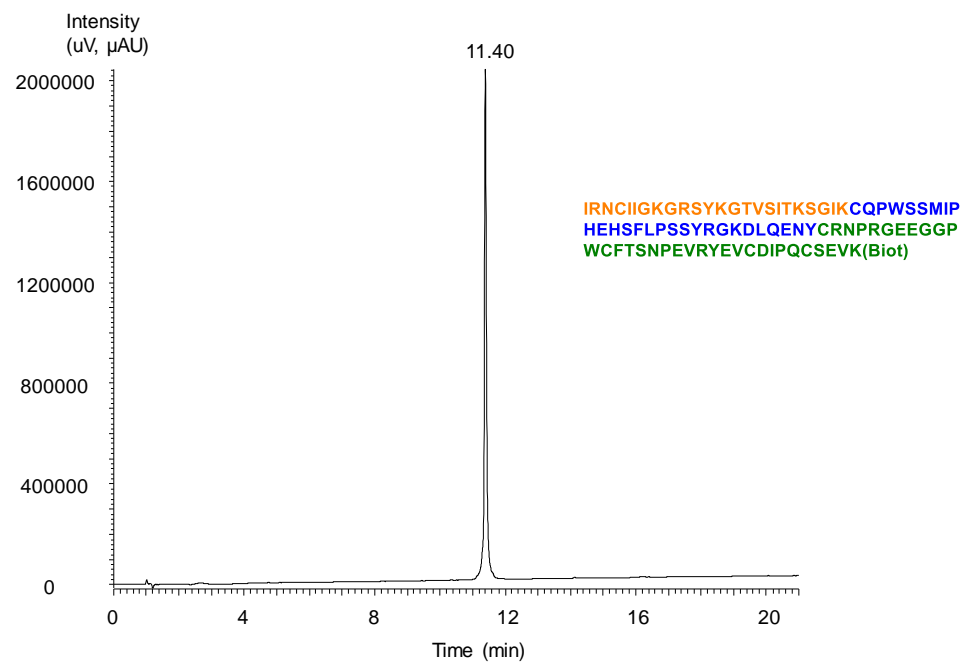

b)

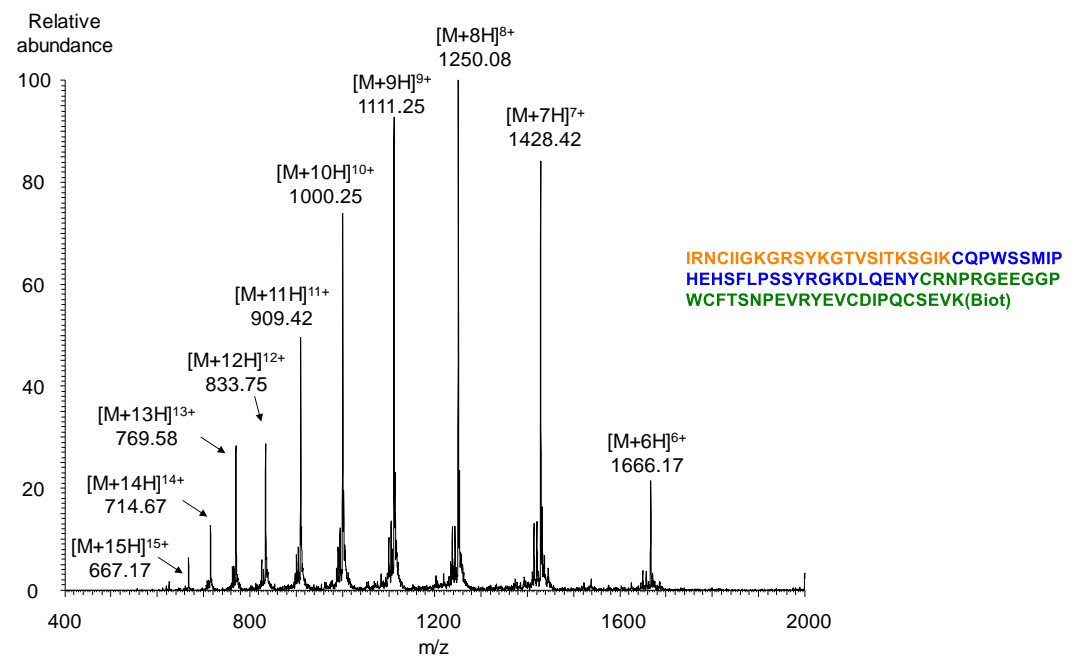

Figure S 128. LC-MS analysis of linear K1 polypeptide. a) LC trace. Eluent A $0.10 \%$ TFA in water, eluent B $0.10 \%$ TFA in $\mathrm{CH}_{3} \mathrm{CN}$, ACQUITY UPLC Peptide BEH $300 \AA 1.7 \mu \mathrm{m}(2.1 \times$ $150 \mathrm{~mm}$ ) column, $50{ }^{\circ} \mathrm{C}$, gradient $0-70 \% \mathrm{~B}$ in $20 \mathrm{~min}, 0.4 \mathrm{~mL} / \mathrm{min}$, UV detection. b) MS trace: $\mathrm{m} / \mathrm{z}=1666.17\left([\mathrm{M}+6 \mathrm{H}]^{6+}\right), 1428.42\left([\mathrm{M}+7 \mathrm{H}]^{7+}\right), 1250.08\left([\mathrm{M}+8 \mathrm{H}]^{8+}\right), 1111.25\left([\mathrm{M}+9 \mathrm{H}]^{9+}\right)$, $1000.25\left([\mathrm{M}+10 \mathrm{H}]^{10+}\right), 909.42\left([\mathrm{M}+11 \mathrm{H}]^{11+}\right), 833.75\left([\mathrm{M}+12 \mathrm{H}]^{12+}\right), 769.58\left([\mathrm{M}+13 \mathrm{H}]^{13+}\right)$, $714.67\left([\mathrm{M}+14 \mathrm{H}]^{14+}\right), 667.17\left([\mathrm{M}+15 \mathrm{H}]^{15+}\right)$. Calcd. for M (average): 9993.40, found: 9992.14 . 


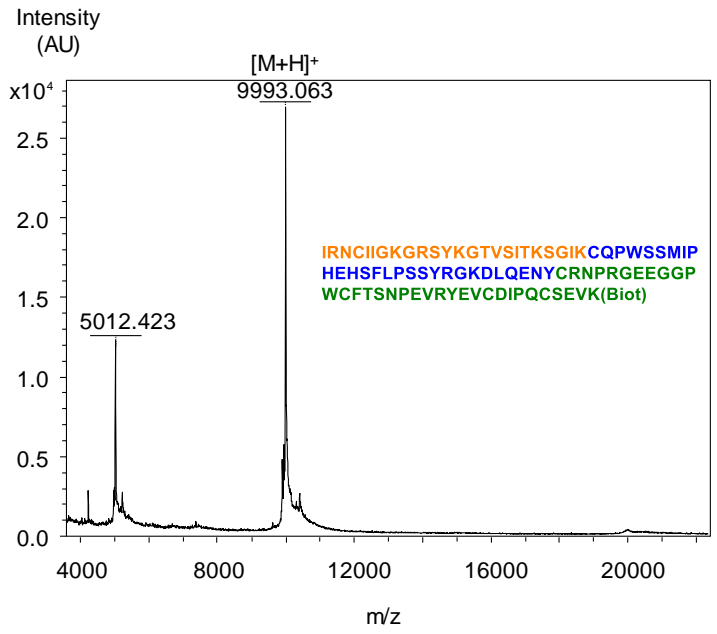

Figure S 129. MALDI-TOF analysis of linear K1 polypeptide. Matrix: 2,5-dihydroxybenzoic acid, positive detection mode, calcd. for $[\mathrm{M}+\mathrm{H}]^{+}$(average): 9994.4, found: 9993.1 .

Proteomic analysis of $\mathrm{K} 1$ polypeptide

\section{Alkylation}

Synthetic K1 polypeptide $(20 \mu \mathrm{g})$ was treated with a solution of iodoacetamide in $0.025 \mathrm{M}$ ammonium bicarbonate $(1 \mathrm{mg} / \mathrm{mL}, 20 \mu \mathrm{L})$ for $15 \mathrm{~min}$. The alkylation step was monitored by MALDI-TOF mass spectrometry. 

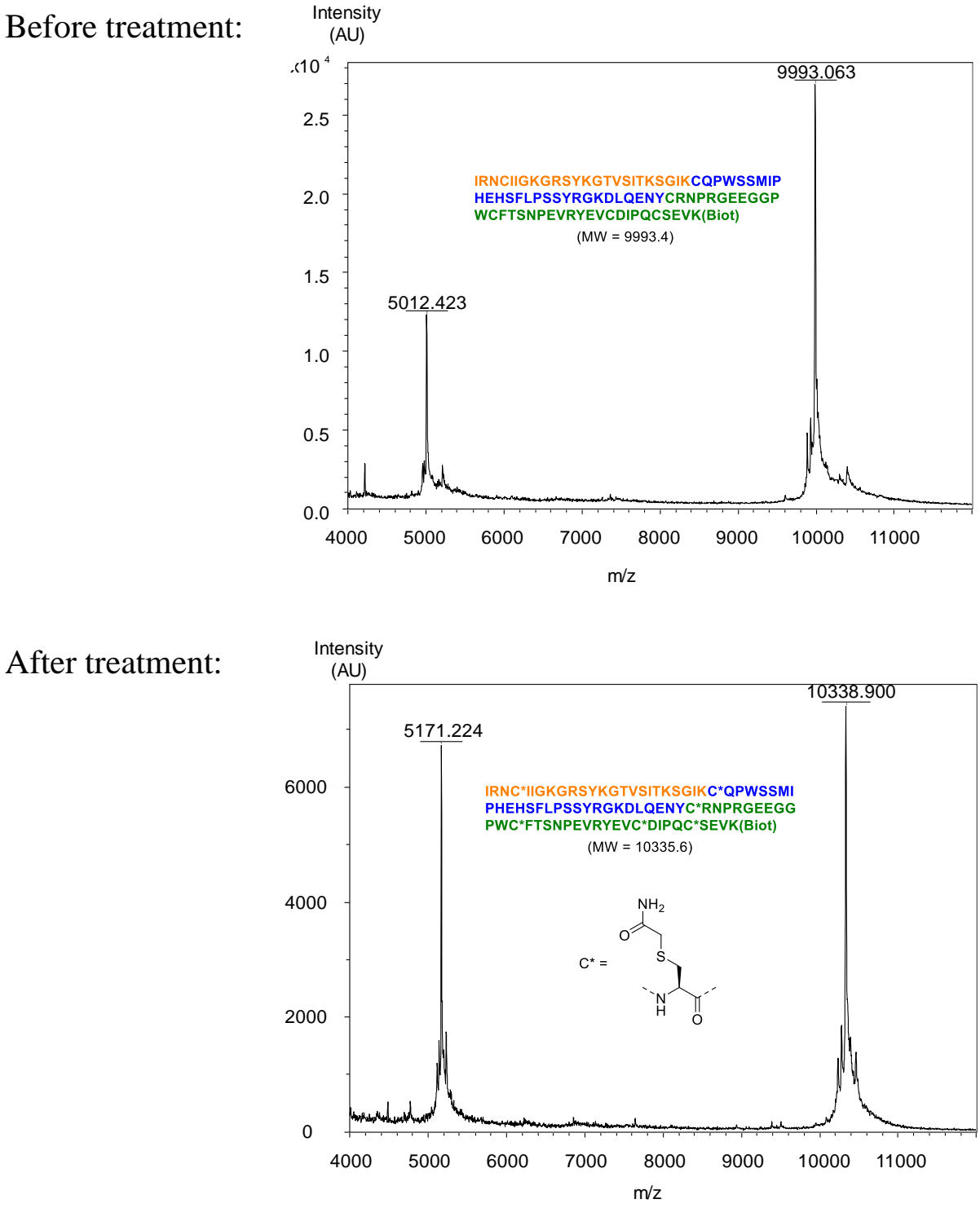

Figure S 130. Monitoring of the linear K1 alkylation step by MALDI-TOF mass spectrometry (matrix: 2,5-dihydroxybenzoic acid). 


\section{Trypsin cleavage}

Trypsin $(0.5 \mathrm{mg} / \mathrm{mL}, 2 \mu \mathrm{L})$ was added to the mixture to cleave the alkylated linear polypeptide. The fragments resulting from the enzymatic cleavage were identified by MALDI-TOF MS.

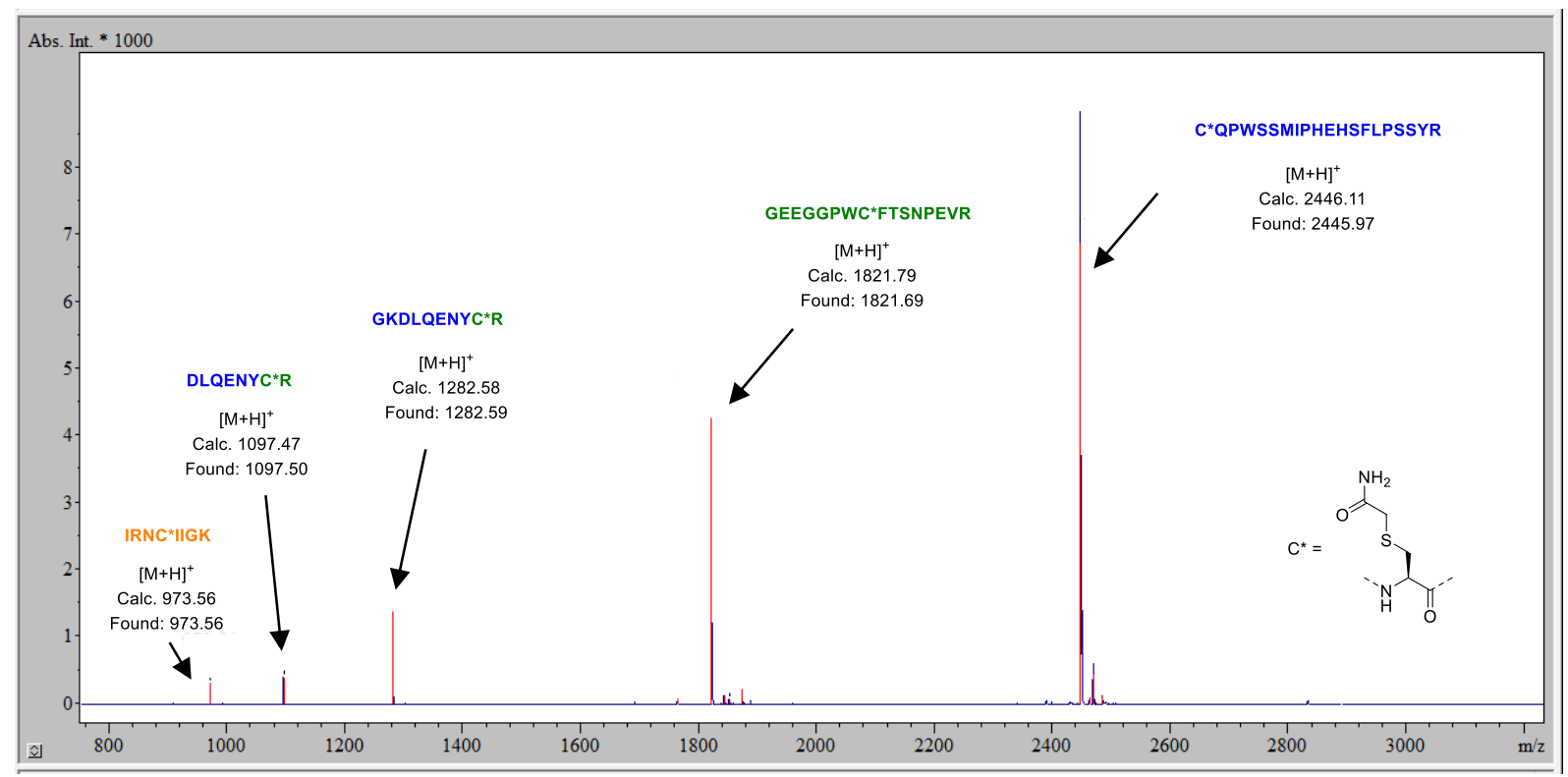

Figure S 131. MALDI-TOF analysis of the enzymatic lysate. Matrix: 2,5-dihydroxybenzoic acid, positive detection mode. Analysis of the fragments produced by enzymatic cleavage of alkylated K1 confirms the Tyr-Cys junction formed by NCL and the conversion of the SetCys residue into Cys residue during the second ligation step. 
10 Folding, characterization and biological activity of cK1-1 and cK1-2 (Figure 6)

\subsection{Folding (Figure 6b,c)}

cK1-1 and cK1-2 were simultaneously folded using a mixture of oxidized and reduced gluthatione.

- $\quad$ K1 cyclized with the short biotinylated linker $(n=2)$

CIIGKGRSYKGTVSITKSGIKCQPWSSMIPHEHSFLPSSYRGKDLQEN
YCRNPRGEEGGPWCFTSNPEVRYEVCDIPQC(GS) ${ }_{2} \mathrm{~K}$ (Biot)(GS) ${ }_{2} \mathrm{~A}$

- $\quad$ K1 cyclized with the long biotinylated linker $(n=3)$

\begin{tabular}{ll} 
CK1-2 & CIIGKGRSYKGTVSITKSGIKCQPWSSMIPHEHSFLPSSYRGKDLQEN \\
YCRNPRGEEGGPWCFTSNPEVRYEVCDIPQC(GS) & K(Biot)(GS) ${ }_{3} \mathrm{~A}$ \\
\hline
\end{tabular}

General procedure:

The cyclic polypeptide cK1-1 or cK1-2 was dissolved in $10 \mathrm{mM} \mathrm{PBS,} 138 \mathrm{mM} \mathrm{NaCl}, 2.7 \mathrm{mM}$ $\mathrm{KCl}$ buffer $\mathrm{pH} 7.4$ containing $10 \%$ by vol of glycerol, $1 \mathrm{mM}$ reduced glutathione, $0.2 \mathrm{mM}$ oxidized glutathione $\left(33 \mathrm{~mL} / \mu \mathrm{mol}\right.$ of peptide). The reaction mixture was gently stirred at $4{ }^{\circ} \mathrm{C}$ for $36 \mathrm{~h}$.

The folding of cK1-1 and cK1-2 was followed by LC-MS. Results are given in Figure S 132Figure S 135.

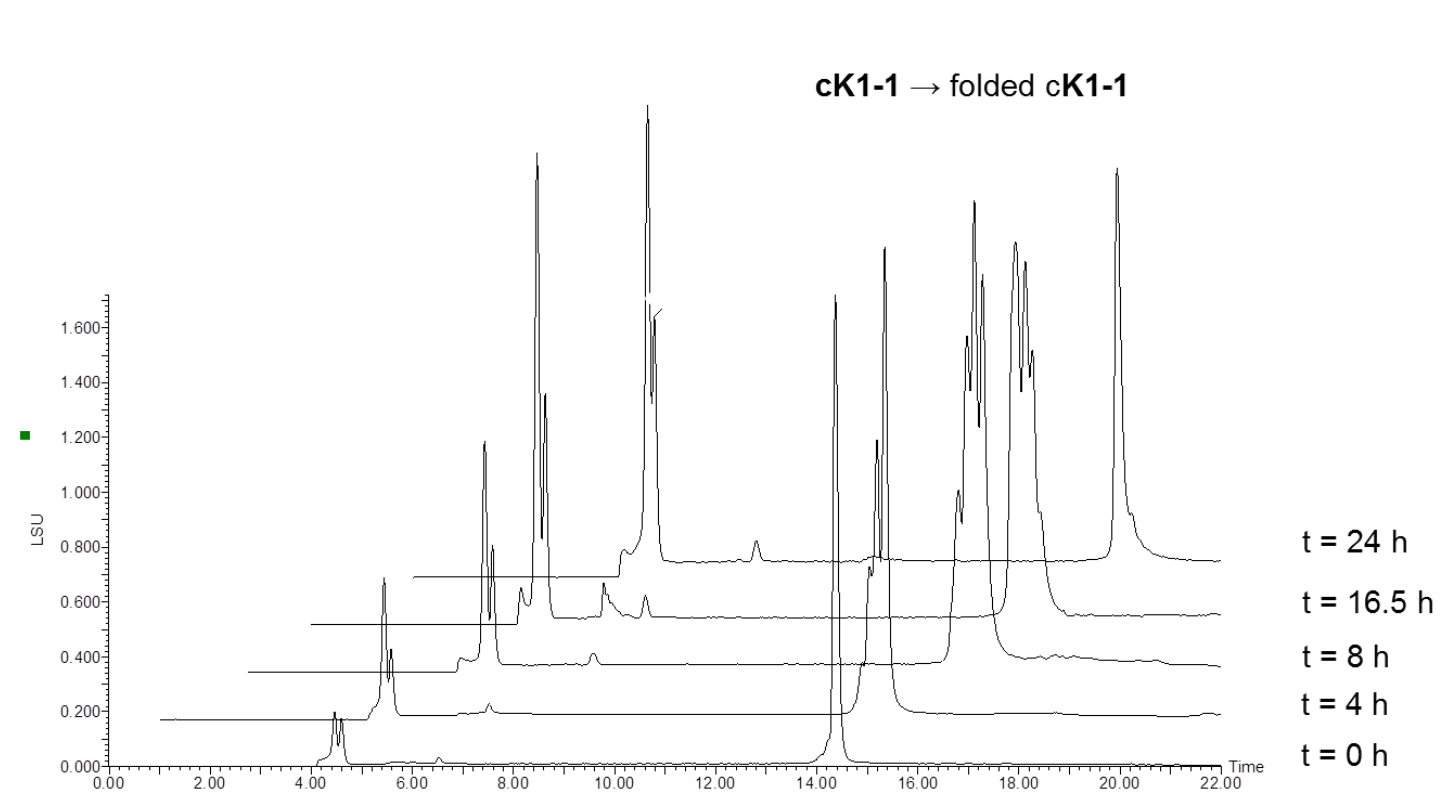

Figure S 132. Kinetics of cK1-1 folding studied by LC-MS. Elution conditions: eluent A $0.10 \%$ TFA in water, eluent B $0.10 \%$ TFA in $\mathrm{CH}_{3} \mathrm{CN} /$ water: $4 / 1$ by vol., XBridge Peptide BHE C18 $3.5 \mu \mathrm{m} 4.6 \times 150 \mathrm{~mm}$ column, gradient $0-50 \% \mathrm{~B}$ in $15 \mathrm{~min}, 50{ }^{\circ} \mathrm{C}, 1 \mathrm{~mL} / \mathrm{min}$, light scattering detection. 


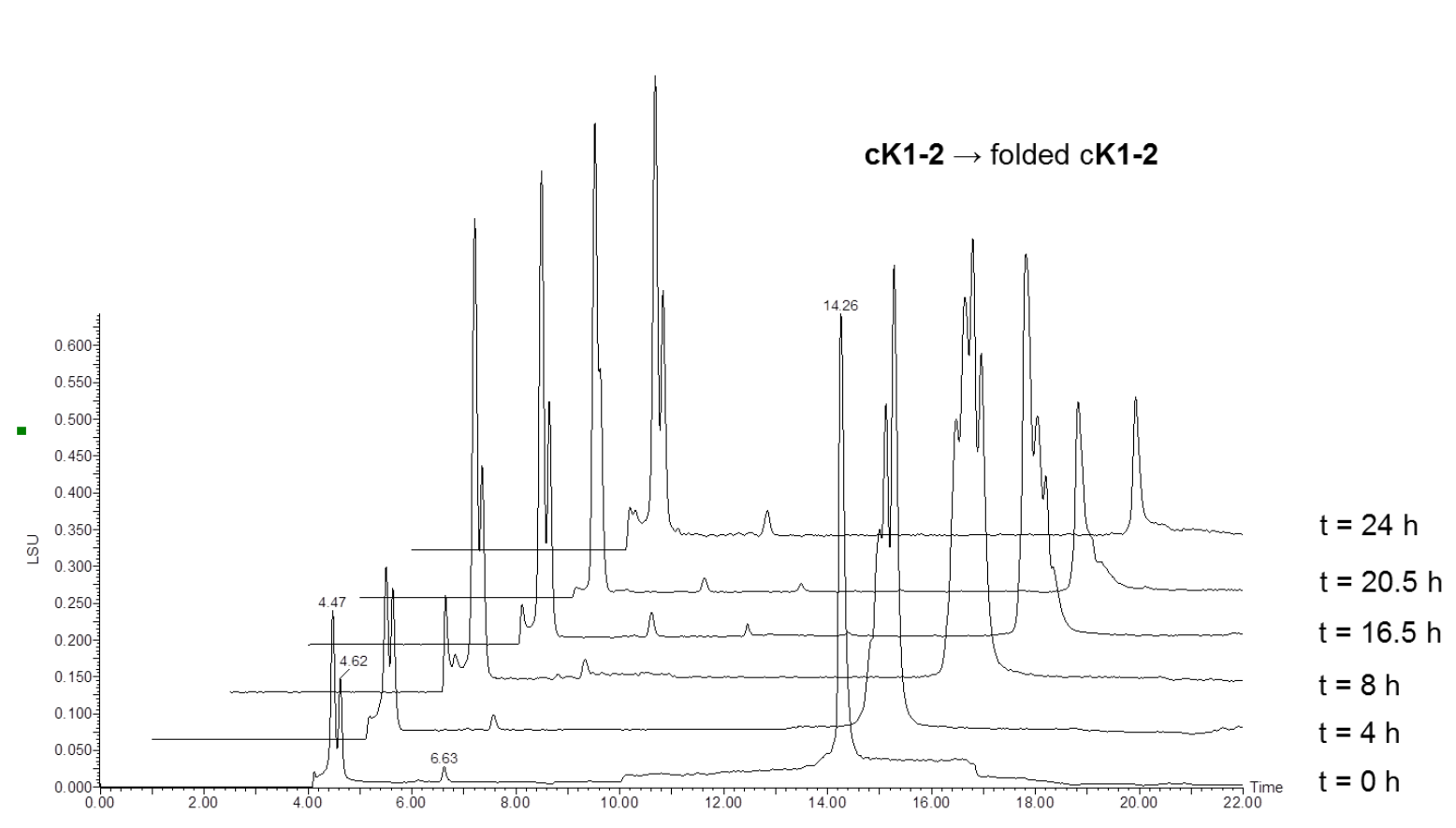

Figure S 133. Kinetics of cK1-2 folding studied by LC-MS. Elution conditions: eluent A $0.10 \%$ TFA in water, eluent B $0.10 \%$ TFA in $\mathrm{CH}_{3} \mathrm{CN} /$ water: $4 / 1$ by vol., XBridge Peptide BHE C18 $3.5 \mu \mathrm{m} 4.6 \times 150 \mathrm{~mm}$ column, gradient $0-50 \% \mathrm{~B}$ in $15 \mathrm{~min}, 50{ }^{\circ} \mathrm{C}, 1 \mathrm{~mL} / \mathrm{min}$, light scattering detection.

The mixtures were then centrifugated $(15300 \times \mathrm{g})$ at $4{ }^{\circ} \mathrm{C}$ during $20 \mathrm{~min}$ to eliminate the insoluble aggregates. The mixtures were transferred in an ultrafiltration column (Vivaspin, 2 $\mathrm{mL})$ with a cut-off of $3000 \mathrm{MWCO}$ and centrifugated $(12000 \times \mathrm{g})$ at $4{ }^{\circ} \mathrm{C}$.

Once concentrated, the proteins were dialysed twice (respectively $1 \mathrm{~h}$ and $18 \mathrm{~h}$ ) at $4{ }^{\circ} \mathrm{C}$ against $10 \mathrm{mM}$ PBS, $138 \mathrm{mM} \mathrm{NaCl}, 2.7 \mathrm{mM} \mathrm{KCl}$ buffer $\mathrm{pH} 7.4$ containing $10 \%$ in vol of glycerol (2 $\times 0.3 \mathrm{~L}$ ). Finally, the concentration of the folded proteins was determined using BCA assay.

Table S 6. Folding results

\begin{tabular}{|ccccc|} 
peptide & $\begin{array}{c}\text { Initial mass of peptide } \\
\text { (in mg) }\end{array}$ & $\begin{array}{c}\text { Volume after } \\
\text { dialys (in } \boldsymbol{\mu L} \text { ) }\end{array}$ & $\begin{array}{c}\text { Concentration of peptide } \\
\text { determined by BCA (in } \boldsymbol{\mu m o l} \text { ) }\end{array}$ & $\begin{array}{c}\text { Yield } \\
\text { (in \%) }\end{array}$ \\
\hline cK1-1 & 1.056 & 312 & 30 & 12 \\
\hline cK1-2 & 1.084 & 230 & 26 & 7 \\
\hline
\end{tabular}




\subsubsection{Characterization of folded cK1-1f and cK1-2f}

a)

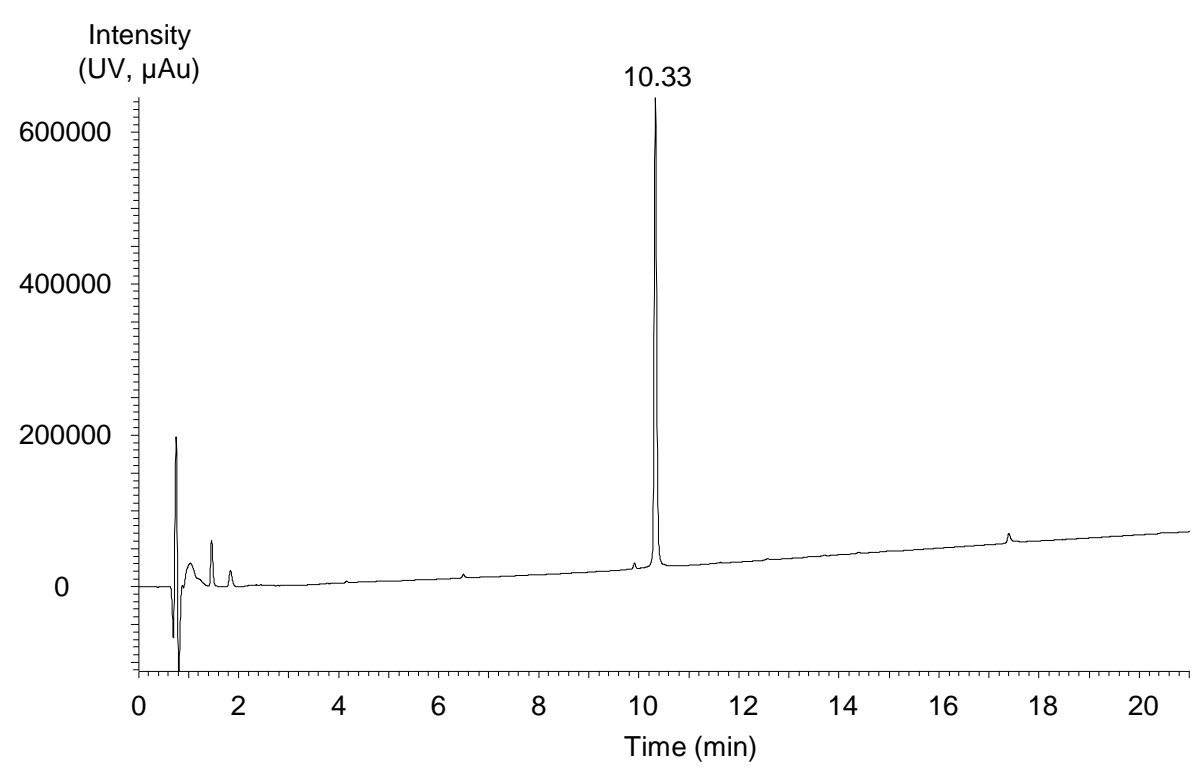

b)

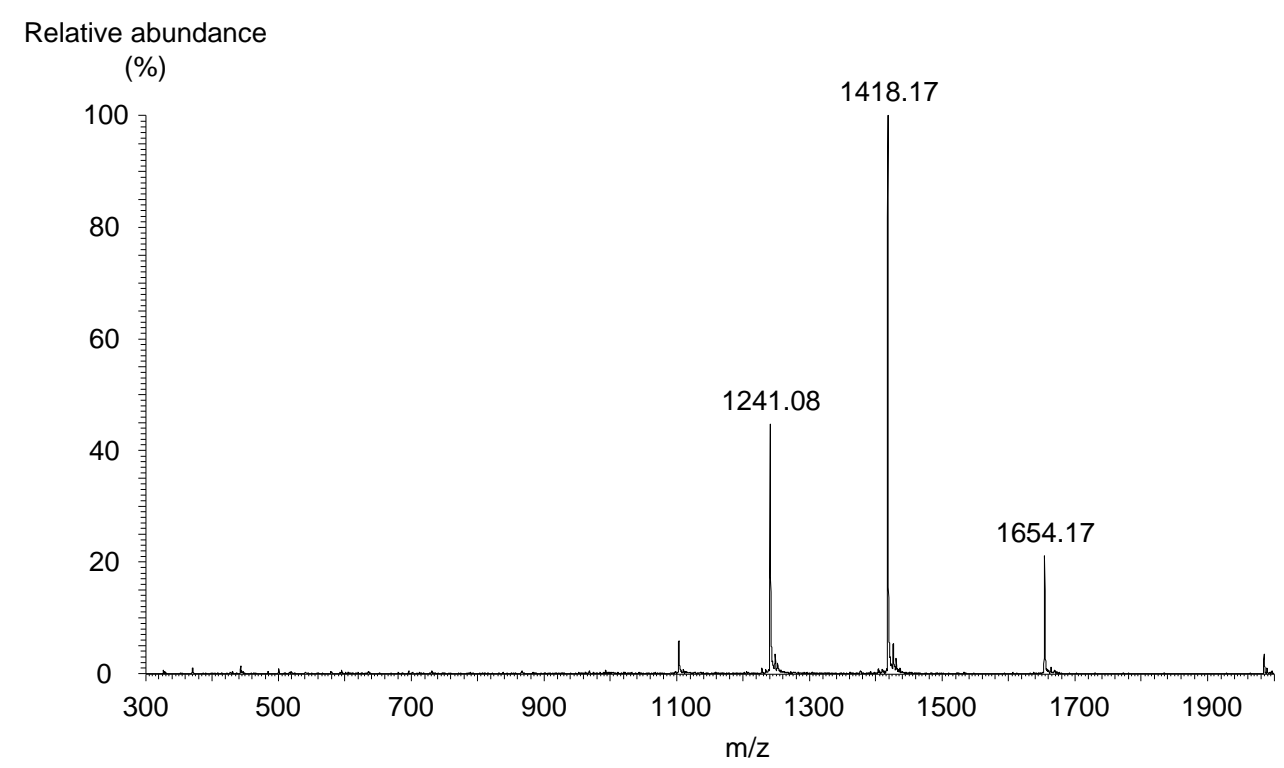

Figure S 134. LC-MS analysis of folded cK1-1f. a) LC trace. Eluent A $0.10 \%$ TFA in water, eluent B $0.10 \%$ TFA in $\mathrm{CH}_{3} \mathrm{CN}$, ACQUITY UPLC Peptide BEH $300 \AA 1.7 \mu \mathrm{m}(2.1 \times 150 \mathrm{~mm})$ column, $50{ }^{\circ} \mathrm{C}$, gradient $0-70 \% \mathrm{~B}$ in $20 \mathrm{~min}, 0.4 \mathrm{~mL} / \mathrm{min}$, UV detection. b) MS trace. $\mathrm{m} / \mathrm{z}=$ $1654.17\left([\mathrm{M}+6 \mathrm{H}]^{6+}\right), 1418.17\left([\mathrm{M}+7 \mathrm{H}]^{7+}\right), 1241.08\left([\mathrm{M}+8 \mathrm{H}]^{8+}\right)$, calcd for $\mathrm{M}$ (average): 9919.13, found: 9919.95 . 
a)

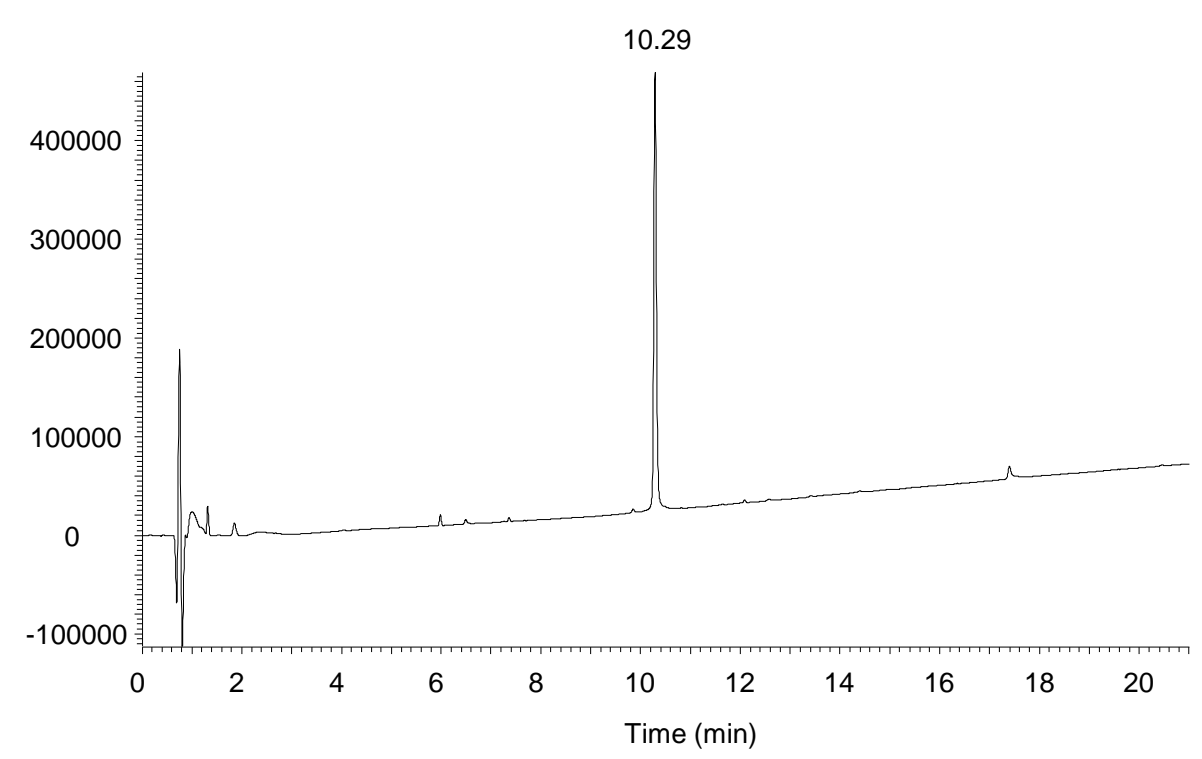

b)

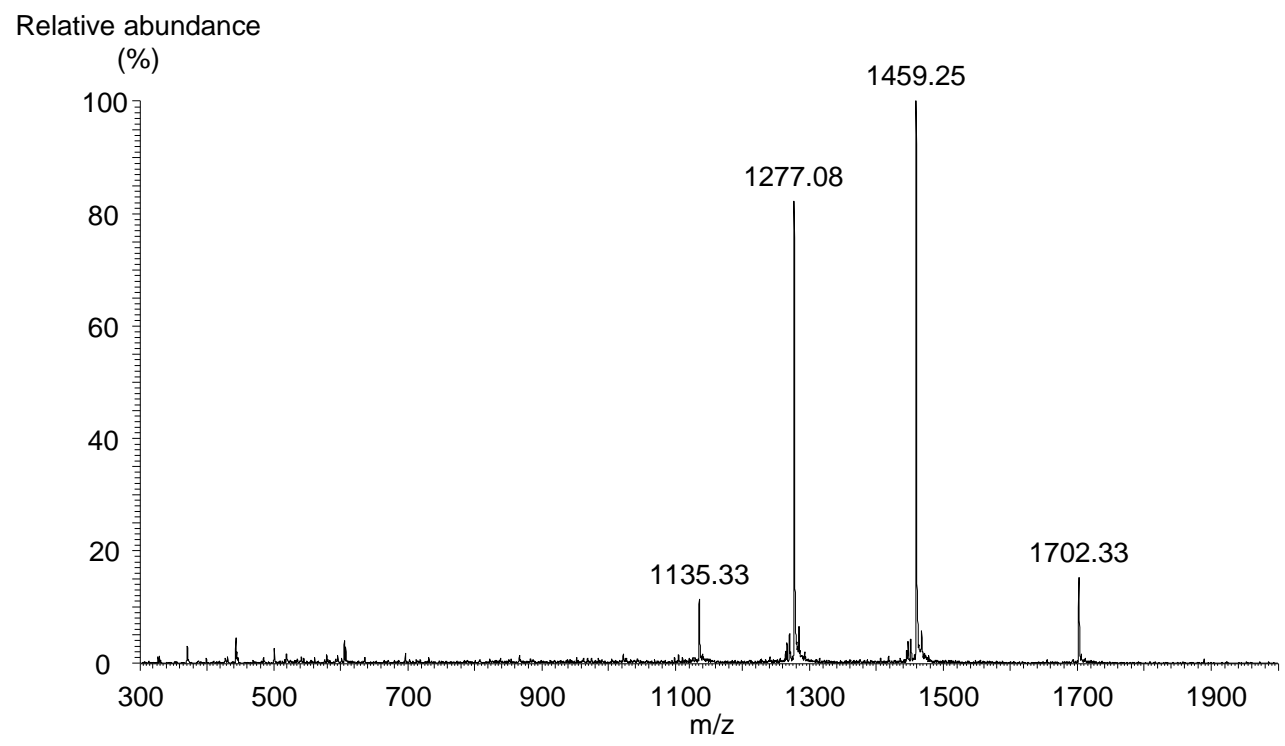

Figure S 135. LC-MS analysis of folded cK1-2f. a) LC trace. Eluent A $0.10 \%$ TFA in water, eluent B $0.10 \%$ TFA in $\mathrm{CH}_{3} \mathrm{CN}$, ACQUITY UPLC Peptide BEH $300 \AA 1.7 \mu \mathrm{m}(2.1 \times 150 \mathrm{~mm})$ column, $50{ }^{\circ} \mathrm{C}$, gradient $0-70 \% \mathrm{~B}$ in $20 \mathrm{~min}, 0.4 \mathrm{~mL} / \mathrm{min}$, UV detection. b) MS trace: $\mathrm{m} / \mathrm{z}=$ $1702.33\left([\mathrm{M}+6 \mathrm{H}]^{6+}\right), 1459.25\left([\mathrm{M}+7 \mathrm{H}]^{7+}\right), 1277.08\left([\mathrm{M}+8 \mathrm{H}]^{8+}\right), 1135.33\left([\mathrm{M}+9 \mathrm{H}]^{9+}\right)$; calcd for M (average): 10207.39, found 10208.34. 


\subsubsection{Disulfide bridge pattern of cK1-1f and cK1-2f}

The characterization of disulfide bridge patterns of cyclic derivatives cK1-1f and cK1-2f is given in Figure S 136. These results show that the disulfide bridge pattern of K1f formed during the folding process is not altered by the biotinylated linkers introduced to cyclize K1 domain.

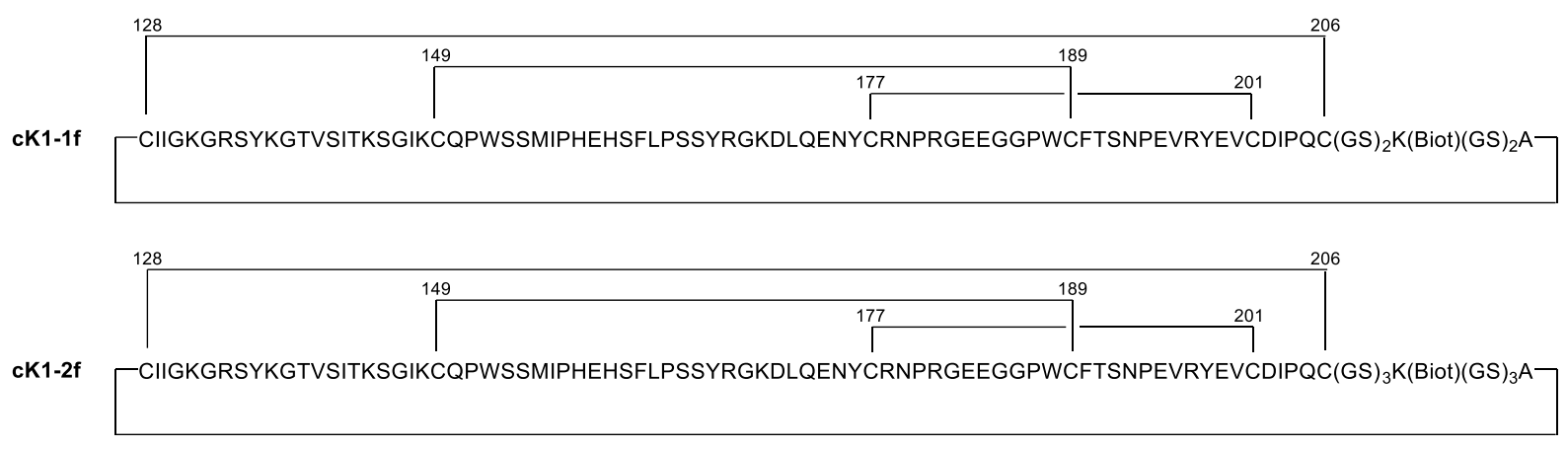

Figure S 136. Disulfide bridge patterns of K1f and its cyclic derivatives cK1-1f and cK1-2f.

Experimental determination of disulfide bridge patterns was achieved by enzymatic digestion of cK1-1f and cK1-2f domains and the resulting fragments were identified by mass spectrometry using non reducing conditions. Fragments obtained by digestion with trypsin permitted the direct assignment of Cys149-Cys189 disulfide bond whereas further digestion with the endoproteinase Asp-N was required to establish the formation of Cys128-Cys206 and Cys177-Cys201 disulfide bonds.

\section{Trypsin digestion}

Prior to use, cK1-1f and cK1-2f were purified by HPLC (eluent A 0.10\% TFA in water, eluent B $0.10 \%$ TFA in $\mathrm{CH}_{3} \mathrm{CN} /$ water: $4 / 1$ by vol., C18 XBridge $\mathrm{BEH} 300 \AA 3.5 \mu \mathrm{m} 2.1 \times 150 \mathrm{~mm}$ column, $50{ }^{\circ} \mathrm{C}$, gradient $0-10 \% \mathrm{~B}$ in $5 \mathrm{~min}$ then $10-80 \%$ in $5 \mathrm{~min}, 0.4 \mathrm{~mL} / \mathrm{min}$, detection at 250 $\mathrm{nm})$.

For each protein, the HPLC fractions containing the folded structure were collected and dried under vacuum and the residue was then dissolved in a solution of trypsin $(5 \mu \mathrm{L}, 50 \mathrm{ng} / \mu \mathrm{L})$ in $20 \mathrm{mM} \mathrm{NH}_{4} \mathrm{HCO}_{3}$. The digestion was carried out at $37{ }^{\circ} \mathrm{C}$ and the progress of the enzymatic reaction was monitored by MALDI-TOF mass spectrometry. Two main fragments were detected by HPLC after $5 \mathrm{~h}$ reaction (

Figure $S$ 137). The analysis of these fragments by mass spectrometry permitted the assignment of Cys ${ }^{149}$-Cys ${ }^{189}$ disulfide bond in both $\mathbf{c K 1}$ proteins (Figure S 138). 
a)

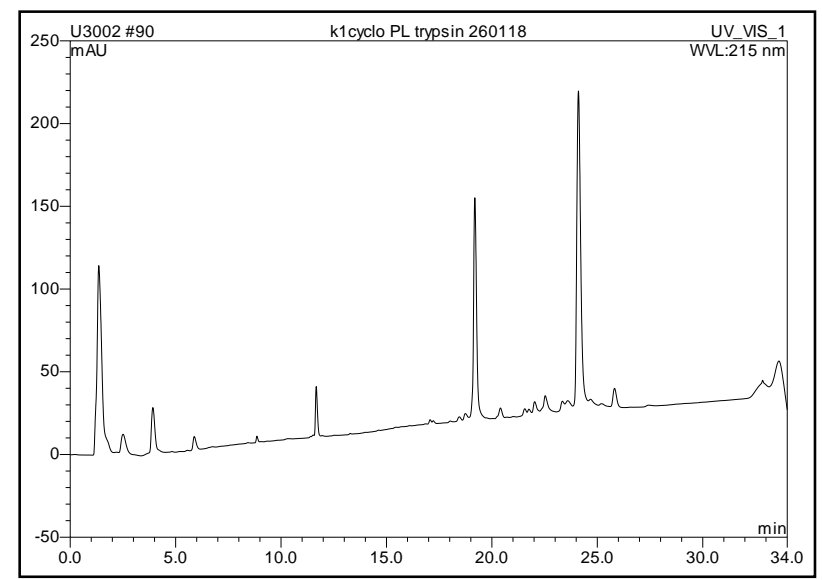

b)

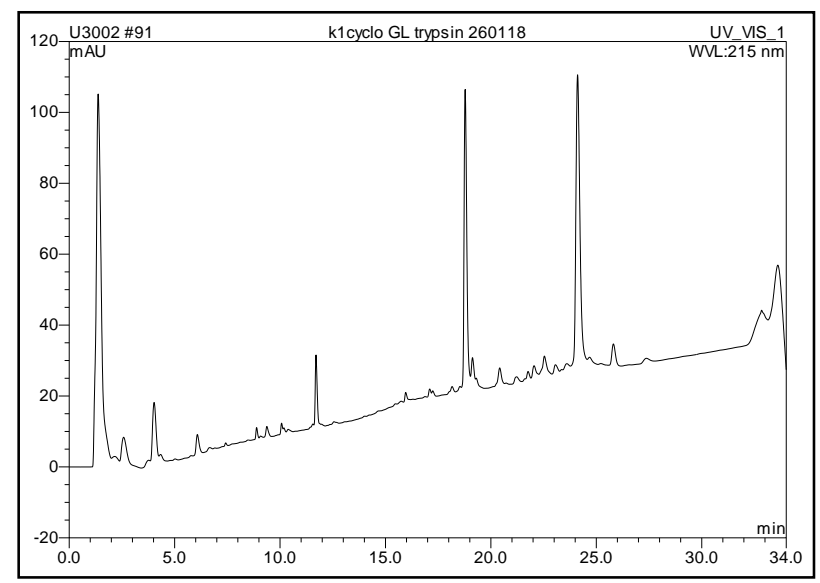

Figure S 137. HPLC analysis of the trypsic digest. Chromatogram obtained for cK1-1f (a) and cK1-2f (b). HPLC conditions: eluent A $0.10 \%$ TFA in water, eluent B $0.10 \%$ TFA in $\mathrm{CH}_{3} \mathrm{CN} /$ water: $4 / 1$ by vol., C18 XBridge BEH $300 \AA 3.5 \mu \mathrm{m} 2.1 \times 150 \mathrm{~mm}$ column, $50{ }^{\circ} \mathrm{C}$, gradient $0-40 \% \mathrm{~B}$ in $40 \mathrm{~min}, 0.4 \mathrm{~mL} / \mathrm{min}$, detection at $250 \mathrm{~nm}$. 


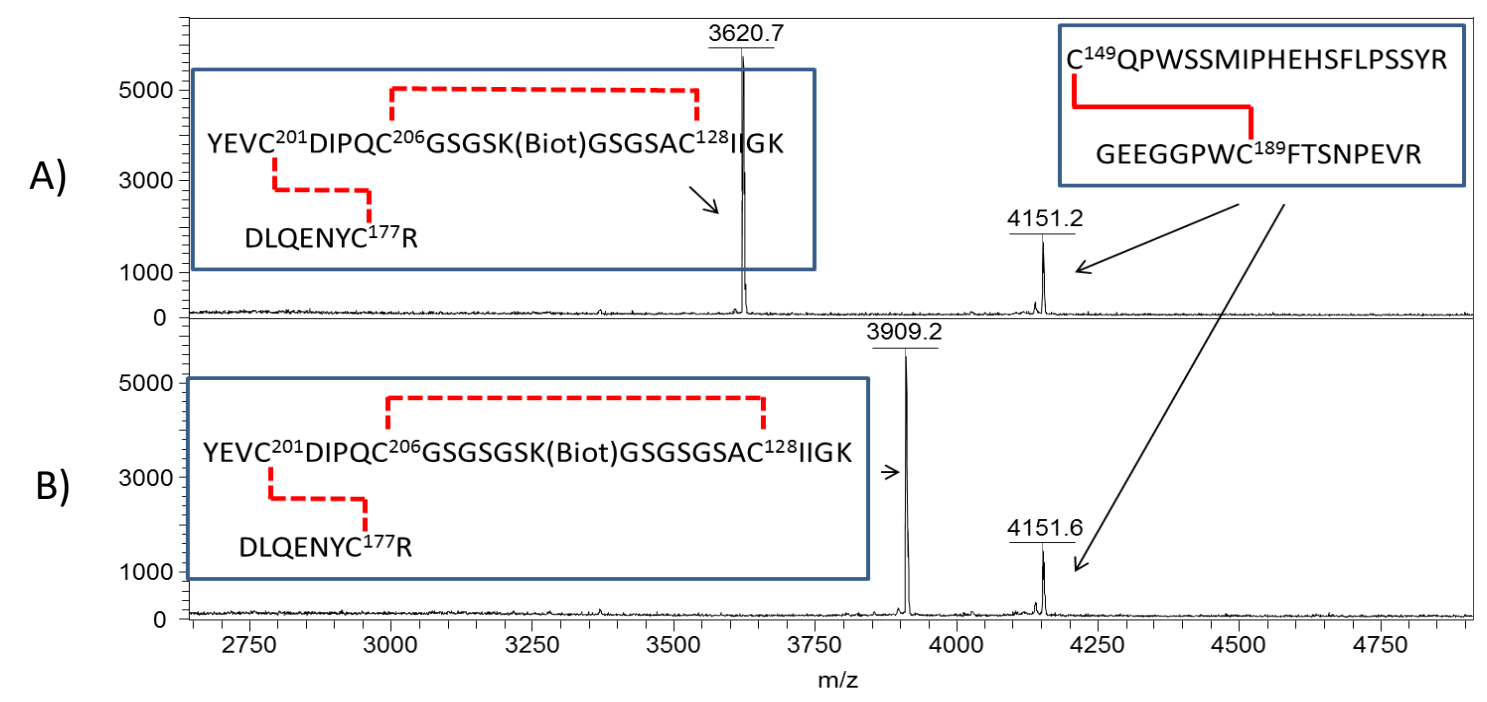

Figure S 138. MALDI-TOF analysis of the trypsic digest: results obtained with cK1-1f (A) and cK1-2f (B). Matrix 2,5-dihydroxybenzoic acid (DHB), positive detection mode.

\section{Asp- $N$ digestion}

Each trypsic digest was diluted with $0.1 \%$ TFA in water and the resulting mixture was purified by HPLC in order to isolate the fragment containing in its structure cysteines 128, 177, 201 and 206. The HPLC fractions in which this fragment was detected were concentrated and dried under vacuum and the obtained residue was then dissolved in a solution of endoproteinase $\mathrm{N}$ Asp $(3 \mu \mathrm{L}, 0.01 \mathrm{mg} / \mathrm{mL})$ in $20 \mathrm{mM} \mathrm{NH}_{4} \mathrm{HCO}_{3}$. The digestion was carried out at $37{ }^{\circ} \mathrm{C}$ and was run for $2 \mathrm{~min}$. The analysis of the new fragments by mass spectrometry permitted the assignment of $\mathrm{Cys}^{126}{ }_{-} \mathrm{Cys}^{206}$ and $\mathrm{Cys}^{177}{ }^{17 \mathrm{Cys}^{201}}$ disulfide bonds for both $\mathbf{c K 1}$ proteins (Figure S 139).

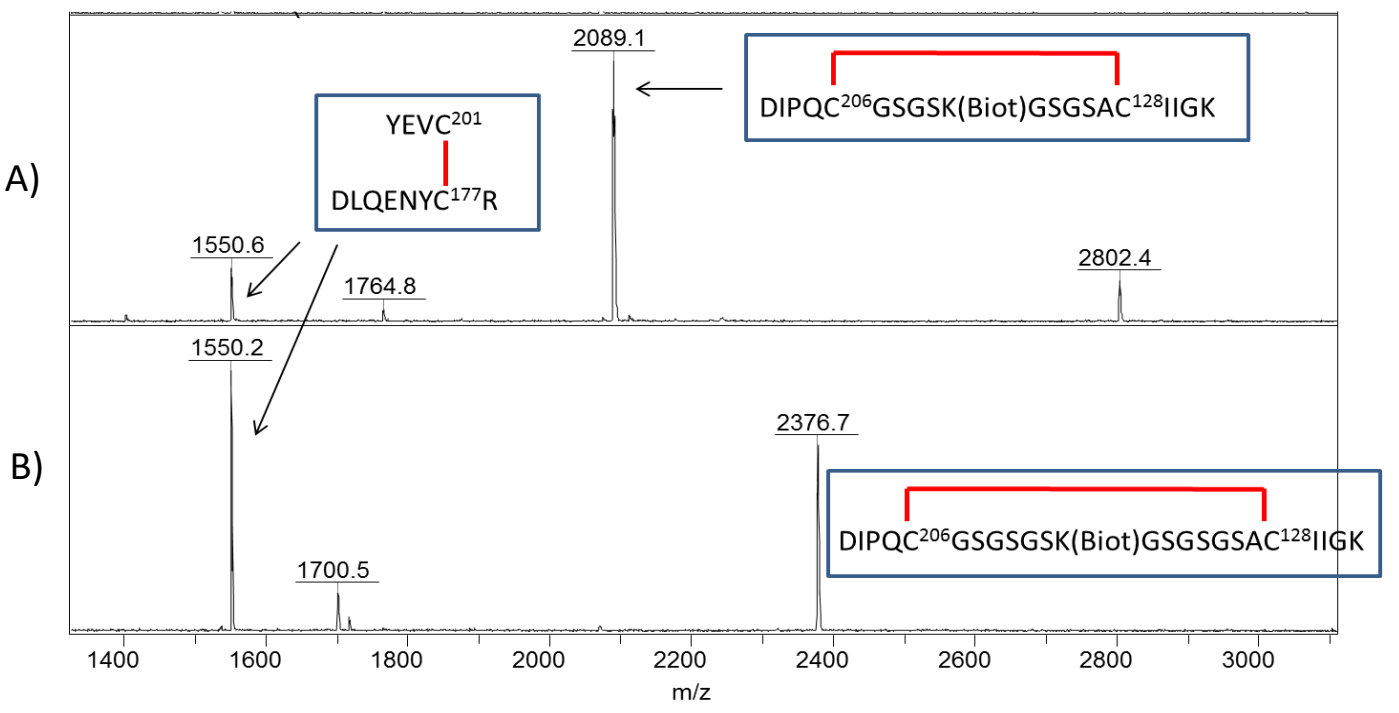

Figure $\mathbf{S}$ 139. MALDI-TOF analysis of the endoproteinase N-Asp digest: results obtained with cK1-1f (A) and cK1-2f (B). Matrix 2,5-dihydroxybenzoic acid (DHB), positive detection mode. 


\subsection{Alphascreen Assay (Figure 6d)}

Competition assays for binding of K1B, cK1-1f or cK1-2f to recombinant MET-Fc protein in competition with increasing concentrations of NK1 were performed in 384-well microtiter plates (OptiPlateTM-384, PerkinElmer@, CA, USA, $40 \mu \mathrm{L}$ of final reaction volume). Final concentrations were $20 \mathrm{nM}$ for K1B, cK1-1f or cK1-2f, $2 \mathrm{nM}$ for MET-Fc, 0-300 nM for NK1, $10 \mu \mathrm{g} \mathrm{mL}-1$ for streptavidin coated donor beads and protein A-conjugated acceptor beads. The buffer used for preparing all protein solutions and the bead suspensions was: PBS, $5 \mathrm{mM}$ HEPES pH 7.4, 0.1\% BSA.

K1B, cK1-1f or cK1-2f ( $5 \mu \mathrm{L}, 20 \mathrm{nM})$ was mixed with a solution of hMET-Fc $(5 \mu \mathrm{L}, 2 \mathrm{nM})$ and with solutions of NK1 $(10 \mu \mathrm{L}, 0-300 \mathrm{nM})$. The mixture was incubated for at $23{ }^{\circ} \mathrm{C} 60 \mathrm{~min}$ (final volume $20 \mu \mathrm{L}$ ). Protein A-conjugated acceptor beads $(10 \mu \mathrm{L}, 50 \mu \mathrm{g} \mathrm{mL}-1)$ were then added to the vials. The plate was incubated at $23{ }^{\circ} \mathrm{C}$ for $30 \mathrm{~min}$ in a dark box. Finally, streptavidin coated donor beads $(10 \mu \mathrm{L}, 50 \mu \mathrm{g} \mathrm{mL}-1)$ were added and the plate was further incubated at $23{ }^{\circ} \mathrm{C}$ for $30 \mathrm{~min}$ in a dark box. The emitted signal intensity was measured using standard Alpha settings on an EnSpire ${ }^{\circledR}$ Multimode Plate Reader (PerkinElmer).

\subsection{Biological assays (Figure 6e,f)}

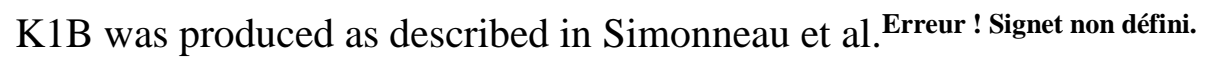

\subsubsection{Cell-based assay for MET signaling induced by cK1-1f and cK1-2f}

The assay was performed according to Simonneau et al. Erreur! Signet non défini. HeLa cells were t reated for 10 min with 300 pM mature HGF/SF (HGF), or with $10 \mathrm{nM} / 100 \mathrm{nM} \mathrm{K1/S}$, cK11f/S and cK1-2f/S.

Cell lysates were then analyzed by specific total MET and ERK or phospho-MET, phosphoAkt and phospho-ERK Western blot. Cells were collected by scraping and then lysed on ice with a lysis buffer (20 mM HEPES pH 7.4, $142 \mathrm{mM} \mathrm{KCl,} 5 \mathrm{mM} \mathrm{MgCl} 2,1 \mathrm{mM}$ EDTA, 5\% glycerol, $1 \%$ NP40 and $0.1 \%$ SDS) supplemented with freshly added protease and phosphatase inhibitors (\#P8340 and \#P5726, respectively, Sigma). Lysates were clarified by centrifugation $(20,000 \times \mathrm{g}, 15 \mathrm{~min})$ and protein concentration was determined (BCA protein assay Kit, Pierce®, Thermo scientific, IL, USA). The same protein amount of cell extracts was separated by either classical SDS-PAGE or NuPAGE (4-12\% or 10\% Bis-Tris precast gels) (Life technologies) and electrotransferred to polyvinylidene difluoride (PVDF) membranes (Merck Millipore). Membranes were probed with indicated primary antibodies, followed by incubation with appropriate HRP conjugated secondary antibodies. Protein-antibody complexes were visualized by chemiluminescence with the SuperSignal ${ }^{\circledR}$ West Dura Extended Duration Substrate (Thermo scientific), using a LAS-3000 imaging system (Fujifilm, Tokyo, Japan) or X-ray films (CL-Xposure ${ }^{\mathrm{TM}}$ Film, Thermo scientific). 


\subsubsection{Scattering Assay}

The assay was performed according to Simonneau et al. ${ }^{10}$

Capan cells were seeded at low density (2,000 cells/well on a 12-well plate) to form compact colonies. After treatment, when colony dispersion was observed, the cells were fixed and colored by Hemacolor ${ }^{\circledR}$ stain (Merck, Darmstadt, Germany) according to the manufacturer's instructions. Representative images were captured using a phase contrast microscope with 40x magnification (Nikon Eclipse TS100, Tokyo, Japan). 


\section{References}

1. Abbas M., Bethke J. \& Wessjohann L. A. One pot synthesis of selenocysteine containing peptoid libraries by Ugi multicomponent reactions in water. Chem. Commun., 541-543 (2006).

2. Miura C., Kiyama M., Iwano S., Ito K., Obata R., Hirano T. et al. Synthesis and luminescence properties of biphenyl-type firefly luciferin analogs with a new, nearinfrared light-emitting bioluminophore. Tetrahedron 69, 9726-9734 (2013).

3. Boll E., Drobecq H., Ollivier N., Blanpain A., Raibaut L., Desmet R. et al. One-pot chemical synthesis of small ubiquitin-like modifier (SUMO) protein-peptide conjugates using bis(2-sulfanylethyl)amido peptide latent thioester surrogates $\mathrm{Nat}$. Protoc. 10, 269-292 (2015).

4. Ollivier N., Raibaut L., Blanpain A., Desmet R., Dheur J., Mhidia R. et al. Tidbits for the synthesis of bis(2-sulfanylethyl)amido (SEA) polystyrene resin, SEA peptides and peptide thioesters. J. Pept. Sci. 20, 92-97 (2014).

5. Ollivier N., Dheur J., Mhidia R., Blanpain A. \& Melnyk O. Bis(2-sulfanylethyl)amino native peptide ligation. Org. Lett. 12, 5238-5241 (2010).

6. Ollivier N., Desmet R., Drobecq H., Blanpain A., Boll E., Leclercq B. et al. A simple and traceless solid phase method simplifies the assembly of large peptides and the access to challenging proteins. Chem. Sci. 8, 5362-5370 (2017).

7. $\quad$ Fang G.-M., Li Y.-M., Shen F., Huang Y.-C., Li J.-B., Lin Y. et al. Protein chemical synthesis by ligation of peptide hydrazides. Angew. Chem. Int. Ed. 50, 7645-7649 (2011).

8. Ruff Y., Garavini V. \& Giuseppone N. Reversible native chemical ligation: a facile access to dynamic covalent peptides. J. Am. Chem. Soc. 136, 6333-6339 (2014).

9. Ollivier N., Vicogne J., Vallin A., Drobecq H., Desmet R., El-Mahdi O. et al. A onepot three-segment ligation strategy for protein chemical synthesis. Angew. Chem. Int. Ed. 51, 209-213 (2012).

10. Simonneau C., Berenice L., Mougel A., Adriaenssens E., Paquet C., Raibaut L. et al. Semi-synthesis of a HGF/SF kringle one (K1) domain scaffold generates a potent in vivo MET receptor agonist. Chem. Sci. 6, 2110-2121 (2015). 
